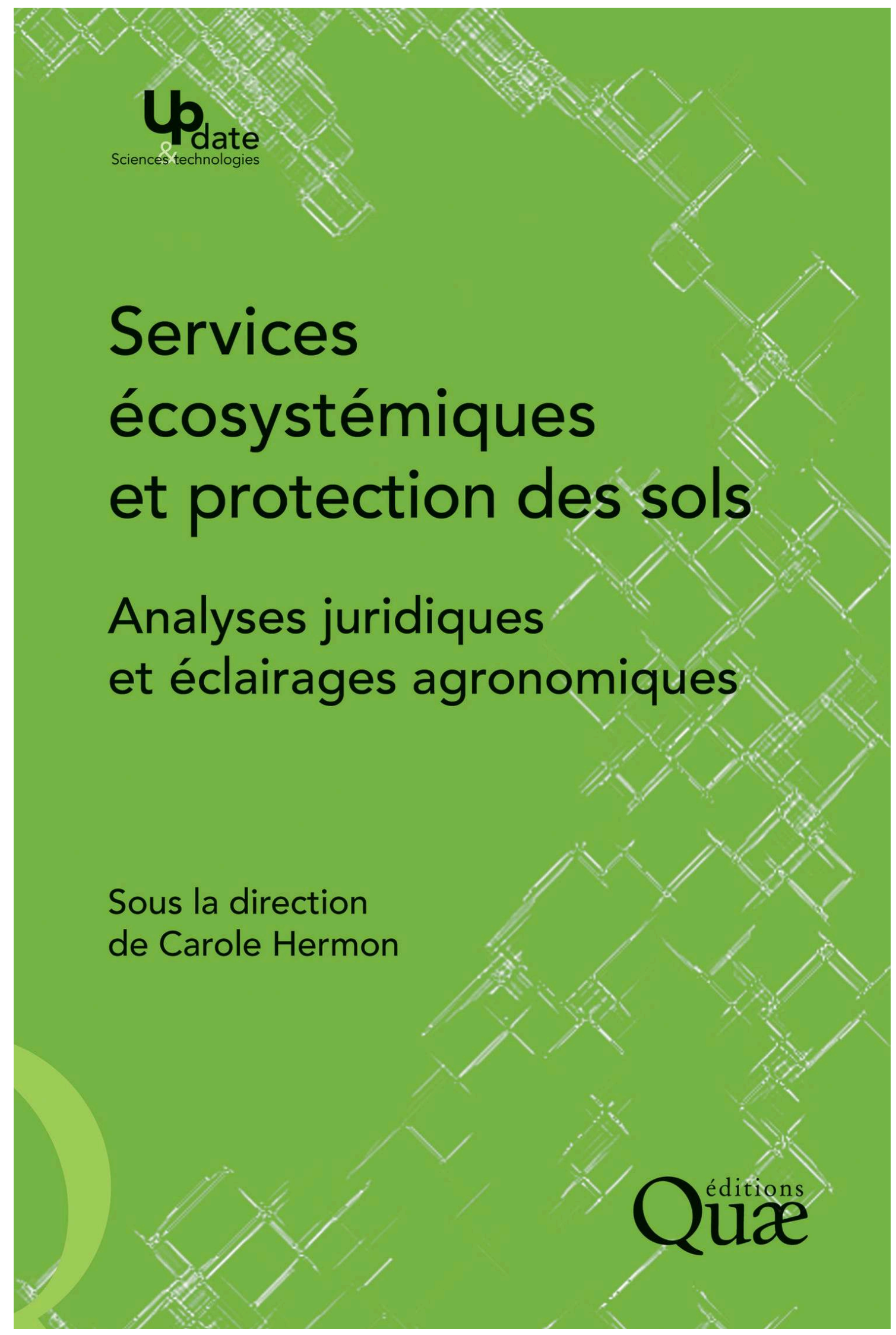




\section{Services écosystémiques et protection des sols}

Analyses juridiques et éclairages agronomiques

\section{Carole Hermon (dir.)}

Éditeur : Éditions Quæ

Année d'édition : 2018

Date de mise en ligne : 17 mars 2021

Collection : Update Sciences \& Technologie

\section{o orentitition ebooks}

http://books.openedition.org

Édition imprimée

Nombre de pages : 326

Référence électronique

HERMON, Carole (dir.). Services écosystémiques et protection des sols : Analyses juridiques et éclairages agronomiques. Nouvelle édition [en ligne]. Versailles : Éditions Quæ, 2018 (généré le 17 mars 2021).

Disponible sur Internet : <http://books.openedition.org/quae/30685>.

(c) Éditions Quæ, 2018

Creative Commons - Attribution - Pas d'Utilisation Commerciale - Pas de Modification 4.0 International - CC BY-NC-ND 4.0 


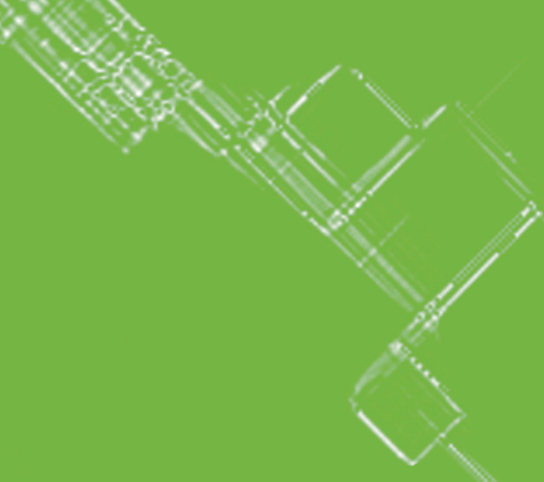

Services

écosystémiques

et protection des sols

Analyses juridiques et éclairages agronomiques

Sous la direction de Carole Hermon
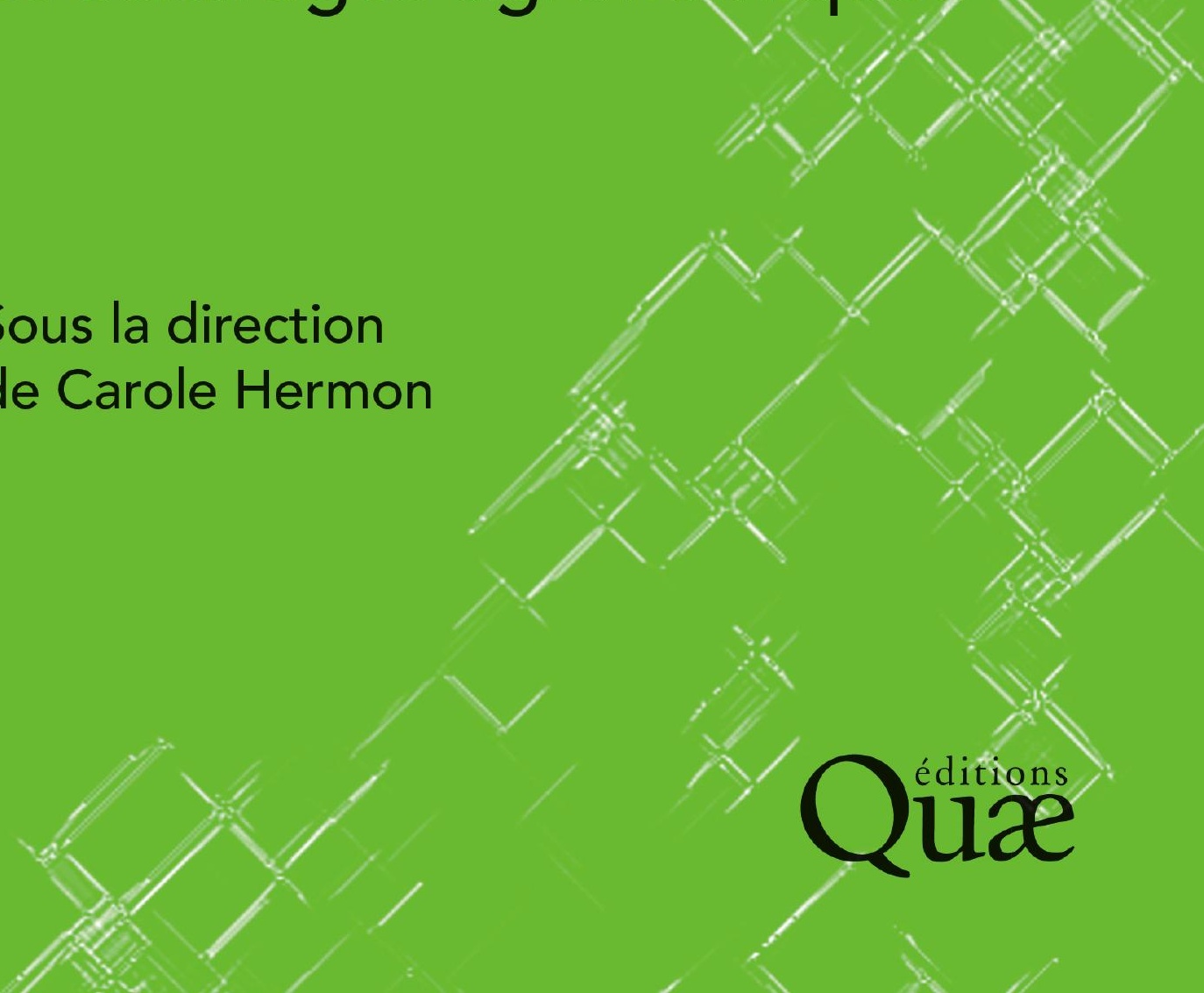


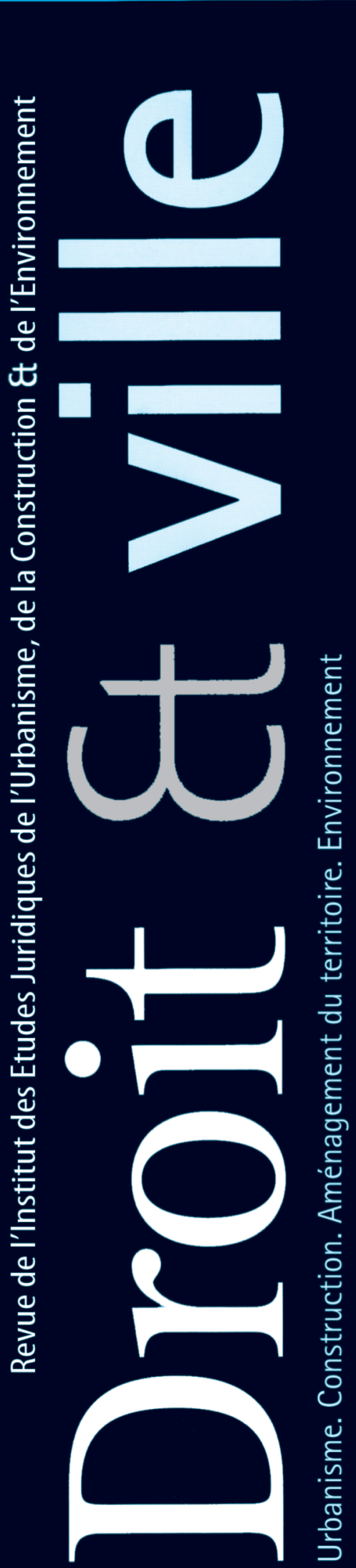

- Services écosystémiques et protection des sols. Analyses juridiques et eclairages agronomiques

\section{Avant-propos $;$ par Carole HERMON}

\section{PROPOS INTRODUCTIFS}

Protection des sols et services écosystémiques:

La nécessité dune reconsideration epistémologique »

par Liliane ICHER et Bastien ALIDOR

La protection du sol en droit " par Garole HERMON

Le sol agricole, une ressource indispensable négligée »; par Ariane CHABERT et Jean-Pierre SARTHOU

Proprieté et protection des sols. Réflexions civilistes sur la prise en compte de la qualité des sols $;$ par Lionel BOSC

\section{PROPOSITIONS POUR UNE PROTECTION DES SOLS EN DROIT}

A. L'INTÉGRATION DE LA NOTION DE SERVICE

ÉCOSYSTEMIQUE EN DROT

« Brève histoire de l'intégration de la notion de service écosystémique en droit " par Isabelle DOUSSAN

"Les services écosystémiques", une notion fonctionnelle " par Mélodie FEVRE

«La qualification juridique des services ecosystémiques" par Guillaume BEAUSSONIE

B. LES SERVIGES ECOSYSTEMIQUES RENDUS PAR LES SOLS DU POINT DE VUE DE LAGRONOMIE

"Agriculture de conservation des sols

et services écosystémiques 》

par Ariane CHABERT et Jean-Pierre SARTHOU

C. LA NOTION DE SERVICE ÉCOSYSTÉMIQUE PEUT-ELLE

FONDER UN NOUVEAU RÉGIME DE PROTECTION?

1) Service écosystémique et financements publics

"La depense publique en matiere environnementale :

lexemple de la protection des sols » par Liliane ICHER

2) Service écosystémique et droit des contrats

Services écosystemiques et contrat - Quelle obligation contractuelle environnementale? " par Matthieu POUMAREDE «Compensation et services écosystemiques "

par Bastien ALIDOR

«La commande publique de compensation environnementale : un impensé de la loi Biodiversite s par flelène HOEPFFNER

Travail du sol, services écosystémiques, et bail rural »

par Didier KRAJESKI

3) Service écosystémique et droit de la responsabilité " L'incidence des services écosystémiques en droit de la responsabilité civile » par Séverin JEAN

\section{ANNEXES \\ Glossaire \\ Bibliographie sélective}


Fondateur de la Revue : Michel DESPAX

Directeur de publication : Matthieu POUMAREDE, Professeur à l'Université Toulouse 1 Capitole, Directeur de l'IEJUC

Rédactrice en chef: Florence BAYARD-JAMMES, Enseignant-chercheur à la Toulouse Business School, Université de Toulouse

\section{COMITÉ SCIENTIFIQUE}

Directeur scientifique : Daniel TOMASIN, Professeur émérite de l'Université Toulouse 1 Capitole Pierre DELVOLVE, Professeur émérite de l'Université Paris 2 Panthéon-Assas

Fernand BOUYSSOU, Agrégé de droit public, Avocat

Françoise FRAYSSE, Professeur à l'Université Toulouse 1 Capitole

Hugues KENFACK, Doyen de la Faculté de droit de Toulouse, Professeur à l'Université Toulouse 1 Capitole

Hugues PERINET-MARQUET, Professeur à l'Université Paris 2 Panthéon-Assas

François PRIET, Professeur à l'Université d'Orléans

Thierry REVET, Professeur à l'Université Paris 1 Panthéon-Sorbonne

Gabriel ROUJOU de BOUBEE, Professeur émérite de l'Université Toulouse 1 Capitole

Louis ROZES, Professeur émérite de l'Université Toulouse 1 Capitole

Corinne SAINT-ALARY-HOUIN, Professeur à l'Université Toulouse 1 Capitole

Jérôme TREMEAU, Professeur à l'Université Aix-Marseille III

\section{COMITÉ DE LECTURE}

Guillaume BEAUSSONIE, Professeur à l'Université Toulouse 1 Capitole Julien BÉTAILLE, Maître de conférences à l'Université Toulouse 1 Capitole Vincent DUSSART, Professeur à l'Université Toulouse 1 Capitole Carole HERMON, Professeure à l'Université Toulouse 1 Capitole Hélène HOEPFFNER, Professeur à l'Université Toulouse 1 Capitole Didier KRAJESKI, Professeur à l'Université Toulouse 1 Capitole Matthieu POUMARÈDE, Professeur à l'Université Toulouse 1 Capitole Moussan THIOYE, Maître de conférences à l'Université Toulouse 1 Capitole Séverin JEAN, Maître de conférences à l'Université Toulouse 1 Capitole François-Guy TRÉBULLE, Professeur à l'Université Paris 1 Panthéon-Sorbonne

\section{CORRESPONDANTS ÉTRANGERS}

ÉTATS-UNIS, Jane C. GINSBURG, Professeur à la Columbia University, New-York ESPAGNE, Antoni VAQUER, Professeur à l'Université de Lleida ITALIE, Alberto LUCARELLI, Professeur à l'Université « Frédérico II » de Naples JAPON, Hitoshi TERAO, Professeur à l'Université de Niigata SÉNÉGAL, Abdoullah CISSE, Recteur de l'Université sénégalaise de Bambey VIETNAM, Ngoc Dien NGUYEN, Professeur à l'Université nationale du Viet Nam à Ho-Chi-Minh Ville 


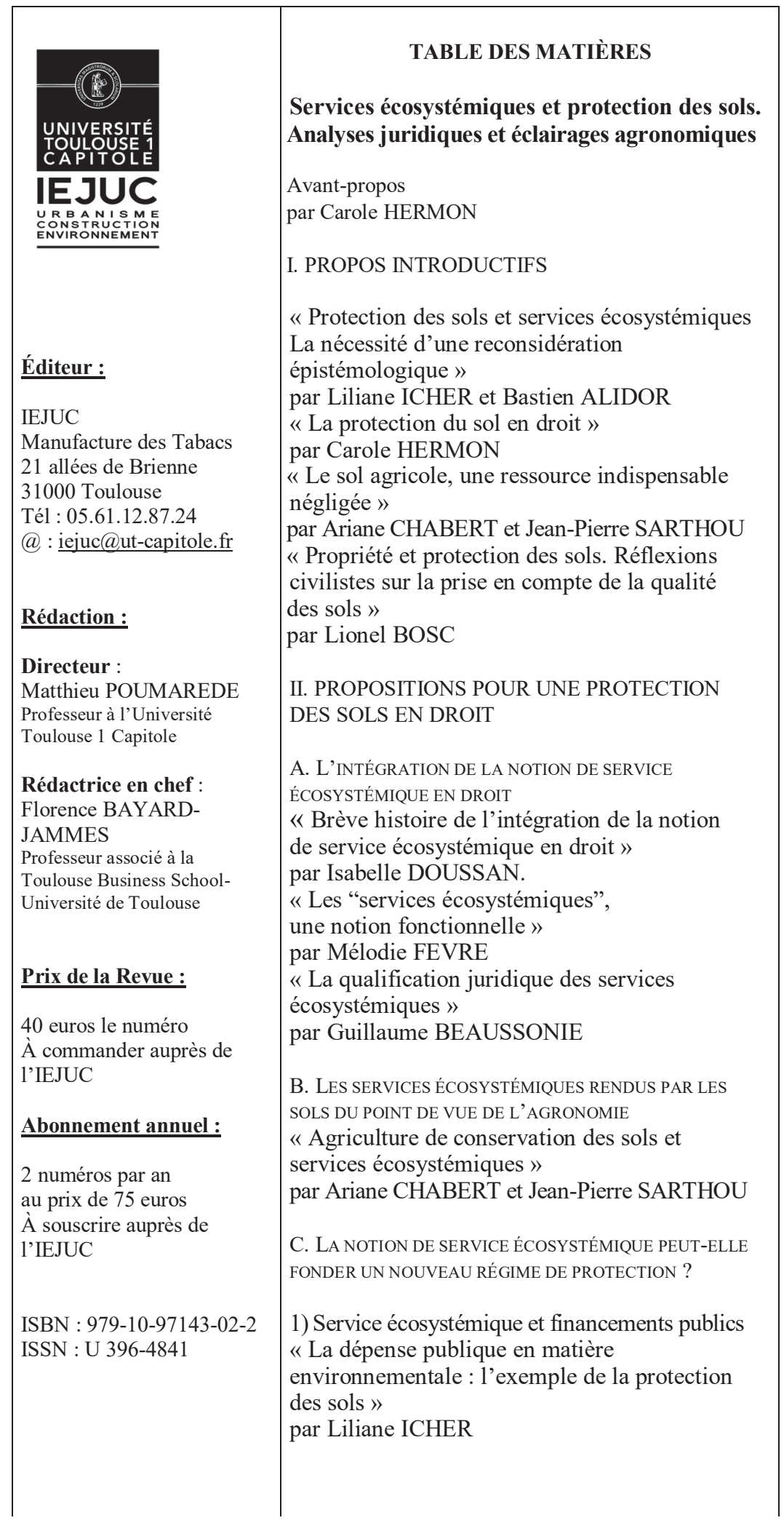




2) Service écosystémique et droit des contrats
"Services écosystémiques et contrat - Quelle
obligation contractuelle environnementale?»
par Matthieu POUMAREDE
"Compensation et services écosystémiques »
par Bastien ALIDOR
«La commande publique de compensation
environnementale : un impensé de la loi
Biodiversité »
par Hélène HOEPFFNER
« Travail du sol, services écosystémiques,
et bail rural »
par Didier KRAJESKI
3) Service écosystémique et droit de la
responsabilité
«L'incidence des services écosystémiques
en droit de la responsabilité civile»
par Séverin JEAN
ANNEXES
Glossaire
Bibliographie sélective




\section{« SERVICES ÉCOSYSTEMIQUES ET PROTECTION DES SOLS. ANALYSES JURIDIQUES ET ÉCLAIRAGES AGRONOMIQUES »}

La version ePub de l'ouvrage est publiée aux éditions Quæ (ISBN : 978-2-7592-2791-4) 



\section{SOMMAIRE}

\section{Services écosystémiques et protection des sols. Analyses juridiques et éclairages agronomiques}

« Avant-propos », Carole Hermon, Professeure, Université Toulouse 1 Capitole, Responsable scientifique, Idex ATS/T2SEC

\section{PROPOS INTRODUCTIFS}

Liliane Icher, docteure en droit, post-doctorante Idex T2SEC, et Bastien Alidor, doctorant, IEJUC, "Protection des sols et services écosystémiques. La nécessité d'une reconsidération épistémologique » Carole Hermon, Professeure, Université Toulouse Capitole, « La protection du sol en droit »

Ariane Chabert, docteure en agronomie, post-doctorante Idex T2SEC, et Jean-Pierre Sarthou, Professeur, INRA/INP-ENSAT, « Le sol agricole, une ressource indispensable négligée "

Lionel Bosc, doctorant, IEJUC, «Propriété et protection des sols. Réflexions civilistes sur la prise en compte de la qualité des sols »

\section{PROPOSITIONS POUR UNE PROTECTION DES SOLS EN DROIT}

\section{A. L'INTÉGRATION DE LA NOTION DE SERVICE ÉCOSYSTÉMIQUE EN DROIT}

Isabelle Doussan, Directrice de recherche INRA, Université Côte d'Azur, « Brève histoire de l'intégration de la notion de service écosystémique en droit »

Mélodie Fèvre, docteure en droit, Université Côte d'Azur, « Les « services écosystémiques », une notion fonctionnelle »

Guillaume Beaussonie, Professeur, Université Toulouse Capitole, «La qualification juridique des services écosystémiques »

B. LES SERVICES ÉCOSYSTÉMIQUES RENDUS PAR LES SOLS DU POINT DE VUE DE L'AGRONOMIE

Ariane Chabert, docteure en agronomie, post-doctorante Idex T2SEC, et Jean-Pierre Sarthou, Professeur, INRA/INP-ENSAT, "Agriculture de conservation des sols et services écosystémiques » 
C. LA NOTION DE SERVICE ÉCOSYSTÉMIQUE PEUT-ELLE FONDER UN NOUVEAU RÉGIME DE PROTECTION?

1) Service écosystémique et financements publics

Liliane Icher, docteure en droit, post-doctorante Idex T2SEC, « La dépense publique en matière environnementale : l'exemple de la protection des sols »

2) Service écosystémique et droit des contrats

Matthieu Poumarède, Professeur, Université Toulouse Capitole, «Services écosystémiques et contrat. Quelle obligation contractuelle environnementale?»

Bastien Alidor, doctorant, IEJUC, « Compensation et services écosystémiques »"

Hélène Hoepffner, Professeur, Université Toulouse Capitole, « La commande publique de compensation environnementale : un impensé de la loi Biodiversité »

Didier Krajeski, Professeur, Université Toulouse Capitole, « Travail du sol, services écosystémiques et bail rural»

3) Service écosystémique et droit de la responsabilité

Séverin Jean, Maître de conférences, Université Toulouse Capitole, «L'incidence des services écosystémiques en droit de la responsabilité civile»

\section{ANNEXES}

Glossaire

Bibliographie sélective 


\title{
Avant-propos
}

\author{
Carole HERMON \\ Professeure, Université Toulouse 1 Capitole \\ IEJUC (EA 1919) \\ Responsable scientifique, Idex ATS/T2SEC
}

Le présent ouvrage a été rédigé dans le cadre du programme de recherche T2SEC, Travail du sol, services écosystémiques et compensation. Aspects agronomiques et juridiques ${ }^{1}$. L'étude portant sur le sol, elle s'est voulue interdisciplinaire, croisant les analyses d'agronomes et de juristes ${ }^{2}$, avec une prévalence des réflexions juridiques. Cette dominante juridique entendait répondre à la faiblesse relative de la recherche en droit au regard des autres disciplines et à son manque de visibilité au sein de la communauté scientifique, tant sur la question du sol que sur celle des services écosystémiques.

Pour faciliter la construction d'une recherche collective, l'hypothèse de travail initiale a été étroitement circonscrite et formulée ainsi : certains systèmes de production agricole, exemple étant pris de l'agriculture de conservation, contribuent à maintenir ou restaurer les fonctions du sol et les services écosystémiques associés. L'intégration de ces services en droit pourrait contribuer à valoriser ces méthodes de production et, ce faisant, à donner aux sols une protection jusqu'alors inexistante. La réflexion a ainsi été centrée sur les fonctions et services écosystémiques, l'étude de la compensation

1. Idex, ATS 2015, « Systèmes de gestion durable des ressources naturelles et de l'environnement », Université Toulouse 1 Capitole, IEJUC (EA 1919).

2. L'équipe associe deux laboratoires (l'IEJUC, EA 1919, Université Toulouse 1 Capitole et l'UMR INRA/INP-ENSAT 1248 AGIR), et treize chercheurs et enseignants-chercheurs en droit et en agronomie. $C f$. infra Liste des auteurs. 
écologique n'intervenant que comme une voie potentielle de leur reconnaissance en droit.

À ce titre, les agronomes ont procédé à une étude bibliographique complétée par des études de terrain et, à partir de ces matériaux, dressé un inventaire des services écosystémiques du sol préservés ou confortés par l'agriculture de conservation. Les juristes, confrontés à un état de la science moins avancé, ont élargi la perspective en commençant par identifier les normes relatives à la protection des sols, repéré la notion de service écosystémique en droit et proposé une qualification de ces services. Ils ont ensuite analysé certains des instruments qui peuvent intégrer la notion de service et/ou les pratiques de l'agriculture de conservation : le contrat, y compris le bail rural et les contrats liés à l'obligation de compensation, la responsabilité civile, les aides publiques et la comptabilité publique. D'autres techniques n'ont pu être étudiées, particulièrement celles en lien avec la lutte contre le changement climatique. Le présent ouvrage ne prétend donc nullement à l'exhaustivité.

S'agissant d'un travail collectif et interdisciplinaire qui avait l'ambition de conduire à une réflexion commune, un premier temps a été consacré à la mise en commun des savoirs et à l'acquisition d'une culture partagée. Pour ce faire, un glossaire a été établi, présenté en annexe, qui avait pour seul objet de faciliter le dialogue interdisciplinaire, et des ateliers ont été organisés avec des chercheurs et enseignants-chercheurs invités ${ }^{3}$. Le travail de recherche et d'écriture individuel engagé dans un deuxième temps a été partagé dans un troisième temps et confronté aux critiques, analyses et propositions de l'équipe. Il en ressort cette étude collective, interdisciplinaire, où les contributions de chacun ont été enrichies pour former un ensemble que nous espérons cohérent et susceptible de susciter d'autres recherches sur un thème qui est loin d'être épuisé.

3. Quatre ateliers ont été organisés entre mai 2016 et mars 2017 sur : la notion de services écosystémiques, la nature juridique des services écosystémiques, la compensation écologique, les biens communs. Treize chercheurs, enseignants-chercheurs, experts ont été entendus dans ce cadre ( $C f$. infra Remerciements). Que chacun soit ici une nouvelle fois remercié de sa contribution à notre réflexion collective. 


\section{Liste des auteurs}

- B. Alidor, doctorant, IEJUC, Université Toulouse Capitole

- G. Beaussonie, Professeur, IEJUC, Université Toulouse Capitole

- L. Bosc, doctorant, IEJUC, Université Toulouse Capitole

- A. Chabert, post-doctorante, IEJUC, Université Toulouse Capitole, INRA/INP-ENSAT

- I. Doussan, Directrice de recherche, INRA, GREDEG UMR CNRS 7321, Université Côte d'Azur

- M. Fèvre, docteure en droit, GREDEG UMR CNRS 7321, Université Côte d'Azur

- C. Hermon, Professeure, IEJUC, Université Toulouse Capitole, responsable scientifique

- H. Hoepffner, Professeur, IEJUC, Université Toulouse Capitole

- L. Icher, post-doctorante, IEJUC, Université Toulouse Capitole

- S. Jean, Maître de conférences, IEJUC, Université Toulouse Capitole

- D. Krajeski, Professeur, IEJUC, Université Toulouse Capitole

- M. Poumarède, Professeur, IEJUC, Université Toulouse Capitole

- J.-P. Sarthou, Professeur, INRA/INP-ENSAT. 


\section{Remerciements}

Cet ouvrage est aussi le fruit des échanges avec plusieurs chercheurs et experts qui sont venus nous apporter leur point de vue sur nos axes de réflexions. Nous les remercions de leur contribution.

- J. Delord, Philosophe

- V. Devictor, Chargé de recherche, CNRS, Université Montpellier 3

- T. Dutoit, Directeur de recherche, CNRS, IUT Avignon

- E. Fatome, Professeur émérite, Université Paris 1 Panthéon Sorbonne

- G. Froger, Professeure, Université Toulouse Jean Jaurès

- J. Genin, Directeur de l'investissement et des projets, CdC Biodiversité, chef de l'Agence Sud-Ouest

- C. Ianello, Professeur, Seconda Università du Napoli

- A. Langlais, Chargée de recherche, CNRS, Université Rennes

- M. Lucas, Maître de conférences, IUT Avignon

- G.-J. Martin, Professeur émérite, Université Côte d'Azur

- P. Méral, Directeur de recherche, IRD, Université Montpellier 3

- T. Revet, Professeur, Université Paris 1 Panthéon Sorbonne

- O. Thérond, Chargé de recherche, INRA, Université de Lorraine

- S. Vanuxem, Maître de conférences, Université Côte d'Azur 


\section{PROPOS INTRODUCTIFS}





\title{
Protection des sols et services écosystémiques. La nécessité d'une reconsidération épistémologique
}

\author{
Liliane ICHER \\ Docteure en droit \\ post-doctorante Idex T2SEC
}

Bastien ALIDOR

Doctorant, IEJUC

Une réflexion épistémologique semble être un préalable indispensable à toute étude académique mais elle a paru encore plus nécessaire à l'écriture du présent ouvrage.

D'abord, parce qu'il s'agit d'un ouvrage collectif et ensuite parce que la recherche s'est voulue interdisciplinaire ${ }^{1}$. Tout en conservant sa liberté de pensée, chaque auteur a souhaité partager certaines notions et parvenir, par ajustements successifs, à construire un discours commun pour éviter que les chapitres ne souffrent d'irrémédiables contradictions. Le but de l'équipe est d'élaborer une démonstration cohérente, et non d'accoler des raisonnements individuels les uns aux autres. L'épistémologie est devenue alors une opportunité pour tous de se (re)questionner sur son champ de compétence pour savoir ce qu'il est nécessaire de clarifier pour les co-auteurs et les lecteurs non spécialistes.

\footnotetext{
1. Si la perspective pluridisciplinaire conduit à une juxtaposition des disciplines et la transdisciplinarité vise à faire disparaître les frontières disciplinaires et à générer la constitution d'un savoir autonome, l'approche interdisciplinaire consiste en une « articulation de savoirs qui entraîne, par approches successives, comme dans un dialogue, des réorganisations partielles des champs théoriques en présence », F. Ost et M. van de Kerchove, 1987, Jalons pour une théorie critique du droit, Bruxelles, Pub. des Facultés universitaires de SaintLouis.
} 
Ce choix de l'interdisciplinarité et le désir de décloisonner les processus de connaissances nous ont ainsi éloigné de la conception classique de la science.

La conception de la science la plus largement admise, ou du moins celle qui a le plus profondément marqué notre imaginaire collectif, correspond à une vision cartésienne de la connaissance tendant à assimiler science et vérité. Parmi les préconisations méthodologiques de Descartes, deux ont été particulièrement suivies et ont durablement façonné la conduite des recherches académiques. L'auteur conseille de diviser les domaines de connaissance et les problématiques pour mieux les étudier puis d'utiliser la méthode de l'entonnoir en partant du problème le plus simple pour aller vers des questions de plus en plus précises ${ }^{2}$. Cette spécialisation disciplinaire a par la suite été encouragée par Kant ${ }^{3}$, reprise par Comte ${ }^{4}$ et adaptée en droit - notamment - par Kelsen ${ }^{5}$. Elle a conduit à d'indéniables découvertes et elle constitue une base solide de raisonnement, mais elle n'est pas pour autant irréprochable. Particulièrement, la dynamique de spécialisation cartésienne présente l'inconvénient de fragmenter les savoirs, de plus en plus rapidement ${ }^{6}$ et à tel point qu'il est difficile aujourd'hui de se représenter les phénomènes dans leur globalité. Appliquée à la question du sol, la conception cartésienne nous aurait amené à disjoindre réalité biologique et réalité juridique, puis à réduire chacun de ces champs en diverses spécialisations non connectées.

Les auteurs des différents chapitres ont au contraire souhaité s'inscrire dans le courant de pensée dit « de la complexité » porté par Edgar Morin ${ }^{7}$. Son idée centrale est la suivante : pour mieux comprendre un objet d'étude, il faut l'examiner dans son entièreté et son environnement. Les nouvelles connaissances acquises grâce à cette mise en perspective de nombreuses données permettent de retrouver une cohérence entre des observations qui, prises isolément,

2. R. Descartes, 1637, Discours de la Méthode, p. 14.

3. C. Atias, 2002, L'épistémologie juridique, Dalloz, pp. 34 et ss.

4. A. Comte, 1830-1842, Cours de philosophie positive, Première et seconde leçons, http:// classiques.uqac.ca/classiques/Comte_auguste/cours_philo_positive/cours_philo_pos_1_2. pdf, spéc. pp. 52 et ss. et pp. 62 et ss..

5. H. Kelsen, 1999, Théorie pure du droit, Bruylant.

6. R. Boyer, «L'économie en crise : le prix de l'oubli de l'économie politique » in L'Économie politique, 2010/3, $\mathrm{n}^{\circ} 47$, p. 46.

7. Pour une approche complète cf. E. Morin E., 2008, La Méthode, Éd. du Seuil. 
semblaient contradictoires. "Adopter le paradigme de complexité [permet] de concevoir comme lié ce qui, jusqu'ici, était considéré comme disjoint $»^{8}$. L'approche complexe n'est certes pas parfaite. Prétendre à une recherche globale, à une recherche sur le " tout », peut se heurter notamment aux contingences matérielles du chercheur. En l'occurrence, notre approche du sol s'est concentrée sur les champs disciplinaires du droit et de l'agronomie, ce qui ne couvre assurément pas « son entièreté ». Les auteurs ont néanmoins estimé que les connaissances gagnées par cette " petite » entrée dans la complexité étaient importantes et que nous aurions perdu beaucoup en y renonçant.

Si cette perception de la démarche scientifique est désormais admise dans un certain nombre de disciplines, elle demeure relativement rare en droit. Comme la grande majorité de chercheurs en droit, nous nous considérons comme positivistes dans la mesure où les démonstrations partiront toujours d'une analyse du droit positif. Il s'agira d'étudier la norme existante, support observable ${ }^{9}$. Cependant, au sein des positivistes, nombre d'auteurs adhèrent à une conception normativiste du droit et notre démarche s'éloigne alors de la leur. En effet, les normativistes considèrent que pour être valide, une analyse doit se limiter à l'observation de la norme et se défaire des faits l'entourant ${ }^{10}$. Ils savent que le droit est le résultat de choix politiques eux-mêmes assis sur de très nombreuses justifications mais ils décident de ne pas intégrer ces considérations à leur réflexion par souci d'exactitude ${ }^{11}$. Ils s'inscrivent donc dans une logique de spécialisation telle que précédemment décrite ${ }^{12}$.

8. A. Ait Abdelmalek, «Edgar Morin, sociologue et théoricien de la complexité », Sociétés, $2004 / 4, n^{\circ} 188$, p. 115.

9. X. Magnon, 2008, Théorie(s) du droit, Ellipses, p. 16.

10. Pour ceux-ci, cette caractéristique fait aussi la particularité de la recherche en droit: « l'autonomie du discours juridique est manifeste par rapport à la (...) sociologie ou la science politique dans la mesure où le fait est écarté du domaine d'étude », X. Magnon, «En quoi le positivisme - normativisme - est-il diabolique ?», RTD civ., 2009, pp. 1 et ss. 11. E. Millard, 2006, Théorie générale du droit, Dalloz, p. 29.

12. Il faut préciser que, parmi les positivistes, les juristes adhérant à des épistémologies réalistes acceptent d'intégrer les faits à leurs travaux. Cependant, dans le cas du réalisme américain et du réalisme scandinave, à savoir les deux principaux sous-courants, l'empirisme passe avant tout par une étude des décisions prétoriennes, X. Magnon, Théorie(s) du droit, précité, p. 138. Si les décisions de justice seront traitées dans les développements à venir, l'intégration des faits ne se limitera pas à cette dimension jurisprudentielle. 
Notre approche diffère d'un certain nombre d'études juridiques en un second point. Elles font généralement de la neutralité axiologique $^{13}$ une exigence non négociable ; à défaut les propos ne seraient pas objectifs et la science verserait dans la théorie de la justice. Or, les contributeurs de cet ouvrage s'assignent la mission d'observer les énoncés prescriptifs de façon aussi objective que possible, mais aussi de dégager des voies d'amélioration de la protection des sols. L'ouvrage s'apparente donc à un travail de jurisprudence au sens aristotélicien ${ }^{14}$ puisqu'il commence par une étude de l'être pour tendre vers le devoir-être. Pour minoritaire que demeure notre approche complexe et finaliste, elle semble devenir de plus en plus courante ${ }^{15}$, et peut-être encore davantage en droit de l'environnement ${ }^{16}$.

13. M. Weber, «Essai sur le sens de la "neutralité axiologique" dans les sciences sociologiques et économiques » in Essais sur la théorie de la science, 1965, Plon, pp. 475-526. 14. P. Aubenque, La prudence chez Aristote, 2014, PUF.

15. M.-K. Daoust, « Repenser la neutralité axiologique, Objectivité, autonomie et délibération publique », Revue européenne des sciences sociales, 2015, n 53-1, pp. 199-225.

16. Sur l'approche complexe, voir par exemple, E. Gaillard, "Pour une approche systémique, complexe et prospective des droits de l'homme ", in Changements environnementaux globaux et droits de l'homme, C. Cournil et C. Colard-Fabregoule (dir.), 2012, Bruylant, 648 pages, pp. 49-69. Quant à l'aspect finaliste, les liens entre droit de l'environnement et théorie de la justice semblent structurels. Ils ont existé dès les premières études environnementales aux Etats-Unis au début du XX $\mathrm{XX}^{\mathrm{eme}}$ siècle, M. Torre-Schaub, «Quelques apports à l'étude de la notion de justice environnementale », in Changements environnementaux op. cit., pp. 71-87, spéc. p. 73. 


\title{
La protection du sol en droit
}

\author{
Carole HERMON \\ Professeure, Université Toulouse Capitole \\ IEJUC, F-31000
}

Le sol est un angle mort du droit. S'il est pris en compte dans certains de ses aspects, il n'est pas appréhendé dans son entièreté, comme un ensemble complexe aux fonctions diverses ${ }^{1}$.

C'est au sein du droit de l'environnement ${ }^{2}$ qu'aurait pu trouver place un corpus normatif cohérent et global relatif à la protection du sol en sa qualité de ressource naturelle. Singulièrement, ce «droit du sol » aurait pu être codifié sous le livre deuxième du Code de l'environnement consacré aux « milieux physiques », constitué de deux titres, l'un relatif à « l'eau, aux milieux aquatiques et marins », l'autre à « l'air et à l'atmosphère ». Il n'en est rien et l'absence de ce titre troisième consacré au sol ne peut qu'être remarquée ${ }^{3}$.

1. «Les fonctions écologiques des sols s'entendent du rôle qu'ils jouent au sein des écosystèmes, tel que, par exemple : servir de vivier à la biodiversité, contribuer au stockage, au filtrage et à la transformation d'éléments nutritifs, de substances et d'eau, à la recharge des nappes souterraines, à la séquestration du carbone ou encore à la régulation du carbone », Neyret L. et Martin G.-J., Nomenclature des préjudices environnementaux, LGDJ, 2012, p. 16.

2. Le droit de l'environnement est défini comme « le droit relatif à l'environnement ", (Prieur M. et al., Droit de l'environnement, Dalloz, $7^{\mathrm{e}}$ ed., 2016, $\mathrm{n}^{\circ}$ 9) et donc, le cas échéant, relatif au sol, et/ou comme le droit qui a « pour objet d'organiser (la) protection (de l'environnement) », Van Lang A., Droit de l'environnement, PUF, $4^{\mathrm{e}}$ ed., 2016, $\mathrm{n}^{\circ}$ 64, Prieur M. et al. Droit de l'environnement, précité, $\mathrm{n}^{\circ} 9$, et donc notamment, la protection du sol.

3. Cette absence est soulignée dans le rapport de Bellec P., Lavarde P., Lefèbvre L. et Madignier M.-L., Propositions pour un cadre national de gestion durable des sols, CGEDD-CGAAER, sep. 2015, p. 7. Relevant également en ce sens « le contraste existant entre la maturité du droit applicable à la protection de la qualité de l'eau et des milieux aquatiques et le caractère embryonnaire de celui dédié à la protection de la qualité du sol », Farinetti A., "La protection juridique de la qualité du sol au prisme du droit de l'eau », Env. et $D D, 2013, \mathrm{n}^{\circ}$ 6, Etude 17, ou « le délaissement du sol par le droit », Billet P. « La prise 
Par comparaison, l'eau et l'air font l'objet d'une protection large. On entend par là que le droit, sans vraisemblablement être exhaustif et sans augurer de son effectivité, comprend divers critères pour définir leur qualité et prescrit une protection de l'eau et de l'air en tant qu'ils constituent des ressources satisfaisant aux besoins de l'Homme, et en tant que milieu, quoi qu'il en soit de leurs usages par l'Homme.

S'agissant de l'eau, la qualité de l'eau destinée à la consommation alimentaire est définie et protégée par un ensemble de règles ${ }^{4}$. Le droit veille également à ce que les volumes et débits d'eau disponibles puissent satisfaire les différentes utilisations, récréationnelles, économiques et alimentaires, tout en préservant le milieu aquatique ${ }^{5}$. Au surplus, un objectif de «bon état» des eaux, correspondant à un bon état chimique ${ }^{6}$ et à un bon état écologique ${ }^{7}$ est imposé par le droit de l'Union européenne, et poursuivi, en droit interne, par les schémas d'aménagement et de gestion de l'eau'.

La structure du droit relatif à l'air est comparable. L'ensemble est constitué de normes fixant des valeurs à atteindre ou à ne pas dépasser ${ }^{9}$, et lorsqu'il apparaît qu'elles ne sont pas respectées ou risquent de ne pas l'être, doivent être mises en œuvre des mesures de nature à ramener la concentration en polluants à un niveau

en compte de la qualité des sols dans le droit français » in Bispo A., Guellier C., Martin E., Sapijanskas J., Soubelet H. et Chenu C. (coord.), Les sols. Intégrer leur multifonctionnalité pour une gestion durable, Quae, coll. Savoir Faire, 2016, p. 259.

4. Des critères de potabilité de l'eau sont posés (art. R. 1321-2 et 3 CSP), la production et la distribution d'eau en vue de la consommation humaine sont soumises à autorisation préfectorale (art. L. 1321-7 CSP), des contrôles sont ensuite organisés (art. L. 1321-4 et 5 CSP). Les points de captage d'eau sont également protégés par des servitudes d'utilité publique (art. L. 1321-2 CSP) ainsi que les aires d'alimentation des captages d'eau potable d'une importance particulière (art. L. $211-3-5^{\circ}$ et $7^{\circ}$ C. env.).

5. Art. L. 211-1-II C. env. A cette fin, ont été instituées des autorisations préalables de prélèvement (art. L. 214-1 et R. 214-1 C. env.), et des outils de gestion de crise pour répondre aux situations où la ressource est insuffisante : schémas d'aménagement et de gestion des eaux (art. L. 212-3 et L. 212-5-1-II- $1^{\circ}$ C. env.), autorisations uniques et organismes uniques de gestion collective (art. R. 211-111 et ss, art. R. 214-31-1 et ss. C. env.), arrêtés sécheresse (art. R. 211-66 C. env.).

6. Les concentrations de polluants sont inférieures à certains seuils.

7. Défini comme présentant une bonne «qualité de la structure et du fonctionnement des écosystèmes aquatiques $»$.

8. Directive cadre sur l'eau n 2000/60/CE du 23 octobre 2000 et art. L. 212-1-IV C. env.

9. Art. R. 221-1-II C. env. 
réglementaire ${ }^{10}$. Le tout est construit en référence à la protection de la santé et de l'environnement ${ }^{11}$, de sorte que la qualité de l'air ne porte pas atteinte à la santé humaine, conformément au «droit reconnu à chacun à respirer un air qui ne nuise pas à sa santé » (art. L. 220-1 C. env.), mais également ne comporte pas d'effets nocifs pour la végétation et les écosystèmes ${ }^{12}$.

Rien de tel à la faveur des sols. Le droit ne pose pas de critères de la qualité ${ }^{13}$ ou de la santé des sols, pas (ou guère ${ }^{14}$ ) de valeurs limites à ne pas dépasser pour préserver cette qualité ou de « valeursobjectifs » à atteindre; plus spécifiquement, il ne comporte pas de critères ou de valeurs à atteindre pour la qualité des sols agricoles. Sans qu'il soit pour l'heure question d'envisager les motifs de cette absence ${ }^{15}$, le constat en sera ici fait et la consistance de l'absence, mesurée.

En effet, même si ses qualités et son bon fonctionnement ne sont pas définis, cela ne signifie pas qu'il n'y a pas de protection du sol organisée ou induite par le droit. Le sol est pour partie protégé, «par

10. Ces mesures touchent l'usage des carburants ou combustibles, des véhicules, le fonctionnement et l'exploitation des installations polluantes, le contrôle de ces installations (art. L. 222-5 al. 3, art. L. 226 C. env.), et comprennent le cas échéant des mesures d'urgence en cas de pic de pollution (art. L. 223-1 C. env.).

11. Cf. art. L. 220-2, L. 221-1, L. 221-2, L. 221-6, art. R. 221-1-II, R. 222-2 C. env.

12. Cf. art. 2 Protocole de Göteborg relatif à la réduction de l'acidification, de l'eutrophisation et de l'ozone troposphérique du 30 novembre 1999, art. $1^{\text {er }}$ directives 2001/81/CE du 23 octobre 2001 et 2016/2284/UE du 14 décembre 2016 relatives aux plafonds des émissions nationales de certains polluants atmosphériques.

13. En ce sens Desrousseaux M., La protection juridique de la qualité des sols, LGDJ, bibliothèque de droit de l'urbanisme et de l'environnement, t. 13, 2016, $\mathrm{n}^{\circ} 25:$ : La traduction juridique de (la) qualité (des sols) ne se retrouve... ni en droit international, ni en droit européen, ni en droit interne, alors qu'aux yeux du monde scientifique, elle "apparaît comme la méthode garantissant au mieux la mise en œuvre d'une politique enfin efficace pour la protection des sols" ».

14. Les textes relatifs à l'épandage à fins agricoles des boues issues du traitement des eaux usées font figure d'exception. L'article 6 du décret n $97-1133$ du 8 décembre 1997, adopté pour transposition de la directive 86-278 du 12 juin 1986, dispose que l'épandage des boues ne doit pas porter atteinte, notamment, "à la qualité des sols ", et à cette fin, renvoie à un arrêté ministériel le soin de fixer « les teneurs maximales en éléments traces et composés organiques traces présents dans les boues » (art. 15), arrêté adopté le 8 janvier 1998, arrêté fixant les prescriptions techniques applicables aux épandages de boues sur les sols agricoles pris en application du décret n ${ }^{\circ} 97-1133$ du 8 décembre 1997 relatif à l'épandage des boues issues du traitement des eaux usées.

15. Cf. infra Chabert A. et Sarthou J.-P. « Le sol agricole, une ressource indispensable négligée », Bosc L. " Propriété et protection des sols. Réflexions civilistes sur la prise en compte de la qualité des sols ». 
ricochet ${ }^{16}$, par une législation dont la protection des sols n'est pas l'objet mais qui, néanmoins, permet de déployer une certaine protection (I). Il est également protégé par une législation dont tel est l'effet recherché, mais cette législation, constituée de normes éparses et ponctuelles, ne garantit qu'une protection fragmentaire des sols (II). De sorte que la question de la construction d'un « droit du sol » demeure posée (III).

\section{LA PROTECTION DU SOL PAR LE DROIT, EFFET RICOCHET}

Les normes dont l'objet n'est pas la protection du sol mais concourant à la protection des sols, sont nombreuses ${ }^{17}$ du fait des interactions que nourrit le sol avec les autres ressources naturelles, les plantes, animaux et micro-organismes d'une part, avec les activités de l'homme, et particulièrement les activités agricoles d'autre part. Ainsi, en premier lieu, en protégeant une composante de l'environnement autre que le sol, ou en préservant un usage supporté par le sol, certaines règles de droit conduisent indirectement à une protection du sol, même si tel n'est pas l'objectif poursuivi par la norme (A). En second lieu, le sol peut être protégé non pas spécifiquement mais comme une composante de l'environnement (B).

\section{A. LA PROTECTION INDIRECTE DU SOL}

La protection que peut indirectement offrir le droit au sol peut être illustrée par divers exemples. Nous en exposerons trois. D'une part, dans la mesure où le sol fonctionne en lien avec l'eau et les organismes vivants qu'il supporte, la protection apportée par le droit à l'eau (1), aux espèces et aux écosystèmes (2), peut bénéficier au sol par ricochet. D'autre part, la protection des zones affectées à l'agriculture contre les usages concurrents préserve également les sols contre l'artificialisation liée au développement de l'urbanisation et de l'imperméabilisation qui en résulte (3).

16. Selon l'expression de Desrousseaux M., La protection juridique de la qualité des sols, précité, $\mathrm{n}^{\circ} 501$.

17. A ce titre, l'examen qui suit n'est pas exhaustif mais s'appuie sur des exemples étayant le propos. 


\section{1) Droit de l'eau et sol}

Le droit de l'eau constitue une première illustration de ce que peut recouvrir une protection indirecte des sols. La protection de la qualité de l'eau en général, de la qualité de l'eau destinée à l'alimentation humaine en particulier, comprend des dispositions destinées à prévenir les risques de ruissellement et d'infiltration de substances polluantes dans l'eau. Ainsi peuvent être prescrites la couverture des sols pendant les périodes présentant des risques de lessivage ou l'implantation d'une bande enherbée le long des cours d'eau ${ }^{18}$. L'apport de fertilisants et produits phytosanitaires, le travail du sol ou la gestion de l'interculture peuvent également être réglementés ${ }^{19}$. Ce faisant, même si tel n'est pas l'objet premier de ces dispositions, le droit de l'eau impose des mesures bénéfiques à la qualité et à la santé des sols, en prévenant leur contamination et en limitant les pertes de sol et de matières organiques par érosion.

\section{2) Protection des écosystèmes et des espèces et sol}

De même, en poursuivant un objectif de protection des écosystèmes et des espèces, le droit peut comprendre des dispositions de nature à préserver la qualité des sols et leur bon fonctionnement. Exemple peut être pris des zones Natura 2000, constituées d'habitats naturels remarquables, menacés, abritant des espèces de la faune ou de la flore rares ou vulnérables, et de sites particuliers de passage,

18. Ceci est rendu obligatoire, sauf adaptations régionales, depuis 2009 dans toutes les « zones vulnérables », c'est-à-dire les zones affectées par une nitrification de l'eau ou une euthrophisation de l'eau du fait de l'usage des fertilisants sur les sols agricoles, art. R. 21175 et ss C. env. $C f$. art. R. $211-81-7^{\circ}$ et $8^{\circ}$ C. env., arrêté modifié du 19 décembre 2011 relatif au programme d'actions national à mettre en oeuvre dans les zones vulnérables afin de réduire la pollution des eaux par les nitrates d'origine agricole, annexe I-VII et VIII, adopté pour transposition de la directive n ${ }^{\circ}$ 91/676 du 12 décembre 1991 concernant la protection des eaux contre les nitrates à partir de sources agricoles.

19. L'apport d'azote organique est limité dans toutes les zones vulnérables (cf. note précédente pour la définition), art. R. $211-81-5^{\circ} \mathrm{C}$. env. Par ailleurs, dans les périmètres de protection rapprochée des points de captage d'eau destinée à l'alimentation humaine, sont interdites les activités " de nature à rendre l'eau impropre à la consommation humaine » et réglementées celles qui peuvent avoir un impact sur la ressource (art. R. 132113 al. 3 CSP), ce qui peut viser les apports d'intrants, le travail du sol ou la gestion de l'interculture. Au surplus, dans les aires d'alimentation de captage d'eau potable d'une importance particulière (art. L. 211-3-5 ${ }^{\circ}$ C. env.), des « zones soumises à contraintes environnementales " peuvent être délimitées à l'intérieur desquelles seront prescrites ou proposées par voie contractuelle : des mesures de couverture du sol, de travail du sol, de gestion des intrants, de diversification et rotation des cultures (art. R. 114-6 C. rural). 
de repos ou de reproduction de certaines espèces d'oiseaux (art. L. 411-1 C. env.). Dans ces zones, doivent être mises en œuvre des mesures propres à conserver les habitats en bon état et à prévenir leur dégradation, ainsi que l'atteinte aux espèces de faune et de flore présentes (art. L. 411-1-V C. env.). Pour ce faire, d'une part des contrats sont établis avec les propriétaires et exploitants des terrains inclus dans le $\operatorname{site}^{20}$ (art. L. 414-3 C. env.), et d'autre part, l'essentiel des activités susceptibles d'affecter de manière significative un site Natura 2000 doivent faire l'objet d'une évaluation des incidences, y compris celles qui ne relèvent en principe d'aucune autorisation (art. L. 414-4 C. env.). A ces deux titres les sols peuvent bénéficier d'une protection, même si tel n'est pas l'objet de la norme. Les contrats Natura 2000 peuvent interdire l'usage des fertilisants, des produits phytosanitaires, le retournement des prairies, imposer la création et l'entretien d'un couvert, l'entretien des haies, de bosquets, prescrire un chargement maximal ou minimal pour éviter sur-pâturage ou sous-pâturage... Quant aux activités qui relèvent de l'étude d'incidence et d'une autorisation ad hoc, alors qu'elles y échapperaient en droit commun, on en trouve dont le contrôle bénéficie assurément, outre à l'habitat et aux espèces, aux sols, à savoir : l'épandage de boues, d'effluents, le retournement de prairies ou de landes et l'arrachage de haies (art. R. 414-27 C. env.).

Pareillement, la protection des zones agricoles peut permettre, par ricochet, que soient préservés certains sols, à tout le moins d'un risque particulier, celui de l'urbanisation.

\section{3) Protection des zones agricoles et sol}

Le droit entend répondre au phénomène continu de perte de foncier agricole par une protection des espaces agricoles contre l'étalement urbain. Depuis la loi SRU ${ }^{21}$, le législateur affiche l'ambition ${ }^{22}$ de « construire la ville sur la ville » et, plus précisément, en application

20. Ces contrats peuvent prendre la forme d'une MAEC (mesure agro-environnementale et climatique) lorsqu'ils sont conclus par les exploitants agricoles.

21. Loi n 2000-1208 du 13 déc. 2000 relative à la solidarité et au renouvellement urbains.

22. Etant entendu que cette ambition est largement tenue en échec $C f$. en ce sens la conclusion de Desrousseaux M., thèse précitée, $\mathrm{n}^{\circ} 367$ : «le droit reste trop faible pour contrevenir au processus de raréfaction dans lequel la ressource sol est engagée ». 
de la loi Grenelle $2^{23}$, les documents d'urbanisme doivent dorénavant fixer des objectifs de protection des espaces agricoles et de lutte contre l'urbanisation continue ${ }^{24}$. Il n'y a dans les diverses règles adoptées à ce titre, aucune visée de protection du sol per se, mais une volonté de préserver des espaces voués à l'agriculture, c'està-dire de préserver l'activité agricole, quelle qu'elle soit, en lui affectant certaines zones. Pour autant, en encadrant les possibilités de changement d'affectation des zones agricoles ${ }^{25}$ et en restreignant fortement les possibilités de construire dans ces zones ${ }^{26}$, le droit de l'urbanisme protège les sols de l'imperméabilisation consécutive à l'urbanisation ${ }^{27}$.

La protection du sol peut également résulter par ricochet d'une législation qui prétend embrasser l'environnement en général.

\section{B. La protection des sols fondue DANS la protection de L'ENVIRONNEMENT}

Le droit de l'environnement comprend des corps de règles transversales qui tendent à la protection de l'ensemble des composantes de l'environnement, et donc notamment à la protection des sols. Tel est le cas de certaines procédures environnementales (exemple peut être pris de l'étude d'impact) et de certaines polices ${ }^{28}$ environnementales (exemple pris du droit des installations classées).

23. Loi n ${ }^{\circ} 2010-788$ du 12 juillet 2010 portant engagement national pour l'environnement.

24. Ces objectifs doivent être fixés par les plans d'aménagement et de développement durable des SCOT (schémas de cohérence territoriale) et des PLU (plans locaux d'urbanisme) (art. L. 141-2 et L. 151-5 C. urb.) et ils doivent être chiffrés (art. L. 141-6 et L. 151-5 C. urb.).

25. Particulièrement dans les communes non couvertes par un SCOT où les zones agricoles ne peuvent être ouvertes à l'urbanisation, art. L. 142-4 C. urb.

26. Ne sont pour l'essentiel autorisées en zones agricoles que les constructions et installations nécessaires aux services publics ou d'intérêt collectif et à l'exploitation agricole (art. L. 111-4, L. 161-4 et R 151-23 C. urb.).

27. Aucune règle d'urbanisme ne peut en revanche venir imposer des méthodes de production à un agriculteur ou en interdire d'autres parce qu'elles porteraient atteinte à la qualité ou à la santé des sols.

28. Par «police », sous-entendue administrative, on entend " l'activité spécifique de prescription, consistant à réglementer des activités privées en vue du maintien de l'ordre public » (Van Lang A., Gondouin G., Inserguet-Brisset V., Dictionnaire de droit administratif, Sirey, $5^{\mathrm{e}}$ ed, 2008), certaines polices (on parle alors de police spéciale) pouvant avoir une finalité propre, ainsi la protection de l'environnement en ce qui concerne la police environnementale. 


\section{1) Etude d'impact et sol}

La procédure de l'étude d'impact, précédant les travaux et projets d'aménagement susceptibles de porter atteinte à l'environnement, a été instituée par la loi $\mathrm{n}^{\circ} 76-629$ du 10 juillet 1976 relative à la protection de la nature. Elle doit permettre en premier lieu au pétitionnaire de mesurer les effets de son projet sur l'environnement et en conséquence, de les prévenir, de les réduire voire de les compenser, et à l'autorité administrative en second lieu de s'assurer que le projet ne porte pas d'atteinte excessive à l'environnement. Aussi les textes exigent-t-ils de l'étude d'impact qu'elle présente une analyse large de tous les effets, directs et indirects, que le projet comporte pour l'ensemble des composantes de l'environnement ${ }^{29}$.

Si les textes initiaux ne comportaient pas de référence expresse aux sols, le décret $\mathrm{n}^{\circ}$ 93-245 du 25 février 1993, modifiant le contenu obligatoire de l'analyse des effets du projet sur l'environnement, l'ajoute à l'énumération. Depuis lors, le contenu des études d'impact a été modifié ${ }^{30}$, mais l'obligation d'analyser les effets des projets sur les sols est demeurée et elle a été précisée par un décret du 11 août $2016^{31}$.

Fondue dans le tout, on ne peut toutefois augurer que, en pratique, l'analyse des impacts sur les sols soit toujours parfaitement menée mais il reste que, en droit, le juge administratif peut annuler l'autorisation administrative irrégulièrement délivrée, en l'absence d'analyse - ou d'analyse suffisante - des effets du projet sur les sols ${ }^{32}$.

29. En l'état actuel des textes, l'étude d'impact doit porter sur les facteurs suivants : « la population, la santé humaine, la biodiversité, les terres, le sol, l'eau, l'air, le climat, les biens matériels, le patrimoine culturel, y compris les aspects architecturaux et archéologiques, et le paysage », art. R. $122-5-$ II $-4^{\circ} \mathrm{C}$. env.

30. Décrets n 2011-2019 du 29 décembre 2011 et n 2016-1110 du 11 août 2016, codifiés sous l'article R. 122-5 C. env..

31. L'étude d'impact doit comporter : « une estimation des types et des quantités de résidus et d'émissions attendus, tels que la pollution de l'eau, de l'air, du sol... » et une estimation des « facteurs susceptibles d'être affectés par le projet», dont le sol, art. R. 122-5-II- $2^{\circ}$ C. env..

32. En ce sens : TA Nice 20 avril 1995 Chabas et autres c. Commune du Val, RJE 1996, p. 158 : annulation de l'autorisation de créer une ZAC comprenant un village de vacances avec golf; « l'étude d'impact ne comprend aucune analyse des effets... du projet sur le sol et l'eau », TA Strasbourg 18 juillet 1997 ANEED c. Préfet de la Moselle, RJE 1999, p. 275 : annulation de l'autorisation d'exploiter un centre de stockage de déchets industriels, "l'étude d'impact ne comporte aucune indication sur les conséquences du projet sur le sol, sur la faune et la flore, sur le paysage, sur l'approvisionnement en eau et sur la population », 
Le droit des installations classées ${ }^{33}$ qui tend à encadrer tous les risques et inconvénients que créent lesdites installations pour l'environnement, peut pareillement prévenir ou sanctionner une atteinte portée aux sols.

\section{2) Droit des installations classées et sol}

La question de la pollution des sols est centrale lorsqu'une installation classée est mise à l'arrêt définitif ; leur remise en état est alors l'objet même du droit (cf. infra II.B.). Elle est moins prégnante au stade de la délivrance de l'autorisation d'exploiter.

Néanmoins, aux termes de l'article L. 181-12 du Code de l'environnement, l'autorisation ne peut être régulièrement accordée que si elle est accompagnée des prescriptions de nature à prévenir ou réduire les « effets négatifs notables (de l'exploitation) sur l'environnement et la santé », ce qui recouvre assurément les sols. Au surplus, l'énumération des intérêts protégés par la législation relative aux installations classées ${ }^{34}$, au regard desquels l'autorisation doit être prise, est suffisamment large pour pouvoir comprendre des enjeux liés aux sols, même si ceux-ci n'y figurent pas expressément. Au demeurant, pour certaines installations classées, la prise en compte du risque pour les sols est rendue obligatoire, à savoir pour les établissements qui ont les plus gros volumes de stockage ou de production, et relèvent à ce titre de la directive $\mathrm{IED}^{35}$, et pour des

CAA Paris 16 avril 1998 Société Sovetra, req. n 96PA01543 : annulation de la délibération approuvant la création d'une zone d'aménagement concertée, l'étude d'impact « ne fait pas apparaître avec précision les conséquences de ce projet sur l'environnement, en ce qui concerne en particulier ses effets sur l'eau et sur le sol ». Cette jurisprudence demeure toutefois marginale au regard de celle sanctionnant l'insuffisance des analyses sur l'eau ou la biodiversité.

33. Constitue une installation classée, l'installation industrielle ou agricole qui présente des risques ou inconvénients pour l'environnement et/ou le voisinage et est, à ce titre, inscrite sous une nomenclature adoptée par décret en Conseil d'Etat, annexée à l'article R. 511-9 du Code de l'environnement.

34. Sont énumérés sous l'article L. 511-1 C. env. : « la commodité du voisinage, la santé, la sécurité, la salubrité publiques, l'agriculture, la protection de la nature, de l'environnement et des paysages, l'utilisation rationnelle de l'énergie, la conservation des sites et des monuments, les éléments du patrimoine archéologique ».

35. Directive 2010/75/UE du 24 novembre 2010 relative aux émissions industrielles. Selon l'article R. 515-60 du Code de l'environnement, pour ces installations, " l'arrêté d'autorisation fixe au minimum... e) des prescriptions garantissant la protection du sol, f) des prescriptions concernant la surveillance périodique du sol (lorsque l'activité implique l'utilisation, la production ou le rejet de substances ou de mélanges dangereux) ». 
installations classées particulières pour lesquelles l'arrêté ministériel fixant les prescriptions générales de fonctionnement comprend des dispositions spécifiques aux sols ${ }^{36}$.

In fine, on ne peut négliger les bénéfices d'une législation qui assure par ricochet une protection des sols, indirectement (la protection des sols ne constituant pas l'objet de la norme), ou par inclusion (la protection des sols constituant un des objets de la norme parmi d'autres). Les exemples cités montrent qu'elle contribue à la prévention des risques qui menacent les sols, érosion, perte de matières organiques et de biodiversité, pollution et imperméabilisation. Mais on ne peut néanmoins attendre d'une législation qui ne tend pas, ou pas spécifiquement, à la protection des sols les effets et l'efficacité37 d'une législation ad hoc dont l'objectif serait la protection des sols.

Une telle législation existe. Elle demeure toutefois aujourd'hui incomplète.

\section{LE DROIT CONSACRÉ À LA PROTECTION DU SOL, UNE PROTECTION FRAGMENTAIRE}

Il existe en droit certaines normes qui ont pour objectif de protéger les sols et instituent effectivement une protection des sols. Peuvent être distinguées, des dispositions qui tendent en premier lieu à prévenir ou limiter les dégradations des sols, et même plus particulièrement les dégradations des sols agricoles (A). En second lieu, obligation est parfois faite de réparer ou remettre en état des sols dégradés, sans

\footnotetext{
36. Par exemples, l'arrêté du 27 décembre 2013 fixant les prescriptions générales applicables aux élevages de bovins, volailles et porcs (régime de l'autorisation) prévoit des mesures contre la dégradation des sols occupés par les porcs élevés en plein air et les parcours des volailles (art. 20 et 21), des restrictions à l'épandage des effluents (art. 27-1) et des règles de stockage des déchets de l'élevage (art. 34). L'arrêté du 26 novembre 2012 relatif aux prescriptions générales de fonctionnement des silos de stockage de céréales (régime de l'enregistrement) prévoit également des règles de stockage en cas de risques de pollution des sols (art. 22). On peut toutefois relever que semblables prescriptions relatives au sols sont rares. L'article R. 512-72-1 du Code de l'environnement, tel que modifié en 2013, prévoit pourtant que « lorsque la protection des intérêts mentionnés à l'article L. 511-1 le justifie, le ministre chargé des installations classées fixe... les méthodes de diagnostic, de prévention, de traitement ou de réduction de la pollution des sols applicables respectivement aux différentes catégories d'installations classées ».

37. Est « efficace » la norme qui produit les effets recherchés, « les effets que l'on attendait d'elle », de Béchillon D., Qu'est-ce qu'une règle de droit ?, O. Jacob, 1997, p. 10.
} 
disposition spécifique ici sur la question des sols agricoles (B). Mais la juxtaposition de ces textes épars ne conduit qu'à une protection partielle des sols.

\section{A. LA PRÉVENTION DES ATTEINTES AU SOL}

Alors que « la prolifération des textes ", "l'inflation normative $»^{38}$ est souvent dénoncée, on ne peut, s'agissant de la prévention de la dégradation des sols ou de la limitation de ces dégradations, que relever la rareté du droit. Nous n'avons trouvé, en dehors des dispositions adoptées au titre des aides PAC qui constituent donc l'essentiel du dispositif normatif (5), que quatre corps de règles, le premier relatif à l'érosion des sols (1), le deuxième au changement d'affectation des sols en vue de la production de biocarburant (2), le troisième à l'épandage des boues de station d'épuration (3), le quatrième à la protection des sols dans les Alpes (4). Cette simple énumération révèle le caractère quasi anecdotique du droit prétendant prévenir les atteintes au sol.

\section{1) La protection contre l'érosion}

Le traitement de l'érosion des sols est apparu au début du vingtième siècle en droit forestier dans un but de prévention des risques naturels, risques d'inondations, de mouvements de terrain et d'avalanches ${ }^{39}$; il a été étendu en $2003^{40}$.

D'une part, la prévention de l'érosion est intégrée aux objectifs de la politique agricole ${ }^{41}$. D'autre part, est introduit dans le Code rural un nouveau chapitre intitulé : « l'agriculture de certaines zones soumises à des contraintes environnementales », ces zones étant constituées des

38. Cf. notamment Conseil d'Etat, Rapport public 1991, De la sécurité juridique, Rapport public 2006, Sécurité juridique et complexité du droit, Rapport de la mission de lutte contre l'inflation normative, Lambert A., Boulard J.-C., rapport au Premier ministre, 26 mars 2013.

39. Cf. Desrousseaux M., thèse précitée, $\mathrm{n}^{\circ} 520$ et ss., Fèvre M. « Les services écologiques et le droit. Une approche juridique des systèmes complexes », thèse Université Côte d'Azur, 12 déc. 2016, pp. 77-80.

40. Loi n $2003-699$ du 30 juillet 2003 relative à la prévention des risques technologiques et naturels et à la réparation des dommages.

41. Complément étant apporté à l'article $1^{\text {er }}$ de la loi n ${ }^{\circ} 99-574$ du 9 juillet 1999 d'orientation agricole : « La politique agricole... a pour objectifs .... la prévention... de l'érosion des sols ». 
« zones dites “zones d'érosion" »" sont déployés comprenant un ensemble de mesures ${ }^{43}$ de nature à remédier à l'érosion.

La portée de ce nouvel outil est toutefois limitée à deux titres. Les zones d'érosion doivent en premier lieu faire l'objet d'une délimitation administrative ${ }^{44}$ et les travaux parlementaires indiquent qu'elles n'ont pas vocation à recouvrir tout le territoire national affecté par l'érosion ${ }^{45}$. La finalité poursuivie par la délimitation des zones d'érosion et la définition desdites zones qui en découle, corroborent cette volonté restrictive de mise en œuvre des zones de contraintes environnementales. Est seule mise en exergue la prévention du risque d'inondation : l'érosion des sols doit être prévenue, ou limitée, parce qu'elle accentue les risques d'inondations ${ }^{46}$. Dès lors, les zones d'érosion « couvrent les parties du territoire où... les pratiques agricoles ont favorisé l'érosion des sols et l'accélération de l'écoulement des eaux de ruissellement qui ont été à l'origine de dommages causés en aval ou sont susceptibles

\section{Art. L. 114-1 C. rural.}

43. Le programme d'actions "définit les mesures... parmi les actions suivantes : $1^{\circ}$ couverture végétale du sol, permanente ou temporaire ; $2^{\circ}$ travail du sol, gestion des résidus de culture, apports de matière organique favorisant l'infiltration de l'eau et limitant le ruissellement $; 3^{\circ}$ gestion des intrants..., $4^{\circ}$ diversification des cultures...; $5^{\circ}$ maintien ou création de haies, talus murets...; $6^{\circ}$ restauration ou entretien d'un couvert végétal spécifique ; $7^{\circ}$ restauration ou entretien des mares, plans d'eau ou zones humides $"$, art. R. 114-6 C. rural.

44. Cette délimitation ressort de la compétence du préfet.

45. Etaient visées plus particulièrement les régions Normandie, Bourgogne, Bretagne et Pays de Loire, rapport Détraigne Y., doc. Sénat, n 154, 29 janv. 2004, p. 90. Vénot A., dans son rapport devant l'Assemblée Nationale, précisait : « il n'est nullement dans l'intention du Gouvernement de couvrir l'ensemble des départements de zones d'érosion, ces nouveaux outils devant être utilisés avec discernement », Rapport Assemblée Nationale au nom de la Commission des affaires économiques, de l'environnement et du territoire, 26 février $2003, n^{\circ} 635,1^{\text {re }}$ partie.

46. Le nouveau dispositif (art. 21 de la loi) est ainsi inscrit sous le titre III relatif aux risques naturels et la présentation du projet de loi par le Gouvernement corrèle érosion des sols et prévention des inondations. Cf. doc. Sénat, $n^{\circ} 116,3$ janvier 2003 : «certaines pratiques agricoles peuvent en effet favoriser l'érosion des sols et accélérer l'écoulement des eaux de ruissellement (sillons dans le sens de la pente, arrachage de haies, retournement des prairies, etc.) ». En ce sens également, Rapport Vénot A., Commission des affaires économiques, de l'environnement et du territoire, 26 février 2003, doc. $\mathrm{AN}, \mathrm{n}^{\circ} 635,1^{\text {re }}$ partie : « une politique de prévention des inondations efficace doit donc... lutter contre ces phénomènes d'érosion, en encourageant le recours à des pratiques agricoles appropriées. Tel est l'objet de l'article $21 \mathrm{du}$ projet de loi ». 
d'en causer $»^{47}$. La finalité de la protection des sols contre l'érosion a été élargie par la suite à la qualité de l'eau ${ }^{48}$. De sorte que la définition des zones d'érosion a été modifiée pour inclure les zones où : «... une érosion diffuse des sols agricoles (est) de nature à compromettre la réalisation des objectifs de bon état des eaux, ou le cas échéant de bon potentiel écologique ${ }^{49}$. Pour autant, la perte de substrat ou les atteintes à la biodiversité induites par l'érosion ne sont pas intégrées dans cette définition et ne constituent pas, en l'état, des motifs exprès de délimitation des zones d'érosion.

En second lieu, les zones d'érosion ont la singularité de pouvoir articuler dans le temps incitation puis contrainte. Le programme d'actions " visant à réduire l'érosion des sols de ces zones » comprend des pratiques « à promouvoir $»^{50}$ et des pratiques qui « peuvent être rendues obligatoires » (art. L. 114-1 C. rural). Si, «à l'expiration d'un délai de trois ans suivant la publication du programme d'action », sa mise en œuvre est insuffisante, le préfet peut « décider de rendre obligatoires, dans les délais et les conditions qu'il fixe, certaines des mesures préconisées par le programme » (art. R. 114-8 C. rural). Mais il n'est pas tenu de le faire.

C'est donc finalement la législation ancienne des forêts de protection, adoptée dès $1922^{51}$ " pour préserver l'intégrité des forêts jouant un rôle déterminant dans le maintien des sols et la lutte contre l'érosion $\|^{52}$, qui s'avère la plus contraignante. Peuvent être classées au titre des forêts de protection, notamment, « les bois et forêts dont la conservation est reconnue nécessaire au maintien des terres sur

\footnotetext{
47. Art. $1^{\text {er }}$ décret n ${ }^{\circ} 2005-117$ du 7 février 2005 relatif à la prévention de l'érosion, codifié sous l'article R. 114-1 du Code rural.

48. Art. 8 loi n 2012-1460 du 27 décembre 2012, modifiant l'article L. 211-3-II du Code de l'environnement : les programmes d'actions peuvent également être mis en œuvre dans les zones où l'érosion des sols agricoles « est de nature à compromettre la réalisation des objectifs de bon état ou, le cas échéant, de bon potentiel » de l'eau.

49. Décret $\mathrm{n}^{\circ}$ 2007-882 du 14 mai 2007 relatif à certaines zones soumises à contraintes environnementales codifié sous l'article R. 114-2 C. rural.

50. Au soutien des mesures incitatives, des aides peuvent être instituées «lorsqu'elles induisent des surcoûts ou des pertes de revenus » (art. L. 114-1 C. rural). Les rapports parlementaires précités, Vénot A. et Détraigne Y., soulignaient que ces mesures facultatives bénéficiant de financements publics pouvaient s'inscrire dans le cadre des mesures agro-environnementales ou des contrats territoriaux d'exploitation, alors en vigueur.

51. Loi du 28 avril 1922 relative aux forêts de protection, JO 4 mai 1922, p. 4606.

52. Liagre J. « Bois et forêts. Protection des bois et forêts », JCl. Env. et DD., fasc. 3720, $\mathrm{n}^{\circ} 138$, voir également, Fèvre M., thèse précitée, pp. 124-126.
} 
les montagnes et sur les pentes » et à la défense contre l'érosion ${ }^{53}$, ce qui représentait, en 2011, un peu plus de 63000 hectares $^{54}$. Dans ces zones, " tout changement d'affectation ou toute occupation de nature à compromettre la conservation ou la protection des boisements » est interdit ${ }^{55}$. L'exploitation de la forêt, de la ressource en eau et le pâturage y sont soumis à des conditions restrictives ${ }^{56}$. $\mathrm{Au}$-delà, le Code forestier institue également une protection des sols des forêts de montagne, même non classées au titre de l'article L. 141-1, au regard du risque érosif lié à la déclivité ${ }^{5}$. A minima, les communes doivent y établir un règlement des pâturages communaux (art. L. 142-5 C. for.). Le préfet peut également décider de la mise en défens de certains terrains et pâturages « à quelque propriétaire qu'ils appartiennent $»^{58}$. Et si « le maintien et la protection des terrains » l'exigent, des travaux de restauration et de reboisement peuvent être déclarés d'utilité publique (art. L. 142-7 C. for.).

\section{2) La protection des sols contre la production des biocarburants}

Face au potentiel développement des biocarburants ${ }^{59}$ et bioliquides $^{60}$, appelés à être utilisés pour satisfaire les objectifs nationaux de réduction des gaz à effet de serre et répondre à ceux définis en droit de l'Union européenne pour promouvoir les biocarburants ${ }^{61}$, le risque a rapidement été identifié d'une trop grande mise en culture

53. Art. L. 141-1 C. for.. Peuvent également être classés comme forêts de protection « les bois et forêts situés à la périphérie des grandes agglomérations, ... situés dans les zones où leur maintien s'impose pour des raisons écologiques (ou) pour le bien-être de la population»

54. Cf. Etude « Forêts » in CPEN, Elnet.

55. Art. L. 141-2 C. for.. Particulièrement, « aucun défrichement, aucune fouille, aucune extraction de matériaux, aucune emprise d'infrastructure publique ou privée, aucun exhaussement du sol ou dépôt ne peuvent être réalisés », art. R 141-14 C. for..

56. Art. L. 141-5 C. for..

57. $C f$. Desrousseaux M., thèse précitée, $n^{\circ}$ 510, Fèvre M., thèse précitée, pp. 119-122.

58. La période de mise en défens ne peut toutefois excéder dix ans et une indemnité sera alors due aux propriétaires, art. L. 142-1 et 2 C. for..

59. Par biocarburant, on entend « un combustible... utilisé pour le transport et produit à partir de la biomasse définie à l'article L. 211-2 », à savoir, « la fraction biodégradable des produits, déchets et résidus provenant de l'agriculture... ainsi que la fraction biodégradable des déchets industriels et ménagers ", art. L. 661-1 C. énergie.

60. Par bioliquide, on entend « un combustible liquide destiné à des usages énergétiques autres que le transport... et produit à partir de la biomasse », art. L. 661-1 C. énergie.

61. La directive 2003/30/CE du 8 mai 2003 fixe des objectifs d'incorporation des biocarburants et autres carburants renouvelables, de $2 \%$ au 31 décembre 2005 à $5,75 \%$ au 31 décembre 2010, art. 3 directive 2003/30/CE visant à promouvoir l'utilisation de biocarburants ou autres carburants renouvelables dans les transports. 
de sols aux fins de production de matières premières destinées à ces biocarburants et bioliquides, et de retournement de sols naturels pour affectation à ladite production ${ }^{62}$. L'antagonisme entre protection des sols et production de biocarburants a été rappelé par le Parlement européen dans une résolution du 29 septembre 2011 : « les sols sont une ressource rare » et le Parlement « appelle de ses vœux des actions concrètes et efficaces et des mesures de contrôle, notamment pour ce qui concerne la production de biocarburants $»^{63}$.

Aussi, ont été arrêtés des critères dits de « durabilité » de la production relatifs aux sols, critères destinés à protéger la biodiversité et le stockage de carbone par les sols ${ }^{64}$. Seuls sont dorénavant pris en compte pour le respect des objectifs de développement des énergies renouvelables (art L. 661-2 al. 1 C. énergie), les biocarburants et bioliquides respectant lesdits critères de durabilité. Les avantages fiscaux et autres aides publiques en faveur des biocarburants sont également subordonnés au respect de ces critères (art. L. 661-2 al. $2 \mathrm{C}$. énergie). Au surplus, une nouvelle directive est venue modifier les textes précédents pour limiter les changements d'affectation des sols, y compris indirects ${ }^{65}$, à des fins de production de biocarburants ${ }^{66}$.

62. On parle d'effet ILUC, indirect land use change, pour plus de développements, cf. Desrousseaux M., La protection juridique de la qualité des sols, thèse précitée, p. 360, $\mathrm{n}^{\circ} 761$, « Biocarburants », Etude CPEN, Elnet.

63. Résolution sur l'élaboration d'une position commune de l'Union dans la perspective de la conférence des Nations-Unies sur le développement durable (Rio+20), P7 TA (2011)0430, §52.

64. Directives 2009/28/CE et 2009/30/CE du 23 avril 2009 (respectivement, art. 17 et 7 ter); aux termes de l'article L. 661-5 du Code de l'énergie, «les biocarburants et bioliquides ne doivent pas être produits à partir de matières premières qui proviennent : $1^{\circ}$ De terres de grande valeur en termes de biodiversité ; $2^{\circ}$ De terres présentant un important stock de carbone ; $3^{\circ}$ De terres ayant le caractère de tourbières $»$, celles-ci constituant au demeurant de véritables réservoirs écologiques et une importante source de stockage de carbone.

65. Directive 2015/1513 du 9 septembre 2015; aux termes du considérant 9 de la directive, " lorsque des pâturages ou des terres agricoles destinés auparavant aux marchés de l'alimentation humaine ou animale sont convertis pour la production de biocarburants, la demande de produits autres que le carburant devra néanmoins être satisfaite, soit par l'intensification de la production actuelle, soit par la mise en production d'autres terres non agricoles. Ce dernier cas constitue un changement indirect dans l'affectation des sols et, lorsqu'il s'agit de la conversion de terres présentant un important stock de carbone, cela peut entraîner des émissions notables de gaz à effet de serre ».

66. Particulièrement, la part des biocarburants produits à partir de céréales, plantes sucrières et oléagineuses ou plantes cultivées essentiellement à des fins de production d'énergie, est plafonnée (cons. 17-22, art. 2). 
3) La protection des sols contre la pollution des sols par les boues issues du traitement des eaux usées

L'épandage des résidus issus du traitement des eaux usées, appelés «boues », constitue une solution moins coûteuse que leur incinération. Et dans la mesure où les boues peuvent présenter une qualité agronomique, leur valorisation en agriculture a été encouragée ${ }^{67}$. Néanmoins, parce que l'opération peut présenter des risques de pollution des sols, le milieu agricole a demandé des garanties, apportées par une réglementation des conditions de l'épandage. C'est au demeurant, ainsi que le relève M. Desrousseaux ${ }^{68}$, la première fois que la Commission européenne s'est saisie de la question des sols ${ }^{69}$.

Un principe liminaire est posé : «l'épandage des boues ne peut être pratiqué que si celles-ci présentent un intérêt pour les sols ou pour la nutrition des cultures et des plantations. Il est interdit de pratiquer des épandages à titre de simple décharge $\gg^{70}$. A fortiori, l'épandage ne doitil pas porter atteinte à la qualité des sols ${ }^{71}$ et les risques de contamination par les métaux lourds, présents dans les résidus d'épuration, doivent être prévenus. Pour ce faire, l'épandage est soumis à autorisation administrative préalable ou à déclaration ${ }^{72}$, les conditions de traitement des boues avant épandage, leurs caractéristiques techniques sont réglementées, ainsi que les modalités d'épandage ${ }^{73}$.

\section{4) La protection des sols dans les Alpes}

Le protocole d'application de la Convention alpine ${ }^{74}$ dans le domaine de la protection des sols, adopté le 16 octobre 1998, est un texte tout

67. En ce sens, le $7^{\mathrm{e}}$ considérant de la directive $86-278 \mathrm{du} 12$ juin 1986 relative à la protection de l'environnement et notamment des sols lors de l'utilisation des boues d'épuration en agriculture dispose : « les boues peuvent présenter des propriétés agronomiques utiles et que, par conséquent, il est justifié d'encourager leur valorisation en agriculture ».

68. Thèse précitée, $n^{\circ} 164$.

69. Ainsi que le souligne le texte lui-même, « cette directive vise en outre à établir certaines premières mesures communautaires dans le cadre de la protection des sols ", $6^{\mathrm{e}}$ cons..

70. Art. 6 al. 2 décret n 97-1133 du 8 décembre 1997 relatif à l'épandage des boues issues du traitement des eaux usées.

71. Art. 6 al. 1 décret $n^{\circ}$ 97-1133.

72. Art. R. 214-1 C. env., rubrique 2.1.3.0.

73. Arrêté du 8 janvier 1998 fixant les prescriptions techniques applicables aux épandages de boues sur les sols agricoles pris en application du décret n 97-1133 du 8 décembre 1997 relatif à l'épandage des boues issues du traitement des eaux usées.

74. $C f$. décret $\mathrm{n}^{\circ} 2006-125$ du 31 janvier 2006 portant publication du protocole d'application de la Convention alpine de 1991 dans le domaine de la protection des sols. 
à la fois ambitieux et peu contraignant tant les mesures prescrites aux Etats signataires restent imprécises.

Ambitieux le Protocole l'est dans ses objectifs et la perspective embrassée. Le protocole vise à prévenir tous types d'atteintes aux sols, " atteintes d'ordre quantitatif et qualitatif », érosion, imperméabilisation, pollution, dégradation de la structure des sols; il proclame que la « restauration de la fonction naturelles (des sols est) d'intérêt général » et que " les intérêts économiques devront être harmonisés avec les exigences écologiques ». Pour autant, les dispositions du Protocole n'engagent que les Etats à agir en adoptant les mesures nécessaires à sa mise en oeuvre et sa terminologie révèle combien les prescriptions du texte sont souples ${ }^{75}$. «Vaste catalogue de bonnes intentions ", l'essentiel des dispositions du Protocole n'est donc pas directement applicable ${ }^{76}$.

En conclusion, mêlant incitations et contraintes, obligations de faire et de ne pas faire, le droit tend à prévenir certaines dégradations des sols. Mais l'inventaire de ces normes révèle que cela demeure marginal ; leur champ d'application est restreint par leur objet et/ou leur ressort géographique. C'est donc la politique agricole commune et les aides associées qui comprennent les dispositions les plus ambitieuses en matière de protection des sols ; mais par nature, elles ne visent que les personnes qui ont une activité agricole et plus précisément celles qui sont bénéficiaires d'aides au titre de la politique agricole commune.

\section{5) PAC et sol}

La politique agricole commune a intégré la protection de l'environ-

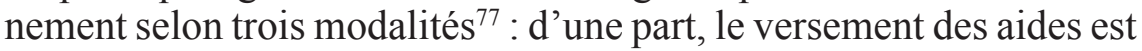

75. Ainsi, « la renaturalisation des sols endommagés est à encourager » (art. 1.2), « les aspects de protection doivent en règle générale primer les aspects d'utilisation » (art. 2.2), « les Parties contractantes examinent les possibilités d'appuyer les mesures visées par le présent protocole... par des mesures fiscales et/ou financières. Les mesures compatibles avec la protection du sol et avec les objectifs d'une utilisation économe et écologique du sol devraient bénéficier d'un soutien particulier» (art 2.3), « les surfaces endommagées par l'érosion du sol et les glissements de terrain devraient être assainis autant que nécessaire » (art. 11.2), etc. 76. Ce qui signifie qu'un requérant ne serait pas fondé à se prévaloir de la violation de ses dispositions à l'occasion d'un litige ; pour les conditions de l'applicabilité directe d'une norme internationale, CE Ass. 11 avril 2012, Gisti, req. ${ }^{\circ} 322326$.

77. Pour plus de développements, cf. Hermon C. «Agriculture et environnement. Un nouveau projet pour la PAC ? », Revue de l'Union Européenne, janvier 2014, n 574, p. 52. 
subordonné au respect de certaines exigences environnementales ${ }^{78}$, d'autre part des aides sont proposées en contrepartie d'engagements agro-environnementaux ${ }^{79}$, enfin les agriculteurs ne bénéficient dorénavant de l'intégralité des paiements directs au titre du premier pilier $^{80}$ que s'ils intègrent dans leur système de production certaines pratiques agronomiques bénéfiques à l'environnement et au climat ${ }^{81}$. Or, la protection des sols fait partie des enjeux environnementaux identifiés par la PAC.

Au titre de la conditionnalité des aides, pour prévenir l'érosion des sols et maintenir un certain niveau de matières organiques dans les sols, les « bonnes conditions agricoles et environnementales $»^{82}$ interdisent : $1^{\circ}$ - de travailler des sols gorgés d'eau ou inondés (art. D. $615-51$ C. rural), $2^{\circ}$ - de labourer les parcelles qui ont une pente supérieure à $10 \%$, entre le $1^{\text {er }}$ décembre et le 15 février, autrement que perpendiculairement à la pente ${ }^{83}, 3^{\circ}$ - de brûler les résidus de cultures (art. D. 615-47 C. rural). Par ailleurs, l'obligation d'une couverture minimale des sols (art. D. 615-50 C. rural) participe également à leur protection, même si la mesure est dite poursuivre en premier lieu l'objectif de stockage du carbone.

Au titre du second pilier de la PAC, certains contrats agroenvironnementaux peuvent inciter à adopter des pratiques de nature à protéger la qualité des sols. Ainsi, une « mesure agroenvironnementale rotationnelle » a été proposée aux agriculteurs de 2007 à $2010^{84}$. Surtout, une mesure « conversion au semis direct sous couvert » vient d'être ouverte par le ministère de l'agriculture. Selon la fiche de présentation du ministère, datée du 21 avril $2017^{85}$, la mesure permet « de répondre aux enjeux liés à une gestion pérenne des sols agricoles : l'érosion, la matière organique, l'activité

\footnotetext{
78. Conformément au principe dit de la conditionnalité des aides.

79. Constitutifs du $2^{\text {nd }}$ pilier de la PAC.

80. Constitué de mesures de soutien des marchés et d'aides aux revenus des agriculteurs.

81. Art. D. 615-31 et ss. C. rural.

82. Les « bonnes conditions agricoles et environnementales », définies en partie par les Etats membres, complètent les exigences réglementaires européennes, pour constituer les règles de la conditionnalité. Art. 93 règlement n 1306/2013 du 17 décembre 2013 relatif au financement, à la gestion et au suivi de la politique agricole commune.

83. Sauf si une bande végétalisée pérenne d'au moins 5 mètres est implantée en bas de la parcelle, art. D. 615-51 C. rural.

84. Rapport annuel d'exécution du PDRH pour l'année 2012, p. 53

85. Nous remercions Monsieur Fauré, Chef du Bureau Aides aux Zones Défavorisées et à l'Agro-environnement au ministère de l'agriculture, de nous avoir adressé ce texte.
} 
biologique et le tassement. ... (Elle) incite les exploitants à limiter au maximum leur travail du sol, à mettre en place un couvert tout au long de l'année et à diversifier les rotations culturales sur les terres de grandes cultures. Le travail mécanique des sols est remplacé par le travail des organismes du sol (travail biologique) et le travail du système racinaire des végétaux... (Elle) promeut la pratique du semis direct sous couvert végétal » sans travail du sol préalable. Par le contrat, d'une durée de cinq ans, l'agriculteur s'engage à convertir à l'agriculture de conservation la première année au moins $40 \%$ de ses surfaces bénéficiant de l'aide, la seconde année au moins $60 \%$, la troisième année au moins $80 \%$, puis la totalité de la surface ${ }^{86}$. La mesure agro-environnementale " systèmes grandes cultures " soutient également des pratiques bénéfiques à la qualité du sol. Outre la diminution des herbicides et l'introduction obligatoire d'une part de légumineuses, la mesure subordonne les aides à l'introduction d'une diversification et d'une rotation des cultures.

Enfin, les nouvelles conditions environnementales du premier pilier viennent conforter le dispositif en prescrivant des mesures simples. Pour bénéficier du complément au paiement de base, les agriculteurs doivent : diversifier leurs cultures ${ }^{87}$, conserver leurs prairies permanentes et maintenir ou créer des " surfaces d'intérêt écologique », telles que des haies, arbres, bandes enherbées, surfaces en jachère, en agroforesterie...

L'ensemble constitue certainement un levier à une meilleure prise en compte par les agriculteurs de la qualité et de la santé de leurs sols. La conditionnalité des aides et le complément au paiement de base invitent, sous peine de réduction des aides, à adopter des pratiques élémentaires pour les sols et les nouvelles mesures agro-environnementales visées ci-dessus peuvent conduire à une

\footnotetext{
86. L'aide est de 163,79 euros par hectare engagé par an. L'agriculteur doit par ailleurs convertir au moins la moitié de la surface de son exploitation, et la surface engagée ne doit pas être inférieure à 10 hectares. Au surplus, sur l'ensemble de l'exploitation, y compris les parcelles non converties, l'agriculteur ne doit pas dépasser le seuil de traitement propre au territoire (Indice de Fréquence des Traitements herbicides et hors herbicides) et sur les parcelles bénéficiant de l'aide, il doit respecter plusieurs engagements : analyse des sols, enregistrement des pratiques, bilans humiques, suivi de l'indicateur vers de terre de l'observation agricole de la biodiversité, diversification et rotation des cultures.

87. Au moins deux cultures lorsque la surface exploitable dépasse 10 hectares, au moins trois lorsqu'elle dépasse trente hectares.
} 
transition vers des techniques de production (plus ${ }^{88}$ ) respectueuses des sols. Mais, en droit, rien n'oblige, bien entendu, l'agriculteur à souscrire une MAEC, ni même d'ailleurs à respecter les conditions posées au versement intégral des aides ${ }^{89}$. Au demeurant, si les différentes réformes ont contribué à une plus grande considération de l'environnement, la PAC n'a pas mené sa révolution écologique et son objectif prioritaire reste la production ${ }^{90}$.

A défaut de prévention de la dégradation des sols, le droit comprend des obligations de restauration. Leur mise en œuvre reste toutefois aujourd'hui cantonnée aux anciens sites industriels lorsqu'il s'agit d'en modifier l'usage.

\section{B. LA RESTAURATION DES SOLS}

En droit, la restauration des sols peut être imposée sur le fondement de la directive " responsabilité environnementale ${ }^{91}$ (1) ou du droit des installations classées (2). Au surplus, la loi n 2014-366 du 24 mars 2014 ajoute un nouveau chapitre dans le Code de l'environnement intitulé « sites et sols pollués ", la question restant posée de savoir si ces dispositions créent, ou pas, de nouvelles obligations de remise en état (3). Le droit des installations classées et la loi n 2014-366 envisagent la remise en état à des fins d'urbanisation des anciens sites industriels dont l'activité a laissé subsister des pollutions dans le sol. Quant à la directive « responsabilité environnementale », elle n'a jamais été mise en œuvre, ce qui certes n'interdit pas une exécution future mais laisse comprendre que ses conditions d'application et le régime institué constituent des freins à son déploiement ${ }^{92}$. Ainsi, la restauration des sols en droit est étroitement circonscrite aux

88. La mesure « systèmes grandes cultures » n'a pas pour cible première les sols, mais la biodiversité et la qualité de l'eau; elle devrait toutefois permettre de limiter l'impact de ces systèmes de culture en diminuant les transferts de polluants et en améliorant la fixation de l'azote.

89. Etant entendu qu'il peut en revanche être difficile économiquement de supporter une baisse des aides.

90. Pour plus de développements, $c f$. Hermon C. «Agriculture et environnement. Un nouveau projet pour la PAC ? », précité.

91. Directive 2004/35/CE du 21 avril 2004 sur la responsabilité environnementale en ce qui concerne la prévention et la réparation des dommages environnementaux.

92. Cf. Hermon C. « La réparation du dommage écologique. Les perspectives ouvertes par la directive du 21 avril 2004 », AJDA 2004, p. 1792, Kromarek P. et Jacqueau M. « Réflexions autour de la transposition de la directive sur la responsabilité environnementale en droit français ", Env. et $D D$ nov. 2004, n 11, Etude 18. 
hypothèses de pollution d'une part, à des fins d'urbanisation d'autre part ; singulièrement, la probabilité d'une utilisation de ces textes à des fins de restauration de sols agricoles dégradés ${ }^{93}$ est faible.

1) La directive « responsabilité environnementale » et la réparation des dommages au sol

La directive sur la responsabilité environnementale (DRE) ${ }^{94}$ institue une forme de réparation de certains dommages écologiques, inédite jusqu'alors, à la charge de l'exploitant de l'installation responsable du dommage. Son absence d'application à ce jour nous conduit toutefois à rester brève.

Une première lecture de la DRE peut laisser croire que les dégradations des sols pourraient trouver une résolution sur son fondement. Toutes les atteintes à l'environnement ne relèvent pas de la directive, mais les dommages affectant les sols sont visés expressément (art. 2.1) ${ }^{95}$. $\mathrm{Au}$ surplus, le dommage est entendu comme l'atteinte à la ressource ou à un service rendu (art. 2.2 $)^{96}$. Mais, en seconde lecture, il apparaît que la définition du dommage est très restreinte puisque ne constitue un dommage au sol réparable que la « contamination des sols qui engendre un risque d'incidence négative grave sur la santé humaine du fait de l'introduction directe ou indirecte en surface ou dans le sol de substances, préparations, organismes ou micro-organismes $\gg$. En conséquence, les mesures de réparation sont conduites au regard de ce seul enjeu sanitaire : « les mesures nécessaires sont prises afin de garantir au minimum la suppression, le contrôle, l'endiguement ou la réduction des contaminants concernés, de manière à ce que les sols contaminés, compte tenu de leur utilisation actuelle ou prévue

93. Par exemple en prescrivant l'implantation de haies pour lutter contre l'érosion des sols ou l'implantation de cultures intermédiaires pour résorber l'excès de nitrate.

94. Directive 2004/35/CE du 21 avril 2004, transposée par la loi nº 2008-757 du $1^{\text {er }}$ août 2008, codifiée sous les articles L. 162-1 et ss. du Code de l'environnement.

95. Etant toutefois exclues les pollutions diffuses, ce qui limite grandement les possibilités d'application de la directive en cas de dommage aux sols, « sauf si un lien de causalité entre les dommages ou leur menace et les activités des différents exploitants est établi » art. L. $161-2-7^{\circ}$ C. env..

96. Constitue un dommage « une modification négative mesurable d'une ressource naturelle ou une détérioration mesurable d'un service lié à des ressources naturelles, qui peut survenir de manière directe ou indirecte ". Pour une analyse de cette extension du dommage à l'atteinte aux services, cf. I. Doussan « Les services écologiques : un nouveau concept pour le droit de l'environnement ", in Cans C. (dir.), La responsabilité environnementale, Dalloz, 2009, p. 125. 
pour l'avenir..., ne présentent plus de risque grave d'incidence négative sur la santé humaine » (annexe II.2). L'approche est donc extrêmement réductrice. Elle se révèle finalement proche de celle en vigueur en droit des installations classées.

2) Le droit des installations classées et la remise en état des sols pollués

Le droit des installations classées astreint le dernier exploitant de l'installation, lorsqu'il cesse son activité, à mettre son site dans un état tel qu'il ne s'y manifeste aucun danger ni inconvénient pour les riverains et pour l'environnement. Ceci peut exiger un démantèlement des bâtiments, l'évacuation de matières dangereuses, mais également la décontamination de sols pollués. L'étendue de la remise en état exigible à ce titre a été précisée : le site doit être remis dans un état tel qu'il puisse permettre un usage futur du site déterminé conjointement avec le maire ou le président de l'EPCI ${ }^{97}$ compétent en matière d'urbanisme et le propriétaire du site s'il n'est pas lui-même l'exploitant ${ }^{98}$. La remise en état des sols pollués est ainsi envisagée en considération de l'usage à venir du site, et particulièrement en considération d'un potentiel usage résidentiel ${ }^{99}$ dans un contexte où l'on entend, pour « construire la ville sur la ville » comme vu supra, reconvertir les friches industrielles. Elle n'est pas conduite plus globalement au regard d'un objectif de restauration de la qualité ou de la santé du sol contaminé ${ }^{100}$.

On peut toutefois relever le régime particulier réservé à la remise en état des carrières ${ }^{101}$ pour laquelle une attention est portée aux sols

97. Etablissement public de coopération intercommunale.

98. Art. L. 512-6-1 et L. 512-7-6 C. env.

99. En l'absence d'accord entre l'exploitant, le propriétaire et le maire (ou le président de l'EPCI), la remise en état, prescrite par le préfet, doit permettre au moins « un usage futur du site comparable à celui de la dernière période d'exploitation de l'installation mise à l'arrêt ». Mais «dans le cas où (une telle) réhabilitation... est manifestement incompatible avec l'usage futur de la zone... le préfet peut fixer ... des prescriptions de réhabilitation plus contraignantes permettant un usage du site cohérent avec les documents d'urbanisme " (art. L. 512-6-1 et L. 512-7-6 C. env.).

100. Sur le fait que la remise en état des sols pollués est envisagée, en droit, à la hauteur de seuils de toxicité et au regard des usages réservés au site, sans obligation de restauration de la qualité des sols, cf. Desrousseaux M., thèse précitée, n 622 et 663 et ss..

101. Etant rappelé que les carrières constituent des installations classées au-delà de certaines surfaces d'exploitation ou quantité de matériaux à extraire, $c f$. Nomenclature des installations classées, rubrique 2510 . 
en tant que tels : « le remblayage des carrières est géré de manière à assurer la stabilité physique des terrains remblayés. Il ne nuit pas à la qualité du sol... ${ }^{102}$.

\section{3) La loi ALUR et la remise en état des sols pollués}

L'article 173 de la loi ALUR ${ }^{103}$ ajoute un nouvel article dans le Code de l'environnement sous un chapitre intitulé « Sites et sols pollués ». Le contexte dans lequel s'inscrit cette disposition et l'intention des auteurs de la loi «pour l'accès au logement et un urbanisme rénové » sont les mêmes que ceux décrits ci-dessus : il s'agit de valoriser les friches industrielles là où le foncier urbanisable manque. Mais si l'on s'en tient aux termes de la loi, sa portée est plus difficile à mesurer : simple clarification du droit quant aux personnes tenues de financer les actions de dépollution ou progression des obligations de remise en état?

Selon l'article L. 556-3-I du code, « en cas de pollution des sols ou de risques de pollution des sols présentant des risques pour la santé, la sécurité, la salubrité publiques et l'environnement au regard de l'usage pris en compte, l'autorité titulaire du pouvoir de police peut, après mise en demeure, assurer d'office l'exécution des travaux nécessaires aux frais du responsable ». Au regard du droit des installations classées, cette disposition ne crée pas de nouvelles obligations sauf si elle est applicable alors même qu'il n'y a pas cessation d'activité. Le texte ne précise rien à ce propos et il appartiendra au juge de trancher soit en raisonnant par analogie avec le droit des installations classées, soit en s'en tenant à une lecture littérale du texte selon laquelle il y a remise en état « en cas de pollution des sols... présentant des risques » au regard de l'usage du sol, et alors même qu'il n'y aurait ni changement d'usage, ni cessation d'activité.

Par ailleurs, le champ d'application de l'article L. 556-3-I du Code de l'environnement dépasse celui des installations classées. En effet, le paragraphe II de l'article L. 556-3 énumérant les personnes tenues à l'obligation de remise en état vise les sols pollués par des déchets, quelle qu'en soit l'origine, y compris donc, non issus

102. Art. 12.3 arrêté 22 sep. 1994 relatif aux exploitations de carrières et aux installations de premier traitement des matériaux de carrières, modifié par arrêté du 5 mai 2010, cf. Desrousseaux M., thèse précitée, $\mathrm{n}^{\circ}$ 688-689.

103. Loi n 2014-366 du 24 mars 2014 pour l'accès au logement et un urbanisme rénové. 
d'une installation classée. Or, si la remise en état des sols pollués sur le fondement du droit des déchets a été admise dans un premier temps, les sols pollués étant assimilés à des déchets ${ }^{104}$, elle ne l'est plus depuis l'entrée en vigueur de la directive $n^{\circ}$ 2008/98/CE du 19 novembre 2008 relative aux déchets ${ }^{105}$. Dès lors, 1'article L. 556-3-I du Code de l'environnement semble constituer une progression des obligations de remise en état des sols pollués par des déchets, inexistantes en l'état du droit. Ainsi pourrait-on envisager notamment que des remises en état des sols agricoles pollués par des matières fertilisantes d'origine résiduaire ${ }^{106}$, considérés comme des déchets ${ }^{107}$, puissent être ordonnées ${ }^{108}$. Le raisonnement pourrait être repris à propos des sols pollués par les reliquats de produits phytosanitaires $^{109}$ qui n'ont pas atteint leur cible, constitutifs de déchets ${ }^{110}$. Si tel devait être la lecture du texte, alors sa portée pour la restauration des sols agricoles serait considérable... à supposer que quelqu'un y ayant intérêt s'en saisisse et demande à ce qu'il soit appliqué en ce sens.

In fine, si le droit positif n'est pas vide de dispositions relatives à la protection des sols, la juxtaposition de l'ensemble de ces textes ne garantit assurément pas une protection complète des sols en général, des sols agricoles en particulier; leur champ d'application ou leur objet est trop restreint. En ce sens, la Commission européenne, dans deux communications successives, a conclu : « même si plusieurs

104. CJCE 7 septembre 2004 SA Texaco Belgium, CAA Versailles 10 mai 2007, req. $\mathrm{n}^{\circ} 05 \mathrm{VE} 01492$.

105. La directive exclut de son champ d'application « les sols in situ y compris les sols pollués non excavés ».

106. Pour plus de développements, cf. Bellec P., Lavarde P., Lefèbvre L. et Madignier M.-L., Propositions pour un cadre national de gestion durable des sols, précité, pp. 45-47.

107. Selon l'article L. 541-1-1 du Code de l'environnement, " au sens du présent chapitre (Prévention et gestion des déchets), on entend par "Déchet" toute substance ou tout objet, ou plus généralement tout bien meuble, dont le détenteur se défait ou dont il a l'intention ou l'obligation de se défaire ». Pour plus de développements sur la qualification de « déchet» des matières fertilisantes, cf. Langlais A., « Le droit face à la gestion des effluents et des émissions d'azote ", in Les flux d'azote liés aux élevages. Réduire les pertes, rétablir les équilibres, Expertise collective, rapport, INRA, 2012, p. 429 et ss..

108. Cette remise en état pourrait être ordonnée : au producteur des déchets, à l'agriculteur en qualité de détenteur desdites matières s'il a commis une faute ou, à défaut, au propriétaire de l'assise foncière « s'il a fait preuve de négligence ou (s'il) n'est pas étranger à cette pollution » (art. L. 556-3-II C. env.).

109. Cf. Bellec P., Lavarde P., Lefèbvre L. et Madignier M.-L., Propositions pour un cadre national de gestion durable des sols, précité, p. 45.

110. L'agriculteur serait ici également responsable en qualité de producteur de déchets. 
politiques existantes assurent une protection des sols, il n'existe pas d'approche... cohérente et complète de la protection des sols. La protection des sols est davantage le résultat de la nature transversale du sol (bénéficiant ainsi d'une législation qui ne le vise pas directement) que d'une intention explicite de traiter les problèmes des sols $»^{111}$. Il en est ressorti une proposition de directive définissant un cadre pour la protection des sols, « destinée à combler cette lacune $»^{112}$, qui aurait pu constituer le socle d'un « droit du sol» si ladite proposition avait été maintenue.

\section{VERS LA CONSTRUCTION D’UN « DROIT DU SOL »?}

La proposition de directive, déposée par la Commission en 2006, n'a pas été adoptée (A). Néanmoins, les réflexions convergent : un constat de la dégradation des sols est dressé ${ }^{113}$, ceci est en partie lié aux insuffisances de l'encadrement juridique, et un appel à la protection par le droit est lancé, appel fondé sur les fonctions du sol et les services rendus par les sols (B).

\section{A. LA PROPOSITION DE DIRECTIVE SUR LES SOLS}

La proposition de directive définissant un cadre pour la protection des sols ${ }^{114}$ était remarquable à trois titres : elle identifiait clairement

\footnotetext{
111. Communications Commission européenne : Vers une stratégie thématique pour la protection des sols, 16 avril 2002, COM(2002)179final, vue infra, également en ce sens : Stratégie thématique en faveur de la protection des sols, 22 sep. 2006, COM(2006)231, vue infra et, plus récemment, Conseil de l'environnement, 3 mars 2014, cité in Desrousseaux M., thèses précitée, p. 393, relevant « le manque de cohérence » de la protection actuelle dû à une prise en compte « sectorielle» : « l'approche actuelle, combinée avec des législations nationales majoritairement limitées à la question des sites et sols pollués, n'a pas endigué la dégradation des sols au sein de l'Union européenne ».

112. Exposé des motifs, projet de texte. Dans son exposé des motifs à la proposition de directive, la Commission relevait : " étant donné la diversité de leurs objectifs et de leur champ d'application, et parce qu'elles visent souvent à préserver d'autres milieux naturels, ces dispositions existantes, quand bien même pleinement mises en œuvre, n'assurent qu'une protection fragmentaire des sols puisqu'elles ne concernent pas tous les sols ni toutes les menaces recensées. En conséquence, la dégradation des sols continue ».

113. Bellec P., Lavarde P., Lefèbvre L. et Madignier M.-L., Propositions pour un cadre national de gestion durable des sols, précité, p. 30 et ss, FAO and ITPS, Status of the World's Soil Resources (SWSR)- Technical Summary, Rome, 2015, Bispo A., Guellier C., Martin E., Sapijanskas J., Soubelet H. et Chenu C. (coord.) Les sols. Intégrer leur multifonctionnalité pour une gestion durable, précité, pp. 32 et ss..

114. Proposition de directive définissant un cadre pour la protection des sols et modifiant la directive 2004/35/CE, 22 sep. 2006, COM(2006)232final.
} 
ce qui était objet de la protection ${ }^{115}$, elle incluait dans cet objet les fonctions du sol et services qui en résultent ${ }^{116}$, et adoptait une approche exhaustive de ces fonctions et services.

Selon l'exposé des motifs du projet de texte, « les dispositions législatives proposées... visent à protéger les sols et à préserver leur aptitude à remplir leurs fonctions écologiques, économiques, sociales et culturelles ». Ceci était précisé dans l'article $1^{\text {er }}$ de la proposition : « la présente directive définit un cadre pour la protection des sols et la préservation de leur capacité à remplir chacune des fonctions écologiques, économiques, sociales et culturelles suivantes : a) production de biomasse, notamment pour l'agriculture et la foresterie ; b) stockage, filtrage et transformation d'éléments nutritifs, de substances et d'eau ; c) vivier de la biodiversité, notamment habitats, espèces et gènes $d$ ) environnement physique et culturel de l'homme et des activités humaines; e) source de matières premières ; f) réservoir de carbone; g) conservation du patrimoine géologique et architectural ».

Cette approche systémique, incluant les fonctions et services du sol, constituait l'apport essentiel du texte ${ }^{117}$. Le reste en découlait, « la directive prévoit des mesures pour prévenir les processus de dégradation des sols, tant naturels que provoqués par les activités humaines, qui compromettent la capacité des sols à remplir ces fonctions » $\left(\right.$ art. $\left.1^{\text {er }}\right)$. A cette fin, la proposition comprenait une obligation d'évaluer les impacts sur les sols de la conduite de certaines politiques sectorielles ${ }^{118}$ et de recenser les « zones dans lesquelles il est patent ou hautement probable qu'un ou plusieurs processus de

115. Elle répondait à ce titre à l'observation de Desrousseaux M., thèse précitée, $n^{\circ} 610$ : « Nul doute que les faiblesses du droit à lutter efficacement contre la dégradation des sols... soient une conséquence des difficultés du droit à identifier l'objet réel de la protection ».

116. La proposition de texte confond fonction et service ; elle ne vise expressément que les «fonctions » du sol sans distinguer lesdites fonctions (de séquestration du carbone, vivier de biodiversité ou de stockage d'eau et d'éléments nutritifs), des services, services culturels ou services de production de matières premières, de biomasse pour l'agriculture et la foresterie, visés dans le texte.

117. Sur le changement d'approche méthodologique que suppose l'appréhension par les fonctions et services, $c f$. Fèvre M. «Les "services écosystémiques", une notion fonctionnelle », infra.

118. « Lors de l'élaboration de politiques sectorielles susceptibles d'aggraver ou d'atténuer les processus de dégradation des sols, les Etats... évaluent les incidences de ces politiques sur ces processus, en particulier dans les domaines ... de l'agriculture... du changement climatique, de l'environnement, de la protection de la nature et des paysages ", art. 3 . 
dégradation (érosion, diminution des teneurs en matières organiques, tassement, salinisation, glissement de terrains) s'est produit ou risque de se produire dans un avenir proche » (art. 6). De plus, les Etats membres devaient adopter des objectifs de réduction des risques d'atteinte aux sols, sans toutefois que des normes de qualité soient posées par la directive, ainsi que des programmes de mesures pour atteindre ces objectifs ${ }^{119}$, comprenant spécifiquement des mesures visant à limiter " l'introduction de substances dangereuses dans le sol afin d'éviter la contamination de ce dernier et d'en préserver les fonctions » (cons. 20, art. 9). Les Etats devaient enfin conduire les utilisateurs des terres à prendre « des précautions lorsqu'il est probable que l'usage qu'ils font du sol compromettra sensiblement les fonctions de ce dernier $»^{120}$.

La proposition de directive, déposée sur le fondement de l'article $175 \S 1$ du traité $C E^{121}$, devait recueillir une majorité qualifiée devant le Conseil. Elle a achoppé sur la réticence des Etats, inquiets « des enjeux importants (soulevés par ce projet de directive) tant en matière de politique industrielle qu'au regard de l'activité agricole $\rangle^{122}$ et du coût induit par la mise en œuvre des mesures envisagées, particulièrement pour répondre à la contamination des sols $\mathrm{s}^{123}$. La proposition a donc été abandonnée en 2014. Elle aurait pu revenir dans l'agenda législatif. Une initiative citoyenne européenne a été lancée en septembre 2016, en application de l'article 11.4 du Traité sur l'Union Européenne, demandant l'adoption d'une « législation spécifique en matière de protection du sol $»^{124}$. Mais la pétition n'a

119. Art. 8.1, Programmes de mesures destinées à lutter contre l'érosion, la diminution des teneurs en matières organiques, le tassement, la salinisation et les glissements de terrain. «Afin de préserver les fonctions des sols ..., les États membres établissent, au niveau approprié, pour les zones à risques..., un programme de mesures comprenant au moins des objectifs de réduction des risques, les mesures appropriées pour atteindre ces objectifs, un calendrier de mise en oeuvre de ces mesures et une estimation des fonds publics ou privés nécessaires pour les financer $»$.

120. Cons. 12, en ce sens également, art. 4.

121. «Le Conseil... décide des actions à entreprendre par la Communauté en vue de réaliser les objectifs visés à l'article 174 » relatif à l'environnement.

122. Raoult P., Rapport Sénat au nom de la Commission des Affaires économiques sur la proposition de résolution... sur la proposition de directive, doc. Sénat, $\mathrm{n}^{\circ} 290,17$ avril 2007, p. 7.

123. En ce sens, délégation pour l'Union européenne, Sénat, 9 nov. 2006 : « la délégation pour l'Union européenne du Sénat... demande à la Commission européenne de garantir la proportionnalité des mesures envisagées, et notamment de justifier que le coût de chacune de celles-ci est raisonnable au vu des avantages attendus »

124. https://www.people4soil.eu.fr 
pas recueilli le nombre de signatures requis pour que la Commission s'en saisisse ${ }^{125}$. Quoi qu'il en soit, cette initiative citoyenne n'est pas isolée ; les appels à la construction d'un droit du sol sont nombreux.

\section{B. LES APPELS À LA CONSTRUCTION D’UN DROIT DU SOL}

Les appels à la protection des sols sont anciens et récurrents. Dès 1972, le Conseil de l'Europe invitait à l'adoption d'une « législation appropriée... (pour) protéger les sols... et au besoin les restaurer » en relevant notamment, s'agissant des sols agricoles, « la destruction des matières organiques du sol (et de sa structure) par des pratiques agricoles inadéquates et le mauvais emploi d'engins lourds », ainsi que « la pollution du sol» par « certains engrais chimiques et pesticides... utilisés sans discernement $»{ }^{126}$. Dans une perspective strictement alimentaire, la FAO en 1981 adoptait également une « Charte mondiale des sols » où elle engageait les Etats à « incorporer dans la législation concernant les ressources, des principes rationnels d'utilisation des terres, d'aménagement et de conservation des sols ».

Aujourd'hui, l'ONU exhorte la communauté internationale à se doter d'un protocole additionnel à la Convention de lutte contre la désertification en s'appuyant sur le concept de Soil Security, « sécurité des sols ", transcendant la seule question de la désertification ${ }^{127}$, et, parmi ses objectifs de développement durable, a retenu le principe de neutralité en matière de dégradation des terres (NDT) ${ }^{128}$. En 2010, le Comité de l'agriculture de la FAO s'est de nouveau emparé de la question des sols et a invité l'agence de l'ONU à renouveler son

\footnotetext{
125. Selon le règlement $n^{\circ}$ 211/2011 du 16 février 2011 relatif à l'initiative citoyenne, la pétition aurait dû recueillir un million de signatures de ressortissants de sept Etats membres dans un délai d'un an pour que la Commission soit saisie de la demande, tout en restant libre de la réponse à y apporter.

126. Charte européenne des sols, RJE 1976, n 3-4, p. 421.

127. Concept développé lors de la journée mondiale de lutte contre la désertification en 2009 ; pour plus de développements, cf. Desrousseaux M., thèse précitée, n 566 et ss..

128. Résolution de l'Assemblée générale « Transformer notre monde : le Programme de développement durable à l'horizon 2030 », 25 sep. 2015, A/RES/70/1, Objectif de développement durable $\mathrm{n}^{\circ} 15$, cible 15.3 : « d'ici à $2030, \ldots$ restaurer les terres et sols dégradés... et s'efforcer de parvenir à un monde sans dégradation des terres ». Cette neutralité en matière de dégradation des terres est définie comme « un état dans lequel la quantité et la qualité des ressources en terre nécessaires pour soutenir les fonctions et services écosystémiques et améliorer la sécurité alimentaire restent stables ou augmentent à des échelles temporelles et spatiales et des écosystèmes donnés », $c f$. " Neutralité en matière de dégradation des terres (NDT) : programme d'appui à la définition des cibles », UNCCD, 2016.
} 
« attention aux sols », " ressource non renouvelable » et « fournisseur de services écosystémiques vitaux $»{ }^{129}$. Il en a résulté une « Charte mondiale des sols révisée » adoptée en juin 2015, élargissant la perspective aux fonctions de régulation de l'eau et de concentration des gaz à effet de serre dans l'atmosphère $(\$ 5)$, à leur qualité de "réservoir essentiel de la diversité biologique mondiale, micro-organismes aussi bien que flore et faune » $(\S 8)$, et aux services associés. Les rapports, communications et avis sur les sols se multiplient, issus tant des instances internationales et européennes que nationales. La convergence de ces textes est frappante : d'une part ils en appellent au droit, à l'adoption d'une législation spécifique, d'autre part ils s'appuient très largement sur les fonctions et services rendus par les sols pour justifier la nécessité d'un renouvellement de la protection ${ }^{130}$. On ne s'en étonnera pas; la référence aux fonctions et aux services est dorénavant récurrente ${ }^{131}$.

\section{La doctrine relaie le propos. Ainsi selon Maylis Desrousseaux : « pour mettre l'accent sur la préservation, la qualité des sols via les services peut permettre la reconnaissance et la protection de leur valeur environnementale », " la notion de qualité, qui recouvrirait l'ensemble des services que rendent les sols... pourrait s'avérer pertinente pour élaborer un principe de protection des sols $\gg{ }^{132}$.}

129. FAO, Comité de l'agriculture, Le sol : sécurité alimentaire, adaptation au changement climatique et atténuation du changement, $22^{\mathrm{e}}$ session, 16-19 juin 2010, COAG/2010/4.

130. En ce sens, Communications de la Commission européenne, "Vers une stratégie thématique pour la protection des sols, Stratégie thématique en faveur de la protection des sols, précité, relevant, respectivement, le « large éventail des fonctions essentielles du sol », «l'ensemble des fonctions que peuvent remplir les sols » pour souligner la nécessité de garantir la protection des sols ; Courtoux A. et Claveirole C. " La bonne gestion des sols agricoles : un enjeu de société », Avis du Conseil économique, social et environnemental, mai 2015 : le CESE adresse un appel à la protection du sol « largement méconnu alors qu'il abrite la part la plus importante de la biodiversité de la planète et que les services écosystémiques qu'il rend sont primordiaux »; rapport Bellec P., Lavarde P., Lefèbvre L. et Madignier M.-L., Propositions pour un cadre national de gestion durable des sols, précité : le rapport s'appuie sur les fonctions et services rendus par le sol, dont l'intérêt général est reconnu, pour justifier la mise en œuvre d'une « stratégie nationale sur les sols » (p. 69), à défaut d'une loi (p. 66), et renforcer la cohérence des législations et politiques sectorielles. 131. Cf. infra Doussan I., "Brève histoire de l'intégration de la notion de service écosystémique en droit », Fèvre M., thèse précitée.

132. Respectivement $n^{\circ} 62$ et introduction. La thèse de M. Desrousseaux porte sur la qualité des sols, et non sur les services rendus par les sols, mais elle s'appuie sur la notion de service pour construire le concept de qualité qu'elle défend. Voir également, Langlais A. «L'appréhension juridique de la qualité des sols agricoles par le prisme des services écosystémiques », Dr. rural, $\mathrm{n}^{\circ}$ 435, août 2015, Etude $20:$ " au regard du besoin urgent de considérer les sols, le recours à la notion particulièrement médiatisée de SE peut s'avérer en 
Les notions de fonction et service permettraient donc d'identifier plus clairement ce qui devrait être objet de la protection, tout en renouvelant 1 'approche de cet objet ${ }^{133}$, puis de justifier cette protection et de la rendre acceptable ${ }^{134}$. Mais au-delà de cette fonction identificatrice et légitimante, il s'agit de repérer si une approche expresse ${ }^{135}$ par service peut, en droit, asseoir une protection des sols inexistante jusqu'alors.

La modification de l'article L. 110-1 du Code de l'environnement par la loi « biodiversité » de 2016 et l'intégration subséquente des services écosystémiques parmi les composantes de l'intérêt général ${ }^{136}$ peuvent justifier l'institution d'une protection, y compris en apportant des restrictions aux droits des propriétaires ou des exploitants. Aux termes d'une jurisprudence constante, des atteintes peuvent en effet être portées au droit de propriété ou à la liberté d'entreprendre pour des motifs d'intérêt général, sous réserve qu'elles ne soient pas disproportionnées. Or, la protection de l'environnement constitue aujourd'hui un de ces motifs justifiant les atteintes au droit de propriété ou à la liberté d'entreprendre ${ }^{137}$. Demain, plus spécialement, la protection des services écosystémiques, d'intérêt

soi justifié mais également stratégique pour attirer l'attention sur le besoin de conserver et retrouver des sols de qualité ».

133. Cf. infra Fèvre M. « Les "services écosystémiques", une notion fonctionnelle ».

134. $C f$. en ce sens, Fèvre M., thèse précitée, p. 18 : « les services écosystémiques... (dévoilent) qu'au-delà de la perte en soi que constitue la dégradation d'une ressource naturelle, d'importantes conséquences sur l'économie et le développement humain en découlent », Bellec P., Lavarde P., Lefèbvre L. et Madignier M.-L., rapport précité, p. 4 : «si la notion de "patrimoine commun » fait débat, l'intérêt général des services écosystémiques rendus par le sol est reconnu ».

135. Fèvre M., thèse précitée, p. 70 : « le concept de "services" nous amène à constater combien les approches fonctionnelles et systémiques sont déjà à l'œuvre, mais de façon éclatée, non structurée, dans des législations qui se préoccupent d'objets bien identifiés de l'environnement ». La première partie de la thèse est consacrée à ce repérage des " services écologiques... déjà saisis par le droit ».

136. Aux termes de l'article L. 110-II du Code, modifié par la loi n 2016-1087 du 8 août 2016 pour la reconquête de la biodiversité, de la nature et des paysages, "la sauvegarde des services (fournis par les espaces, ressources et milieux naturels terrestres et marins, les sites, les paysages diurnes et nocturnes, la qualité de l'air, les êtres vivants et la biodiversité, est) d'intérêt général ».

137. $C f$. pour ex. CAA Nantes 31 décembre 2009, Mme Marie Scoarnec, RJE 1-2011, p. 127, concl. Degommier S., Cons. Constit. 11 octobre 2013, n 2013-346 QPC, CE 17 juillet 2017, req. $\mathrm{n}^{\circ}$ 410989. Pour plus de développements, cf. Tomadini A., La liberté d'entreprendre et la protection de l'environnement. Contribution à l'étude des mécanismes de conciliation, LGDJ, 2016. 
général, pourra également être invoquée ${ }^{138}$. Cette approche par les services permettra donc le cas échéant que soient institués des dispositifs de protection quels qu'ils soient, expropriation, servitude d'utilité publique, autorisation préalable, conditions d'exploitation etc, au regard des services particuliers fournis par telles composantes de l'environnement ${ }^{139}$. Pour autant, sans même considérer les obstacles sociaux-économiques ${ }^{140}$, tous les services ne pourront assurément être ainsi protégés, l'exigence de la proportionnalité, telle qu'entendue par la jurisprudence, s'y opposant. Aussi la voie de la valorisation des services et de l'incitation à les préserver nous a-t-elle paru fructueuse, justifiant les développements axés sur ces moyens de valorisation, financiers ${ }^{141}$ et contractuels ${ }^{142}$. Et à défaut de convaincre, l'intégration des atteintes aux services parmi les chefs de préjudices réparables peut dorénavant permettre de réparer ce qui ne l'était pas ${ }^{143}$.

138. Les services fournis par les sols ne sont pas expressément visés par l'article L. 110-II (cf. supra note 132). Nous n'y voyons pourtant pas d'obstacle à la protection de ces services au titre de l'intérêt général dans la mesure où le lien entre les sols et les milieux et ressources énumérés par ledit article est expressément visé dans l'alinéa suivant : « ... les sols... concourent à la constitution du patrimoine » commun de la nation, lui-même constitué par l'ensemble des composantes de l'environnement énuméré par l'article L. 110-II.

139. $C f$. infra Beaussonie G. « La qualification juridique des services écosystémiques ».

140. Les résistances du milieu agricole notamment, seraient fortes. On relèvera en ce sens la position, retranscrite dans le rapport précité, Propositions pour un cadre national de gestion durable des sols : « Tout en soulignant l'impact environnemental positif de leurs pratiques, les agriculteurs les plus engagés souhaitent néanmoins conserver la maîtrise de leur « capital sol »... D'une façon générale, les agriculteurs craignent la mise en place de contraintes particulières en termes d'usages des sols agricoles, à travers notamment des obligations de moyens uniformes, qui pourraient découler d'une volonté d'assujettir leur activité au maintien de services écosystémiques, alors que la question de la reconnaissance des services environnementaux et celle de leur valorisation économique restent en suspens », p. 61 .

141. Les outils financiers développés dans le cadre des marchés de crédits d'émission en lien avec les politiques publiques de lutte contre le changement climatique n'ont pu en revanche être traités ici ; nous renvoyons sur cette question aux ouvrages généraux, notamment, Rotouillé J.-C., L'utilisation de la technique de marché en droit de l'environnement: l'exemple du système européen d'échange des quotas d'émission de gaz à effet de serre, LGDJ, BDP, 2017, Les instruments économiques au service du climat, centre d'analyse stratégique, Rapports et documents $n^{\circ} 45$, La documentation française, 2012, Mobiliser les financement pour le climat; une feuille de route pour une économie décarbonée, Grandjean A. et Canfin P., La documentation française, 2015.

142. Cf. infra Alidor B. "Compensation et services écosystémiques », Krajeski D. « Travail du sol, services écosystémiques et bail rural », Hoepffner H. « La commande publique de compensation environnementale : un impensé de la loi Biodiversité », Icher L. « La dépense publique en matière environnementale : l'exemple de la protection des sols et de ses services ».

143. $C f$. infra Jean S. «L'incidence des services écosystémiques en droit de la responsabilité civile». 



\title{
Le sol agricole, une ressource indispensable négligée
}

\author{
Ariane CHABERT \\ Docteure en agronomie \\ post-doctorante Idex T2SEC \\ Jean-Pierre SARTHOU \\ Professeur, INRA/INP-ENSAT
}

Si le sol et la nécessité de sa protection sont encore peu pris en compte par le droit, c'est peut-être parce qu'en premier lieu il a été négligé en agronomie ${ }^{1}$.

Dans un pays agricole comme la France, on se serait attendu à ce qu'au moins d'un point de vue agronomique, le sol soit reconnu comme une ressource centrale, tant elle est liée à nos productions, nos terroirs, notre patrimoine agricole.

Le monde agricole englobe une part dominante des acteurs du sol et si l'on considère ce dernier dans un espace continu allant des espaces très artificialisés que sont les sols urbains aux sols des espaces naturels protégés, le sol agricole a une place intermédiaire qu'il n'est pas possible d'isoler complètement ni des uns, ni des autres. De plus, du fait du lien étroit entre la « terre » et la production alimentaire, le sol agricole est certainement l'un des meilleurs exemples de l'importance de cette ressource pour l'humanité.

Le fait est que notre agriculture moderne est le résultat d'évolutions notamment scientifiques et industrielles qui ont participé à l'affaiblissement du lien entre le producteur et la terre qu'il cultive (I).

1. Sur la mobilisation de la science par le droit, cf. Naim-Gesbert E., Les dimensions scientifiques du droit de l'environnement. Contribution à l'étude des rapports de la science et du droit, Bruxelles, Bruylant, 1999. 
Parallèlement, les dommages faits au sol ont longtemps été ignorés et leur ampleur et conséquences restent encore méconnues (II). Finalement, la prise de conscience parmi les scientifiques, les praticiens, dans les sphères décisionnelles et associatives, que le sol est une ressource fragile et bien plus indispensable qu' on ne le pensait jusqu'au siècle dernier, est récente (III). Nos connaissances scientifiques sur les sols sont par ailleurs encore lacunaires et l'on ne dessine aujourd'hui encore que les contours des enjeux tant nationaux et qu'internationaux relatifs à la préservation du sol.

\section{L'ÉVOLUTION DE LA PERCEPTION DU SOL AGRICOLE}

Le lien étroit entre l'homme et le sol remonte aussi loin que les prémices de l'agriculture. L'évolution des sciences et de l'industrie, notamment d'après-guerre a cependant fondamentalement remanié notre rapport au sol (A). Néanmoins, les prises de conscience des dommages environnementaux liés à l'activité humaine ont fait naitre à la fin du siècle dernier la notion de sol-habitat (B), qui est aujourd'hui à la base d'un changement de paradigme dans la prise en compte de la santé des sols en agriculture (C).

A. DE L'ANTIQUITE AU XX ${ }^{\text {EME }}$ SIECLE : LA FORTE INFLEXION PROVOQUEE PAR LA SCIENCE ET L'INDUSTRIALISATION

Dès l'Antiquité, les agronomes romains posent les contours d'une compréhension de l'abondance et de la qualité des récoltes en relation avec le paysage et le sol, et les critères d'évaluation de ce dernier sont alors principalement la texture (composition et proportion des éléments constitutifs : argile, limons, sables) mais aussi la couleur, l'odeur et le goût (Camizuli et al., 2016). Les bases de ce que nous appelons aujourd'hui la fertilité d'un sol, de même que les règles empiriques permettant son amélioration et son entretien, sont dès lors jetées. Plus pragmatiquement, l'approche de la « qualité d'un champ » s'apprécie par le rapport entre les efforts nécessaires à sa mise en valeur et la production qui en est obtenue. Ces jugements permettent ainsi de définir une valeur économique des parcelles agricoles en vue d'échanges. Pendant des siècles, la notion de fertilité n'existait donc pas en tant que telle et l'on parlait simplement de ce qu'il fallait faire pour « bien cultiver ses terres ». Cette appréhension globale de la notion de fertilité des terres donna lieu aux révolutions agronomiques (ayant déjà permis de fortes 
augmentations de productivité surfacique et du travail) du MoyenÂge puis de la Renaissance, basées autant sur des inventions en machinisme que sur une meilleure utilisation de la biodiversité cultivée.

C'est au XIX ${ }^{\text {ème }}$ siècle, avec les travaux du chimiste allemand Justus von Liebig (1803-1873) qui révolutionne l'agriculture en identifiant les fondements de l'alimentation minérale des plantes, que la notion de fertilité du sol prend réellement corps et sera pendant des décennies associée à des caractéristiques chimiques des sols. Liebig oppose en effet aux théories dominantes de l'époque, essentiellement basées sur l'importance de l'humus, son éclairage nouveau sur l'alimentation végétale, l'identification des éléments essentiels à la croissance des plantes (l'azote, le phosphore et le potassium) et la notion de facteur minéral limitant ${ }^{2}$. Même si les découvertes de Liebig ont par la suite été critiquées, remises en cause et amendées, y compris par lui-même, elles sont à la base, il y a près de 180 ans, de l'industrialisation de l'agriculture. Liebig défendra notamment l'idée que le sol n'est pas la source de tous les éléments nécessaires à la plante (Brock, 1997). Les développements que connut l'agriculture suite à cette découverte ont conduit à une augmentation du rendement des cultures et les agriculteurs contraints de travailler de " mauvais sols » ont alors trouvé le moyen, chimique, de s'affranchir de cette contrainte. Mais ce bouleversement technique de la production agricole eut comme conséquence une augmentation de la dépendance de l'agriculture aux intrants industriels chimiques (Pfeiffer, 2006). Il eut comme autre conséquence, bien plus insidieuse, d'accélérer l'érosion des terres arables et de faire évoluer la perception du sol qui ne fut alors plus considéré comme une ressource indispensable au bon développement des plantes, puisque l'on pouvait, par l'ajout d'intrants, compenser là où le sol était défaillant.

Le sol est alors perçu par les nouvelles générations d'agriculteurs et particulièrement par ceux qui feront la révolution industrielle de l'après Seconde Guerre Mondiale, comme un « support » à la croissance des plantes, présentant des propriétés intrinsèques en termes de structure et de capacité à retenir l'eau et les minéraux qui doivent leur être apportés. On parle alors essentiellement de

2. Un élément minéral déficitaire limite la croissance de la plante. 
fertilité du sol. Le sol n'est reconnu que pour sa bonne ou mauvaise propension à porter les cultures. Cette fertilité est mesurée par une batterie d'indicateurs, toujours utilisés aujourd'hui, comprenant la richesse et la disponibilité en éléments nutritifs, la profondeur, la texture, la pierrosité... Elle représente l'ensemble des propriétés physico-chimiques d'un sol et donc l'ensemble des contraintes et avantages qu'il présente pour l'agriculteur. Un « bon » sol, ou sol fertile, est alors un sol sur lequel il est facile de produire et s'oppose au " mauvais » sol, qui ne sera productif que si l'on y apporte suffisamment de fertilisants, de travail du sol et d'eau. Ainsi, pendant la seconde moitié du $\mathrm{XX}^{\text {ème }}$ siècle et le début du $\mathrm{XXI}^{\mathrm{ème}}$, le sol a été perçu comme un support dont on pouvait améliorer les propriétés physiques et chimiques pour un meilleur développement des cultures, par le biais d'interventions culturales souvent lourdes et énergivores (labour et autres travaux du sol, drainage, fertilisation, amendements organiques et calciques...).

Dans les années 1980, la population mondiale grandissante, la limitation des ressources, l'instabilité sociale et les dégradations environnementales préoccupantes pour les processus naturels vitaux (Costanza et al., 1992), ont considérablement changé notre perception de l'agriculture et de la gestion des ressources naturelles. La santé des écosystèmes est alors devenue une préoccupation globale (Mermut and Eswaran, 1997) et le sol agricole ne fait pas exception. D'autant qu'on se rend alors compte que des sols jusqu'alors réputés «bons » perdent en fertilité. La puissance de traction nécessaire pour tirer la charrue augmente de 30 à $80 \mathrm{ch}$ traduisant une déstructuration et un tassement des sols devenus plus difficiles à travailler. En parallèle, l'apport d'azote nécessaire aux cultures pour atteindre de mêmes niveaux de rendement augmente continuellement, passant, au début des années 1960, de 60 à 75 unités de blé produites par unité d'azote de synthèse apportée, à seulement 20 à 30 au début des années 2000 (Tilman et al., 2002). Ces constats mettent en avant les limites de l'industrialisation de l'agriculture et ont incité les acteurs du monde agricole à reconcevoir leur vision du sol.

\section{B. LE XXI ${ }^{\text {ĖME }}$ SIÈCLE : L'ÉMERGENCE DE LA NOTION DE SOL-HABITAT}

Les différentiels de productivité observés malgré la fertilisation minérale croissante, sont pour l'essentiel imputable à une baisse de la capacité du sol à fournir une partie de l'alimentation azotée 
des plantes via le processus de minéralisation de l'humus ${ }^{3}$. Cette baisse est la conséquence de la diminution de la teneur des sols en humus, causée par des processus de destruction de ce dernier (i.e. par minéralisation) plus importants que les processus de son élaboration (i.e. par humification), essentiellement du fait d'un manque d'entretien organique des sols mais aussi d'un travail du sol trop important accélérant la minéralisation de l'humus.

Rapidement, les pratiques intensives, reposant essentiellement sur des intrants industriels, sont remises en cause. On commence alors à s'interroger sur l'importance des organismes du sol sur la structure et la fertilité de celui-ci (Dick, 1992) et l'impact des pratiques sur ces organismes. Néanmoins, trop peu de connaissances scientifiques permettent alors d'étayer la nature de la relation entre pratiques agricoles et faune du sol (Lee and Pankhurst, 1992). On ne parle désormais plus seulement de fertilité, mais de qualité des sols, définie en tant que «capacité d'un sol à fonctionner au sein des limites d'un écosystème pour supporter la productivité biologique, maintenir la qualité environnementale et promouvoir la santé des plantes et des animaux » (Doran et al., 1996). On réalise que le sol a sa propre vie et que les phénomènes de perte de fertilité s'expliquent par l'altération de l'activité des organismes du sol et des processus biologiques auxquels ils participent. Le sol est désormais considéré au travers de ses deux composantes : abiotique et biotique, soit inerte et vivante ; le sol est pour la première fois perçu comme un habitat. La qualité du sol ne dépend donc pas seulement des propriétés physiques et chimiques mais également de sa composante biologique et des fonctions qu'elle assure puisque de celles-ci découlent en grande partie les caractéristiques physicochimiques du sol dont dépend ensuite la croissance des plantes (Bender et al., 2016).

\section{La santé du SOL, un nOUVEaU PARADigme}

En même temps que la notion de qualité, est apparue la notion de santé du sol. Elle correspond entre autres à la stabilité de l'écosystème sol au travers de sa résilience au stress, sa diversité biologique et le niveau de recyclage interne des nutriments (Elliott and Lynch, 1994).

3. La minéralisation de l'humus est un processus physique, chimique et biologique menant à la transformation des constituants organiques en constituants minéraux (Gobat et al., 2013). 
La définition de la santé du sol ne fait cependant pas consensus au sein de la communauté scientifique et certains considèrent la santé du sol comme une composante de sa qualité, faisant uniquement référence à l'écologie du sol (Karlen et al., 1997; van Bruggen and Semenov, 2000). Plus généralement, santé et qualité sont considérées comme synonymes (Doran, 2002), même si la notion de santé est souvent préférée à celle de qualité car elle entretient une vision plus " vivante » du sol, plus dynamique, impliquant une approche holistique de ce dernier.

Le terme « santé » n'est pourtant pas anodin. Reconnaitre que le sol dispose d'une santé propre, c'est reconnaitre que son état peut être altéré. Les constats de perte de fertilité des sols qui ont conduit à l'émergence de ces termes ont en effet prouvé que les sols pouvaient perdre en fertilité sous la pression de l'activité humaine (Mason, 2003). De plus, la récente démocratisation de l'agriculture de conservation, considérant le sol comme une ressource fondamentale, a également montré que l'activité humaine est aussi en mesure de préserver et reconstituer la fertilité d'un sol, de le « soigner». Un certain nombre de pratiques sont aujourd'hui reconnues pour leur contribution à la bonne santé du sol (healthy soil management). Parmi elles, le non-travail du sol, la diversification des cultures de vente et intermédiaires (avec introduction de légumineuses), l'utilisation de couverts végétaux pendant l'interculture ainsi que l'utilisation d'amendements organiques sont reconnus pour contribuer notamment à une communauté microbienne des sols, active, diverse et source de services (Larkin, 2015). Cependant, les interactions entre pratiques et santé du sol sont encore très peu connues, et il reste beaucoup à faire au niveau scientifique. Il est notamment à ce jour impossible de proposer scientifiquement des réponses claires quant au type de pratiques permettant de maximiser les avantages sociologiques, économiques et écologiques liés au sol ainsi que sa productivité.

Pourtant sur le terrain, ces pratiques, apparues dans les années 70, se démocratisent rapidement depuis une vingtaine d'années et si l'utilisation des couverts peine encore à se développer, la réduction du travail du sol et l'allongement des rotations sont en revanche de plus en plus utilisés dans de nombreux pays du monde pour améliorer la fertilité naturelle des sols agricoles (Florentín et al., 2010; Larkin, 2015). 


\section{L'ABSENCE DE PRISE EN COMPTE DE L'IMPORTANCE DES PERTES DE SOL}

A cette lente prise en compte du sol comme habitat hébergeant une vie indispensable à la production agricole et comme ressource à préserver, s'ajoute une sous-estimation globale des risques encourus par les sols.

A l'instar du climat, le sol s'inscrit dans une double temporalité : avec 0,017 à $0,036 \mathrm{~mm}$ de sol formé par an (Reicosky, 2015), des millénaires sont nécessaires à la formation de la couche dite arable de $30 \mathrm{~cm}$ d'épaisseur (de 8300 à 17600 ans !) alors qu'il peut subir des dégradations irrémédiables à l'échelle humaine, en quelques décennies voire quelques années. Parmi les menaces pesant sur le sol, l'érosion, hydrique notamment, est l'une des plus sévères, avec l'artificialisation et l'imperméabilisation qui sont considérées par le Partenariat mondial des sols comme les principales menaces en Europe. Les autres menaces sont la contamination, le tassement, la perte de matières organiques, la perte de biodiversité, les glissements de terrain et l'acidification (Chenu et al., 2016; FAO and ITPS, 2015; Jones et al., 2012).

Bien que l'on estime qu'à l'heure actuelle en France le sol se détruit 40 fois plus vite qu'il ne se crée, l'importance de l'érosion a longtemps été sous-estimée par les agriculteurs et les acteurs du monde agricole en général car ce phénomène est pernicieux. Même si la perte effective de sol peut se constater au travers de hauts de coteaux de plus en plus blancs à mesure que la roche mère se rapproche de la surface, l'érosion ne s'observe finalement que ponctuellement lors de forts épisodes orageux (fossés comblés de terre et cours d'eau chargés de limons et d'argile). Une fois la culture en place, les dégradations sur le sol ne sont en effet presque plus directement visibles. Dans les grands bassins céréaliers européens, au relief relativement plat, le sol est profond et l'érosion plus lente (mais réelle) qu'en zones de culture sur coteaux. Le sol n'y disparait pas « à vue d'œil » et les régulières opérations de curage des fossés permettent en général de récupérer la terre perdue. Ainsi, jusqu'à récemment, le phénomène érosif passait presque inaperçu, tout au moins ne marquait-il pas les esprits.

C'est la prise de conscience que ces pertes de sols sont à l'origine d'une perte de fertilité et donc de rentabilité, qui a poussé les acteurs 
du monde agricole à s'inquiéter de l'érosion notamment, et des altérations physiques et chimiques faites au sol plus généralement. On associe à l'érosion seule des pertes de rendement de l'ordre de 0,3 \% (den Biggelaar et al., 2003; Scherr, 2003). A cela s'ajoute le transfert de fertilisants, qui en plus de représenter une perte nette pour les agriculteurs, altère la qualité de l'eau et met en danger les écosystèmes aquatiques. Les pertes de rendement et les surcoûts de production représentés par les autres altérations physiques et chimiques des sols agricoles ne sont à notre connaissance pas chiffrés mais il est permis de penser qu'ils représentent un impact économique important.

\section{LES LIMITES DES CONNAISSANCES SCIENTIFIQUES ACTUELLES}

Notre connaissance du sol est encore lacunaire. Bien que sous nos pieds, cet écosystème est difficile à étudier et observer, nous n'en connaissons encore que très peu de choses (A) d'autant que la recherche scientifique sur les sols, bien qu'en pleine expansion est récente $(B)$ et manque encore d'outils, notamment d'indicateurs de leur santé $(\mathrm{C})$.

\section{A. LE SOL : TROISIÈME FRONTIÈRE BIOTIQUE.}

Le sol est considéré comme étant la $3^{\text {ème }}$ frontière biotique, c'est-àdire l'un des milieux dont nous n'avons pas encore exploré toute la richesse, après les fonds océaniques et la canopée tropicale, du fait de sa complexité, de son inaccessibilité et de son impressionnante diversité. On considère que le sol renferme $25 \%$ des espèces décrites (Decaëns et al., 2006) et probablement une part plus importante encore des espèces non décrites. Pour comparaison, dans un pâturage supportant en moyenne sur une année 3 unités de gros bétail ${ }^{4}$ par hectare, la biomasse des seuls vers de terre est presque comparable à celle des bovins ${ }^{5}$ (Gobat et al., 2013). Or les vers de terre sont l'un des groupes les moins abondants de la faune du sol, se comptant en dizaines ou centaines par mètre carré lorsque les nématodes, acariens et collemboles se comptent en milliers ou en millions, sans

4. L'UGB "vaut" par définition une vache laitière standard de $600 \mathrm{~kg}$, et un mouton représente par exemple 0,15 UGB.

5.1000 à $1500 \mathrm{~kg} / \mathrm{ha}$ de vers de terre pour $1800 \mathrm{~kg} / \mathrm{ha}$ de bovins. 
parler des champignons et bactéries qui se comptent en milliards au mètre carré... Pourtant la biodiversité du sol reste globalement méconnue, autant sur le plan taxonomique qu'écologique (structure des réseaux trophiques notamment), car au-delà de l'extraordinaire diversité des formes de vie qu'il renferme, l'approche structurelle et fonctionnelle de la biodiversité en général et de celle du sol en particulier est très récente. Cela traduit le faible intérêt scientifique porté ces dernières décennies à la biodiversité du sol, notamment agricole, probablement du fait de notre ignorance des avantages fonctionnels (pour l'alimentation hydrominérale et la protection contre les bioagresseurs des plantes notamment) qu'elle peut apporter aux cultures.

\section{B. InVENTAIRE ET MULTIFONCTIONNALITÉ DES SOLS : UNE COMMUNAUTÉ SCIENTIFIQUE RÉCENTE}

La Charte européenne sur les sols de 1972 proposait déjà la prise en compte de la multifonctionnalité des sols, mais en pratique la fertilité des sols restait encore la principale préoccupation des agronomes. En France, le programme Gessol lancé en 1998, est à l'initiative de la structuration d'une communauté scientifique autour de la multifonctionnalité des sols et entreprend, dans les années 2000, de considérer et recenser les dégradations physiques, chimiques et biologiques faites au sol et leurs impacts sur les fonctions assurées par celui-ci (Bispo et al., 2016; Citeau et al., 2008). Clôturé en 2015, ce programme a grandement participé au développement des connaissances liées aux sols, en particulier les changements d'usage et leur gestion en réponse aux questions de sécurité alimentaire, changement climatique, santé et biodiversité. Ces données doivent désormais faire l'objet de synthèses, confrontations et diffusions pour améliorer la gestion des sols et leur permettre une meilleure protection. De plus, malgré cette importante acquisition de connaissances au cours des 20 dernières années, des lacunes persistent en termes de connaissances scientifiques sur les sols, notamment en agronomie. Parmi les enjeux de recherche dans les années à venir, le programme Gessol identifie le rôle des sols dans le maintien voire l'augmentation de la production agricole, dans l'atténuation du changement climatique et l'adaptation des systèmes de production à ce dernier, et les bonnes pratiques de gestion des sols et leur mise en œuvre (Bispo et al., 2016). 
Parmi les initiatives françaises, on notera également le Groupement d'intérêt scientifique Sol $\left(\mathrm{Gis} \mathrm{Sol}^{6}\right)$, qui depuis 2001 conçoit, oriente et coordonne l'inventaire géographique des sols de France, le suivi de leurs propriétés et l'évolution de leurs qualités. Au niveau international, la première évaluation globale de la ressource sol et des menaces pesant sur elle date de 2015 (FAO and ITPS, 2015). Par ailleurs, l'estimation de l'étendue des dégradations est toujours considérée comme une opération délicate à l'échelle globale (Gibbs and Salmon, 2015).

Enfin, avec l'apparition du concept de « One world, one health », proposé par la Wildlife Conservation Society en 2004 (WCS, 2004) et repris par de grandes organisations internationales comme la FAO et l'UNICEF en 2008 (FAO et al., 2008), la question du lien entre santé humaine, santé des animaux et santé de l'environnement est devenu un axe de recherche pluridisciplinaire émergent. Pourtant la santé du sol a peiné à trouver sa place dans cette approche holistique de la santé, où elle n'est que très indirectement prise en compte notamment via les types d'occupation et pour son rôle de réservoir de pathogènes (Rabinowitz and Conti, 2013). Ce n'est que très récemment que son importance a été relevée par la communauté scientifique et certains auteurs proposent même de reconnaitre le sol comme le principal pivot entre les concepts de services écosystémiques et de «one health » (Keith et al., 2016), la très grande majorité des approches par services écosystémiques n'intégrant pas encore systématiquement de lien vers la santé humaine (Ford et al., 2015). Cependant, si les relations entre pratiques agricoles, santé du sol et santé des plantes commencent à être petit à petit élucidées, celles entre santé du sol, qualités nutritionnelles des produits alimentaires et santé humaine restent très peu comprises et étudiées (Reeve et al., 2016).

\section{A LA RECHERCHE D'INDiCATEURS PERTINENTS}

La vision holistique du sol qui se répand depuis ces quinze dernières années a nécessité le développement de nouveaux indicateurs pour apprécier non plus seulement la fertilité du sol, mais sa qualité voire sa santé. La recherche scientifique s'est alors fortement orientée vers la recherche d'indicateurs notamment biologiques (de Paul Obade and Lal, 2016; Doran and Zeiss, 2000; Pankhurst et al., 1995) 
permettant le pilotage de systèmes agricoles préservant le sol en tant que ressource, tout en maximisant le profit des agriculteurs par l'optimisation de l'utilisation des intrants industriels (carburants, fertilisants, eau d'irrigation, pesticides, machinisme spécifique).

Des mallettes d'indicateurs, d'outils et de protocoles ont vu le jour en réponse à la demande croissante des acteurs soucieux de prendre en compte la santé du sol, notamment dans l'évaluation de l'effet des pratiques agricoles (Stockdale and Watson, 2009). Cependant, la notion de santé du sol étant intrinsèquement difficile à préciser du fait qu'elle recouvre l'intégralité des propriétés et fonctions du sol, son évaluation reste toujours délicate, sujette à débat et variable selon le contexte et le type de sol. La recherche explore encore chaque année de nouvelles pistes pour l'évaluation de la santé du sol, au travers des avancées technologiques en matière de biologie moléculaire (Arias et al., 2005), via la recherche de nouveaux indicateurs physicochimiques en relation avec la matière organique (Morrow et al., 2016) ou encore par l'exploration d'indicateurs jusqu'alors ignorés pour le sol comme le potentiel d'oxydo-réduction (Husson, 2013; Husson et al., 2016). 


\section{Bibliographie}

Arias, M.E., Gonzalez-Pérez, J.A., Gonzalez-Vila, F.J., Ball, A.S., 2005. Soil health - a new challenge for microbiologists and chemists. International Microbiology, 8, 13-21.

Bender, S.F., Cameron Wagg, C., van der Heijden, M.G.A., 2016. An Underground Revolution: Biodiversity and Soil Ecological Engineering for Agricultural Sustainability. Trends in Ecology and Evolution, 31, 440-452.

Bispo, A., Guellier, C., Martin, E., Sapijanskas, J., Soubelet, H., Chenu, C., 2016. Les sols. Intégrer leur multifonctionnalité pour une gestion durable, Collection Savoir-faire, éditions Quae, Versailles, $384 \mathrm{p}$.

Brock, W.H., 1997. Justus von Liebig : the chemical gatekeeper, Cambridge University Press, Cambridge, 396 p.

Camizuli, E., Petit, C., Bernigaud, N., Reddé, M., 2016. Principes méthodologiques pour caractériser des agrosystèmes antiques. Les Nouvelles de l'archéologie 142, 20-26.

Chenu, C., Bispo, A., Martin, E., Sapijanskas, J., Soubelet, H., 2016. Sols et société : enjeux actuels. In : Les Sols. Intégrer Leur Multifonctionnalité Pour Une Gestion Durable (A. Bispo, C. Guellier, E. Martin, J. Sapijanskas, H. Soubelet, C. Chenu, coord.), Collection Savoir-faire, éditions Quae, Versailles, $15-53$.

Citeau, L., Bispo, A., Bardy, M., King, D., coord., 2008. Gestion durable des sols, Collection Savoir-faire, éditions Quae, Versailles, $320 \mathrm{p}$.

Costanza, R., Norton, B.G., Haskell, B.D., 1992. Ecosystem Health: New Goals for Environmental Management. Island Press, Washington, DC, $269 \mathrm{p}$.

de Paul Obade, V., Lal, R., 2016. Towards a standard technique for soil quality assessment. Geoderma 265, 96-102.

Decaëns, T., Jimenez, J.J., Gioia, C., Measey, J., Lavelle, P., 2006. The values of soil animals for conservation biology. European Journal of Soil Biology, 42, S23-S38.

den Biggelaar, C., Lal, R., Eswaran, H., Breneman, V., Reich, P., 2003. Crop yield losses to soil erosion at regional and global scales: Evidence from plot-level and GIS data. In : Land Quality, Agricultural Productivity, and Food Security: Biophysical Processes and Economic Choices Al Local, Regional, and Global Levels (K. Wiebe, ed.), Edward Elgar Publishing, Cheltenham, UK, 262-279. 
Dick, R.P., 1992. A review: long-term effects of agricultural systems on soil biochemical and microbial parameters. Agriculture, Ecosystems and Environment, 40, 25-36.

Doran, J.W., 2002. Soil health and global sustainability: translating science into practice. Agriculture, Ecosystems and Environment, $88,119-127$.

Doran, J.W., Sarrantonio, M., Liebig, M., 1996. Soil health and sustainability. In : Advances in Agronomy (D.L. Sparks, ed.), Academic Press, San Diego, 1-54.

Doran, J.W., Zeiss, M.R., 2000. Soil health and sustainability: managing the biotic component of soil quality. Applied Soil Ecology, 15, 3-11.

Elliott, L.F., Lynch, J.M., 1994. Biodiversity and soil resilience. In : Soil Resilience and Sustainable Land Use (D.J. Greenland, I. Szabolcs, eds.), CAB International, Wallingford, UK, 353-364.

FAO, OIE, WHO, UN System Influenza Coordination, UNICEF, BANK, W., 2008. Contributing to One World, One Health. A Strategic Framework for Reducing Risks of Infectious Diseases at the Animal-Human-Ecosystems Interface, Consultation Document, $67 \mathrm{p}$.

Florentín, M.A., Peñalva, M., Calegari, A., Derpsch, R., 2010. Green manure / cover crops and crop rotation in Conservation Agriculture on small farms. Integrated Crop Management, 12, $1-109$.

FAO, ITPS, 2015. Status of the World's Soil Resources - Main Report, Food and Agriculture Organization of the United Nations and Intergovernmental Technical Panel on Soils, Rome, 650 p.

Ford, A.E.S., Graham, H., White, P.C.L., 2015. Integrating Human and Ecosystem Health Through Ecosystem Services Frameworks. Ecohealth, 12, 660-671.

Gibbs, H.K., Salmon, J.M., 2015. Mapping the world's degraded lands. Applied Geography, 57, 12-21.

Gobat, J.-M., Aragno, M., Matthey, W., 2013. Le sol vivant. Bases de pédologie - Biologie des sols, $3^{\text {ème }}$ édition, Collection : Science et ingénierie de l'environnement, Presses Polytechniques Romandes, $820 \mathrm{p}$.

Husson, O., 2013. Redox potential (Eh) and pH as drivers of soil/ plant/microorganism systems: a transdisciplinary overview pointing to integrative opportunities for agronomy. Plant and Soil, 362, 389-417.

Husson, O., Husson, B., Brunet, A., Babre, D., Alary, K., Sarthou, J.P., Charpentier, H., Durand, M., Benada, J., Henry, M., 2016. 
Practical improvements in soil redox potential (Eh) measurement for characterisation of soil properties. Application for comparison of conventional and conservation agriculture cropping systems. Analytica Chimica Acta 906, 98-109.

Jones, A., Panagos, P., Barcelo, S., Bouraoui, F., Bosco, C., Dewitte, O., Gardi, C., Erhard, M., Hervas, J., Hierderer, R., Jeffery, S., Lükewille, A., Marmo, L., Montanarella, L., Olazabal, C., Petersen, J.-E., Penizek, V., Strassburger, T., Toth, G., Van den Eeckhaut, M., Van Liedekerke, M., Verheijen, F., Viestova, E., Yigini, Y., 2012. The state of soil in Europe: A contribution of the JRC to the European Environment Agency's environment state and outlook report, SOER 2010, Reference report, Luxembourg, $72 \mathrm{p}$.

Karlen, D.L., Mausbach, M.J., Doran, J.W., Cline, R.G., Harris, R.F., Schuman, G.E., 1997. Soil quality: a concept, definition, and framework for evaluation (a guest editorial). Soil Science Society of America Journal, 61, 4-10.

Keith, A.M., Schmidt, O., McMahon, B.J., 2016. Soil stewardship as a nexus between Ecosystem Services and One Health. Ecosystem Services, 17, 40-42.

Larkin, R.P., 2015. Soil Health Paradigms and Implications for Disease Management. Annual Review of Phytopathology, 53, 199-221.

Lee, K.E., Pankhurst, C.E., 1992. Soil organisms and sustainable productivity. Australian Journal of Soil Research, 30, 855-892.

Mason, J., 2003. Sustainable Agriculture, $2^{\text {nd }}$ edition, Landlinks Press, $205 \mathrm{p}$.

Mermut, A.R., Eswaran, H., 1997. Opportunities for soil science in a milieu of reduced funds. Canadian Journal of Soil Science, 77, $1-7$.

Morrow, J.G., Huggins, D.R., Carpenter-Boggs, L.A., Reganold, J.P., 2016. Evaluating Measures to Assess Soil Health in LongTerm Agroecosystem Trials. Soil Science Society of America Journal, 80, 450-462.

Pankhurst, C.E., Hawke, B.G., McDonald, H.J., Kirkby, C.A., Buckerfield, J.C., Michelsen, P., O’Brien, K.A., Gupta, V.V.S.R., Doube, B.M., 1995. Evaluation of soil biological properties as potential bioindicators of soil health. Australian Journal of Experimental Agriculture, 35, 1015-1028.

Pfeiffer, D.A., 2006. Eating fossil fuels: oil, food and the coming crisis in agriculture. New Society Publishers, Gabiola Island, Canada, $144 \mathrm{p}$. 
Rabinowitz, P., Conti, L., 2013. Links Among Human Health, Animal Health, and Ecosystem Health. Annual Review of Public Health, 34, 189-204.

Reeve, J.R., Hoagland, L.A., Villalba, J.J., Carr, P.M., Atucha, A., Cambardella, C., Davis, D.R., Delate, K., 2016. Organic Farming, Soil Health, and Food Quality: Considering Possible Links. Advances in Agronomy, 137, 319-368.

Reicosky, D.C., 2015. Conservation tillage is not conservation agriculture. Journal of Soil and Water Conservation, 70, 103A-108A.

Scherr, S.J., 2003. Productivity-related economic impacts of soil degradation in developing countries: An evaluation of regional experience. In: Land Quality, Agricultural Productivity, and Food Security: Biophysical Processes and Economic Choices Al Local, Regional, and Global Levels (K. Wiebe, Ed.), Edward Elgar Publishing, Cheltenham, UK, 231-261.

WCS, 2004. One World, One Health: Building Interdisciplinary Bridges to Health in a Globalized World. Symposium organized by the Wildlife Conservation Society, 29th September at The Rockefeller University, New York City.

Stockdale, E.A., Watson, C.A., 2009. Biological indicators of soil quality in organic farming systems. Renewable Agriculture and Food Systems, 24, 308-318.

Tilman, D., Cassman, K.G., Matson, P.A., Naylor, R., Polasky, S., 2002. Agricultural sustainability and intensive production practices. Nature, 418, 671-677.

Van Bruggen, A.H.C., Semenov, A.M., 2000. In search of biological indicators for soil health and disease suppression. Applied Soil Ecology, 15, 13-24. 



\title{
Propriété et protection des sols Réflexions civilistes sur la prise en compte de la qualité des sols
}

\author{
Lionel BOSC
}

Doctorant, IEJUC

«L'histoire du droit de propriété est un des plus grands objets d'étude qui puissent être offerts à la philosophie et à l'érudition [...] une savante analyse reste à faire, qui présente dans un vaste ensemble, l'action de l'humanité sur les biens de la terre et l'influence de l'appropriation, sur la formation et le développement progressif des sociétés $\rangle^{1}$. Propriété et terre connaissent une relation profonde, ancienne, et néanmoins mal connue ; a contrario de l'eau et l'air dont les études semblent plus nombreuses ${ }^{2}$.

Or, juridiquement, une différence fondamentale oppose l'eau et l'air à la terre, donc au sol. En effet, l'eau et l'air sont considérés comme des choses communes ${ }^{3}$ et sont à ce titre inappropriables, notamment par l'absence d'utilité à en faire des objets de propriété. N'étant

\footnotetext{
1. Giraud C.-J.-B., Recherches sur le droit de propriété chez les romains, sous la République et sous l'Empire, éd. Aubin, 1838, p. 13.

2. V. par ex. Joachim C., Le partage des compétences en matière de protection de la qualité des eaux douces au Canada et dans l'Union européenne, Th. Toulouse 1, 2014 ; Barbara J., Les normes relatives à la réduction de la pollution de l'air et la sanction des faits de pollution, Th. Grenoble II, 1997 ; Directive n ${ }^{\circ}$ 2008/50/CE du 21/05/08 concernant la qualité de l'air ambiant et un air pur pour l'Europe ; Directive 2000/60/CE du Parlement européen et du Conseil du 23 octobre 2000 établissant un cadre pour une politique communautaire dans le domaine de l'eau.

3. V. art. 714 C. civ. : "Il est des choses qui n'appartiennent à personne et dont l'usage est commun à tous ». Sur la question, V. Chardeaux M.-A., Les choses communes, L.G.D.J., 2006.
} 
pas rares, en effet, il n'apparaît pas nécessaire de les réserver ${ }^{4}$. A contrario, le sol est certainement le véritable objet premier du droit de propriété. A ce titre, il est considéré, aux termes de l'article 518 du Code civil, comme un «immeuble par nature » : les « fonds de terre », qui « comprennent la surface du sol et le sous-sol $»^{5}$, « constituent [en effet] l'immeuble par excellence $»^{6}$. Pourtant, la définition juridique du sol parait faire défaut, notamment en droit civil. Si certaines propositions doctrinales existent ${ }^{7}$, notamment en raison de la nécessité de la protection de l'environnement, il convient de relever que le Code civil, alors qu'il est structuré par la propriété ${ }^{8}$, reste étonnamment silencieux sur son objet principal qu'est le sol. Le Code civil traite effectivement le sol moins en tant qu'objet de droit, qu'au travers du régime de la propriété elle-même.

La propriété s'est pourtant construite essentiellement autour des immeubles, ces derniers connaissant initialement la valeur la plus grande. C'est, par exemple, la propriété du sol qui permet, par l'effet de l'accession que l'on retrouve aujourd'hui à l'article $546 \mathrm{du}$ Code civil', de s'approprier les constructions réalisées sur l'assiette définie par le sol. Aussi est-il évident que la propriété ne se limite pas au sol. Bien au contraire, l'alinéa premier de l'article $552 \mathrm{du}$ Code civil énonce que "la propriété du sol emporte propriété du dessus et du dessous ", c'est-à-dire que la propriété du sol permet l'appropriation de tout ce qui se trouve dessus mais également de tout ce qui se trouve dessous. Il convient de noter ici qu'il existe quelques exceptions : le Code civil comporte des dérogations pour les trésors ${ }^{10}$,

4. « Le droit considère qu'il n'est pas légitime ou utile d'en réserver leur usage à une seule personne et qu'elles doivent être destinées à l'usage commun » : Rochfeld J., Les grandes notions de droit privé, PUF, Paris, 4 e éd., spéc. p. 215.

5. Terre F. et Simler Ph., Les biens, Précis, D., $9^{\mathrm{e}}$ éd. 2014, n 33, p. 40.

6. Idem.

7. V. par ex. Neyret L. et Martin G. J., (dir.), Nomenclature des préjudices environnementaux, L.G.D.J., 2012, spéc. p.16.

8. M. Zenati-Castaing relève en effet « l'omniprésence de la notion de propriété dans le Code [civil] n'est pas un résidu de phraséologie révolutionnaire ni le souvenir d'un prurit passager de propriétarisme. Elle est le produit de l'art législatif légendaire des membres de la commission du gouvernement du Consulat, dont on connait l'esprit modéré », ZenatiCastaing F., La propriété, mécanisme fondamental du droit, RTD Civ. 2006, p. 445.

9. Art. 546 c. civ. : «La propriété d'une chose soit mobilière, soit immobilière, donne droit sur tout ce qu'elle produit, et sur ce qui s'y unit accessoirement soit naturellement, soit artificiellement. Ce droit s'appelle "droit d'accession". »"

10. V. Art. 716 c. civ. qui prévoit le trésor comme un accessoire au propriétaire du fonds, mais envisage également une forme de récompense pour celui qui le découvre. V. sur le sujet, Berchon P., Trésor, Rép. civ. Dalloz, janv. 2009. 
ou encore s'agissant des ressources minières et autres carrières ${ }^{11}$, principalement pour des raisons de souveraineté ${ }^{12}$. Cependant, si l'article 552 permet de présumer la propriété du dessus et du dessous, quitte à ce que cette présomption soit renversée ou inversée, il n'en reste pas moins que le Code civil conditionne cette présomption à la préalable propriété du sol. À la fin, il apparaît que la propriété du sol n'est pas traitée directement par le Code. En ce sens, l'article 552 du Code civil ne fait que présupposer l'appropriation de son objet - principal - qu'est le sol, afin de déterminer l'appropriation du sous-sol (et du dessus) et, indirectement on le verra, sa ou ses qualité(s). Il n'en reste pas moins que l'appropriation du sol demeure le véritable enjeu de la propriété.

L'enjeu principal et décisif du droit, pendant des siècles, reposait déjà sur l'appropriation des « terres ». La propriété s'est, en effet, largement construite par le droit romain qui voyait « les biens en tant que tels $\rangle^{13}$, entendu que " l'élaboration du droit se [faisait] à partir d'une contemplation du réel, qui se compose essentiellement des personnes et des choses $\|{ }^{14}$; alors que nous avons tendance, de nos jours, à « voir les biens à travers le prisme des personnes sous l'influence de la philosophie individualiste des droits subjectifs $»^{15}$. Aussi la propriété a-t-elle constitué, " dès l'Ancien Régime, la forme emblématique de la maitrise du sol, pour les paysans comme pour les notables $\gg{ }^{16}$. Ainsi la propriété immobilière, qui connaissait initialement un lien indépassable avec sa connexion au sol, constituait-elle la "véritable " propriété. A contrario, la propriété mobilière, structurée essentiellement autour de la liberté du commerce, apparaissait peu importante comme en témoigne l'adage res mobilis, res vilis ${ }^{17}$. Or, si la propriété d'une chose se définit par « la qualité qu'a un bien d'appartenir à une personne $»^{18}$,

11. V. Code minier ; Sur la définition des mines et carrières, V. Art. L 100-1 du Code minier : "L'assujettissement d'un gîte contenant des substances minérales ou fossiles soit au régime légal des mines, soit à celui des carrières est déterminé par la seule nature des substances qu'il contient, sous réserve de dispositions contraires prévues par le présent Code».

12. V. Loi du 21 avril 1810 concernant les mines, les minières et les carrières.

13. Zenati-Castaing F. et Revet T., Les biens, PUF, Paris, 2008, 3éd., nº 2, p. 24.

14. Idem.

15. Idem.

16. Sicard G., Le droit de propriété avant l'article 17 de la déclaration des droits de l'homme et du citoyen, in Propriété et Révolution, éd. CNRS, Toulouse 1, 1990, p. 20.

17. "Chose mobilière, chose vile»

18. Zenati-Castaing F. et Revet T., Les biens, op. cit. $\mathrm{n}^{\circ}$ 2, p. 24. 
c'est-à-dire « le droit d'en tirer non pas certains services déterminés, [...] mais tous les services qui sont libres $»^{19}$, alors il apparaît que la qualité du sol n'est qu'une de ses utilités parmi d'autres ${ }^{20}$. Il s'en déduit que la ou les qualité(s) du sol, parce qu'elle(s) participe(nt) de la nature de la chose - objet de droit - qu'est le sol, ne peu(ven) t être distinguée(s) de la chose elle-même ${ }^{21}$. Dès lors, traiter de la propriété du sol, c'est déjà traiter de la propriété de ses qualités, et inversement.

Il semble que les liens qui unissent la propriété et le sol soient quasi-indivisibles, pour ne pas dire plus sans tirer de conclusions hâtives. En effet, la lecture du Code civil nous laisse à penser que le sol, et conséquemment sa qualité, est essentiellement considéré soit comme le support du travail - de la terre - dans une société agraire $^{22}$, soit comme le support de constructions notamment d'habitation. Dès lors, il n'est pas étonnant que la qualité du sol soit peu considérée, encore que certaines qualités aient pu déjà motiver l'absence d'appropriation de certains espaces : nous penserons aux zones régulièrement inondées, excluant de fait toutes constructions; encore aux zones excessivement pierreuses, souvent infertiles. Or, tout le paradoxe éclot ici : alors même que le sol apparaît être l'objet privilégié de la propriété, il ne semble être considéré que comme support de celle-ci, méconnaissant de fait ses qualités. Notre réflexion portera ainsi, sur ces bases, sur la protection de la qualité du sol par la propriété. Aussi devons-nous nous interroger, non seulement, sur la possibilité que la propriété protège la qualité des sols (II) mais aussi, préalablement, sur l'appréhension du sol par la propriété en tant qu'objet ignorant ses qualités (I), puisque tel est le socle de la prise en compte de la qualité par le droit de propriété.

19. De Vareilles-Sommieres, La définition et la notion juridique de la propriété, RTD civ., 1905, p. 443 s. : l'auteur utilise de manière indifférenciée ici les termes « service » et «utilité ».

20. V. Beaussonie G., La qualification juridique des services écosystémiques, supra.

21. Sur la notion, V. Beaussonie G., La qualification juridique des services écosystémiques, supra.

22. Le Code Civil a été promulgué en 1804, bien avant la Révolution Industrielle ; de plus, ce Code connait un fort héritage du droit romain, édicté dans une société bien plus agraire encore s'il en est. 


\section{LA QUALITÉ DU SOL NÉGLIGÉE PAR LA PROPRIÉTÉ}

Si les liens entre la terre et la propriété ont été brièvement rappelés, l'analyse de cette relation mérite d'être approfondie. En effet, il nous semble que le sol est certainement le support d'expression historique de la propriété (A), à tel point qu'il en est également, le support structurant la propriété (B).

\section{A. LE SOL, SUPPORT HISTORIQUE D’EXPRESSION DE LA PROPRIÉTÉ}

Les origines de la propriété en tant que phénomène social ont pu faire l'objet de vastes débats chez les anthropologues ${ }^{23}$, chez les juristes ${ }^{24}$, ou encore chez les philosophes ${ }^{25}$, pour ne citer qu'eux. Pourtant, les liens profonds entre l'apparition de la propriété et celle de l'agriculture en tant que culture primaire des sols, c'est-à-dire l'émergence de la domestication des végétaux - donc du travail de la terre -, font l'objet d'un consensus assez large, quand bien même les relations de cause(s) à effet(s) sont largement discutées. " Il fallait mettre de côté une partie de la récolte pour semer la saison suivante et s'assurer que personne ne mange les réserves. Les premiers semis ont dû être effectués autour des maisons. Mais ensuite [les hommes] ont cherché d'autres endroits privilégiés : clairières, alluvions de cours d'eau qui débordent chaque année... Il a donc fallu imposer

23. V. not. Le Roy E., La terre de l'autre. Une anthropologie des régimes d'appropriation foncière, L.G.D.J., Paris, 2011 ; Testart A., Propriété et non-propriété de la Terre, Études rurales, 165-166, 2003, 209-242 ; Testart A., Propriété et non-propriété de la terre, Études rurales, 169-170, 2004, 149-178 ; Godelier M., Aux sources de 1'anthropologie économique, Socio-anthropologie, 7, 2000, http://socio-anthropologie.revues.org/98; spéc. $\mathrm{n}^{\circ} 13$; Müller B., Anthropologie de la propriété et du pouvoir : des utopies alternatives au néolibéralisme 2, Annuaire de l'EHESS, 2010, 606-607.

24. V. not. Carbonnier J., Flexible droit, Pour une sociologie du droit sans rigueur, L.G.D.J., $10^{\mathrm{e}}$ éd., 2014 ; Xifaras M., La propriété, étude de philosophie du droit, PUF, 2004 ; Simler $\mathrm{Ph}$., Qu'est-ce que la propriété, in Qu'en est-il de la propriété ? L'appropriation en débat, Les Travaux de l'IFR, Mutation des normes juridiques n 5, PUT, 2006, p. 251 et s. ; Zénati F., La nature juridique de la propriété, contribution à la théorie du droit subjectif, th. Lyon III, 1981 ; Lévy J.-P., Histoire de la propriété, par, coll. « Que sais-je ?», PUF, 1972 .

25. V. not. Proudhon P.J., Qu'est-ce que la propriété ?, Les classiques de la Philosophie, LGF, 2009 ; Proudhon P.-J., Théorie de la propriété, Les introuvables, L'Harmattan, 1997 ; Engels F., L'origine de la famille, de la propriété privée et de l'Etat, Le temps des cerises, 2012, préf. Darmangeat C.; Rousseau J.J., Discours sur l'origine et le fondement des inégalités parmi les hommes, Flammarion, 2011 ; Tocqueville A., De la démocratie en Amérique, Gallimard, 1986 ; Dagognet F., Philosophie de la propriété, l'avoir, PUF, 1992 ; Mounier E., De la propriété capitaliste à la propriété humaine, Desclée de Brouwer, Paris, 1936. 
un droit de propriété sur la récolte au beau milieu de la nature. Là où, précédemment, tout le monde avait le droit de le cueillir. Ce qui a dû être le plus difficile à inventer, ce n'est pas l'agriculture, c'est la société qui va avec $\gg^{26}$. La propriété serait ainsi apparue par la domestication des végétaux par l'effet d'une certaine nécessité : le passage de sociétés humaines basées sur la chasse et la cueillette vers des sociétés où les aléas de subsistance se verraient diminués par la culture de végétaux, c'est-à-dire de manière pragmatique par la culture des sols. L'appropriation des terres serait donc la conséquence de son travail : "Le travail est un exercice de la liberté ; la propriété est la conséquence du travail. Ainsi la propriété est un droit inhérent à la liberté elle-même $»^{27}$.

Mieux saisir les relations entre le sol et la propriété, c'est comprendre le constat que nous avons préalablement établi : le Code civil, dominé dans son ensemble par la propriétée ${ }^{28}$, ne s'intéresse, de façon paradoxale, qu'indirectement au sol ; au mieux le traite-til, de manière indirecte donc, en tant qu'objet présupposé au sein de l'article 552 par exemple ou, plus généralement, tire-t-il les conséquences de son appropriation ${ }^{29}$. De la même manière, l'article 553 du Code civil ${ }^{30}$ vient présumer la propriété de ce qui se trouve « dessus » sans se référer au sol mais au « terrain » : ici, la propriété du sol est présupposée sans pour autant être expressément citée. Ce ne sont donc que les conséquences de son appropriation qui sont traitées. Pourtant, en se référant à la propriété du terrain, il s'agit bien de viser la propriété du sol. À titre d'exemple, la propriété d'une maison est juridiquement bien plus déterminée par la propriété du sol sur lequel elle construite que par l'appréhension de la maison elle-même. C'est parce que le sol est préalablement approprié que, conséquemment, la maison l'est aussi. " Prétendre que les droits de la propriété, les vrais fondements de la société civile, n'effleurent

26. Mazoyer M., Pelt J.-M, Monod T., Girardon J., La plus belle histoire des plantes, éd. Points, 2002.

27. Projet de déclaration soumis au bureau de l'Assemblée par le député Pison de Galland, in La déclaration des droits de l'homme et du citoyen, S. Rials, Paris, Hachette, 1988.

28. Zenati-Castaing F., La propriété, mécanisme fondamental du droit, RTD Civ. 2006, p. 445.

29. V. Art. 554, 624, 660 et $661,1245-2,1601-3,1792$ et 1793 C. civ.

30. Art. 553 du c. civ. : "Toutes constructions, plantations et ouvrages sur un terrain ou dans l'intérieur sont présumés faits par le propriétaire à ses frais et lui appartenir, si le contraire n'est prouvé ; sans préjudice de la propriété qu'un tiers pourrait avoir acquise ou pourrait acquérir par prescription soit d'un souterrain sous le bâtiment d'autrui, soit de toute autre partie du bâtiment $»$. 
que la surface des terrains, c'est les méconnaitre dans leur nature, c'est professer la doctrine des tyrans $»^{31}$, car " mal nommer un objet, c'est ajouter au malheur de ce monde $»^{32}$. Il apparait, au surplus, que le sol est moins un objet de droit lambda que l'objet préférentiel et majeur du droit de propriété telle qu'entendue par le Code civil de 1804 ; il en est la matrice originaire. Le sol, en effet, est le support central d'expression de la propriété. Construit autour du pouvoir qu'est la propriété, le Code civil est traversé par son expression. Pour autant, on l'a dit, le Code ne fait pas explicitement du sol per se un élément central, affirmé en tant que tel, alors que la propriété du sol en est la principale illustration.

\section{B. LE SOL, SUPPORT STRUCTURANT LA PROPRIÉTÉ}

Le sol ne paraît visé par le Code civil que comme expression d'une propriété préexistante : que ce soit l'accession ${ }^{33}$ de l'article 554, où la propriété du sol vient "agréger » celle des constructions qui sont dessus, la mitoyenneté des articles 660 et 661 qui posent le régime d'une construction séparant deux fonds distingués par le sol, l'article 1601-3 relatif au sort d'une construction future supposant le sol soit déjà approprié (encore pour les articles 1792 et 1793, la question se posant ici sur la responsabilité), et, enfin, l'article 1245-2 qui énonce indirectement le sol en se référant à des "produits $d u$ sol ». Dans chacun de ces cas, le Code civil vise le sol car il présuppose la propriété : ce sont donc les conséquences de la propriété du sol qui sont, en ces cas, traitées ; rien de plus. Dès lors, de deux choses l'une : soit le Code civil entend régir la propriété en structurant la propriété immobilière par la propriété du sol, soit, à l'inverse, la propriété, telle que conçue par les rédacteurs du Code, doit être regardée comme une matrice universelle dont l'objet demeure indifférent. La réponse se trouve certainement entre les deux : si l'enjeu primaire de la propriété a certainement été l'appropriation des terres, la propriété en tant que notion juridique doit être analysée comme une façon de structurer le droit, dont le sol constituait le principal support, sans en être le support exclusif.

31. A.P. XXIV, discours de Heurtault-Lamerville, député du Cher, p. 240 ; discours de Saint-Martin, p. 237 : cité par Poumarède J., De la difficulté de penser la propriété (17891793), in Propriété et Révolution, éd. CNRS, Toulouse 1, 1990, p. 33.

32. Camus A., Euvres complètes, T. I, La Pléiade, p. 908.

33. L'accession s'entend comme une conséquence de la propriété qui permet au propriétaire d'acquérir les accessoires que produit sa chose ou qui s'unissent ou s'incorporent à elle. 
Les codificateurs se posaient directement la question des liens entre l'appropriation du sol et la propriété, d'autant plus qu'ils en établissaient un rapport à la liberté : « La vraie propriété naît du partage des terres réalisé lorsque les hommes se lient par le pacte social qui fonde la société $\gg{ }^{34}$. La propriété du sol fonderait ainsi le socle même de la société. Rousseau ne disait-il pas la même chose lorsqu'il écrivait que « le premier qui, ayant enclos un terrain, s'avisa de dire : Ceci est à moi, et trouva des gens assez simples pour le croire, fut le vrai fondateur de la société civile. Que de crimes, de guerres, de meurtres, que de misères et d'horreurs n'eût point épargnés au genre humain celui qui, arrachant les pieux ou comblant le fossé, eût crié à ses semblables: Gardez-vous d'écouter cet imposteur ; vous êtes perdus, si vous oubliez que les fruits sont à tous, et que la terre n'est à personne $»^{35}$ ? «Dans le jeu d'une réflexion philosophique, la notion de propriété se comprenait comme une valeur rattachée en propre à l'homme, à ses activités créatrices, comme, par la suite, à son travail $»^{36}$. L'idée restera : «Le droit de propriété est celui qui appartient à tout citoyen de jouir à son gré de ses biens et de ses revenus, du fruit de son travail et de son industrie $\gg{ }^{37}$. Aussi le travail du sol a-t-il pu justifier son appropriation. Cependant, l'appropriation des fruits de celui-ci aurait pu également suffire, comme le proposait en son temps, par exemple, Pierre Joseph Proudhon ${ }^{38}$.

Mais cette explication ne suffit pas. Ou, plus précisément, cette explication ne suffit plus à expliquer l'étendue de la propriété du sol. Peut-être faut-il alors rechercher du côté de la qualité du sol, objet de notre travail, mais pas de celle qui lui est inhérente ; serait plutôt en cause celle qui lui a été conférée. La propriété du sol, en effet, permet de maintenir, sur une surface, une forme de pérennité par son caractère perpétuel ${ }^{39}$. Elle permet donc, par l'objet auquel elle s'applique, non seulement, de s'approprier les fruits qui en sont issus (appropriation classique), mais aussi, par la pérennité qu'elle instaure dans le droit, de s'approprier une qualité au sol conférée

\footnotetext{
34. Poumarède J., De la difficulté de penser la propriété (1789-1793), précité.

35. Rousseau J.J., Discours sur l'origine et les fondements de l'inégalité parmi les hommes, Deuxième partie, 1754.

36. Koubi G., De l'article 2 à l'article 17 de la Déclaration de 1789 : la brèche dans le discours révolutionnaire, in Propriété et Révolution, précité, p. 74.

37. Jaures J., Histoire socialiste de la Révolution, rééd. Soboul, T. 2, p. 469.

38. Proudhon P.J., Qu'est-ce que la propriété ?, op. cit. ; Proudhon P.J., Théorie de la propriété, op. cit.

39. Art. $2227 \mathrm{du}$ Code civil : «Le droit de propriété est imprescriptible ».
} 
par le travail régulier sur celui-ci (appropriation plus moderne). Autrement dit, la qualité est saisie juridiquement tout autant d'un point de vue subjectif, c'est-à-dire en tant que conséquence de l'appropriation du sol par un sujet, que d'un point de vue objectif, c'est-à-dire en vertu de composants et de processus biologiques. La qualité du sol demeure donc accessoire du sol en tant qu'objet de propriété mais, voilà la particularité, elle reste d'une part « affectée " par l'effet du travail de son propriétaire et, d'autre part, soumise à l'absolutisme de sa volonté. Elle devient, autrement dit, un objet de propriété en elle-même.

\section{LA PROTECTION DE LA QUALITÉ DU SOL PAR LA PROPRIÉTÉ}

Si la propriété est un droit absolu ${ }^{40}$, elle apparait conséquemment comme l'un des mécanismes de protection le plus efficace car tout autant absolu. Mais cette absoluité est à double tranchant s'agissant de la qualité des sols : elle est la cause de l'absorption par la propriété du sol de sa qualité (A), mais pourrait également être le moyen de sa protection par une autonomisation de l'objet (B).

\section{A. LA QUALITÉ ABSORBÉE PAR LA PROPRIÉTÉ DU SOL}

« La protection juridique de la qualité des sols n'est pas à la hauteur de son rôle vital. Cette situation serait liée au statut particulier de la ressource. Alors que l'on a pu s'atteler sans difficulté à la protection d'autres ressources naturelles telles que l'air, l'eau ou la biodiversité, la prise en compte des atteintes au sol reste encore largement insuffisante... Cela n'est guère surprenant. L'air et l'eau sont protégés, car ils sont juridiquement à l'usage de tous, alors que le sol, en tant qu'immeuble, est objet de propriété ; sa protection ne concerne donc que son propriétaire ${ }^{41}$. Autrement dit, puisque le sol est protégé par l'intermédiaire de son propriétaire, le droit civil ne connait pas d'une protection générale et spécifique de la qualité des sols. Il n'en reste pas moins que certaines dispositions spécifiques relatives à la qualité du sol peuvent être retrouvées. En ce sens, l'article L. 411-27 du Code rural et de la pêche maritime,

40. Comme l'énonce l'art. 544 du Code civil.

41. Collard Dutilleul F. (dir.), Dictionnaire juridique de la sécurité alimentaire dans le monde, Larcier, 2013, rubrique Sols, P. Steichen. 
en énonçant que «le fait que le preneur applique sur les terres prises à bail des pratiques ayant pour objet la préservation [...] de la qualité des sols », ne manque pas d'intérêt ${ }^{42}$. Mais il ne s'agit que de dispositions aux champs d'application très ponctuels, pour ne pas dire très isolés. Force est donc de constater que toute référence à la qualité des sols est absente au sein du Code civil.

Cette absence du Code civil ne doit cependant pas surprendre, et ce, pour au moins deux grandes types de justifications. D'une part, la qualité du sol n'est, on l'a dit, qu'un attribut du bien approprié, rien de plus. Dès lors, elle se confond avec l'utilité comme le démontre M. Beaussonie ${ }^{43}$. Par exemple, le défaut de qualité d'un sol ne motivera aucune appropriation en raison de son absence d'utilité ${ }^{44}$. Même si cette hypothèse est de moins en moins vraisemblable de nos jours, tant les tensions foncières sont devenues fortes ${ }^{45}$. D'autre part, la propriété appréhende une sphère de pouvoir qu'est l'appropriation. La qualité du sol est ici saisie, en tant que telle, parce qu'elle n'est qu'une conséquence de cette sphère d'appropriation. La qualité du sol peut très bien avoir motivé l'appropriation du bien. Mais elle devient alors, de façon indifférenciée donc non apparente, la chose du propriétaire, autant que le sol lui-même. En ce cas, la qualité du sol sera considérée comme issue d'une chose frugifère - le sol - dont il reviendra au propriétaire de décider de la conserver ou non.

L'hypothèse est classique et peut s'illustrer aisément : le propriétaire d'une forêt peut tout autant décider d'y recueillir divers éléments que la chose frugifère produit, tout comme il peut décider de la raser afin d'en récupérer le produit qu'est le bois ${ }^{46}$. Appliquée au

42. V. Hermon C., La protection du sol en droit, supra, et Krajeski D., Travail du sol, services écosystémiques, et bail rural, infra.

43. Beaussonie G., op. cit., supra.

44. Ce fut le cas pendant un temps des déserts notamment, dont l'absence d'utilité n'impliquait pas d'utilité à l'appropriation. C'est partiellement encore le cas pour les res communes que sont l'eau et l'air.

45. Sur le foncier agricole par ex., V. not. Cavailhes J., Mesrine A., Rouquette C., Le foncier agricole : une ressource sous tensions, Economie et Statistique, n ${ }^{\circ} 444-445,2011$.

46. Sous réserve de certaines autorisations administratives et éventuelles obligations de reconstitution, relatives à la taille de la forêt en question et de son éventuel classement. En ce sens, le Règlement du 20 octobre 2010, n 995/2010, de 1'Union Européenne établissant les obligations des opérateurs qui mettent du bois et des produits dérivés sur le marché vient encadrer la coupe du bois et sa commercialisation ; complété par le Règlement Délégué de la Commission Européenne n 363/2012 du 23 février 2012 relatif aux règles de procédure concernant la reconnaissance et le retrait de la reconnaissance 
sol, cette logique ne diffère pas : la propriété d'un sol donne droit à son titulaire de conférer ou maintenir la qualité de cet objet de droit tout autant que de la consommer. En ce sens, le propriétaire d'un sol pourra décider d'exploiter et éventuellement maintenir la qualité de son sol, tout comme il pourra l'épuiser. Aussi pourra-t-il anéantir la qualité d'un sol par une exploitation agricole intensive, tout comme il pourra maintenir, voire augmenter cette qualité par la même activité agricole mais selon d'autres procédés ${ }^{47}$.

«A priori, aucune particularité écologique n'est recherchée pour la répartition de la propriété du sol qui n'est que son support abstrait $\gg^{48}$. Si la propriété du sol annexe sa qualité, c'est peut-être également parce que, on l'a dit, le sol n'est appréhendé que comme moyen, c'est-à-dire que le sol n'apparait, en ce cas, que comme le support d'expression de la propriété. Autrement dit, c'est parce que la propriété du sol permet la pérennisation de la propriété du dessus, notamment des constructions qui y seront faites, que le sol est l'objet préalable de la propriété. En ce sens, le sol n'est que le moyen d' " être propriétaire », et conséquemment, la propriété s'intéresse moins au sol per se - encore moins à sa qualité - qu'à l'appropriation de sa surface, c'est-à-dire au sol en tant que support. Dès lors, puisqu'on ne s'intéresse donc pas vraiment au sol, pourquoi s'intéresser à sa qualité ?

En effet, « le droit de propriété publique ou privée trouve son fondement dans la propriété du sol $»^{49}$, et non dans sa qualité. Cependant, il reste que cette qualité du sol conditionne, au moins pour partie, la volonté d'appropriation. Par exemple, l'acquisition d'un terrain en vue d'y établir une construction visera moins la qualité d'un sol que son emplacement géographique ; a contrario, si l'objectif est d'y installer une activité agricole, il parait évident que le propriétaire s'attachera bien plus aux qualités potentielles

des organisations de contrôle conformément au règlement, et le Règlement d'exécution de la Commission $\mathrm{n}^{\circ}$ 607/2012 du 6 juillet 2012 sur les modalités d'application relatives au système de diligence, ainsi qu'à la fréquence et à la nature des contrôles à effectuer auprès des organisations de contrôle conformément au règlement.

47. Cf. Chabert A., Expression combinée des services écosystémiques en systèmes de production agricole conventionnels et innovants : étude des déterminants agroécologiques de gestion du sol, des intrants et du paysage, Th. Toulouse, INPT, 2017.

48. Desrousseaux M., La protection juridique de la qualité des sols, LGDJ, Biblio. de Dr. de l'Urba. et de l'Env., T. 13, 2016, n² 28, p. 11.

49. Idem. 
de ce sol, encore que cette qualité puisse dépendre de son travail. La qualité du sol n'est pas l'objet de droit appropriable. Le seul objet de droit ici présent reste le sol, sa qualité y étant rattachée au mieux en tant qu'essence du bien et, a minima, en tant qu'accessoire par la propriété du dessous. Aussi, en matière de droit de l'urbanisme, la qualité du sol viendra-t-elle parfois être prise en compte mais seulement en ce qu'elle est un moyen de catégorisation générale, permettant ou excluant certaines utilisations des sols ${ }^{50}$. Distinguer le sol de sa qualité apparait alors quasi-chimérique tant l'un et l'autre sont inexorablement et consubstantiellement liés. Toutefois, Madame Desrousseaux relève que « le droit de propriété reconnait deux types de sols : l'un constitué de matière vivante l'autre servant de support aux ouvrages, mais régis par un seul et même régime $»^{51}$. Si une telle unité de régime ne fait aucun doute, cette unité se justifie à notre sens par l'unité de notion : il n'existe qu'une seule et unique propriété. Le propos de cette autrice n'en reste pas moins perspicace, une partie de la doctrine proposant en ce sens une protection particulière du vivant ${ }^{52}$, ces propositions ayant reçu un certain écho chez le législateur quant aux animaux ${ }^{53}$.

\section{B. LA QUALITÉ DU SOL, OBJET DE PROPRIÉTÉ AUTONOME?}

Si notre conception de la propriété semble écarter, à tout le moins limiter, toute protection de la qualité des sols, si ce n'est par l'intermédiaire de leur propriétaire, il n'en reste pas moins que des propositions doctrinales émergent afin d'assurer une telle protection. En ce sens, le doyen Duguit ne proposait-il pas au début du $X^{\text {ème }}$ siècle d'affirmer la fonction sociale de la propriété ${ }^{54}$ ?

50. Le Code de l'urbanisme distingue par exemple les espaces agricoles et naturels périurbains (art. L. 143-1 à L. 143-6) des espaces boisés (art. L. 130-1 à L. 130-6) ou encore des zones urbaines, dont les plans locaux d'urbanisme sont chargés de préciser les affectations (art. L. 123-1 à L. 123-20).

51. Desrousseaux M., La protection juridique de la qualité des sols, op. cit., $\mathrm{n}^{\circ} 27, \mathrm{p} .11$.

52. Neyret L., Atteintes au vivant et responsabilité civile, LGDJ, Biblio. de dr. priv., T.468, 2006.

53. L'art. 515-14 du Code civil introduit par la loi du 16 février 2015 n² 2015-177, énonce : «Les animaux sont des êtres vivants doués de sensibilité. Sous réserve des lois qui les protègent, les animaux sont soumis au régime des biens ».

54. V. not. Duguit L., Les transformations générales du droit privé depuis le Code Napoléon, Paris, Libr. Félix Alcan, 1912. V. ég. du même auteur : Duguit L., Le droit social, le droit individuel et la transformation de l'Etat, Paris, Libr. Félix Alcan, $2^{\mathrm{e}}$ éd. 1911. Sur la doctrine de Duguit L.: V. not. Boccon-Gibod T., Duguit, et après ? Droit, propriété et rapports sociaux, RIDE, 2014, p. 285 et s ; Melleray F. (Dir.), Autour de Léon Duguit, 
Partant du constat que le système juridique tend à se fonder sur une " conception essentiellement socialiste $\|^{55}$ - entendue de la socialisation du droit ${ }^{56}$ - ainsi qu'une « conception métaphysique de droit subjectif $»^{57}$, Duguit postule que l'être humain « isolé et indépendant est une pure fiction [...]. L'homme est un être social ; il ne peut vivre qu'en société ; il a toujours vécu en société ${ }^{58}$. Dès lors, « l'homme n'a pas de droits ; la collectivité n'en a pas l'avantage. Mais tout individu a dans la société une certaine fonction à remplir, une certaine besogne à exécuter $»^{59}$. Illustrant son propos par la propriété, il affirme que « la propriété n'est pas un droit, elle est une fonction sociale $\|^{60} \mathrm{c}$ 'est-à-dire que si le propriétaire « ne la remplit pas ou mal, [...] l'intervention des gouvernants est légitime pour le contraindre » à celle-ci ${ }^{61}$. Si cette proposition est séduisante notamment en ce qu'elle pourrait obliger les propriétaires fonciers à garantir une certaine qualité de leur sol au titre de la réalisation de la fonction sociale de la propriété, elle n'en reste pas moins éloignée de l'interprétation contemporaine de la propriété qui soumet à la propriété la plupart des autres droits, plus que ce qu'ils ne la soumettent ${ }^{62}$. Plus récemment, $M$. Ost développait l'idée d'une 《 transpropriation ${ }^{63}$ visant à considérer que le patrimoine commun de l'humanité implique la cohabitation sur un même objet de droit de plusieurs propriétaires : d'une part se retrouve le propriétaire tel qu'entendu classiquement, celui qui est « propriétaire » de la chose ;

Bruxelles, Bruylant, 2011. Pour une autre approche : Renard G. et Trotabas L., La fonction sociale de la propriété, Paris, S., 1930. V. ég. Millet L., Contribution à l'étude des fonctions sociale et écologique du droit de propriété : enquête sur le caractère sacré de ce droit énoncé dans la Déclaration des droits de l'homme et du citoyen du 26 août 1789, Th. Paris I, 2015 ; Fabre-Magnan M., Propriété, patrimoine et lien social, RTD civ., 1997, p. 583.

55. Duguit L., Les transformations générales du droit privé depuis le Code Napoléon, op. cit., p. 8.

56. Duguit L.se réfère ici à not. : Charmont J., La socialisation $d u$ droit, Revue de métaphysique et de morale, 1903, p. 403 ; et A. Mater, Le socialisme juridique, Revue socialiste, XL, 1904, p. 9 et s.

57. Duguit L., Les transformations générales du droit privé depuis le Code Napoléon, op. cit., p. 9.

58. Ibid.p. 18.

59. Ibid. p. 19.

60. Ibid. p. 21.

61. Idem.

62. Le caractère absolu de la propriété énoncé à l'art. 544 du Code civil implique que toute limitation de ce droit soit per se limitée, en témoigne la limite " aux lois et règlements » prévu par ce même texte, ou encore «juste et préalable indemnité » pour " cause d'utilité publique » de l'art. 545 du même Code.

63. Ost F., La nature hors la loi, L'écologie à l'épreuve du droit, éd. La découverte, 2003, spéc. p. 338. 
d'autre part, certaines utilités seraient détachées du propriétaire au bénéfice de la collectivité : « il en résulte que le maitre et propriétaire de l'espace ou de la ressource ne dispose pas de la même intensité d'emprise sur chacun des aspects de son bien ; certains usages restent souverains (pouvant aller jusqu'au droit de détruire), d'autres sont désormais contrôlés ou interdits $»^{64}$. Appliqué aux sols, la transpropriation viserait à considérer la qualité du sol comme relevant du patrimoine commun de la Nation par exemple, tout en laissant la propriété de ce même sol à son actuel titulaire. La récente loi sur la biodiversité 65 reconnait en ce sens que « la protection des sols d'intérêt général, en les intégrant au patrimoine commun de la nation dans le Code de l'environnement ». Ici aussi, le modèle apparait intéressant mais supposerait de déterminer un véritable régime juridique, surtout préalablement, de consacrer l'efficience des patrimoines communs, considérés pour l'instant comme simplement déclaratoires ${ }^{66}$. Enfin, un courant doctrinal récent vise à affirmer l'existence des $"$ communs $\|{ }^{67}$ supposant une véritable « révolution juridique ${ }^{68}$ par la proclamation des biens communs. Il reste que toutes ces propositions consistent soit en une limitation de la propriété, soit en une affirmation d'un autre objet de propriété.

Mais certaines dispositions mériteraient peut-être de connaitre un autre destin et pourraient déjà être des solutions. Nous l'évoquions plus tôt, le Code rural prévoit une éventuelle prise en compte de la qualité des sols ${ }^{69}$. Cette disposition, appliquée au bail rural, pourrait permettre de donner un cadre juridique de protection de la qualité du sol. En effet, l'article L. 411-69 du même Code ${ }^{70}$ prévoit qu'à la fin

64. Ibid. p. 339.

65. Loi n ${ }^{\circ} 2016-1087$ du 8 août 2016 pour la reconquête de la biodiversité, de la nature et des paysages.

66. V. Savarit I., Le patrimoine commun de la nation, déclaration de principe ou notion juridique à part entière ?, RFDA, 1998, p. 305 ; Groulier C., Quelle effectivité juridique pour le concept de patrimoine commun?, AJDA, 2005, p. 1034 ; Del Rey M.-J., La notion controversée de patrimoine commun, D. 2006, p. 388 ;

67. V. not. Ostrom E., Gouvernance des biens communs, Pour une nouvelle approche des ressources naturelles, éd. De Boeck, Bruxelles, 2010, 1éd. ; Parance B. et de Saint Victor J. (dir.), Repenser les biens communs, CNRS éd., Paris, 2014 ; Coriat B., Le retour des communs, La crise de l'idéologie propriétaire, éd. Les liens Qui Libèrent, 2015.

68. Parance B. et de Saint Victor J. (dir.), Repenser les biens communs, op. cit., p. 9.

69. Art. L. 411-27 C. rur.

70. Art. L. 411-69 C. rur. : "Le preneur qui a, par son travail ou par ses investissements, apporté des améliorations au fonds loué a droit, à l'expiration du bail, à une indemnité due par le bailleur, quelle que soit la cause qui a mis fin au bail ». 
du bail rural, le preneur doit restituer le fonds dans l'état dans lequel il l'a trouvé ${ }^{71}$, l'état initial étant défini par un état des lieux établi à la prise à bail ${ }^{72}$. En déterminant à la conclusion du bail le niveau de qualité du sol, nous pourrions aisément imaginer ${ }^{73}$ une obligation de restitution du même niveau de qualité qui, considérée objectivement, profiterait au fonds lui-même ${ }^{74}$.

Il pourrait en aller de même avec le bail du Code civil : le preneur à bail s'oblige en effet à restituer, en fin de bail, «la chose telle qu'il l'a reçue $"{ }^{75}$. Cette disposition vise évidemment à protéger le propriétaire de la chose louée en ce que le locataire respecte la destination de la chose louée, mais également en ce qu'il la restitue telle qu'elle lui a été délivrée ; à défaut, il en est responsable ${ }^{76}$. Sans excès d'imagination, il n'est pas difficile de considérer que la qualité du sol de la chose louée, par exemple le jardin d'une maison, puisse se voir appliquer de telles dispositions. Il conviendrait en ce cas de déterminer au sein du bail le niveau de qualité du sol à l'entrée des lieux. Il est cependant permis de s'interroger sur la destination d'une telle protection : protègerait-on le propriétaire ou la chose elle-même ? Nous pensons que le premier conserverait le primat sur l'autre. En effet, une telle clause, si elle bénéficierait au sol comme à son propriétaire, reste pour autant sujette à l'unique volonté de ce dernier, tant dans l'insertion au sein du bail, que dans le maintien de

71. V. Krajeski D., Droit rural, 2e éd., Lextenso Ed., 2016, spéc. n 187, p. 183.

72. V. art. 1730 C. civ. : "S'il a été fait un état des lieux entre le bailleur et le preneur, celui-ci doit rendre la chose telle qu'il l'a reçue, suivant cet état, excepté ce qui a péri ou a été dégradé par vétusté ou force majeure ». Nous soulignons que cette disposition est applicable aux baux ruraux mais aussi aux «baux des maisons ».

73. Il conviendrait certainement de s'interroger si cette obligation, qui semble bénéficier initialement au propriétaire, ne bénéficierait-elle alors pas au fonds lui-même?

74. Une difficulté textuelle devrait ici être levée par le législateur : toutes les améliorations du fonds n'étant pas susceptibles d'être l'objet d'une indemnisation, l'article L. 411$69 \mathrm{du}$ Code rural visant les bâtiments indispensables à l'exploitation du bien loué ou l'habitation du preneur, il est à craindre que l'amélioration de la qualité du sol ne le soit pas nécessairement. La même difficulté apparait avec l'article L. 411-29 du même Code qui exclut le recours à l'indemnisation : V. Krajeski D., Travail du sol, services écosystémiques, et bail rural, infra.

75. Art. 1730 C. civ. : «S'il a été fait un état des lieux entre le bailleur et le preneur, celuici doit rendre la chose telle qu'il l'a reçue, suivant cet état, excepté ce qui a péri ou a été dégradé par vétusté ou force majeure ».

76. Art. 1732 C. civ. : "Il répond des dégradations ou des pertes qui arrivent pendant sa jouissance, à moins qu'il ne prouve qu'elles ont eu lieu sans sa faute ». 
sa portée ultérieurement au bail. Seule une intervention législative saurait perpétuer son esprit ${ }^{77}$.

«Propriété des terres, mère de tout $\rangle^{78}$ a pu écrire Montesquieu. Difficile d'affirmer le contraire, en l'espèce, tant la propriété semble s'être structurée en lien avec la terre. Difficile également de distinguer le sol de sa qualité, l'un et l'autre étant consubstantiellement liés, comme le démontre le régime de la propriété ${ }^{79}$. Il apparait, en ce sens, que le sol en tant que support d'une assiette de propriété, est l'objet de droit qui contient sa qualité. Nous pensons, dès lors, que la propriété ne peut faire qu'obstacle à l'appréhension juridique de la qualité des sols, en ce sens que, on l'a vu, cette dernière ne peut se distinguer du sol; le sol est tout autant un objet de droit que le support de ce droit. Ne restent alors que deux solutions : soit faire de la qualité des sols un objet de droit à part entière, c'est-à-dire détacher une utilité du sol pour en faire un bien en soi $^{80}$; soit limiter la propriété par une servitude de protection de certaines qualités des sols, tel qu'il en existe aujourd'hui en matière minière ou encore en matière d'aqueduc ${ }^{81}$. À défaut, il est inutile, au sens du droit civil du moins, de parler de « qualité du sol».

77. Encore qu'il s'agisse d'une limitation de la propriété, constitutionnellement protégée. Sur la limitation acceptée, V. Cons. const., 16 janv. 1982, n 81-132 DC, D. 1983. 169, note Hamon L., JCP 1982. II. 19788, note Nguyen Vinh et Franck, Gaz. Pal. 1982. 1. 67, note Piédelièvre et Dupichot, Rev. crit. DIP 1982. 349, note Bischoff; contra Cons. const., 7 déc. 2000, n 2000-436 DC, AJDA 2001. 18, note Schoettl J.-E.; D. 2001. 1840, obs. Favoreu L. 78. Montesquieu, Pensées, ${ }^{\circ} 1839$.

79. $C f$. art. 552 C. civ.

80. Par ex. sur le numéro de carte de crédit distinct de la carte elle-même : Crim., 14 nov. $2000, \mathrm{n}^{\circ} 99-84522$.

81. V. art. L. $152-17$ C. rur.. 


\section{PROPOSITIONS POUR UNE PROTECTION DES SOLS EN DROIT}



L'INTÉGRATION DE LA NOTION DE SERVICE ÉCOSYSTÉMIQUE EN DROIT 



\title{
Brève histoire de l'intégration de la notion de service écosystémique en droit
}

\author{
Isabelle DOUSSAN \\ Directrice de Recherche INRA \\ GREDEG UMR 7321 CNRS \\ Université Côte d'Azur, 06560, Valbonne, France
}

\section{INTRODUCTION : DES ORIGINES SCIENTIFIQUES DES SERVICES ÉCOSYSTÉMIQUES À LEUR INSTITUTIONNALI- SATION}

Dès les années soixante-dix, notamment à l'occasion de la préparation de la Conférence de l'ONU qui s'est tenue à Stockholm en 1972, certains travaux d'écologues américains mettent en avant la notion de services écosystémiques. Mais c'est surtout à partir des années quatre-vingt que cette notion a été développée et a fait l'objet d'un nombre de publications croissant. La notion est dès ses origines marquée par un double contexte : d'une part, l'intégration dans les travaux en sciences de l'écologie des dimensions systémiques et dynamiques des processus écologiques et, d'autre part, la volonté de certains scientifiques d'alerter sur « la possible atteinte des limites des capacités de charge de la planète $»^{1}$ soumise au développement des activités humaines. Emerge alors l'idée que l'évaluation des « services rendus par la nature » peut renforcer la prise de conscience publique de l'importance du bon fonctionnement des écosystèmes pour la vie humaine. Dans cette veine, on trouve des travaux d'écologues proposant une analyse économique des fonctions

1. Mongruel R., Méral P., Doussan I., Levrel H., 2016, L'institutionnalisation de l'approche par les services écosystémiques : dimensions scientifiques, politiques et juridiques. In : Valeurs de la biodiversité et services écosystémiques. Perspectives interdisciplinaires (Roche P., Geijzendorffer I., Levrel H., Maris V., eds), collection Up date Sciences technologies, éditions Quae, Versailles, 191-216, p. 192 
écologiques considérées comme essentielles pour le bien-être humain et des travaux d'économistes reposant sur la notion de " capital naturel » comme élément contribuant au développement des sociétés humaines. Un article en particulier fait date, celui de Costanza et al. publié en 1997 dans la revue Nature, où l'équipe de chercheurs propose une évaluation monétaire des services écosystémiques et du capital naturel à l'échelle mondiale².

Puis ce sont des rapports publiés par des ONG internationales qui reprennent ces travaux et l'approche de la nature via les services écosystémiques pénètre ainsi les sphères politiques. «Cette approche est mobilisée au service d'un objectif clairement affiché : dépasser la segmentation des problématiques sectorielles liées à l'existence de conventions internationales thématiques sur la diversité biologique, la désertification, le changement climatique, etc..., et proposer une vision globale et "écosystémique" des enjeux environnementaux $»^{3}$.

Un événement déterminant pour l'institutionnalisation du concept de services écosystémiques est très certainement la publication en 2005 du Millenium Ecosystem Assessment (MEA), étude de très grande ampleur portée par l'ONU et soutenue par plusieurs grandes organisations internationales ${ }^{4}$. L'étude qui mobilise plus de mille chercheurs pendant plus de trois ans sera largement diffusée. Selon la présentation même du rapport de l'ONU, « le fait que cette évaluation s'attarde aux fonctions écosystémiques et à leur lien avec le bien-être humain et les besoins en développement est unique en son genre. En examinant l'environnement selon le cadre des fonctions écosystémiques, il est bien plus facile de déterminer comment les changements écosystémiques influent sur le bien-être humain et de présenter de l'information sous une forme que les décideurs peuvent comparer à d'autres renseignements sociaux et économiques ». Le MEA propose alors un cadre d'analyse et une typologie des services écosystémiques classés en quatre

2. Costanza R., d'Arge R., De Groot R., Farber S., Grasso M., Hannon B., Limburg K., Naeem S., O'neill R., Paruelo J., Raskin R-G., Sutton P., Van den Belt M., 1997, The value of the world's ecosystem services and natural capital, Nature, 387, 253-260.

3. Mongruel R., Méral P., Doussan I., Levrel H., 2016, L'institutionnalisation de l'approche par les services écosystémiques : dimensions scientifiques, politiques et juridiques, précité, p. 195.

4. http://www.millenniumassessment.org/fr/ 
grandes catégories ${ }^{5}$. Si par la suite cette typologie sera complétée, critiquée, modifiée par de nombreux travaux, ce rapport contribue incontestablement à asseoir l'approche par services écosystémiques et à alerter sur leur dégradation. " Au-delà d'un état des lieux de la biodiversité globale, cette expertise internationale a surtout permis d'articuler et de communiquer le concept de service écosystémique et de fournir une méthodologie applicable de façon générique pour quantifier les conséquences des changements de biodiversité sur les écosystèmes et sur différentes composantes du bien-être humain. Depuis, ce travail a stimulé une profusion de travaux de recherche sur les services écologiques, que ce soit des développements méthodologiques, des études de cas pour des systèmes ou services particuliers (par exemple la pollinisation), ou de nouvelles avancées sur les mécanismes impliqués dans les relations entre biodiversité et services écologiques, ainsi que sur les mécanismes d'évaluation socioéconomique de la biodiversité et des services écologiques $\|^{6}$. On observe ainsi que « la montée en puissance du concept de service écosystémique (...) est le résultat d'une co-construction entre science et politique à l'échelle internationale (...) l'institutionnalisation de cette approche n'a donc pas été un processus distinct de celui de sa construction scientifique $»^{7}$.

Du fait même sans doute de cette construction simultanée, les services écosystémiques pénètrent le champ des politiques publiques et du droit sans être stabilisés scientifiquement, traduisant ainsi une approche renouvelée de l'environnement.

\footnotetext{
5. A savoir : $1^{\circ}$ - les services d'approvisionnement (également appelés parfois « services de prélèvement »). Ce sont les produits que procurent les écosystèmes, tels que les ressources génétiques, la nourriture et la fibre, ainsi que l'eau douce. $2^{\circ}$ - Les services de régulation. Ce sont les bienfaits qui découlent de la régulation des processus liés aux écosystèmes, tels que la régulation du climat, de l'eau et de certaines maladies humaines. $3^{\circ}$ - Les services culturels. Ce sont les bienfaits non matériels que procurent les écosystèmes à travers l'enrichissement spirituel, le développement cognitif, la réflexion, les loisirs et l'expérience esthétique, tels que les systèmes de savoir, les relations sociales et les valeurs esthétiques. $4^{\circ}$ - Les services de soutien. Ce sont les services nécessaires à la production de tous les autres services fournis par les écosystèmes. Ils comprennent la production de biomasse, la production d'oxygène atmosphérique, la formation et la rétention des sols, le cycle des éléments nutritifs, le cycle de l'eau et l'offre d'habitats.

6. Le Roux X., Barbault R., Baudry J., Burel F., Doussan I., Garnier E., Herzog F., Lavorel S., Lifran R., Roger-Estrade J., Sarthou J-P., Trommetter M.(eds), 2008. Agriculture et biodiversité. Valoriser les synergies. Expertise scientifique collective, INRA, France, 116 p. 7. Mongruel R., Méral P., Doussan I., Levrel H., 2016, L'institutionnalisation de l'approche par les services écosystémiques : dimensions scientifiques, politiques et juridiques, précité, p. 197.
} 


\section{L'ENTRÉE DES SERVICES ÉCOSYSTÉMIQUES DANS LE CHAMP DU DROIT ET DES POLITIQUES PUBLIQUES}

C'est à partir du MEA que les services écosystémiques investissent formellement le champ des politiques environnementales et du droit. Le concept ne figure pas, par exemple, dans la Convention sur la diversité biologique adoptée lors du Sommet de la Terre à Rio de Janeiro en 1992. Dans les textes de l'Union européenne, il est absent de la communication "Stratégie communautaire en faveur de la diversité biologique $\rangle^{8}$ de 1998 et à peine implicite dans la communication «Plan d'action en faveur de la diversité biologique dans le domaine de l'agriculture $»^{9}$ de 2001. En revanche, il est au cœur de deux communications de 2006 et 2007. L'une, portant sur la biodiversité, est intitulée « Enrayer la diminution de la biodiversité à l'horizon 2010 et au-delà. Préserver les services écosystémiques pour le bien-être humain $\rangle^{10}$, l'autre constitue l'examen à mi-parcours du sixième programme d'action communautaire pour l'environnement ${ }^{11}$. Quant aux textes communautaires dont le respect s'impose aux Etats membres, on remarquera que dans la directive 2000/60, dite directive cadre sur l'eau ${ }^{12}$, la notion de service des écosystèmes, en l'espèce aquatiques, n'apparaît pas. En revanche, la directive du 21 avril 2004 sur la responsabilité environnementale ${ }^{13} \mathrm{y}$ fait explicitement référence comme élément de définition du dommage environnemental, en employant le terme de « services écologiques ». Compte tenu de l'objectif du MEA qui est de mettre en avant les atteintes affectant les services écosystémiques, ce n'est d'ailleurs sans doute pas un hasard que son premier emploi en droit se fasse dans le cadre d'un régime de réparation des dommages à l'environnement.

On observe ensuite que des textes sectoriels s'appuient sur les services écosystémiques pour fonder des régimes de protection de certaines ressources. Notamment, en 2006, la proposition de directive sur les sols « définit un cadre pour la protection des sols

\footnotetext{
8. COM (1998)42, non publié.

9. COM (2001)162 final.

10. COM (2006)216 final.

11. COM (2007)225 final.

12. Directive 2000/60 du Parlement européen et du Conseil du 23 octobre 2000 établissant un cadre pour une politique communautaire dans le domaine de l'eau.

13. Directive 2004/35/CE du Parlement européen et du Conseil sur la responsabilité environnementale en ce qui concerne la prévention et la réparation des dommages environnementaux.
} 
et la préservation de leur capacité à remplir (...) des fonctions écologiques, économiques, sociales et culturelles » $\left(\text { art. } 1^{\mathrm{er}}\right)^{14}$. Les services figurent également au cœur de la directive-cadre Stratégie pour le milieu marin du 17 juin $2008^{15}$, puis du règlement du 22 octobre 2014 relatif à la prévention et à la gestion des espèces exotiques envahissantes ${ }^{16}$.

En droit français, les termes de « services écosystémiques » sont restés longtemps assez peu utilisés, même si ce type d'approche a fait incontestablement du chemin $(C f$. infra, M. Fèvre « Les "services écosystémiques", une notion fonctionnelle »). En 2008, la loi de transposition de la directive sur la responsabilité environnementale intègre les services écologiques, dans le cadre d'un régime de police administrative très spécifique. Inscrits à l'article L.161-1 I $4^{\circ}$ du Code de l'environnement, les services écologiques sont définis comme les fonctions assurées par les sols, les eaux et les espèces et habitats protégés, au bénéfice d'une de ces ressources naturelles ou au bénéfice du public. Leur définition semble ainsi permettre de les assimiler aux services écosystémiques. Ce sont bien ces derniers en revanche qui sont explicitement visés, en 2014, par la loi d'avenir pour l'agriculture, l'alimentation et la forêt ${ }^{17}$ à propos des « systèmes de production agroécologiques, dont le mode de production biologique, qui combinent performance économique, sociale (...), environnementale et sanitaire. Ces systèmes (...) sont fondés sur les interactions biologiques et l'utilisation des services écosystémiques et des potentiels offerts par les ressources naturelles (...) $\gg{ }^{18}$. Ce sont ces systèmes de production agricole que «les politiques publiques visent à promouvoir et à pérenniser ${ }^{19}$.

14. La définition de ces fonctions renvoie d'ailleurs davantage à celles de " services » traduisant ainsi une certaine confusion sémantique dans le champ juridique, perceptible également en droit français. Cf. supra $\mathrm{C}$. Hermon « La protection du sol en droit» et infra M. Fèvre «Les "services écosystémiques", une notion fonctionnelle».

15. Directive 2008/56/CE du Parlement Européen et du Conseil du 17 juin 2008 établissant un cadre d'action communautaire dans le domaine de la politique pour le milieu marin.

16. Règlement (UE) n ${ }^{\circ} 1143 / 2014$ du Parlement européen et du Conseil du 22 octobre 2014, relatif à la prévention et à la gestion de l'introduction et de la propagation des espèces exotiques envahissantes. Article $4.3 \mathrm{c}$ ) : «Les espèces exotiques envahissantes [...] sont, sur la base des preuves scientifiques disponibles, susceptibles d'avoir des effets néfastes importants sur la biodiversité ou les services écosystémiques associés [...] ».

17. Loi n ${ }^{\circ} 2014-1170 \mathrm{du} 13$ octobre 2014.

18. Art. L.1-II du Code rural et de la pêche maritime.

19. Art. L.1-II du Code rural et de la pêche maritime. 
Mais c'est sans aucun doute la loi pour la reconquête de la biodiversité, de la nature et des paysages du 8 août 2016 qui fait une plus grande place aux services écosystémiques. Dès l'exposé des motifs du projet de loi, il est indiqué que la biodiversité « force économique pour la France (...) assure des services qui contribuent aux activités humaines, dit services écosystémiques ». Dans la loi elle-même, «la sauvegarde des services» fournis par «les espaces, ressources et milieux naturels terrestres et marins, les sites, les paysages diurnes et nocturnes, la qualité de l'air, les êtres vivants et la biodiversité »" est déclarée «d'intérêt général $»^{20}$. Le principe d'action préventive et de correction, par priorité à la source, est également complété en ce qu'il implique désormais «d'éviter les atteintes à la biodiversité et aux services qu'elle fournit $»^{21}$. Enfin, les services sont introduits par la loi de 2016 dans le Code civil français, dont l'article 1247 reconnait le préjudice écologique et le définit comme « une atteinte non négligeable aux éléments ou aux fonctions des écosystèmes ou aux bénéfices collectifs tirés par l'homme de l'environnement. ", cette dernière expression visant incontestablement les services écosystémiques. On peut enfin ajouter le nouveau principe de complémentarité entre l'environnement et l'agriculture qui renvoie non plus aux services écosystémiques mais aux « services environnementaux » que peuvent fournir les activités agricoles, aquacoles et forestières en utilisant «les fonctions écologiques d'un écosystème pour restaurer, maintenir ou créer de la biodiversité $\gg{ }^{22}$.

La loi du 8 août 2016 est encore trop récente pour en mesurer les effets, mais il semble que l'on peut avancer que l'intégration dans les textes juridiques des services écosystémiques est susceptible de renouveler l'appréhension juridique de l'environnement.

\section{LES SERVICES ÉCOSYSTÉMIQUES ET L'APPRÉHENSION JURIDIQUE DE L'ENVIRONNEMENT}

Les éventuels effets du concept de services écosystémiques sur l'appréhension juridique de l'environnement sont à rechercher tant dans le terme de " service » que dans celui d'écosystème. C'est le premier qui a, semble-t-il, davantage retenu l'attention des auteurs. 
Les critiques principales qui sont formulées à l'encontre des services écosystémiques dénoncent l'approche utilitariste et anthropocentrée de l'environnement qu'ils traduisent ${ }^{23}$.

Pourtant, dans le champ du droit, on peut remarquer que les ressources naturelles ont été et sont encore appréhendées et protégées au regard de l'utilité qu'elles présentent pour l'homme. C'est le cas pour l'eau notamment, dont «la gestion équilibrée doit permettre en priorité de satisfaire les exigences de la santé, de la salubrité publique, de la sécurité civile et de l'alimentation en eau potable de la population. $\gg^{24}$ On peut également considérer que nombre d'espaces et espèces végétales et animales, la biodiversité « remarquable » ou « patrimoniale », sont protégés par le droit en raison des services culturels qu'ils offrent.

De ce point de vue, les services écosystémiques ne semblent pas de nature à modifier sensiblement l'approche juridique de l'environnement naturel. Ils pourraient même conforter la protection accordée par le droit, renforcer sa légitimité. Ils expriment en effet la dépendance forte des sociétés humaines aux écosystèmes et à leur bon fonctionnement, comme le reconnait la Constitution française selon laquelle « l'avenir et l'existence même de l'humanité sont indissociables de son milieu naturel $\gg^{25}$. Cette dépendance est si forte que préserver les écosystèmes et leurs services, c'est préserver les sociétés humaines et leurs activités. Si «cette dépendance a longtemps été considérée comme un obstacle à surmonter, les progrès scientifiques et techniques se faisant les garants de la domination des hommes sur le monde naturel $»^{26}$, le concept de services écosystémiques tend à montrer qu'elle est en grande part indépassable, inhérente à la vie sur terre.

Mais on sait aussi que le droit rend compte également, souvent de manière implicite, d'une valeur intrinsèque de la nature, indépendamment de l'utilité qu'elle présente pour les humains. En ce sens, le droit de l'environnement traduit incontestablement un élargissement

23. Cf. par exemple, Maris V., 2014. Nature à vendre. Les limites des services écosystémiques, ed. Quae, coll. Sciences en questions.

24. Art. L.211-1 II C. env.

25. Loi constitutionnelle $\mathrm{n}^{\circ} 2005-205 \mathrm{du} 1^{\mathrm{er}}$ mars 2005 relative à la Charte de l'environnement.

26. Maris V., op. cit., p. 23. 
des intérêts juridiquement protégés, les humains ne sont plus seuls dans le monde du droit. L'environnement y existe en effet comme un objet de protection en soi, un " centre d'intérêts $\|^{27}$ qui ne sont pas seulement humains. On peut ici citer la Charte mondiale de la nature adoptée par l'assemblée générale des Nations-Unies le 28 octobre 1982, dont le préambule déclare que « toute forme de vie est unique et mérite d'être respectée, quelle que soit son utilité pour l'homme ». Dix ans plus tard, dans le préambule de la convention sur la diversité biologique, les Etats se proclament « conscients de la valeur intrinsèque de la diversité biologique ». En conséquence, si l'environnement est protégé en droit en raison de son utilité pour l'homme, il l'est également per se.

Le risque pourrait être alors que le succès et le caractère opérationnel du concept de services écosystémiques agissent comme des rouleaux compresseurs et en viennent à «écraser 》 dans le champ du droit les composantes de l'environnement considérées comme « inutiles ». Plus encore, on peut craindre un enfermement de la pensée environnementale dans un cadre conceptuel réducteur, à partir duquel il devient impossible d'interroger et éventuellement de repenser notre rapport au monde vivant. Le concept de services écosystémiques, mobilisé pour sensibiliser les sociétés humaines à la nécessité de préserver les écosystèmes, pourrait les rendre aveugles à une approche plus fine et complexe de l'environnement ${ }^{28}$. Le risque est d'autant plus grand que si le droit peut rendre compte selon nous de la réalité nécessairement complexe des rapports entre humains et non humains ${ }^{29}$, les catégories et qualifications juridiques

27. Farjat G., 2002, Entre les personnes et les choses, les centres d'intérêts. Prolégomènes pour une recherche, RTDCiv., 221-236.

28. Norgaard R-B., 2010, Ecosystem services: From eye-opening metaphor to complexity blinder, Ecological Economics, 69, 1219-1227 : "What started as a humble metaphor to help us think about our relation to nature has become integral to how we are addressing the future of humanity and the course of biological evolution. The metaphor of nature as a stock that provides a flow of services is insufficient for the difficulties we are in or the task ahead. Indeed, combined with the mistaken presumption that we can analyze a global problem within a partial equilibrium economic framework and reach a new economy project-byproject without major institutional change, the simplicity of the stock-flow framework blinds us to the complexity of the human predicament. The ecosystem services approach can be a part of a larger solution, but its dominance in our characterization of our situation and the solution is blinding us to the ecological, economic, and political complexities of the challenges we actually face".

29. Coutellec L., Doussan I., 2012, Legal and ethical apprehensions regarding a relational object. The case of the genetically modified fish, Journal of Agricultural and Environmental Ethics, 25 (6), 861-875. 
trouvent néanmoins souvent leur ancrage dans une vision duale et utilitariste du monde.

En outre, le concept de service écosystémique se coule sans remous dans une idéologie néolibérale qui pense la nature en termes de « capital », de « flux », « d'agents naturels » et en propose une approche dématérialisée qui se prête à la financiarisation. Pour certains auteurs, les services écosystémiques « constituent l'archétype de la marchandise fictive, dont la seule finalité est d'être échangée, comme si le concept n'avait été forgé que pour cela $\aleph^{30}$. On remarquera à cet égard que le mécanisme de la compensation écologique participe du même courant, en particulier depuis les ajouts de la loi du 8 août 2016 qui institue des " unités de compensation » destinées à combler le « passif » créé par l'auteur d'un projet portant atteinte à l'environnement.

Mais le concept de services écosystémiques peut également être porteur d'une approche renouvelée du droit de l'environnement.

En effet, les services écosystémiques emportent aussi avec eux une approche systémique, dynamique de l'environnement. Si chronologiquement, l'intégration d'une approche systémique dans les textes juridiques est antérieure à l'apparition des termes même de services écosystémiques, ceux-ci pourraient constituer le moteur, ou le carburant, qui lui manquait ${ }^{31}$. Ainsi, les services écosystémiques peuvent contribuer à forcer, voire éclater, les frontières, les segmentations opérées par le droit. Par exemple, la protection d'espaces naturels tient compte aujourd'hui des fonctionnalités, des réseaux écologiques qui s'y nouent et ainsi de certains services écosystémiques rendus (épuration des eaux, régulation des parasites, services culturels par exemple), en matière de zones humides, de trames verte et bleue, ou encore du réseau Natura $2000^{32}$. L'évaluation des impacts d'une activité ou d'un projet d'aménagement est également susceptible d'être modifiée, et complexifiée, par la prise en compte des fonctions et services

30. Boisvert V., 2015, Les services écosystémiques : un nouveau concept ?. In : Le pouvoir de la biodiversité. Néolibéralisation de la nature dans les pays émergents, (Thomas F., Boisvert V., eds), collection Objectifs Suds, éditions Quae, 215-229, p. 227.

31. Fèvre M., 2016, Les services écosystémiques et le droit. Une approche juridique des systèmes complexes, thèse de doctorat en droit, Université Côte d'Azur, $712 \mathrm{p}$.

32. Cf. infra, M. Fèvre « Les "services écosystémiques", une notion fonctionnelle ». 
écosystémiques affectés. On peut également supposer que le concept de services écosystémiques amène de nouveaux acteurs, et l'on pense aux bénéficiaires de ces services, mais aussi aux « producteurs » ou « prestataires » de services environnementaux, et modifie le jeu des acteurs existants (pouvoirs publics, ONG, entreprises), emportant ainsi des effets en termes de " gouvernance » de l'environnement. Certains de ces mouvements de fond, créés, renforcés, accélérés par la diffusion des services écosystémiques dans le champ du droit sont déjà perceptibles ${ }^{33}$. Des analyses juridiques plus spécialisées, quand à la qualification de ces services, leurs effets en droit des contrats, et en particulier en matière de baux ruraux, en termes de responsabilité civile ou encore de dépense publique sont également à mener pour déterminer si les services écosystémiques peuvent être vus comme un nouvel objet juridique, comme le support de nouveaux rapports de droit, et s'ils sont de nature à modifier profondément les catégories et régimes juridiques existants. C'est tout l'intérêt des contributions qui suivent que d'explorer ces pistes et d'apporter des éléments de réponse.

33. Voir par exemple Fèvre M., 2016, op. cit ; A. Langlais (ed.), L'agriculture et les paiements pour services environnementaux : quels questionnements juridiques?, Presses universitaires de Rennes, à paraître, 2018. 


\title{
Les « services écosystémiques», une notion fonctionnelle
}

\author{
Mélodie FÈVRE \\ Docteure en droit \\ CREDECO/GREDEG, Université Côte d'Azur \\ IMBE, Aix-Marseille Université
}

\section{INTRODUCTION}

Au début des années 2000, les auteurs du rapport onusien du Millenium Ecosystem Assessment (MEA) postulent la dépendance de l'homme au bon état des écosystèmes, au travers des bénéfices qu'il en retire pour la satisfaction de son bien-être et de ses besoins élémentaires. La notion de "services écosystémiques 》 connaît alors une popularisation fulgurante. La classification internationale commune (CICES) décline ces services en une typologie, distinguant les services d'approvisionnement, les services de régulation et les services culturels.

D'emblée, la notion de « service écosystémique » présente une forte dimension pédagogique. D'une part, elle permet la collaboration et le dialogue entre les différentes disciplines autour de la plupart des thématiques environnementales, dont la lutte contre l'érosion de la biodiversité et des sols ${ }^{1}$. D'autre part, elle met en exergue les conséquences liées à la déconnexion des aspects de gouvernance socio-économique avec les contraintes écosystémiques, et alerte les décideurs sur les coûts que cela représente ${ }^{2}$. La notion irrigue alors assez rapidement les politiques publiques et les stratégies

1. Boisvert V., 2015, Les services écosystémiques : un nouveau concept ?, In : Le pouvoir de la biodiversité. Néolibéralisation de la nature dans les pays émergents, (Boisvert V., Thomas F.), IRD Editions, 1 ìre édition, coll. Objectifs Suds, QUAE, Marseille, p. 137.

2 Pushpam K. (dir.), 2012, The Economics of Ecosystems and Biodiversity : Ecological and Economic Foundations, Ed. Earthscan Ltd, Washington, p. 25. 
de protection de la nature, si bien que la question des services écosystémiques est presque systématiquement adossée à celle de la biodiversité.

Bien que construits à l'extérieur du droit, les services écosystémiques prennent aussi de l'ampleur en tant que notion juridique. Des régimes de réparation, de gestion, puis de prévention leur sont en effet associés $^{3}$. La définition juridique des services écosystémiques prête cependant le flanc à la critique. L'interprétation littérale de l'article L. 161-1-I-4 ${ }^{\circ}$ du Code de l'environnement ${ }^{4}$ conduit d'une part à une confusion, assez classique, entre les fonctions et les services. La référence à des fonctions bénéficiant à des " ressources naturelles » reste en effet fidèle à une approche éco-centrée, qui considère les fonctions des écosystèmes utiles à la biodiversité, et non uniquement utiles à l'homme. Or, une fonction ne prend la forme d'un service qu'à partir du moment où elle concourt à la satisfaction d'un besoin humain ${ }^{5}$. La rédaction de l'article limite d'autre part les services réparables aux services rendus par les eaux, les sols et les espèces et habitats protégés. La référence aux sols renvoie à un champ d'application très vaste et imprécis, qui complexifie la connaissance des fonctions ou services entrant dans cette catégorie. À l'inverse, la référence aux espèces et habitats protégés renvoie aux listes établies par les directives « Oiseaux » de 1979 (annexe I) et « Habitats » de 1992 (respectivement annexes II, IV et I), qui ne constituent qu'une représentation bornée de la diversité biologique.

Face à une définition juridique confuse, les sciences de l'écologie ne sont encore que d'un secours limité, tant il subsiste des difficultés à cerner avec exactitude les relations entre espèces, fonctionnement d'un écosystème et services écosystémiques ${ }^{6}$. Or, malgré ces limites, plusieurs auteurs présentent leur intégration comme une

3. Voir Doussan I., Brève histoire de l'intégration de la notion de service écosystémique en droit, supra.

4. Les « services écologiques » sont définis comme « les fonctions assurées par les sols, les eaux et les espèces et habitats... au bénéfice d'une de ces ressources naturelles ou au bénéfice du public, à l'exclusion des services rendus au public par des aménagements réalisés par l'exploitant ou le propriétaire ».

5. Boyd J., Banzhaf S., 2007, What are Ecosystem Services? The Need for Standardized Environmental Accounting Units, Ecological Economics, 63, p. 621.

6. Voir notamment Barnaud C., Antona M. et al., 2011, Vers une mise en débat des incertitudes associées à la notion de service écosystémique, VertigO-la revue électronique en sciences de l'environnement, [en ligne], 11 (1), http://vertigo.revues.org/, (consulté le 11 juillet 2017). 
innovation juridique, une avancée incontestable du droit, ou encore une consécration notable ${ }^{7}$. La notion de « services écosystémiques » pourrait ainsi ne pas être dénuée d'effets utiles. Si on reprend l'analyse du Doyen Vedel, les services écosystémiques pourraient être conçus comme une « notion fonctionnelle », à savoir une notion juridique caractérisée par une définition imprécise et instable, dont seule sa fonction lui confère son unité ${ }^{8}$.

On perçoit tout de suite le caractère fonctionnel des services écosystémiques du point de vue des politiques publiques, parce que la notion introduit une variable économique qui rend la préservation de la biodiversité « intéressante ». Cependant, quel peut être le rôle de la notion du point de vue du droit applicable à la protection et à la gestion de la nature ? En quoi contribue-t-elle à son évolution et à sa rénovation?

Les services écosystémiques ne dépendent pas directement des ressources naturelles, mais de l'intégrité et de la diversité des fonctions assurées par les systèmes écologiques ${ }^{9}$. À ce titre, des chercheurs définissent certaines fonctions écologiques par référence aux services dont elles constituent le fondement ${ }^{10}$. Appréhender un service écosystémique n'a donc de sens que si l'on considère

7. Desrousseaux M., 2014, La protection juridique de la qualité des sols, Thèse de doctorat, spécialité Droit, Université Jean-Moulin - Lyon III, p. 449 ; Fuchs O., 2008, Le régime de prévention et de réparation des atteintes environnementales issu de la loi du $1^{\text {er }}$ août 2008, A.J.D.A., 38, p. 2110, Hédary D., 2008, La loi du $1^{\text {er }}$ août 2008 sur la responsabilité environnementale : quel progrès pour l'environnement ?, Dr. Env., 163, p. 29.

8. Vedel G., 1950, La juridiction compétente pour prévenir, faire cesser ou réparer la voie de fait administrative, $J C P, 1950$ (I), 851, p. 425.

9. Wallis C., Blancher Ph., Séon-Massin N., Martini F., Schouppe M., 2011, Mise en cuvre de la directive-cadre sur l'eau. Quand les services écosystémiques entrent en $j e u, 2^{\text {ème }}$ séminaire "Quand les sciences de l'eau rencontrent les politiques publiques ", 29 et 30 sept. 2011, Bruxelles, Les rencontres de l'ONEMA, p. 27 ; Barbault R., 2011, La biodiversité, une façon écologique de comprendre notre monde, In : Quelle(s) valeur(s) pour la biodiversité ?, ECOREV, Revue critique d'écologie politique, 38, p. 11.

10. «The capacity of natural processes and components to provide goods and services that satisfy human needs, directly or indirectly », De Groot R.-S., Wilson M.-A., Boumans R., 2002, A typology for the classification, description and valuation of ecosystem functions, goods and services, Ecological Economics, 41, p. 394 ; « [...] the functions represent the potential that ecosystems have to deliver a service»; Pushpam K. (dir.), op. cit., p. 18 ; «les fonctions d'un écosystème sont les emboîtements nécessaires au niveau des écosystèmes, pour que la fourniture du service s'opère ", Aronson J., 2012, Regard d'un écologue sur la proposition de Nomenclature des préjudices environnementaux, In : Nomenclature des préjudices environnementaux, (Neyret L., Martin G.-J.), coll. Droit des Affaires, L.G.D.J. Lextenso, Paris, p. 54. 
la fonctionnalité qui le sous-entend ${ }^{11}$. Aussi, certains auteurs remarquent que " grâce à l'approche "services écologiques", la conservation de la biodiversité, à l'origine centrée sur la protection des espèces et des espaces souvent remarquables, prend en compte de manière croissante la fonctionnalité des écosystèmes $»^{12}$.

En 2008, Jean Untermaier voyait justement dans la considération des fonctions écologiques un nouvel objectif pour le droit applicable à la conservation de la nature ${ }^{13}$, tant leur érosion est directement liée à la déperdition en diversité biologique. Cette approche par les fonctions peine cependant à s'imposer en droit ${ }^{14}$. Les sols en sont un exemple illustratif. La communication de la Commission européenne de 2002 destinée à aller « Vers une stratégie thématique de la protection des sols », mais aussi la Charte européenne sur la protection et la gestion durable des sols, reconnaissent leur faculté à remplir une multitude de fonctions essentielles à la vie ${ }^{15}$. La proposition de directive européenne du 25 septembre 2006 sur la protection des sols ${ }^{16}$ est pourtant restée à $1^{1}$ état de projet ${ }^{17}$.

En outre, parce que les services écosystémiques reflètent l'étroitesse des liens qui unissent les sphères écologique et sociale, leur gestion,

11. Mission Économie De La Biodiversité, 2014, Les paiements pour Préservation des Services Ecosystémiques comme outil de conservation de la biodiversité. Cadres conceptuels et défis opérationnels pour l'action, CDC Biodiversité, Les Cahiers Biodiv'2050 : Comprendre, 1, p. 12.

12. Delangue J., 2015, Services écologiques : de quoi parle-t-on ?, Espaces Naturels, 52 , p. 25 ; Longeot J.-F., Dantec M., 2017, Rapport fait au nom de la commission d'enquête sur la réalité des mesures de compensation des atteintes à la biodiversité engagées sur des grands projets d'infrastructures, intégrant les mesures d'anticipation, les études préalables, les conditions de réalisation et leur suivi, Sénat, 517, p. 21.

13. Untermaier J., 2008, « Biodiversité et droit de la biodiversité », R.J.E., NS, p. 21.

14. Voir Décision n ${ }^{\circ} 1386 / 2013 / \mathrm{UE}$ du Parlement européen et du Conseil du 20 novembre 2013 relative à un programme d'action général de l'Union pour l'environnement à l'horizon 2020 « Bien vivre, dans les limites de notre planète ». Voir aussi, Muller-Curzydlo A., 2013, Vers une nouvelle directive sur la qualité des sols, Environnement, 8, alerte 162 et Billet Ph., 2015, Le sens des équilibres, In : Figures de la préservation de l'environnement outre-mer. Études de cas et réflexions pluridisciplinaires, (Naim-Gesbert É, Peyen L., Radiguet R.), PUAM, Aix-en-Provence, p. 183.

15. Charte européenne révisée sur la protection et la gestion durable des sols, adoptée par le Comité des Ministres le 28 mai 2003 lors de la 840 réunion des Délégués des Ministres, annexe 28, point 9.1 .

16. Commission Européenne, Proposition de directive du Parlement européen et du Conseil définissant un cadre pour la protection des sols et modifiant la directive 2004/35/CE, COM(2006) 232 final, Bruxelles, 25 septembre 2006.

17. Voir Hermon C., La protection du sol en droit, supra., et Doussan I., Brève histoire de l'intégration de la notion de service écosystémique en droit, supra. 
comme la prévention et la réparation de leurs atteintes, invite nécessairement à considérer la réciprocité des relations homme/ nature. Si les sciences de l'écologie globale ${ }^{18}$ ont pleinement conscience de l'importance de ces interactions, le droit les a souvent considérées comme rompues ou inexistantes, si bien que la protection de la nature s'est longtemps opérée en périphérie du développement humain. À mesure qu'elle se diffuse, la notion de « services écosystémiques » dicte cependant au législateur d'appréhender leur consubstantialité.

La reconnaissance des services écosystémiques a donc bien un rôle à jouer dans la lutte contre la dégradation des écosystèmes, en participant au renouvellement de la façon dont le droit appréhende les éléments naturels (I), et organise les rapports homme/nature (II).

\section{L'APPROCHE JURIDIQUE DE LA NATURE RENOUVELÉE}

L'unique définition juridique des « services écologiques » renvoie aux fonctions assurées par des éléments physiques et biologiques. En effet, la notion «traduit l'idée que dans la biodiversité [...] les interactions entre les espèces sont plus importantes que leur importance quantitative $»^{19}$. C'est ici déjà une évolution notable pour le droit de l'environnement, historiquement cantonné à la prise en compte isolée des éléments naturels. Cette approche par les fonctions n'est pas inconnue du droit, mais reste résiduelle, circonscrite à quelques législations sectorielles. La considération des services écologiques tend à renverser cette tendance, et à la rendre plus systématique (A). Prendre en compte les fonctions écologiques impose par ailleurs d'appréhender les conditions nécessaires à leur expression. Aussi, l'introduction des services écosystémiques dans le champ du droit démontre l'importance d'aborder le système écologique dans sa globalité (B).

18. Tatoni Th., Cramer W., Piégay H., Galop D., 2013, Pour une écologie globale, In : Prospective de l'Institut écologie et environnement du CNRS, (Thiébault S., Hadi H. (coord.)), compte-rendu des journées des 23 et 25 octobre 2012, Avignon, Les Cahiers prospectives, HS, pp. 219-224.

19. Martin G.-J., 2009, Les effets de la responsabilité environnementale : de la réparation primaire à la réparation compensatoire, Dr. env., 6, repère 6. 


\section{A. L'ENTRÉE DES FONCTIONS DE LA NATURE DANS LE CHAMP DU DROIT}

Avant que la notion de " services écosystémiques » n'y soit intégrée, le droit appréhendait déjà certaines fonctions du sol (1). À y regarder de près, la plupart de ces fonctions s'apparentent davantage à des services, signe que l'idée de fonctions socialement utiles avait déjà exercé une influence dans le champ juridique. Les récentes réformes montrent également comment leur prise en compte s'est peu à peu imposée dans divers régimes, signe que les services continuent d'exercer cette influence (2).

1) Les fonctions de la nature connues du droit avant l'apparition de la notion de « services écosystémiques $»^{20}$

Dès le début des années 2000 , le Code forestier reconnaît la multifonctionnalité des forêts, c'est-à-dire l'interdépendance de leurs fonctions économique, écologique et sociale. La fixation des sols dans les zones à forts risques naturels, l'accueil du public aux abords des villes, mais aussi la protection des équilibres biologiques constituent des utilités reconnues au couvert forestier, dont le législateur a organisé la sauvegarde. Étroitement liées à la fonction économique de la forêt, la reconnaissance des fonctions écologiques et sociales pose la question de leur gestion et du maintien de leur équilibre, compte tenu de leurs spécificités ${ }^{21}$. Le principe de « gestion durable » permet ainsi d'organiser la préservation des fonctions, en modulant la contrainte juridique au regard des enjeux locaux, des contextes géographiques et des priorités pour la collectivité. L'instrument « forêt de protection »" préserve quant à lui spécifiquement les couverts qui jouent un rôle contre les phénomènes de déclivité des sols en zone de montagne, mais aussi contre les phénomènes de mobilité des dunes sur les espaces littoraux soumis au risque d'affaissement ${ }^{23}$.

\footnotetext{
20. Ces développements, non exhaustifs, se limiteront à l'exemple des sols forestiers et des sols humides.

21. CGAAER, 2014, Trop exploitées, les forêts disparaissent, In : Controverse documentée à propos de quelques idées reçues sur l'agriculture, l'alimentation et la forêt. Ministère de l'agriculture, de l'agroalimentaire et de la forêt, mission $\mathrm{n}^{\circ} 13083$, ép. $\mathrm{n}^{\circ} 4, \mathrm{p} .12$.

22. Outils valant servitude d'utilité publique prévu à l'article L. 141-1, Nouveau C. forestier. 23. Art. L. 143-2 Nouveau C. forestier.
} 
C'est par ailleurs cette capacité naturelle des forêts à produire des fonctions et des services écosystémiques qui a justifié assez tôt que leur exploitation soit juridiquement encadrée. C'est également la reconnaissance tardive des fonctions et des services rendus par les zones humides qui conduit aujourd'hui le législateur à organiser leur sauvegarde, alors qu'il a longtemps encouragé leur assèchement.

La loi sur 1'eau du 3 janvier $1992^{24}$ intègre à 1'article L. 211-1I- $1^{\circ}$ du Code de l'environnement la protection des zones humides. La rédaction de l'article justifie cette nouvelle disposition par le concours de ces milieux à l'objectif général de gestion équilibrée de la ressource en eau. Ainsi, dès 1995, les zones humides sont qualifiées « d'infrastructures naturelles » par les politiques publiques, en raison « des nombreuses fonctions qu'elles assurent pour la collectivité $»^{25}$. Sans parler de « services », c'est donc encore une fois ce dont il est question $\mathrm{ici}^{26}$. Aussi, le législateur a vu dans les fonctions d'épuration et de régulation des zones humides ${ }^{27}$ un instrument de gestion qualitative et quantitative de la ressource en eau. En témoignent les orientations fondamentales des premiers schémas directeurs d'aménagement et de gestion des eaux (SDAGE), qui prévoyaient le maintien de ces fonctions, qui « jouent un rôle social à faire valoir auprès de la collectivité $»^{28}$. Il est à noter que si les lois et décrets ne parlent pas de « services », la plupart des SDAGE pris pour la période 2009-2015 ${ }^{29}$,

24. Loi n 92-3 du 3 janvier 1992.

25. Bazin P., Mermet L., 1999, L'évaluation des politiques « zones humides » de 1994 : son origine, son déroulement, ses résultats, Les annales des Mines, p. 89.

26. Pour certains auteurs, parler "d'infrastructures naturelles » conduisait en effet à reconnaître « l'importance de ces systèmes pour la société en raison des services qu'elles rendent », Fustec E., Lefeuvre J.-C., Barnaud G., 2000, De l'élimination à la reconquête des zones humides, In : Fonctions et valeurs des zones humides, (Fustec E., Lefeuvre J.-C.), Dunod, Paris, 2000, p. 5, Mermet L., 1995, Les infrastructures naturelles : statut, principe, concept ou slogan?, Zones humides Infos, 7, p. 9.

27. La protection des sols contre les inondations, la rétention et l'élimination des substances dissoutes en sont quelques exemples ; Barnaud G., Mermet L., 1998, Leçons à tirer de la procédure de délimitation des zones humides aux États-Unis, In : Zoner les espaces naturels? Objectifs, Méthodes et Perspectives, (Maurin H., Le Lay G., De Feraudy E.), Synthèse du séminaire tenu à Paris le 2 décembre 1996, MNHN, p. 62.

28. SDAGE RMC, 1996, Protection et gestion des milieux aquatiques et des zones humides, 20/12 (2), pp. 2 et 29.

29. Le SDAGE Seine-Normandie parle des « services environnementaux des zones humides » (SDAGE 2010-2015 du Bassin de la Seine et des cours côtiers normands, Orientations fondamentales, arrêté du 20 novembre 2009, p. 86), le SDAGE Rhin-Meuse parle de "services rendus » par les milieux aquatiques et les zones humides (SDAGE Rhin-Meuse, Orientations fondamentales, arrêté du 27 novembre 2009, pp. 27, 59 et 89), le SDAGE Rhône-Méditerranée parle aussi bien des " services rendus par les milieux 
puis 2016-2021 $1^{30}$ utilisent désormais la notion pour désigner, dans leurs objectifs et mesures, les fonctions des zones humides utiles à l'homme ${ }^{31}$. Ainsi, le SDAGE Rhône-Méditerranée souligne que « la préservation du bon fonctionnement des milieux est nécessaire à la biodiversité et utile à la société », relevant particulièrement les services essentiels des zones humides, dont leur rôle dans la prévention des risques naturels et dans la préservation de la dynamique fluviale ${ }^{32}$.

La loi DTR du 23 février $2005^{33}$ a par ailleurs réaffirmé l'importance des zones humides, en plaçant leur préservation et leur gestion au rang d'intérêt général ${ }^{34}$. Dès lors, le législateur a organisé la protection de leurs fonctions « utiles ». L'article L. 211-3-II $4^{\circ}$ a) du Code de l'environnement donne ainsi à l'autorité préfectorale la possibilité de délimiter de nouvelles zones soumises à contraintes environnementales (ZSCE) ${ }^{35}$, sur lesquelles seront établis des programmes d'actions visant à la protection d'intérêts collectifs supérieurs. Ainsi, des zones humides d'intérêt environnemental particulier (ZHIEP) correspondent à des zones dont le maintien ou la restauration présentent une forte capacité de régulation des débits des cours d'eau, une fonction d'auto-épuration, d'habitat d'espèces ou d'écrêtage des crues ${ }^{36}$. De surcroît, le schéma d'aménagement et de gestion des eaux (SAGE) peut définir des zones stratégiques pour la gestion de l'eau (ZSGE), sur lesquelles sont établies des servitudes d'utilité publique, dans l'hypothèse exclusive où ces zones humides jouent un rôle significatif dans la protection de la ressource en eau.

aquatiques » que des « services issus de la gestion des zones humides » (SDAGE RhôneMéditerranée, Vers le bon état des milieux aquatiques, Objectifs et programmes de mesures, arrêté du 20 novembre 2009, pp. 68 et 94), le SDAGE Adour-Garonne parle des « services rendus par les milieux aquatiques » (SDAGE Adour-Garonne, Orientations fondamentales, J.O. du $1^{\text {er }}$ décembre 2009, p. 11).

30. Des dispositions « visent à garantir les équilibres physiques et la capacité d'autoépuration des milieux aquatiques, soutenir les fonctions et services essentiels des zones humides ", SDAGE Rhône-Méditerranée, version présentée au comité de bassin du 20 novembre 2015, p. 51, SDAGE Adour-Garonne, janvier 2016, p. 82

31. Lucas M., 2014, La compensation écologique des zones humides en France : vers une intégration des services écosystémiques ?, Dr. Env., 219, p. 21.

32. SDAGE Rhône-Méditerranée, Orientation Fondamentale $n^{\circ} 1$, «Privilégier la prévention et les interventions à la source pour plus d'efficacité », Orientation Fondamentale n ${ }^{\circ} 2$, "Concrétiser la mise en œuvre du principe de non dégradation des milieux aquatiques », pp. 42,50 et 503 .

33. Loi n ${ }^{\circ} 2005-157$ du 23 février 2005.

34. Art. L. 211-1-1 C. env.

35. Dont le régime est prévu aux articles R.114-1 à R.114-10, C. rural.

36. Circulaire DGFAR/SDER/C2008-5030 du 30 mai 2008, op. cit., pp. 5 et 19. 
Les ZHIEP et ZSGE ne peuvent donc s'appliquer que sur les zones auxquelles la société reconnaît la fourniture de fonctions écologiques, sociales ou hydrologiques. Le législateur a ainsi créé des outils de protection non d'un milieu, mais des fonctions remplies par ce milieu. L'articulation entre ZHIEP et ZSGE témoigne par ailleurs de la façon dont le droit a hiérarchisé ces fonctions, en déployant un outil particulièrement contraignant pour la préservation des fonctions hydrologiques.

Une étude approfondie conduit au constat que dans les exemples choisis, c'est davantage des services (de régulation et culturels) que le droit préserve, sous la terminologie de $"$ fonctions $»^{37}$. Il faut dire que la réticence des parlementaires comme du gouvernement est encore palpable s'agissant de manipuler la notion de « services écosystémiques $»^{38}$. Quoiqu'il en soit, depuis son apparition, un accroissement de la protection juridique des fonctionnalités peut s'observer.

2) Les fonctions de la nature imposées au droit depuis l'apparition de la notion de « services écosystémiques »

À mesure que la notion de « services écosystémiques » se répand, on observe dans le champ du droit une prise en compte accrue des fonctions écologiques.

Cette considération nouvelle s'observe dans un premier temps dans la mise en œuvre des mesures de réparation du dommage ${ }^{39}$. La référence aux services écologiques dans la définition du dommage accidentel réparable par l'article L. 161-1-I-4 du Code de l'environnement contraint à ce que la remise en état soit étendue aux fonctions écologiques ${ }^{40}$. Cette approche vient corriger une pratique du droit désapprouvée bien plus tôt par la doctrine. En 1989, Martine Rémond-Gouilloud soulignait à propos de la réparation du préjudice

\footnotetext{
37. Fèvre M., 2016, Les services écologiques et le droit. Une approche juridique des systèmes complexes, Thèse de doctorat, spécialité Droit, Université Côte d'Azur, Nice, partie I.

38. Ibid., p. 370.

39. $C f$. infra $\mathrm{S}$. Jean «L'incidence des services écosystémiques en droit de la responsabilité civile».

40. Camproux-Duffrène M.-P., 2009, Les modalités de réparation du dommage ; apports de la « responsabilité environnementale, In : La responsabilité environnementale, prévention, imputation, réparation, (Cans Ch. (dir.)), Coll. Thèmes et commentaires, Dalloz, p. 116.
} 
causé au milieu que « [...] prendre en compte chaque spécimen pour lui-même, isolé de son contexte, conduit à oublier que sa valeur intrinsèque est indissociable de sa valeur écologique : ce qui compte ici, souvent, est non l'individu mais sa fonction, le rôle qu'il joue dans son écosystème $»^{41}$. C'est justement là ce qu'encourage la réparation des services écologiques, que soient restaurés autant les éléments détruits que les fonctions qu'ils remplissent ${ }^{42}$. Le législateur s'est d'ailleurs a priori donné les moyens de ses ambitions, en définissant " un régime de réparation multiple ${ }^{43}$. Ainsi, indépendamment des mesures de réparation primaire et complémentaire, la loi a introduit une mesure de réparation dite « compensatoire », qui vise à traiter systématiquement les pertes transitoires en fonctionnalités écologiques intervenues entre le moment du dommage et celui où les premières mesures ont commencé à produire leurs effets. Bien qu'elle ne reprenne pas ce triptyque de mesures, la loi pour la reconquête de la biodiversité, de la nature et des paysages $(\mathrm{RBNP})^{44}$ a également placé les fonctions au cœur de la définition du préjudice écologique ${ }^{45}$.

De la même façon, cette nouvelle exigence a été étendue par la loi RBNP aux fonctionnalités endommagées par un projet d'aménagement. L'article L. 110-1-II- ${ }^{\circ}$ du Code de l'environnement dispose ainsi que le principe de l'action préventive "implique d'éviter les atteintes à la biodiversité et aux services qu'elle fournit ; à défaut, d'en réduire la portée ; enfin, en dernier lieu, de compenser les atteintes qui n'ont pu être évitées ni réduites, en tenant compte des espèces, des habitats naturels et des fonctions écologiques affectées ». Selon le rapport de la commission d'enquête du Sénat, ce nouvel article considère désormais la biodiversité comme indissociable des services qu'elle fournit dans l'application du principe d'action préventive. Cette extension aux services

\footnotetext{
41. Rémond-Gouilloud M., 1989, Du droit de détruire - Essai sur le droit de l'environnement, P.U.F., Paris, pp. 224-225.

42. Martin G.-J., 2010, La responsabilité environnementale, In : L'efficacité du droit de l'environnement, (Boskovic O.), coll. Thèmes et Commentaires, Dalloz, Paris, p. 6.

43. Jarlie-Clément C., Gautier-Sicari M.-A., 2004, La directive sur la responsabilité environnementale, originalités et incohérences d'un régime juridique novateur, B.D.E.I., 4, p. 13.

44. Loi n ${ }^{\circ} 2016-1087$ du 8 août 2016.

45. Art. 1247 C. Civ., le préjudice écologique consiste en « une atteinte non négligeable aux éléments ou aux fonctions des écosystèmes ou aux bénéfices collectifs tirés par l'homme de l'environnement ».
} 
écologiques va nécessairement jouer sur la façon dont sera envisagée la réalisation des évaluations environnementales comme la mise en œuvre des mesures d'évitement, de réduction et de compensation, qui ne pourront plus ignorer la fonctionnalité des milieux ${ }^{46}$.

Enfin, de nouvelles fonctions ont récemment fait leur entrée dans les objectifs de protection du droit forestier. En France, le Code forestier reconnait depuis 1985 la fonction de protection des sols assurée par les forêts. En revanche, il faut attendre la loi d'avenir pour l'agriculture, l'alimentation et la forêt du 13 octobre $2014^{47}$ pour que les fonctions de protection de l'eau et de purification de l'air jouées par la forêt y pénètrent. Par ces nouvelles dispositions, le législateur reconnaît l'intérêt général qu'il y a à préserver les fonctions régulatrices jouées par les couverts forestiers ${ }^{48}$. Ici aussi, il est bien en réalité question de « services », comme l'avaient dès 2011 explicitement reconnu les parties à la Conférence ministérielle d'Oslo ${ }^{49}$.

À mesure que les connaissances scientifiques et les besoins sociaux évoluent, la popularisation de la notion de «services écosystémiques » conduit à ce que l'attention du législateur soit davantage portée sur les fonctions écologiques. La reconnaissance juridique des fonctions des écosystèmes forestiers et humides était donc bien déjà dictée par l'idée de préserver les fonctions utiles à la protection des hommes et des biens, c'est-à-dire des services.

Cependant, l'approche par les fonctions de la nature est longtemps restée circonscrite à ces seuls milieux. Plus percutante comme argument d'action que celle de «fonction », la notion de « services écosystémiques » a justement cela d'intéressant qu'elle permet de dépasser ces approches sectorisées. Faire de la protection des services écologiques un objectif du droit de l'environnement commande en effet que soient appréhendées de façon plus générale les fonctions écologiques qui les préfigurent, et partant, les conditions dans

\footnotetext{
46. Fèvre M., 2016, op. cit., pp. 454 et s.

47. Loi n ${ }^{\circ} 2014-1170$ du 13 octobre 2014

48. Art. L.112-1-4 ${ }^{\circ}$, C. forestier.

49. Les couverts forestiers « contribuent à atténuer les changements climatiques et à s'y adapter », et fournissent " une protection des eaux et des sols et d'autres services écosystémiques tout en protégeant la société et ses infrastructures des dangers naturels ", Conférence Ministérielle pour la Protection des Forêts en Europe, FOREST EUROPE, Oslo, Norvège, 14-16 juin 2011, considérant 7.
} 
lesquelles ces fonctions s'expriment, lors de l'élaboration et de l'application des règles relatives à la protection de la nature.

\section{B. L'ENTRÉE DU SYSTÈME ÉCOLOGIQUE DANS LE CHAMP DU DROIT}

Appréhender les fonctions écologiques à la base des services implique de s'intéresser non plus à une structure simple, mais à un système complexe, dont la prise en compte impose une approche méthodologique fondée sur la systémie, et non plus sur le statique et l'élémentaire. Ces exigences propres aux services écologiques conduisent inévitablement à renouveler la façon dont le droit appréhende les écosystèmes, dans une optique de protection comme d'exploitation. Alors que le législateur a tendance à simplifier les complexités écologiques, l'influence des services tend au contraire à les réincorporer, rappelant ainsi l'importance d'adopter une nouvelle approche de la nature (1). L'entrée récente de nouveaux objets dans le champ du droit en atteste (2).

\section{1) Une nouvelle approche de la nature}

D'un point de vue scientifique, les fonctions écologiques sont le résultat de processus et d'interrelations au sein d'un ensemble écologique complexe ${ }^{50}$. C'est donc lorsque l'on considère le système dans sa globalité que l'on appréhende les fonctions, interactions et processus intermédiaires à la production des services. C'est ici tout l'intérêt de l'approche systémique.

Le réseau Natura 2000 est l'exemple le plus illustratif de l'approche systémique de la nature par le droit. Les directives « Habitats » de 1992 et « Oiseaux » de 1979 sont en effet fondées sur la protection des espèces par celle des espaces ${ }^{51}$. Le réseau vise ainsi à protéger la fonction d'habitat d'espèces d'intérêt particulier, mais aussi les fonctionnalités plus génériques, via la protection des « habitats naturels », qui ne sont autres que des écosystèmes. Mais ce qui est particulièrement intéressant dans le dispositif Natura 2000, c'est que la préservation de ces fonctionnalités s'opère à l'échelle du réseau européen. Alors que parcelliser l'espace juridique revient à «bloquer toute possibilité d'une gestion globale et systémique du territoire et

50. Mission Économie De La Biodiversité, 2014, op. cit., p. 12.

51. Le Corre L., 2012, Réseau Natura 2000. Constitution. Régime de protection, JCL

Environnement et Développement durable, fasc. 3820, repère 4. 
des milieux qui s'y imbriquent $\aleph^{52}$, l'approche en réseau, propre au dispositif, s'attache à ce que chaque site soit désigné en considérant les exigences de cohérence écologique, de fonctionnalités et de connexité entre les écosystèmes. Et cette organisation en réseaux fonctionnels mène, de façon indirecte, à ce que les services écologiques soient protégés ${ }^{53}$. En 2010, la contribution de Natura 2000 à la régulation des aléas naturels, au développement des activités récréatives, à la purification de l'eau, et au maintien de l'agriculture et de la pêche était en effet considérable ${ }^{54}$. Cet état de fait conduisait les institutions européennes à reconnaître non seulement l'importance de la politique environnementale Natura 2000 dans la pérennité des services écosystémiques ${ }^{55}$, mais aussi et surtout l'intérêt de l'approche systémique dans leur fourniture ${ }^{56}$.

Il semble exister un lien presque indéfectible entre les modèles systémiques et le maintien des services écologiques ${ }^{57}$. Si cela paraît une évidence pour les sciences de l'écologie, tel n'est pas le cas en droit, où ce type de modèle et d'approche reste peu courant ${ }^{58}$. Pourtant, au-delà de la seule production de services, c'est toute la conservation de la nature qui pourrait être rendue plus efficace par

52. Le Louarn P., 1999, Les zones humides et le droit, CNFTP, SFDE, p. 35.

53. Brahic E., Terreaux J.-Ph., 2009, Évaluation économique de la biodiversité - Méthodes et exemples pour les forêts tempérées, QUAE, p. 23 ; Steichen P., 2004, Quels statuts juridiques pour les sites Natura 2000 ?, Études foncières, A.D.E.F, 18, p. 7.

54. Ten Brink P. Badura T., Bassi S., Daly E., Dickie H., Gantolier S., Gerdes H., Kettunen M., Lago M., Lang S., Markandya A., Nunes PALD, Pieterse M., Rayment M., Tinch R., 2011, Estimating the Overall Economic Value of the Benefits provided by the Natura 2000 Network, Final report to the European Commission, Institute For European Environmental Policy, Brussels.

55. Commission Européenne, 2011, Natura 2000 : les défis financiers du futur, L'Environnement pour les Européens, Magazine de la direction générale de l'environnement, 44, p. 3 ; Commission Européenne, Infrastructures vertes - Renforcer le capital naturel de l'Europe, $\operatorname{COM(2013)} 249$ final, 06/05/2013, Bruxelles, p. 9.

56. Décision n ${ }^{\circ}$ 1386/2013/UE du Parlement Européen et du Conseil du 20 novembre 2013 relative à un programme d'action général de l'Union pour l'environnement à l'horizon 2020 « Bien vivre, dans les limites de notre planète », point 22.

57. Maresca B., Mordret X., Ughetto A.-L., Blancher Ph., 2011, Évaluation des services rendus par les écosystèmes en France, Développement durable et territoires, [en ligne], 2(3), http:// developpementdurable.revues.org/9053, (consulté le 14 juin 2017); Ranganathan J. et al., 1998, Services d'écosystèmes. Guide à l'attention des décideurs, World Resources Institute (WRI), p. 2.

58. De Roany C., 2004, L'approche par écosystème pour la gestion des pêcheries - un concept en quête de définition, Dr. Env. 116, p. 45 ; Billet Ph., 2015, Le sens des équilibres, op. cit., p. 183. 
des politiques fondées sur une démarche systémique ${ }^{59}$, desquelles découlerait un traitement juridique uniforme et conforme aux unités écologiques ${ }^{60}$. L'introduction de la notion de "services écosystémiques » renouvelle donc l'importance de cette approche systémique, encore très sectorisée. Elle entraîne ainsi le droit de l'environnement dans une transition vers un nouveau modèle de protection, qui appelle à la prise en compte de nouveaux éléments.

2) De nouveaux objets de protection

La définition scientifique des services écologiques se fonde sur la notion d' " écosystème ", mais aussi sur celle de " processus $\rangle^{61}$ (a). À l'inverse de la définition juridique, elle n'opère pas de distinction sur la qualité des éléments naturels qui en sont à l'origine, de sorte à ce que la biodiversité dite « commune » tende à pénétrer les arcanes du droit (b).

\section{a) Les processus écologiques}

Les processus écologiques figurent à la base des fonctions écologiques. Plusieurs auteurs plaidaient pour leur reconnaissance juridique $^{62}$, et pour leur réglementation « dans leur complexité et leur irréductibilité " ${ }^{63}$. Qualifiée «d'urgente » en $1996^{64}$, la protection et la gestion des processus écologiques tardent à trouver un écho juridique en Europe ${ }^{65}$. Il est pourtant assez tôt acquis que la perte de certains processus est créatrice de risques pour la société humaine.

59. Dubois G., 2009, Écologie des coléoptères saproxyliques : Biologie des populations et conservation d'Osmoderma eremita (Coleoptera : Cetoniidae), Thèse de doctorat, Spécialité Biologie, Université de Rennes 1, p. 5.

60. Doumbé-Billé S., 1998, L'apport du droit international à la protection de la nature : la convention des Nations-Unies sur la conservation de la diversité biologique, In : 20 ans de protection de la nature, Colloque de la SFDE, 28-29 novembre 1996, P.U.L.I.M., Limoges, p. 198.

61. Selon la définition de Gretchen Daily, les services écologiques découlent « des conditions et des processus à travers lesquels les écosystèmes naturels, et les espèces qui en font partie, soutiennent et alimentent la vie humaine, Daily G., 1997, Nature's services: societal dependence on natural ecosystems, Island Press, Washington D.C., p. 3.

62. De Klemm C., 1998, Les législations de protection de la nature : les enseignements du droit comparé, In : 20 ans de protection de la nature, op. cit., pp. 232 et 237.

63. Naim-Gesbert É., 1997, Les dimensions scientifiques du droit de l'environnement, Bruylant et Vubpress, Bruxelles, p. 409.

64. Ibid., p. 234.

65. Ibid., p. 235 ; De Sadeleer N., 2009, La protection de la nature et de la biodiversité, In : Droit et politiques de l'environnement, Les Notices, La documentation française, Paris, p. 194. 
Cyrille de Klemm alertait en effet sur le fait qu' " une fois altérés, les processus deviennent incapables de fournir les services dont ont besoin la flore, la faune et les écosystèmes ${ }^{66}$ et conduisent souvent à « des désastres écologiques et des pertes économiques considérables $\aleph^{67}$. Ainsi en est-il de la plupart des processus du sol menacés par l'artificialisation, comme le recyclage, la décomposition, l'humidification, la modification des cycles et des nutriments, la détoxification ou encore la bio-remédiation.

Certains processus utiles à l'homme sont spécifiquement protégés par le droit. Les processus de lutte contre la déclivité des sols, mais aussi les processus sédimentaires qui concourent à l'épuration des eaux et au maintien des berges ${ }^{68}$ en constituent autant. D'autres sont en voie de reconnaissance, tels les processus de pollinisation sauvage ${ }^{69}$. À plusieurs égards, ces processus s'apparentent à ce que l'on peut appeler un service de régulation ${ }^{70}$.

La notion générique de " processus biologiques » a aujourd'hui les faveurs du législateur. Incorporés dans le titre relatif aux principes généraux du droit de l'environnement, les processus écologiques font désormais partie intégrante du patrimoine commun de la nation ${ }^{71}$. Il ressort des travaux législatifs relatifs au projet de loi RBNP que l'intégration de ce concept scientifique au Code de l'environnement, qualifiée " d'innovation », introduit « l'idée d'une biodiversité en perpétuelle interaction avec les différents éléments qui la composent (substrats, taxons, écosystèmes), et qu'elle doit s'appréhender de façon dynamique $»^{72}$. Plus spécifiquement, il faut y voir une nouvelle

66. De Klemm C., 1989, Les éléments de l'environnement, In : L'Écologie et la loi : le statut juridique de l'environnement : réflexions sur le droit de l'environnement, (Kiss A. (dir.), Carbiener R., Doumbé-Billé S., Fromageau J., Guttinger Ph.), coll. Environnement, L'Harmattan, Paris, p. 23.

67. Ibid.

68. Voir article L. 214-17 du Code de l'environnement sur la préservation des continuités écologiques des cours d'eau par les exploitants d'ouvrages hydrauliques.

69. Fèvre M., op. cit., p. 439.

70. À propos du droit forestier qui encadre strictement les fonctions écologiques cindyniques de la forêt, Éric Naim-Gesbert souligne que cette législation est bien protectrice « des processus écologiques », Naim-Gesbert É, 1997, op. cit, p. 378.

71. Art. L. 110-1-I al. 2 C. env.

72. Gaillard G., 2014, Rapport fait au nom de la commission du développement durable et de l'aménagement du territoire sur le projet de loi relatif à la biodiversité, Assemblée Nationale, 26 juin, p. 54. 
forme de prise en compte des services de régulation ${ }^{73}$. Tout est donc encore histoire de terminologie, mais nous retenons que c'est par le biais d'une lecture de la nature au prisme de ses services que les processus écologiques sont aujourd'hui intégrés plus franchement dans le champ du droit. La même remarque devrait pouvoir être formulée à l'égard des espèces dite « ordinaires ».

\section{b) La biodiversité ordinaire}

Le sol héberge une grande variété d'espèces ${ }^{74}$, dont la plupart sont encore peu connues. Pour invisibles qu'elles soient, ces espèces jouent un rôle indispensable au fonctionnement de l'écosystème global et sont à l'origine de divers processus écologiques, eux-mêmes à la base des fonctions et services. Micro-organismes (bactéries et champignons), macrofaune (fourmis, verres de terre), microfaune (nématodes), mésofaune (collemboles), macro-vertébrés (taupes), régulateurs biotiques, ou ingénieurs des litières en constituent autant ${ }^{75}$. À l'inverse de la biodiversité rare, menacée, ou qui présente un intérêt scientifique particulier, ces espèces communes ou ordinaires ne trouvent pas leur place dans les différents Codes. Il semble en revanche qu'elles puissent être valorisées au travers des services qu'elles rendent ${ }^{76}$. Sur le plan normatif, s'intéresser aux services de régulation pousse en effet plus que pour tout autre service à ne plus focaliser l'attention sur la nature remarquable ${ }^{77}$.

Partant de l'idée que l'article L. 110-1 du Code de l'environnement reconnaît d'intérêt général la sauvegarde des services écosystémiques, de nouveaux objectifs sont donc assignés à la conservation ${ }^{78}$. Cela va notamment conduire à étendre le champ de la protection en s'attachant à ces espèces communes, et partant, à revoir les droits qui s'y exercent. Cette reconnaissance de l'importance de la biodiversité

73. Delangue J., 2015, op. cit., p. 24.

74. Matthieu C., Lozet L., 2011, Dictionnaire encyclopédique de science du sol, Tec \& Doc, Lavoisier, p. 79.

75. Voir notamment Desrousseaux M., op. cit., p. 17.

76. Chevassus-Au-Louis B. et al., 2009, L'approche économique de la biodiversité et des services lies aux écosystèmes - Contribution à la décision publique, rapport, Centre d'Analyse Stratégique (CAS), p. 265 ; Mouysset L., 2015, Repenser le défi de la biodiversité. L'économie écologique, coll. Sciences durables, Rue d'Ulm, Paris, p. 55.

77. Couvet D., contribution orale à l'École thématique du CNRS, La notion de services écosystémiques et ses applications. Examen critique et interdisciplinaire, Montpellier, 10-14 juin 2013.

78. Untermaier J., 2008, op. cit., p. 31. 
ordinaire dans la production de services aura notamment un rôle à jouer dans l'appréciation par l'autorité administrative compétente de la légitimité des projets d'aménagements soumis à évaluation environnementale ${ }^{79}$.

Au-delà d'estomper la frontière entre les espèces et les espaces, le second mérite de l'approche par les services est de mettre en exergue le couplage entre écosystèmes et groupes sociaux ${ }^{80}$. La notion tend ainsi également à renouveler l'approche juridique du rapport de l'homme à la nature.

\section{L'APPROCHE JURIDIQUE DU RAPPORT HOMME/NATURE RENOUVELÉE}

Un service écosystémique se caractérise par la mise en relation d'une fonction et d'un avantage retiré par l'homme. La démarche systémique porte justement en elle cette capacité de dépasser les seules variables écologiques, pour absorber l'homme et sa place dans le système. À mesure que les services écosystémiques prennent de l'ampleur, une redéfinition de la relation homme/nature tend alors à se dessiner dans le champ du droit. Elle s'exprime notamment par l'apparition de nouveaux modèles productifs (A) et de nouveaux principes généraux (B).

\section{A. De nouveaux modèles PRoductifS}

La directive-cadre "Stratégie pour le milieu marin » de 2008 est le premier texte à fonder explicitement l'exploitation d'une ressource naturelle sur une approche écosystémique, dans le but de sauvegarder les services marins. L'approche écosystémique est une forme de démarche systémique, qui considère l'homme comme partie intégrante de l'écosystème. À ce titre, il l'influence et en retire des services. Il n'y a cependant pas que dans le cadre de l'exploitation des ressources marines que s'exprime le lien entre services rendus et approche systémique des milieux (1). Le droit

79. Voir « le raffermissement de la valeur de la nature via les services écologiques », Fèvre M., 2016, op. cit., p. 533 et s.

80. Mathevet R., Ritan C., Tatoni Th., 2015, Biodiversités et solidarités : au-delà des aires protégées, dessiner des « territoires capables, $h \& b$, La revue d'humanité et de biodiversité, 2, pp. 86-94. 
rural a aussi récemment intégré le concept d'agroécologie qui répond à ce même modèle (2).

1) L'approche systémique à l'œuvre dans l'exploitation des ressources marines

L'article L. 219-7 du Code de l'environnement transpose l'article 1.3 de la directive-cadre. Il dispose que la protection et la gestion du milieu marin visent à appliquer à la gestion des activités humaines une approche fondée sur les écosystèmes, qui garantisse "l'utilisation durable des biens et des services marins par les générations actuelles et à venir ». La dégradation des écosystèmes peut dès-lors se mesurer en termes de services perdus ${ }^{81}$. Aussi, l'article encourage la préservation des fonctionnalités écologiques dans le seul but d'assurer les services liés aux activités maritimes et littorales.

Cette approche par les écosystèmes se retrouve au cœur de la directive sur la planification de l'espace maritime du 23 juillet $2014^{82}$. Partant du constat que les écosystèmes marins en bonne santé rendent de multiples services, leur intégration dans les décisions de planification permet d'optimiser les avantages retirés par la société humaine ${ }^{83}$ en arbitrant les conflits entre les usages antagonistes. Appliquée à la planification, l'approche écosystémique permet ainsi de tenir compte des interactions entre les activités et usages existants et futurs, mais aussi des interactions synergiques entre ces activités et usages avec le milieu marin ${ }^{84}$.

Les services d'approvisionnement sont particulièrement concernés par la dégradation des écosystèmes. Faisant suite à l'entrée en vigueur de la nouvelle politique commune de la pêche le $1^{\text {er }}$ janvier 2014' $4^{85}$, la loi RNPB a introduit l'article L. 911-2-1 ${ }^{\circ}$ au Code rural et de la pêche maritime. En droit interne, les politiques de la pêche

81. Gambardella S., 2017, La(es) valeur(s) de la biodiversité marine à travers le prisme des quotas de pêche, In : Quelle(s) valeur(s) pour la biodiversité ? (Hautereau-Boutonnet M. Truilhé-Marengo E.), mare\&martin, p. 279.

82. Directive 2014/89/UE du Parlement Européen et du Conseil du 23 juillet 2014 établissant un cadre pour la planification de l'espace maritime.

83. Considérant 13, directive 2014/89/UE.

84. Article 8-2 et 6-2 a), directive 2014/89/UE.

85. Règlement (UE) n 1380/2013 du Parlement Européen et du Conseil du 11 décembre 2013 relatif à la politique commune de la pêche, article 4.17). 
maritime, de l'aquaculture marine et des activités halio-alimentaires sont dès lors encouragées à s'opérer « dans le cadre d'une approche écosystémique, afin de réduire au minimum les incidences négatives sur l'environnement $»^{86}$.

Il ressort de ces nouvelles dispositions que la façon la moins mauvaise de concilier les activités anthropiques avec la protection de l'environnement ne consisterait pas uniquement à maintenir et restaurer les éléments du milieu qui constituent la ressource, mais d'assurer également le bon fonctionnement de l'écosystème associé $^{87}$. Le souci de préserver les services d'approvisionnement en denrées marines et les services récréatifs encourage donc désormais les professionnels de la pêche et de l'aquaculture à maintenir en bon état les fonctionnalités écologiques et les interactions associés aux écosystèmes marins.

L'approche systémique se diffuse donc de façon très explicite dans la gestion générale du milieu marin. Elle a par ailleurs dépassé la seule question marine, pour gagner aujourd'hui le domaine agricole.

2) L'approche systémique à l'œuvre dans l'exploitation des ressources agricoles

La loi d'avenir pour l'agriculture, l'alimentation et la forêt du 13 octobre 2014 a introduit un livre préliminaire au Code rural et de la pêche maritime. Composé de deux articles, il est destiné à définir les finalités du droit rural. Dans un paragraphe II, l'article L. 1 énonce que « les politiques publiques visent à promouvoir et à pérenniser les systèmes de production agroécologique ». Il poursuit en précisant que ces systèmes sont " fondés sur les interactions biologiques et l'utilisation des services écosystémiques (...)». En effet, comme le souligne Carole Hermon, l'agroécologie « puise dans les services rendus par l'environnement et restitue des services à l'environnement $\|^{88}$. Parce que la notion de « services »y est centrale, l'agroécologie place le droit rural « dans une dynamique

86. Voir Curtil O., 2014, La réforme de la politique commune de la pêche, $R D$ rur., 424, dossier 15 , repère 3 .

87. Martin Ph., Projet de loi relatif à la biodiversité, Assemblée nationale, $\mathrm{n}^{\circ}$ 1847, 26 mars 2014, p. 37.

88. Hermon C., 2015, L'agroécologie en droit : état et perspective, R.J.E., 3, p. 420. 
systémique ${ }^{89}$, et les systèmes cultivés dans un espace beaucoup plus large, dans lequel devront être mis en évidence les échanges et les complémentarités ${ }^{90}$.

En exprimant la forte dépendance qui relie encore trop inconsciemment les sociétés humaines aux systèmes écologiques fonctionnels, la notion de « service » joue un rôle remarquable. Alors que l'agriculture conventionnelle donne l'illusion de pouvoir s'en affranchir par l'adjonction d'intrants, la notion de " service " rappelle à elle seule que les effets collatéraux des produits phytosanitaires (notamment le déclin des pollinisateurs et des populations d'espèces qui contrôlent les ravageurs), représentent un facteur réel de limitation des rendements. À l'inverse, l'utilisation des services de la nature via l'agroécologie permet la création d'un autre service, l'approvisionnement, et la préservation d'autres services, comme les services de régulation. Le modèle agroécologique va ainsi plus loin dans la prise en compte des complexités écologiques que ne le font les approches multifonctionnelles connues en droit forestier et en droit rural.

On peut aujourd'hui encore douter de la fonction et de l'opérationnalité des objectifs inscrits au titre préliminaire du Code rural et de la pêche maritime. Cependant, pour inciter davantage ce type de pratiques durables fondées sur l'utilisation et la production de services, de nouveaux principes ont émergé avec la loi RNBP.

\section{B. De NOUVEAUX PRINCIPES GÉNÉRAUX}

La loi RBNP a largement retravaillé l'article L. 110-1 du Code de l'environnement. Parmi les modifications apportées, il est intéressant de constater l'ajout de deux nouveaux principes, qui ont chacun

89. Trébulle F.-G., 2015, « Propos conclusifs », colloque sur « L'intégration du concept d'agroécologie en droit : état des lieux et perspectives, 22 mai 2014, Dr. Env., 230, p. 27, Hermon C., 2015, op. cit., p. 420. « L'agroécologie adopte un point de vue systémique, prend en compte les interdépendances et complémentarités des activités et des élément composants les écosystèmes ", Hermon C., 2016, Plaidoyer pour une simplification du droit relatif à la protection de l'environnement dans le secteur agricole, In : Les futurs $d u$ droit de l'environnement : simplification, modernisation, régression ? (Doussan I.), Colloque annuel de la SFDE, 20 et 21 novembre 2014, Bruylant, Bruxelles, p. 243.

90. «L'agro-écologie conduit en effet à prendre en compte la complexité des interrelations entre les plantes cultivées, leurs herbivores et les systèmes écologiques, voire les paysages dans lesquelles elles s'intègrent », Lepart J., Marty P., 2009, Sortir des espaces protégés pour conserver la biodiversité, Géographie et cultures, 69, p. 6, repère 21. 
vocation à réorganiser les rapports des hommes à l'environnement, mais aussi le rapport des hommes entre eux. Ainsi, le principe de complémentarité entre environnement et activités humaines reconnaît le rôle de l'exploitant dans la préservation des milieux et la fourniture de services écologiques (1). Quant au principe de solidarité écologique, déjà connu du droit, il impose de considérer les interactions entre les territoires dans les décisions publiques, et d'organiser la répartition des coûts et des avantages au-delà des limites administratives (2).

1) Le principe de complémentarité entre environnement et activités humaines

Le rôle de l'exploitant ou du propriétaire foncier dans le maintien des espaces naturels était déjà reconnu par quelques dispositions du droit rural et forestier. Il est aujourd'hui consacré par la loi RBNP, et intégré aux principes généraux du droit de l'environnement. Le $8^{\circ}$ de l'article 2 de la loi introduit en effet le principe de complémentarité entre l'environnement, l'agriculture, l'aquaculture et la gestion durable des forêts, visant notamment à garantir « des services environnementaux qui utilisent les fonctions écologiques d'un écosystème pour restaurer, maintenir ou créer de la biodiversité $»$. La question de la normativité de ce principe a longuement été débattue par les parlementaires ${ }^{91}$. Si l'on peut aisément douter de son caractère immédiatement prescriptif, le principe de complémentarité constitue un " guide interprétatif » dont devront se saisir les juges, la doctrine et les autorités en charge de la définition des politiques publiques $^{92}$. En effet, malgré une formulation qui laisse également planer la confusion sur les lignes directrices et le rôle de chacun ${ }^{93}$, il ressort nettement de ce nouvel alinéa la volonté de promouvoir les interactions socio-écosystémiques à la base des fonctions écologiques. En ce sens, le principe de complémentarité admet que le bon état des écosystèmes n'est pas exclusif de la présence humaine, mais plus encore, que certaines activités peuvent avoir un effet bénéfique sur leur fonctionnement, contribuant ainsi à la

91. Doussan I., 2017, Vive la complémentarité de l'agriculture et de l'environnement !, In : Loi Biodiversité. Ce qui change en pratique, (Cans Ch., Cizel O. (dir.)), Éditions législatives, pp. 77-81.

92. Voir Champeil-Desplats V., 2007, N'est pas normatif qui peut. L'exigence de normativité dans la jurisprudence du Conseil Constitutionnel, Cahiers du Conseil Constitutionnel, 21.

93. Doussan I., 2017, op. cit. 
préservation de la biodiversité et des services qui en découlent. En reconnaissant à ces exploitants la capacité de fournir des services environnementaux, le législateur leur assigne un rôle nouveau et ouvre ainsi la voie à ce que des mécanismes incitent ces acteurs à prendre part à la protection de la nature. Ici encore, la référence aux " services » semble intervenir pour favoriser l'acceptabilité sociale de la protection de la biodiversité et des fonctionnalités écologiques.

Cela étant, il y a souvent une dissociation entre les lieux qui produisent des services écosystémiques et les lieux qui en bénéficient, ce qui pose la question des solidarités écologiques ${ }^{94}$.

\section{2) Le principe de solidarité écologique}

En droit de l'environnement, la solidarité écologique intègre à la fois les relations écosystémiques et les relations homme/nature (a). Appliquée aux services écologiques, elle introduit assez naturellement la question du partage des coûts et avantages liés à leur protection et à leur gestion (b).

\section{a) De la solidarité des écosystèmes à la solidarité homme/nature}

La loi du 14 août $2006^{95}$ a introduit le principe de solidarité écologique dans le cadre très spécifique des parcs nationaux. L'article L. 331-1 du Code de l'environnement permet ainsi la prise en compte des phénomènes d'interface entre le cœur du parc et la zone d'adhésion, de sorte à permettre l'expression des flux, des processus et des fonctionnalités indispensables. L'article $2.3^{\circ}$ de la loi RBNP a quant à lui ajouté un sixième alinéa aux principes généraux de l'article L. 110-1 du Code de l'environnement. Désormais, la protection, la mise en valeur et la gestion des éléments constitutifs de l'environnement devront aussi s'inspirer du principe de solidarité écologique « qui appelle à prendre en compte, dans toute prise de décision publique ayant des incidences sur l'environnement, les interactions des écosystèmes, des êtres vivants et des milieux naturels ou aménagés ». La loi RBNP s'attache ainsi également à ce que soient considérés les phénomènes d'interface, à la différence

\footnotetext{
94. Chevassus-au-Louis B., 2011, Les services écologiques des forêts : définition des concepts, origines et typologies, Revue forestière française, LXIII (5), p. 222.

95. Loi ${ }^{\circ} 2006-436$ du 14 avril 2006 relative aux parcs nationaux, aux parcs naturels marins et aux parcs naturels régionaux.
} 
qu'elle y inclue l'homme via la référence à la notion globalisante d' " êtres vivants ». Ces interactions peuvent alors facilement se mesurer en termes de services rendus. Aussi, le législateur reconnaît que la perturbation d'un écosystème a des répercussions sur d'autres écosystèmes, et que la préservation des services écosystémiques implique de s'intéresser aux effets d'un aménagement ou d'une activité sur ces interrelations, notamment en termes de coûts et d'avantages.

b) Le partage des avantages et des coûts liés à l'entretien des services écologiques à l'échelle du territoire

Les derniers SDAGE encourageaient la considération des solidarités entre l'amont et l'aval des bassins-versants, au regard des services de régulation rendus par le premier au second, comme la purification de $1^{\prime}$ eau ${ }^{96}$. Durant les débats parlementaires concernant la loi RBNP, la députée Geneviève Gaillard présentait un amendement visant à proposer un principe de "solidarité écologique entre les territoires ", qui s'appliquerait aux seules autorités publiques. Il s'agissait là de considérer la solidarité dans sa dimension territoriale, selon laquelle « un écosystème, un milieu écologique complexe aux fonctionnalités évidentes, s'il coûte à une collectivité du point de vue de sa conservation et de sa valorisation, peut le plus souvent bénéficier à un territoire voisin ou non ${ }^{97}$. Ainsi, toute décision publique peut avoir un impact au-delà des limites de la collectivité, comme ce qu'il en est pour les bassins versants. À ce titre, ladite décision doit être en mesure de prendre en compte les interactions territoriales. Le principe de solidarité implique ainsi le partage des charges financières liées à l'entretien des écosystèmes en bon état de fonctionnement, avec les territoires qui en bénéficient ${ }^{98}$. Le bénéfice retiré du fonctionnement des écosystèmes correspond à la définition des services écosystémiques. La prise en compte des services écosystémiques par le droit amène donc bien à la promotion de vieux principes sectorisés, érigés en nouveaux principes directeurs. Les sciences de l'écologie auront d'ailleurs ici leur rôle à jouer, pour ce qui est de « documenter les dépendances entre socio-écosystèmes

96. Lucas M., 2014, op. cit., p. 24.

97. Gaillard G., 2015, Assemblée nationale, XIVe législature, Session ordinaire de 20142015, Compte rendu intégral, première séance du lundi 16 mars 2015.

98. Voir Icher L., La dépense publique en matière environnementale : l'exemple de la protection des sols et des services, infra, à propos de la loi montagne du 28 décembre 2016. 
fournisseurs de services écosystémiques et socio-écosystèmes bénéficiaires $\rangle^{99}$.

La visibilité de la notion de "services » ouvre donc aujourd'hui la voie à une nouvelle approche territorialisée des problématiques environnementales et à une gestion partagée, soucieuse des équilibres globaux. Les services écosystémiques constituent peut-être une première clé pour aller vers un droit qui s'extrait des catégories juridiques et des limites territoriales, pour appréhender au plus juste les dynamiques écologiques.

\section{CONCLUSION}

En définitive, les services écologiques dépassent les fonctions dans le potentiel qu'ils recèlent pour le droit. La richesse et la fonctionnalité de la notion de « service écologique » se situent donc dans son influence sur les transformations en cours, qui touchent aux fondements même du droit. Le propos n'est pas ici d'attribuer aux services écologiques le mérite exclusif de ces évolutions, entamées avant son apparition par les notions de « biodiversité » et de « développement durable ». En revanche, la juridicisation de la notion accélère et conforte ces changements nécessaires, entraînant avec elle l'entrée de nouveaux modèles, de nouveaux outils et de nouvelles notions, à la faveur d'une approche qui reconnaît et considère la complexité des systèmes socio-écologiques. Considérant son rôle dans l'élaboration de normes plus en phase avec les exigences écologiques, la notion de " services écosystémiques » est bien, selon nous, une notion fonctionnelle. Si nous en sommes convaincus, il faudra cependant prendre garde à ce qu'elle n'en vienne pas à occulter la notion de « fonction », ni à ouvrir la voie à la domination d'un droit des espèces et des espaces « utiles $»^{100}$.

99. Bosi S., Euzen A., 2015, Prospective droit, écologie et économie de la biodiversité, Les Cahiers Prospectives, CNRS, p. 12.

100. Doussan I., Brève histoire de l'intégration de la notion de service écosystémique en droit, supra. 


\title{
La qualification juridique des services écosystémiques
}

\author{
Guillaume BEAUSSONIE \\ Professeur \\ Université Toulouse-Capitole \\ IEJUC, F-31000
}

Dans le cadre de l'Évaluation Française des Écosystèmes et des Services Écosystémiques (programme national EFESE) les « biens et services écosystémiques » sont définis comme « des avantages socioéconomiques retirés par l'homme de son utilisation durable des fonctions écologiques des écosystèmes $»^{1}$. Pour polémique qu'elles soient ${ }^{2}$, cette appellation de « biens et services écosystémiques » et la définition ainsi proposée renvoient à un véritable concept, autrement dit désignent une réalité au moins doublement perceptible : d'abord, il est question d'appréhender un écosystème comme étant porteur d'utilité(s) pour l'homme, ce qui se conçoit aisément ; ensuite, presque à l'inverse, il s'agit d'appréhender l'homme comme étant capable de valoriser ou, pour le moins, de conserver un écosystème, ce qui se comprend tout aussi facilement.

Dans ce contexte, la place du Droit apparaît naturelle : le bénéfice, la protection et la valorisation d'un objet lato sensu, quel qu'il soit, se réalisent rarement de pur fait. Quand bien même cela serait le cas, ce qui n'est pas totalement inconcevable, il faudrait au moins prévoir, voire prévenir, tout conflit éventuel quant à la possession - au sens premier - de cet objet, à plus forte raison parce qu'il procède en partie de la nature, que l'on présente usuellement comme n'appartenant à personne. Il faut donc du Droit. À cette fin, le Droit doit faire entrer

\footnotetext{
1. V. glossaire de cet ouvrage.

2. Doussan I., Brève histoire de l'intégration de la notion de service écosystémique en droit, supra.
} 
les services écosystémiques dans sa propre réalité, c'est-à-dire qu'il doit procéder à leur qualification.

Le Droit est, dans sa construction, prêt à recevoir les faits. Il les désigne abstraitement, à travers des concepts, l'association de différents concepts permettant de mettre en œuvre la règle de droit. Seul le rattachement d'un fait à un concept juridique va alors permettre d'appliquer une règle à partir de ce fait. Plus précisément, le fait va entrer dans une catégorie juridique préexistante afin de se voir appliquer le régime - autrement dit les règles - correspondant. Ce rattachement est la qualification juridique.

Les choses sont simples quand une étiquette adéquate existe, c'està-dire lorsque le Droit crée lui-même ou consacre expressément le concept qu'il dote d'un régime juridique complet. Tel n'a pas été le cas, pendant longtemps, des services écosystémiques, dont la qualification ne pouvait donc qu'être qu'empruntée à d'autres concepts, déjà appréhendés par le Droit. Aujourd'hui, une qualification existe, notamment - mais pas seulement - dans le Code de l'environnement mais, outre qu'elle manque de clarté, elle ne conduit qu'à une partie du régime auquel peuvent prétendre les services écosystémiques.

Ainsipeut-on lire, dès l'article L. 110-1 duditCode, que le «patrimoine commun de la nation [...] génère des services écosystémiques et des valeurs d'usage », la sauvegarde de ces services étant "d'intérêt général », " concour[ant] à l'objectif de développement durable » et constituant même l'un des cinq engagements figurant à la fin ce texte inaugural. Dans ce but, l'un des principes affirmé par le législateur est celui "d'éviter les atteintes à la biodiversité et aux services qu'elle fournit ; à défaut, d'en réduire la portée ; enfin, en dernier lieu, de compenser les atteintes qui n'ont pu être évitées ni réduites, en tenant compte des espèces, des habitats naturels et des fonctions écologiques affectées $»^{3}$. C'est d'ailleurs à ce seul sujet que, en la matière, le Code de l'environnement dépasse justement la pétition de principe, l'affectation des services "écologiques» - dont il semble qu'ils ne soient, bien maladroitement, qu'un autre

3. Ces affirmations sont récentes ; elles sont effectivement issues de la loi $\mathrm{n}^{\circ} 2016-1087 \mathrm{du}$ 8 août 2016 pour la reconquête de la biodiversité, de la nature et des paysages. 
nom donné au services "écosystémiques $»^{4}$ - étant perçue comme un dommage causé à l'environnement qui, en tant que tel, peut et doit être prévenu et réparé en vertu des règles figurant aux articles L. 160-1 et suivants. À cette occasion, les services écologiques sont même définis comme «les fonctions assurées par les sols, les eaux et [les espèce et habitats protégés] au bénéfice d'une de ces ressources naturelles ou au bénéfice du public, à l'exclusion des services rendus au public par des aménagements réalisés par l'exploitant ou le propriétaire », c'est-à-dire des services « environnementaux». Dans le plus récent article 1247 du Code civil, à propos de la réparation du préjudice écologique, on définit ce préjudice, de façon encore plus contemporaine, comme " une atteinte non négligeable aux éléments ou aux fonctions des écosystèmes ou aux bénéfices collectifs tirés par l'homme de l'environnement $»^{5}$.

À la fin, malgré leur plus large vocation, qui ressort tant de leur définition positive que de l'ambition qu'affiche, à leur égard l'article L. 110-1, les services écosystémiques - ou écologiques - ne sont donc mobilisés que négativement, comme les supports éventuels d'un dommage réparable. Ce n'est pas étonnant, tant le droit de l'environnement s'est construit avant tout comme un droit protecteur, plus que comme un droit valorisateur'. Mais c'est insuffisant, en ce sens que ce qui est protégé l'est, la plupart du temps, en raison de sa valeur, valeur qui transparaît par une utilité dont la privation conduit, effectivement, à une réparation. L'essentiel semble donc là, dans cette valeur des services écosystémiques qui les rend utiles en tant que tels et qui, lorsque l'on y porte atteinte, conduit à la rétablir au profit de ceux qui en sont les bénéficiaires. Il est alors nécessaire de rattacher ces objets à la qualification juridique qui exprime le mieux cette essence.

\footnotetext{
4. Dans le même sens, v. Fevre M., Les services écologiques et le droit, Une approche juridique des systèmes complexes, thèse, Nice, 2016, p. 68. - Ce vocabulaire n'est pas encore stabilisé, sans doute pour la raison qui précède : la jeunesse de l'appréhension juridique desdits services.

5. V. dans cet ouvrage, Jean S. «L'incidence des services écosystémiques en droit de la responsabilité civile $»$.

6. V. par ex. Hermon C., « Plaidoyer pour une simplification du droit relatif à la protection de l'environnement dans le secteur agricole ", in Les futurs du droit de l'environnement. Simplification, modernisation, régression (dir. Doussan I.), Bruylant, 2016, p. 235.
} 
En Droit, on distingue fondamentalement les sujets, c'est-à-dire les personnes, qui bénéficient des règles juridiques, des objets, à savoir les choses, qui sont le support de l'application desdites règles.

Les services écosystémiques sont des choses au sens large ou, si l'on préfère rester prudent, sont rattachables à des choses : sols, eaux, espèces etc. Les choses, en effet, représentent tous les objets, matériels ou immatériels, qu'une personne est susceptible de posséder : voiture, château, mais aussi étang, vache ou encore information. En cela ces choses constituent-elles, parce que, de bien des façons, elles sont utiles aux personnes, l'enjeu et l'objet de l'ensemble des relations juridiques entre ces dernières. Elles les échangent et garantissent, par leur entremise, tous leurs engagements.

Que les services écosystémiques soient ainsi les utilités d'une chose ou la chosification de ces utilités, les questions principales en ce qui les concerne demeurent celles du bénéfice de ces utilités et de l'atteinte susceptible de leur être portée. Autrement dit se pose d'abord, en droit, la question de la jouissance des services écosystémiques, c'est-à-dire de la détermination de celui ou ceux qui peut ou peuvent prétendre bénéficier de leurs utilités ou de la compensation de celles qui ont été perdues.

Parallèlement, les services écosystémiques sont des choses ou renvoient à des choses que, en raison de leur lien avec l'environnement, on n'utilise pas n'importe comment. C'est dire que, en droit, se pose ensuite la question de l'encadrement de la jouissance des services écosystémiques, bref de la réglementation de leur usage.

À ce stade, où il s'agit donc essentiellement de déterminer à qui sont rattachables les services écosystémiques et ce qu'il peut en faire, c'est-à-dire de savoir qui va pouvoir en jouir et en disposer dans la limite des lois et des règlements, c'est bien sûr de propriété dont il est question. Les services écosystémiques sont donc, en première analyse, des objets de propriété, c'est-à-dire ce qu'on appelle des biens, ou des éléments d'un tel objet, c'est-à-dire des utilités.

À l'issue de ce qui précède, il ne faudrait pourtant pas croire que le problème de la qualification juridique des services écosystémiques soit totalement réglé. Il existe, en effet, bien des objets de propriété - « un œuf ou un bœuf, mais aussi les usines Renault, un billet de $100 \mathrm{~F}$, 
l'étang de Ville d'Avray » s'amusait à souligner Carbonnier ${ }^{7}$-, dont la nature juridique particulière conduit, au-delà du régime commun à tous, à un régime propre à chacun. Ce qui signifie, d'abord, que rien ne s'oppose à ce que les services écosystémiques soient effectivement perçus comme des biens ou, du moins, comme les éléments d'un ou de plusieurs biens ${ }^{8}$. Ces services ne feraient alors que s'inscrire au sein de ces nouveaux biens que l'évolution des sociétés humaines a conduit à consacrer ${ }^{9}$ : informations, droits de natures diverses etc.

Toutefois, cette situation implique, ensuite, que ces objets inédits que représentent les services écosystémiques suscitent, au sein même du droit de la propriété, des questions nouvelles, que seule une qualification juridique plus précise - et le régime qui va avec apparaît susceptible de résoudre. Faut-il, en effet, faire évoluer le droit des biens au regard d'une inadaptation manifeste de ses règles actuelles - créer une nouvelle qualification - ou, au contraire, ce droit peut-il déjà, en l'état, accueillir ces biens issus de la modernité - en mobilisant une qualification préexistante?

La réponse peut être trouvée, dans un premier temps, dans la détermination, au regard de ce que constitue la propriété en droit et, plus précisément, de son objet, de la particularité des services écosystémiques : ils ont des traits propres qui les singularisent au sein des objets de propriété (I). Sur cette base, il est possible, dans un second temps, de confronter l'objet ainsi déterminé avec des qualifications déjà existantes : les services écosystémiques ont, en effet, des traits communs avec d'autres objets de propriété (II).

\section{LES TRAITS PROPRES AUX SERVICES ÉCOSYSTÉMIQUES AU SEIN DES OBJETS DE PROPRIÉTÉ}

Les services écosystémiques sont utiles à l'homme ; c'est une certitude, car telle est la cause de leur consécration et de leur promotion

7. Carbonnier J., Les biens, Quadrige, PUF, 2004, $19^{e}$ éd., n 707.

8. La littérature est déjà très importante concernant une telle hypothèse, qui semble la principale envisagée. V. par ex. Martin G.-J., «Les biens-environnements, une approche par les catégories juridiques », RIDE, 2015/2, p. 139.

9. Revet T., "Les nouveaux biens ", Rapport français, in Travaux de l'Association H. Capitant, t. 53, La propriété, 2003, n 19, p. 285 : «En fonction des données culturelles, économiques et sociales qui la déterminent, chaque grande période de l'histoire des sociétés humaines s'articule, notamment, sur un modèle de bien ». 
juridiques. En cela le Droit ne fait-il que rejoindre ce que l'écologie ou encore l'économie ont déjà révélé ${ }^{10}$.

Passé ce constat, néanmoins, les choses se compliquent car, d'une part, ces services expriment davantage - voire sont - l'utilité des écosystèmes pour l'homme que, à proprement parler, ils ne sont utiles en eux-mêmes. Autrement dit, leur utilité est totalement intrinsèque (A). D'autre part, les services écosystémiques sont, plus exactement, utiles aux hommes, à l'humanité en général ou, pour le moins, à un groupe plutôt qu'à un homme en particulier. En ce sens, leur utilité est commune (B).

\section{A. Une UTILITÉ INTRINSÈQUe}

«Les biens sont les choses dont l'utilité justifie l'appropriation $»^{11}$. L'utilité est, ainsi, consubstantielle à la notion de bien, c'est-à-dire d'objet de propriété. Et elle l'est doublement, car seules les choses utiles, mais aussi dont la réservation apparaît utile, sont appropriées. C'est l'une des raisons de la communauté de certaines choses, dont l'abondance n'oblige pas encore à l'appropriation, malgré leur utilité intrinsèque indéniable : air, lumière naturelle, eau courante etc. Toutefois, dans un monde où tout devient possible en raison des avancées scientifiques, il peut surtout être utile d'éviter que ces choses appartiennent à quelqu'un. Il $\mathrm{y} a$, autrement dit, les choses qu'on ne peut pas s'approprier et celles qu'on ne veut pas qu'elles soient appropriées. Toutes sont des choses communes.

Quoi qu'il en soit, pour qu'une chose devienne un bien, c'està-dire soit appropriée, ce qui importe est son utilité pour une ou plusieurs personnes. C'est dire que cette utilité n'est jamais prise en compte qu'en considération de propriétaires potentiels, bref de façon extrinsèque et subjective. Cela explique la difficulté à délimiter la notion de bien.

La seule certitude est que, dans le système moderne, la chose utile et, pour cette raison, appropriée, appartient totalement à son propriétaire. Devenue bien, la chose conserve son, ou plutôt ses utilités intrinsèques, qui vont alors, de façon monopolistique,

10. V. dans cet ouvrage, Doussan I., « Brève histoire de l'intégration de la notion de service écosystémique en droit ».

11. Zenati-Castaing F., Revet Th., Les biens, PUF, 2008, $3^{\mathrm{e}}$ éd., n 2. 
profiter à une personne unique : le propriétaire. C'est l'apport de la modernité, en effet, que d'avoir fait du bien le seul élément de rattachement de l'ensemble des utilités de la chose qu'il qualifie. Cela conduit, bien souvent, à assimiler la raison qui a conduit une personne à s'approprier une chose, l'utilité extrinsèque, et les qualités de la chose appropriée, ses utilités intrinsèques, qui demeurent avant comme après l'appropriation mais bénéficient au propriétaire exclusivement, au-delà même de ce qu'il souhaitait faire de la chose ${ }^{12}$.

L'évolution des techniques a permis, dans certaines situations, d'autonomiser l'une des utilités intrinsèques d'un bien. Par exemple, la chambre criminelle de la Cour de cassation a, dans un arrêt notoire rendu le 14 novembre $2000^{13}$, considéré que le numéro de carte de crédit pouvait constituer un bien au même titre que la carte ellemême. Issue de cette dernière, le numéro de carte de crédit n'en comporte pas moins une utilité propre, la comparaison entre les deux objets ainsi dessinés apparaissant, à cet égard, très éclairante.

La carte et son numéro ont effectivement une utilité similaire, mais pas commune : permettre, par leur remise respective, la circulation des fonds du remettant. De telle sorte que l'on peut parfaitement concevoir, comme l'a fait la chambre criminelle de la Cour de cassation en l'espèce, que l'on puisse respectivement et indépendamment les remettre et les détourner ${ }^{14}$. L'utilité du numéro de carte de crédit a donc fini par en faire une chose autonome puis, en raison de son utilité, un bien.

De ce point de vue, il est possible d'envisager que les services écosystémiques, au moins singularisés par la loi par rapport à l'écosystème dont ils sont issus, soient des utilités que l'on peut traiter juridiquement de façon autonome. Mais il reste, en vérité, deux grandes difficultés, notamment en comparaison du numéro de carte de crédit.

12. V., à cet égard, notre étude : «Bien(s) et utilité(s)», in Mélanges Grégoire Forest, Dalloz, 2014, p. 39.

13. Bull. crim., $\mathrm{n}^{\circ} 338$.

14. V. Cass. crim., 19 mai 2004, Bull. crim., $\mathrm{n}^{\circ} 125$ : sanction, cette fois, du détournement de la carte de crédit elle-même. Évidemment remis avec la carte, le numéro n'a pas été utilisé par l'auteur du détournement. Classiquement, il n'a en effet usé (puis abusé) que de la carte et de son Code d'accès (qui se distingue de son numéro !). 
La première difficulté est l'absence de dissociation matérielle entre les services écosystémiques et les écosystèmes. En effet, où le numéro de carte de crédit peut parfaitement exister indépendamment de la carte elle-même, tel ne semble pas être le cas des services écosystémiques, qui ne s'autonomisent qu'intellectuellement des écosystèmes dont ils sont issus ${ }^{15}$. Toutefois, une telle objection peut être discutée, en ce sens que les services assurés paraissent bien perceptibles indépendamment des biens qui les génèrent. C'est d'ailleurs la cause de leur prise en compte, notamment par le Droit. Le véritable problème réside alors surtout dans la fugacité de leur existence, donc de leur perception.

La seconde difficulté est, en effet, le caractère éphémère des services écosystémiques. Où le numéro de carte de crédit survit à son utilisation, les services écosystémiques sont ontologiquement consomptibles. Ils n'existent que le temps d'être rendus, ce qui ne facilite pas leur appréhension, juridique ou autre. En cela, ils sont comparables davantage avec des services au sens du Droit, dont le régime s'avère adapté à leur évanescence, qu'à celui des biens au sens strict, qui supposent une inscription pérenne dans la durée ${ }^{16}$.

Toutefois, certains services écosystémiques paraissent en vérité, malgré leur évanescence, tout aussi durables que les biens qui les génèrent. Par exemple, le service de captation du carbone par une prairie ne disparait qu'avec la prairie.

Le numéro de carte de crédit est un bien ; qu'en est-il des services écosystémiques? À l'aune de ce qui précède, il est encore difficile de le dire, l'autonomie et la stabilité qui caractérisent une telle notion demeurant encore polémiques pour de tels services. Ce d'autant que ces services présentent une autre particularité au regard du droit des biens : ils ont ou sont des utilités communes.

\section{B. UNE UTILITÉ COMMUNE}

Le regroupement des utilités au sein d'un même bien, qui caractérise la propriété moderne, a un corollaire, qui en serait même plutôt

\footnotetext{
15. V. intervention de Mme Vanuxem S., La nature des services écosystémiques en droit privé, IDEX, T2SEC, IEJUC, Toulouse, 26 mai 2016.

16. V. intervention de M. Revet Th., La nature des services écosystémiques en droit privé, IDEX T2SEC, IEJUC, Toulouse, 26 mai 2016.
} 
la cause : c'est l'exclusivité de leur bénéfice pour le propriétaire. Dès lors, chacune des utilités d'un bien, qu'elles soient actuelles ou virtuelles, n'a vocation à profiter qu'à un seul : le propriétaire. En ce sens, la notion de bien commun, pourtant si séduisante, apparaît antinomique, du moins aux yeux du juriste classique, seules des « choses » communes, c'est-à-dire inappropriables ou, du moins, inappropriées, étant concevables. Les définir et les doter d'un régime sont d'ailleurs les objets précis de l'article 714 du Code civil ${ }^{17}$.

Or, les services écosystémiques ont des bénéficiaires, si ce n'est universels, en tout cas multiples. Ils profitent, en effet, à des hommes plutôt qu'à un homme en particulier. En ce sens, outre que les propriétaires des supports d'écosystème dont procèdent les services écosystémiques ne s'avèrent pas toujours en mesure de s'opposer à leur utilisation par d'autres qu'eux, il est même concevable, puisque telle est la condition de l'utilisation de ces services, que leur soient imposées des obligations positives ${ }^{18}$.

Il existe différentes situations, néanmoins, dans lesquelles un propriétaire doit déjà accepter que les autres bénéficient d'une partie des utilités de son bien, soit que lui ou un précédent propriétaire s'y soit engagé, soit que la loi lui impose.

La plus notoire, sans doute, est celle du droit de reproduire un bien, que la Cour de cassation a, dans la logique de l'article $544 \mathrm{du}$ Code civil, dans un premier temps refusé de retirer du monopole du propriétaire avant que, dans un second temps, face à l'impossibilité et l'inopportunité d'empêcher la photographie des immeubles dans la rue, elle l'oblige à la partager, sauf à ce qu'il démontre que cette utilisation lui cause un trouble anormal ${ }^{19}$.

La comparaison avec les services écosystémiques apparaît pertinente, en ce que l'image du bien semble tout aussi indissociable de ce dernier que ne le sont les services du bien qui supporte l'écosystème.

17. « Il est des choses qui n'appartiennent à personne et dont l'usage est commun à tous (al. 1). Des lois de police règlent la manière d'en jouir (al. 2) ». - De moins en moins de juristes sont gênés par cette idée : v. par ex. Dictionnaire des biens communs, dir. Cornu M., Orsi F. et Rochfeld J., Quadrige-PUF, 2017.

18. Intervention de M. Revet Th., La nature des services écosystémiques en droit privé, préc.

19. V. Cass. civ. $1^{\text {ère }}, 10$ mai 1999, Bull. civ. I, n 87, puis Cass. ass. plén., 7 mai 2004, Bull. A. P., $\mathrm{n}^{\circ} 10$. 
Mais elle a aussi des limites : l'image survit à sa reproduction, où les services succombent parfois dès leur consommation. Cela étant, beaucoup d'entre eux semblent, en réalité, tout aussi consubstantiels au bien qui les génère que peut l'être l'image d'un bien (ex. : service de régulation des inondations; service de captation du carbone).

Quoi qu'il en soit de cette différence, rien ne parait s'opposer à ce que les utilités d'un bien soient partagées entre le propriétaire et les autres, à la condition, néanmoins, que toutes les utilités - voire la majorité d'entre elles - ne lui soient pas retirées, faute de quoi il ne serait plus propriétaire d'un objet qui, par là même, ne serait plus un bien. Ce serait une chose commune. À cet égard, l'utilité commune ne doit pas conduire à évincer totalement les utilités privées, à défaut de quoi serait alors en cause une privation de propriété ${ }^{20}$.

Les services écosystémiques n'empêchent pas les propriétaires des biens supportant les écosystèmes qui les génèrent de les utiliser. Tout au plus ne doivent-ils pas limiter cette utilisation de façon trop substantielle, car il serait alors porté atteinte au droit des propriétaires. Ne faudrait-il pas, dès lors, prévoir une compensation de ce débit d'utilité, ce que ne prévoient pas les textes pour le moment ? Cela est d'autant plus concevable que, sur ce point comme sur d'autres, les services écosystémiques ressemblent finalement à d'autres objets de propriété auxquels il paraît conséquemment concevable de les assimiler.

\section{LES TRAITS COMMUNS AUX SERVICES ÉCOSYSTÉMIQUES ET À D'AUTRES OBJETS DE PROPRIÉTÉ}

En tant qu'entités juridiques autonomes, la loi les singularisant des biens dont ils sont issus, les services écosystémiques sont des choses lato sensu; des choses provenant d'autres choses en somme. Comme on l'a dit, « chose » est un mot qui, en droit français, désigne tout ce qui existe - même sans consistance physique - à l'exception des personnes juridiques et tout ce qui, en tant que tel, a donc vocation à devenir l'objet d'un droit dont ces dernières seront titulaires. Encore faut-il, néanmoins, que l'autonomie des services écosystémiques

20. Contraire, notamment à la Constitution : v. par ex. déc. nº 81-132 DC du 16 janv. 1982 et toutes les décisions qui ont suivi. 
soit véritable ; or nous avons pu constater que leur détachement des biens dont ils proviennent demeurait polémique. En conséquence, deux pistes distinctes doivent être suivies : d'une part, percevoir un service écosystémique comme l'utilité d'une chose (A); d'autre part, appréhender un tel service comme une utilité faite chose (B).

\section{A. Une UTILITÉ D'UNE CHOSE}

La propriété moderne étant, comme on l'a dit, une et indivisible, la piste du service qui ne constituerait pas un bien mais, simplement, l'une de ses utilités, est la plus sécurisante. Deux éléments doivent alors être combinés pour déterminer ce que sont juridiquement les services écosystémiques : le lien envers la chose dont ils sont issus ; leur utilité commune.

D'une telle combinaison s'infèrent plusieurs qualifications possibles : fruits communs, servitudes administratives, mesures de police environnementale ou obligations réelles.

Tout d'abord, Madame Vanuxem, partant du constat que les services écosystémiques sont, en tant que bienfaits de la nature, des choses générées régulièrement par d'autres choses sans altération de la substance de ces dernières, proposent de les qualifier de fruits naturels, au sens donné à cette notion par interprétation classique de l'article 583 du Code civil, qui les définit comme « ceux qui sont le produit spontané de la terre $»^{21}$. C'est à la condition, précise-telle, d'abord, que les services écosystémiques soient perçus comme détachés des biens dont ils sont issus, ce qui est concevable, au moins intellectuellement. Et c'est à la condition, ensuite, que ces fruits nouveaux ne soient pas appréhendés comme des biens, mais comme une nouvelle forme de choses communes.

Cette proposition, néanmoins, malgré sa vertu de mettre en valeur le lien entre les services écosystémiques et les biens qui supportent les écosystèmes dont ils sont issus, peut conduire à une double objection. D'abord, la notion de fruit comporte-t-elle vraiment un intérêt ainsi déliée de son enjeu majeur, qui est de désigner le propriétaire de ces biens accessoires à d'autres biens? Il s'agit, en effet, du possesseur

21. Intervention de Mme Vanuxem S., La nature des services écosystémiques en droit privé, préc. 
du bien générateur du fruit. Ensuite, peut-on vraiment concevoir que s'épanouisse une chose commune au sein d'une chose qui, quant à elle, est appropriée, c'est-à-dire propre ${ }^{22}$ ? Propre et commun s'opposant, cela paraît difficile à justifier d'un point de vue logique.

L'une des caractéristiques des services écosystémiques pouvant être d'imposer, pour l'utilité commune, des obligations aux propriétaires des biens qui les génèrent, la figure de la " servitude » administrative apparaît ensuite la plus adaptée ${ }^{23}$. Charge réelle, c'est-à-dire obligation que supporte un fonds de subir une restriction d'utilité destinée à mettre cette utilité au service d'un autre que le propriétaire, la servitude administrative semble correspondre à la mécanique qui est celle d'un service écosystémique. La servitude administrative se fonde effectivement sur l'utilité générale, de sorte que son bénéficiaire est, inéluctablement, un public indéterminé. En somme il existe un fonds servant, mais pas vraiment de fonds dominant.

Les services écosystémiques pouvant être supportés par un fonds pour l'intérêt général, ils limitent par là même, comme on l'a vu, les prérogatives de son propriétaire. En cela peut-on pertinemment $\mathrm{y}$ voir des formes de servitudes administratives. Il existe déjà, au surplus, de nombreuses servitudes fondées sur les préoccupations pour l'environnement, la lutte contre la pollution et les risques technologiques, la protection du patrimoine architectural et urbain ou encore la mise en valeur des paysages.

À la condition que les services écosystémiques ne soient pas perçus comme des charges réelles, on peut encore voir dans les obligations de protection qu'ils portent des mesures de police environnementale. Toutefois, en l'occurrence, si obligations il y a, elles sont bien dues, si ne n'est par le fonds, en tout cas à travers le fonds. Dès lors, il s'agit, sans aucun doute, de charges, la piste des mesures de police devant donc être écartée - aucune demande d'autorisation pour user des services n'est encore imposée au propriétaire -, du moins pour

22. Objection mise en avant par M. Revet Th. : La nature des services écosystémiques en droit privé, préc.

23. Cette proposition et les suivantes sont celles de M. Revet Th. : La nature des services écosystémiques en droit privé, préc. Il ne s'agit pas, en l'occurrence, d'une servitude au sens du droit civil, le service dû « par le fonds » ne l'étant alors pas au profit d'autres fonds, comme cette notion l'implique pourtant (v. art. 637 C. civ.). 
le moment, car elle ne serait pas la moins efficace pour prévenir les risques d'atteinte à ces services.

Les services écosystémiques peuvent, enfin, être perçus comme des obligations réelles, c'est-à-dire des obligations qui, parce qu'elles sont liées à une chose, sans laquelle elles ne peuvent s'exercer, se transmettent de propriétaire en propriétaire. Cette approche apparaît d'autant plus pertinente qu'a récemment été consacrée l' " obligation réelle environnementale ${ }^{24}$. En effet, l'article L. 132-3 du Code de l'environnement dispose désormais que « les propriétaires de biens immobiliers peuvent conclure un contrat avec une collectivité publique, un établissement public ou une personne morale de droit privé agissant pour la protection de l'environnement en vue de faire naître à leur charge, ainsi qu'à la charge des propriétaires ultérieurs du bien, les obligations réelles que bon leur semble, dès lors que de telles obligations ont pour finalité le maintien, la conservation, la gestion ou la restauration d'éléments de la biodiversité ou de fonctions écologiques ».

Les services écosystémiques peuvent ainsi être appréhendés comme des obligations, pour les propriétaires successifs de biens supportant des écosystèmes, de laisser tout ou partie des autres bénéficier de leurs bienfaits. Ces obligations sont réelles en ce qu'elles ont effectivement pour base les services rendus par un bien. Toutefois, on voit immédiatement la limite d'une telle analyse : contrairement à l'obligation réelle environnementale, pour laquelle le bénéficiaire est peut-être universel, mais le débiteur se limite, sans aucun doute, au cocontractant désigné - collectivité publique etc. -, le service écosystémique a, parfois, un débiteur tout aussi universel - tout le monde ne doit-il pas laisser les écosystèmes produire leur bienfait ? - que ne l'est son bénéficiaire. Dès lors, il apparaît difficile de concevoir un véritable rapport d'obligation car, comme une obligation, au sens strict et juridique du terme, ne peut être due à tout le monde - la fameuse obligation passive universelle de Planiol -, elle ne peut pas non plus être due par tout le monde. Tout cela est bien sûr à relativiser selon les services concernés.

À partir du moment où le propriétaire d'un bien supportant un écosystème doit subir l'utilisation des services rendus par ce dernier

24. Cf. infra Alidor B. « Compensation et services écosystémiques ». 
au profit de tous, ne faudrait-il pas compenser, par un paiement, ce déficit d'utilité pour lui, autrement dit instaurer des " paiements pour services écosystémiques » ? Pour intéressante qu'elle soit, cette possibilité, pour le moment, plus prospective que positive - encore que l'on peut déjà identifier certaines obligations positives pour les propriétaires de biens offrant de tels services ${ }^{25}$-, concerne davantage les situations dans lesquelles une intervention humaine s'avère nécessaire, autrement dit les services environnementaux plutôt que les services écosystémiques. Tant que ces derniers services pourront être rendus sans impliquer activement le propriétaire, le paiement ne se justifie pas suffisamment par la diffusion d'utilités qu'il ne serait de toute façon pas en mesure d'empêcher. Il en ira peut-être autrement lorsque seront exigées de la part des propriétaires de biens supportant des écosystèmes de véritables obligations positives.

Lorsque la source d'un bienfait est un bien, l'obligation d'en faire bénéficier tout le monde représente une charge pour le propriétaire. C'est sous cet angle qu'il apparaît possible de qualifier les services écosystémiques, sortes de servitudes environnementales imposées aux propriétaires de biens supportant des écosystèmes.

Toutefois, il existe un autre angle à explorer afin de qualifier les services écosystémiques : c'est, à l'inverse, celui de ses bénéficiaires. De leur point de vue, l'utilité ainsi conférée ne devient-elle pas un bien ou, pour le moins, une chose?

\section{B. UNE UTILITÉ FAITE CHOSE}

On l'a dit : il arrive, malgré le caractère indivisible de la propriété moderne, qu'une utilité se détache d'un bien et devienne, en tant qu'entité autonome, un nouveau bien. Au surplus, il est alors concevable que cette utilité soit attribuée à un autre que le propriétaire du bien qui l'a générée. C'est le cas, surtout, de certains biens reconnus par la jurisprudence de la Cour européenne des droits de $l^{\prime}$ 'homme construite sur le fondement de l'article $1^{\text {er }}$ du Protocole $n^{\circ} 1$, en vertu duquel toute personne « a droit au respect de ses biens », et, plus particulièrement, par l'arrêt Öneryildiz contre Turquie, rendu le 30 novembre $2004^{26}$.

25. V. dans cet ouvrage, Poumarède M., « Services écosystémiques et contrat. Quelle obligation contractuelle environnementale?».

26. Req. $n^{\circ} 48939 / 99$. 
Cette décision, en effet, a conduit à protéger, sur ce fondement, une personne qui occupait illégalement un taudis, bien dont elle ne pouvait donc pas, en principe, être reconnue comme étant propriétaire. La Cour européenne n'en a pas moins considéré que " les autorités [avaient] de facto reconnu que l'intéressé et ses proches avaient un intérêt patrimonial tenant à leur habitation », cet intérêt étant « suffisamment important et reconnu pour constituer un intérêt substantiel, donc un «bien» au sens de la norme exprimée dans la première phrase de l'article $1 \mathrm{du}$ Protocole $\mathrm{n}^{\circ} 1 »^{27}$. Dès lors, bien que la chose concernée ait, sur les fondements traditionnels du droit des biens, une appropriabilité contestable, son utilité pour le requérant, quant à elle, ne souffrait pas polémique. Plus encore, c'était même, au-delà de l'utilité, dans le besoin que se situait en réalité l'intérêt de la chose ainsi déterminée, besoin dont l'évidence a conduit à la consécration d'une propriété très éthérée.

Plus prosaïquement, parce qu'une personne avait intérêt à ce que son habitation soit perçue comme sa propriété, quand bien même elle n'était fondée sur rien d'autre que cet intérêt, bref cette utilité, la Cour européenne l'a consacrée en tant que telle.

Les services écosystémiques ne sont-ils pas également, au-delà de leur utilité, des besoins pour l'humanité ? En ce sens, ne sont-ils pas les biens de tous?

La conception qui précède heurte la logique du droit français, qui n'autorise un bienfait commun qu'à la condition, inverse, que la chose concernée ne soit pas appropriée. C'est même précisément pour cela qu'elle ne l'est pas. On reconnaît l'article 714 du Code civil, qui dispose qu' '« il est des choses qui n'appartiennent à personne et dont l'usage est commun à tous ». Les services écosystémiques seraient ainsi, à condition qu'ils soient détachés de leur source, dès le moment où ils sont détachés de leur source, des choses communes.

Toutefois, comme on 1'a dit plus haut, il faudrait alors admettre que le propre, le bien contenant les écosystèmes, puisse produire du commun, les services écosystémiques, autrement dit qu'une désappropriation s'opère à partir du moment où les services écosystémiques se détachent de leur base. La comparaison faite par 
Madame Vanuxem avec les fruits ${ }^{28}$ redevient alors envisageable, les fruits devenant la propriété du possesseur plutôt que du propriétaire.

Mais une telle perception a ses limites : outre que, pour les fruits, il ne s'agit que de désigner un autre propriétaire, et non d'en dénier l'existence, comme pour les choses communes, cet autre propriétaire l'est car il a un lien avec la chose principale ; il la possède, ce qui veut dire qu'il en jouit et, de la sorte, va pouvoir affecter les fruits à son entretien. Rien de tel, pour les services écosystémiques, qui échappent purement et simplement à leur propriétaire et à leur bien d'origine.

Est-il utile, dès lors, d'en faire des choses autonomes ; ne vaut-il pas mieux se contenter de les percevoir comme de simples utilités communes d'un bien qui demeure propre?

En faisant preuve de prudence, et face à la nécessité de rester conforme à la notion de propriété, qui est la technique que le législateur français semble avoir mobilisée pour appréhender les services écosystémiques, à l'instar de tout objet avec lequel il s'agit d'instaurer une relation juridique de sujet à objet, il vaut mieux percevoir ces services comme les parties d'un bien que comme des biens propres. En ce sens, ils en représentent une utilité, dont la particularité est de bénéficier à plusieurs ou à tous, bien au-delà de leur seul propriétaire, qui ne saurait, la plupart du temps, en empêcher le déploiement. Leur régime, c'est-à-dire leur traitement juridique actuel, parait effectivement tributaire de cette nature.

28. Intervention de Mme Vanuxem S., La nature des services écosystémiques en droit privé, préc. 


\section{LES SERVICES ÉCOSYSTÉMIQUES RENDUS PAR LES SOLS DU POINT DE VUE DE L'AGRONOMIE}





\title{
Agriculture de conservation des sols et services écosys- témiques
}

\author{
Ariane CHABERT \\ Docteure en agronomie \\ post-doctorante Idex T2SEC \\ Jean-Pierre SARTHOU \\ Professeur, INRA/INP-ENSAT
}

Les agroécosystèmes sont des espaces d'interface entre l'Homme, ses pratiques et son savoir-faire, et les ressources naturelles. Contrairement aux écosystèmes naturels, les agroécosystèmes ont une orientation productive marquée et l'Homme influe sciemment sur leurs paramètres biologiques et physico-chimiques dans le but d'assurer quantitativement et qualitativement la production agricole via notamment l'irrigation, la fertilisation et le contrôle des bioagresseurs (adventices, maladies et ravageurs). Ce n'en sont pas moins des écosystèmes, ils sont le siège de flux de matières et d'interactions biologiques mais également sources d'habitats et de services. L'agriculture moderne, fondée sur un paradigme industriel, présente des impacts désormais avérés sur le capital naturel (Pretty et al., 2000), notamment sur les sols ${ }^{1}$. Ces systèmes agricoles montrent aujourd'hui leurs limites, notamment du fait de la rétroaction négative de leur impact sur l'environnement (pollution et raréfaction des ressources naturelles, émission de gaz à effet de serre, bioagresseurs résistants aux substances phytosanitaires...). Ils sont moins résilients et leur durabilité est remise en cause, en particulier dans un contexte de changements globaux.

1. V. supra Chabert A. et Sarthou J.-P., « Le sol agricole, une ressource indispensable négligée ». 
Pour être durable, l'agriculture de demain se doit de diminuer sa dépendance aux intrants non renouvelables, intégrer les processus biologiques et écologiques, impliquer les acteurs locaux et favoriser des actions collectives (Pretty, 2008). Ces préoccupations agroécologiques poussent aujourd'hui des producteurs, de plus en plus nombreux, à réinventer leurs systèmes pour les rendre à la fois rentables, résilients et cohérents avec leur environnement. Cette approche écosystémique de l'agriculture a ainsi donné naissance à une diversité d'agricultures alternatives aux systèmes dits conventionnels, comme l'agriculture biologique, l'agroforesterie, la permaculture, l'agriculture de conservation des sols mais également l'intégration de pratiques comme les couverts végétaux, les associations de culture, la protection intégrée, la lutte biologique par conservation, etc. Ces agricultures sont aujourd'hui institutionnalisées ou reconnues à des niveaux très divers et assurent toutes ensemble la transition agroécologique qu'il est nécessaire d'entamer.

Dans ce travail de réflexion autour de la protection des sols, nous avons choisi d'étudier spécifiquement les services écosystémiques en agriculture de conservation des sols. Non pas que nous présumons de la plus grande efficience agroécologique de cette forme d'agriculture sur d'autres pratiques dont certaines, comme l'agriculture biologique, sont par ailleurs bien connues pour la biodiversité qu'elles supportent. Mais le paradigme de l'agriculture de conservation est centré sur la question des sols. Il s'agit donc pour nous de mesurer les services rendus par cette agriculture.

La conservation des sols est un axe particulièrement intéressant pour la transition vers un engagement agroécologique. Relégué par l'agriculture industrielle au rang de support ${ }^{2}$ " inerte », dont il est même possible de s'affranchir (cultures hors-sol), le sol est un écosystème à part entière, hébergeant des processus écologiques de grande importance (voir encart) (Lemanceau et al., 2015).

Le travail du sol pour l'ameublir, préparer le lit de semence, gérer les adventices, améliorer la fertilité, etc., est une pratique ancestrale qui remonte à quelque 3000 ans avant J-C (Hobbs et al., 2018) et représente la base de nombreux systèmes agricoles.

2. Parallèle intéressant, le sol est également relégué au rang de « support » en droit civil. V. supra Bosc L., « Réflexions civilistes sur la prise en compte de la qualité des sols ». 
Les processus écologiques, fondement du concept de qualité du sol.

- Productivité primaire : capacité d'un sol, naturel ou cultivé, à produire de la biomasse végétale à des fins utilitaires pour l'Homme (alimentation humaine et animale, fibres, combustibles) ;

- Purification et régulation de l'eau : capacité d'un sol à extraire des composés toxiques présents dans l'eau qu'il renferme, et à recevoir, stocker et restituer l'eau pour une utilisation ultérieure par l'Homme, selon des modalités qui préviennent les événements de sécheresse et inondation prolongées et d'érosion ;

- Séquestration et régulation du carbone : capacité d'un sol à réduire les impacts négatifs sur le climat d'une augmentation des émissions de gaz à effet de serre dans 1 'atmosphère (e.g. $\mathrm{CO}_{2}, \mathrm{CH}_{4}$ et $\mathrm{N}_{2} \mathrm{O}$ );

- Fourniture de biodiversité fonctionnelle et intrinsèque : la multitude des organismes et processus écologiques du sol en interaction avec toutes ses composantes, constituant une part fonctionnellement importante du capital naturel du sol et fournis sant à la société un large éventail de services culturels et de services en core inconnus;

- Fourniture et recyclage des nutriments : capacité d'un sol à recevoir des nutriments sous la forme de sous-produits, à fournir des nutriments à partir de ressources intrinsèques, à acquérir des nutriments des compartiments air et eau, et à transférer ces nutriments dans des denrées végétales récoltées.

Ces processus écologiques sont par conséquent à l'origine de divers services écosystémi ques, s' exprimant simultanément mais à des niveaux inégaux et variables sel on les types de systèmes de production (Rutgers et al., 2012; Schulte et al., 2014). 
Cependant, cette perturbation systématique de l'écosystème sol fut remise en question à la fin des années 30, aux États-Unis, suite aux gros problèmes d'érosion et des événements climatiques catastrophiques rencontrés lors de cette décennie («Dust Bowl»). La pratique du labour est pour la première fois pointée du doigt pour les risques d'érosion qu'elle engendre (Faulkner, 1943). C'est alors qu'une agriculture alternative, l'agriculture de conservation des sols (ACS) se développe aux États-Unis pour lutter contre l'érosion des sols liée à la sécheresse et au vent, mais également pour faire face à la pression économique grandissante liée à l'augmentation des prix du pétrole (Friedrich et al., 2012).

Cette nouvelle forme d'agriculture dont le paradigme est le maintien de la cohésion naturelle des agrégats du sol, repose sur trois axes, rassemblant un ensemble de pratiques, tous trois destinés à assurer de concert une protection et un renforcement tant physique que biologique de la santé des sols (Farooq and Siddique, 2015a; FAO, 2008) : la réduction maximale du travail du sol (jusqu'à son annulation), la diversification des espèces cultivées dans la rotation culturale et la couverture permanente du sol par des couverts ou débris végétaux (Fig. 1).

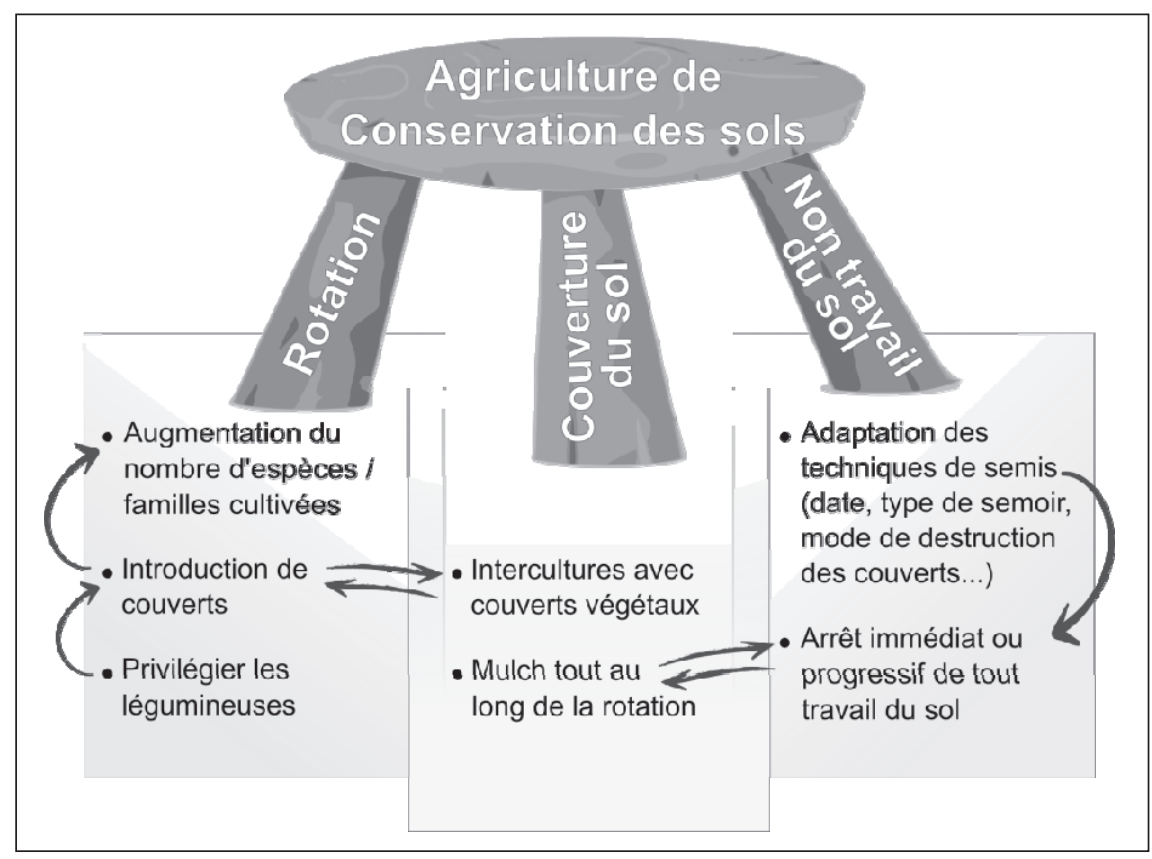

Figure 1. Particularités des pratiques de l'agriculture de conservation 
Bien que cette agriculture alternative ne bénéficie encore aujourd'hui ni d'encadrement institutionnel, ni d'aucune forme de reconnaissance auprès du consommateur, elle connaît un développement important à travers le monde dans des conditions pédoclimatiques et socioéconomiques très variées (Derpsch and Friedrich, 2009). L'ACS est en revanche encore très peu connue du grand public et mal accompagnée en France et en Europe où son adoption par les agriculteurs, bien que croissante, reste faible.

Pourtant, le développement significatif de l'ACS ces 40 dernières années notamment en Amérique du Sud, en Amérique du Nord et en Australie (Kassam et al., 2015) ainsi que l'intérêt scientifique récent pour cette agriculture, nous donnent aujourd'hui un aperçu des bienfaits, ou « externalités positives », qui lui sont associés, et ce au-delà du compartiment « sol ».

Une abondante littérature scientifique soutient aujourd'hui que favoriser l'application de pratiques agricoles respectueuses de la santé du sol, telles que celles employées en ACS, a non seulement des effets positifs sur les services écosystémiques associés au sol (I), mais également au-delà (II) y compris en termes de productivité. Même si les références sur l'ACS en France sont encore assez faibles, les récents résultats d'une étude française sur les effets de l'ACS sur différents services écosystémiques (III), démontrent son potentiel et soulignent d'autant plus l'intérêt de développer une meilleure protection des sols.

\section{L'ACS ET LA PROTECTION DES SOLS}

L'ACS présente des liens avec la santé des sols agricoles au moins à trois niveaux : par la réduction de sa sensibilité à l'érosion (A), la transformation des communautés du sol (B) et le stockage du carbone $(\mathrm{C})$.

\section{A. RÉDUCTION DU RISQUE ÉROSIF ET DES PERTES DE SOLS}

A l'origine même de l'apparition de l'ACS, la protection contre l'érosion est le principal objectif recherché par cette forme d'agriculture. Au-delà de limiter la perte pure et simple de sol sur les parcelles agricoles, réduire l'érosion a des impacts positifs tant sur la qualité de l'eau que sur la fertilité des sols, mais également 
des impacts indirects et globaux, sur le changement climatique par exemple.

Même si la réduction du travail du sol est souvent considérée comme la pierre angulaire de l'ACS, c'est bien la combinaison de ses trois principes qui génère les divers services portés à son crédit. La modification de la répartition de la matière organique dans le profil cultural est l'un des principaux impacts sur le sol de cette combinaison. La matière organique se concentre particulièrement en surface en ACS alors qu'elle est répartie plus ou moins uniformément dans le sol, de la surface à la ligne de labour (profil cultural) dans les systèmes conventionnels. La diminution du travail du sol, l'absence de labour ${ }^{3}$ notamment, dans ces systèmes en est la principale cause et la répartition de la matière organique s'apparente alors davantage à celle d'une prairie naturelle ou d'une forêt qu'à une parcelle travaillée. La couverture permanente du sol quant à elle, est la principale cause de l'augmentation de matières organiques en surface, souvent secondée par de plus gros amendements organiques dans ce type d'agriculture que dans des agricultures plus conventionnelles.

La matière organique représente moins de $10 \%$ des constituants d'un sol, elle est pourtant indispensable à son fonctionnement. Elle assure notamment sa cohérence, les particules de sol sont mieux arrimées entre elles lorsque la quantité d'humus (matière organique morte, décomposée et stable) est élevée. La modification de la répartition de la matière organique dans le profil de sol et son augmentation en surface améliorent ainsi la stabilité structurale du sol, diminuent le risque érosif et augmentent sa portance (Soane et al., 2012). De plus, au-delà de l'apport de matière organique qu'elle procure, la couverture permanente du sol en ACS, accentue ses effets positifs à l'égard de l'érosion (Labreuche et al., 2007; Soane et al., 2012) en offrant une protection physique contre la destructuration des agrégats de surface par les impacts de gouttes de pluie essentiellement. Cette stabilité limite ainsi la formation d'une croûte de battance, c'est-àdire la compaction du sol en surface (sur quelques millimètres de

3. Le labour est une technique de travail du sol, souvent la plus profonde (de 20 à $30 \mathrm{~cm}$ en France), consistant à retourner et mélanger l'ensemble de la couche arable pour l'ameublir, détruire les adventices et enfouir les résidus. C'est l'opération de base en Europe, elle est ensuite suivie d'interventions plus superficielles pour affiner la structure du sol et préparer le lit de semence. 
profondeur), à l'origine d'un ruissellement érosif des eaux de pluie. Ces effets s'observent tout particulièrement avec la pratique du semis direct (pratique de réduction maximale des perturbations du $\mathrm{sol}^{4}$ ), avec lequel l'évolution du sol est plus rapide (West and Post, 2002) qu'en TCS ${ }^{5}$. De plus, l'arrêt du labour en ACS permet non seulement une économie d'énergie (puissance de traction nécessaire moindre) mais aussi de travailler davantage en travers de la pente, aussi bien en TCS qu'en semis direct (selon les types d'outils, notamment de semoirs), réduisant par-là d'autant plus l'érosion aratoire dans les parcelles en coteaux où le ruissellement est déjà problématique.

Néanmoins, l'efficacité, en termes de réduction de l'érosion, des techniques de conservation des sols, est très variable selon la texture du sol. En effet, si sur des sols argileux, leur efficacité est la plus probante (Chichester and Richardson, 1992; Labreuche et al., 2007; Rhoton et al., 2002; Tebrügge and Düring, 1999), leur efficacité sur des sols sableux semble moindre (Quinton and Catt, 2004) et les résultats sur sols limoneux sont très variables, dépendant essentiellement d'autres paramètres comme des types de culture inclus dans la rotation.

La réduction du travail du sol en ACS est également à l'origine de transformations profondes de la structure du sol. La qualité physique du sol est améliorée par la formation d'une porosité biologique de surface qui garantit une meilleure infiltration et améliore ainsi la valorisation de l'eau de pluie et des éléments minéraux (en particulier le phosphore) par les cultures (Soane et al., 2012). Ces phénomènes sont lents à mettre en place et les premières années après le passage en ACS, on observe parfois une reprise en masse du sol, se traduisant par une augmentation de la densité apparente du sol sur les 20-25 premiers centimètres. Cela s'observe en particulier dans les systèmes

\footnotetext{
4. Le semis direct est une pratique qui consiste à abandonner toute forme de préparation du sol avant semis, de manière ponctuelle ou définitive. La seule perturbation mécanique du sol est celle générée par le semoir (spécifique à ce type de pratiques) sur quelques centimètres de profondeur lors de l'ouverture et la fermeture du sillon. Le semis direct est considéré comme la pratique phare de l'ACS, mais celle-ci nécessite la mise en place des deux autres principes que sont la couverture permanente du sol par des plantes maitrisées et leurs résidus, et l'allongement de la rotation.

5. Techniques Culturales Simplifiées : façons culturales basées sur la réduction des perturbations physiques des sols en vue de préparer le semis et/ou lutter contre les adventices. Elles excluent donc le labour (retournement du sol à l'aide d'une charrue) mais aussi généralement tout travail profond même sans retournement de la terre.
} 
mal maîtrisés, et se traduit par une aération réduite de l'horizon de surface, et un réchauffement du sol par conséquent ralenti au printemps, mais aussi par une augmentation de l'hydromorphie de surface. L'ensemble de ces phénomènes aboutit à un retard des semis de printemps. La porosité n'est en général reconstituée qu'après quelques années et se trouve être alors de nature biologique et non plus mécanique. Elle n'est en effet plus créée par des passages d'outils (fissurations et macroporosité verticale) mais principalement par l'activité biologique (macro- et microporosité interconnectées), notamment des vers de terre, ainsi favorisée. Dans les sols contenant plus de 15-20\% d'argile, les variations climatiques (alternance humectation/dessiccation, gel/dégel) agissent elles aussi sur la structure du sol par les changements de volume du sol qui créent des fissurations et donc de la porosité dans les agrégats. Il en résulte que la porosité biologique se caractérise, par rapport à la porosité mécanique, par une architecture différente et notamment par une interconnexion plus importante entre macro- et micropores. La porosité créée par les vers de terre anéciques (gros vers effectuant des parcours de direction générale verticale mais plus ou moins sinueux entre la surface du sol et ses horizons profonds situés à plus de 1 mètre), associée à un réseau très connecté de microporosité, augmente la réserve en eau facilement utilisable par les plantes ainsi que la conductivité hydraulique verticale, avec les horizons profonds, mais aussi horizontale, et favorise le drainage de l'eau (Soane et al., 2012). Le sol devient également moins sensible au tassement généré par les engins agricoles, sauf en conditions très humides, grâce à sa plus grande portance, elle-même générée par une plus forte cohésion des agrégats entre eux (Labreuche et al., 2007).

Le bouleversement physico-chimique du sol en ACS est ainsi étroitement lié à l'évolution des communautés biologiques qui l'occupent. La modification profonde de l'habitat qu'est le sol, entraîne en effet une réorganisation importante, bien qu'encore mal comprise, des communautés inféodées au sol (l'édaphon), ellesmêmes en partie responsables de l'évolution de la structure du sol et de la disponibilité des éléments nutritifs.

\section{B. FAVORISER LA BIODIVERSITÉ DES SOLS ET SES FONCTIONS ÉCOLOGIQUES}

De manière générale, la composition et les structures des communautés du sol sont modifiées par l'ACS, en particulier à l'interface entre le sol et les résidus de culture laissés à sa surface 
(Tebrügge and Düring, 1999). Les habitats du sol sont modifiés, les ressources spatialement redistribuées et, plus directement, certains organismes qui auraient été immédiatement affectés par le travail du sol (destruction mécanique, dessèchement en surface et exposition à des prédateurs), sont protégés de ces risques en ACS. Ces modifications vont influencer toute la chaîne trophique des agroécosystèmes, aussi bien en termes d'abondance ou de diversité en espèces qu'en termes de distribution et d'activité, impactant de ce fait de nombreux services écosystémiques : nutrition des plantes, structuration du sol, contrôle des organismes bioagresseurs, stockage du carbone, émission de gaz à effet de serre, etc. (Vieublé, 2015).

Globalement, l'activité biologique du sol augmente en surface grâce à l'ACS, mais elle peut être réduite en profondeur en raison de l'absence d'enfouissement des matières organiques. De nombreuses études ont été, et sont encore, menées sur l'impact de l'ACS sur l'édaphon et montrent généralement un effet positif de ces pratiques bien que de fortes disparités soient observées entre les différentes tailles d'organismes (Kladivko, 2001; van Capelle et al., 2012; Wardle, 1995). La macrofaune est significativement favorisée par l'ACS, par rapport à la méso- et la microfaune (Kladivko, 2001; Wardle, 1995). Les organismes de grande taille sont en effet plus durement impactés par la perturbation mécanique dans les systèmes conventionnels (mort des individus, exposition à la prédation, etc.) et profitent de la redistribution des ressources et des habitats en ACS. En particulier, l'ACS a un impact positif avéré sur les vers de terre, véritables architectes du sol et indispensables à sa structuration naturelle. L'absence de perturbations mécaniques et l'augmentation de la teneur en matières organiques en surface, modifient la répartition dans le profil de sol des ressources et des conditions d'habitat (flux d'air et d'eau) de l'édaphon. Le paillis, ou « mulch », ainsi constitué en surface par les débris végétaux va fournir un abri et une source de nourriture pour la faune de surface. Le mulch ralentit également l'assèchement du sol en fin de printemps et retarde le gel en fin d'automne, permettant ainsi des transitions entre saisons plus douces, favorables au développement des vers de terre. Les vers peuvent alors se nourrir et se reproduire plus longtemps et probablement mieux s'acclimater aux changements de saison. De nombreuses études et observations de terrain confirment par ailleurs que le nombre de vers de terre dans une parcelle agricole augmente avec la diminution de l'intensité du travail du sol 
(Kladivko, 2001). Les vers épigés et anéciques, qui s'alimentent tous en surface, sont particulièrement favorisés par les pratiques maintenant les résidus à la surface du sol. Il n'est par ailleurs pas rare qu'en système conventionnel, plus aucun ver épigé (déjà rares dans les agrosystèmes) ou anécique ne soit comptabilisé. Des espèces comme Lumbricus terrestris, ver anécique commun en Europe, peuvent complètement disparaitre suite à de nombreuses années en labour du fait de leur taux de reproduction très bas et d'une grande sensibilité à la perturbation du sol et à l'enfouissement des résidus. La re-colonisation de la parcelle par les vers de terre se faisant par ses abords, dans les régions où l'agriculture a été très intensive pendant de nombreuses années, il devient difficile de les voir réapparaitre même après un passage en ACS (Kladivko, 2001).

La microfaune et la microflore quant à elles, ne sont pas systématiquement favorisées dans leur diversité en ACS. En revanche, l'évolution physique et chimique du sol en ACS, influe significativement sur la répartition des espèces et sur la balance entre bactéries et champignons par rapport aux systèmes en labour (Doran, 1980; Kladivko, 2001). Ces organismes étant étroitement liés à la fertilité des sols, à la capacité des plantes à prélever les éléments nécessaires à leur développement et aux risques phytosanitaires, ces équilibres sont d'une importance primordiale à la stabilisation de la production (Ishaq, 2017).

Suivant la répartition de la matière organique, une forte stratification verticale des micro-organismes du sol se met en place en semis direct, aussi bien en termes de quantité que d'activité, par rapport à la répartition plus homogène dans le profil d'un sol labouré (Andrade et al., 2003). En semis direct, les champignons dominent de $0-5 \mathrm{~cm}$ là où les bactéries dominent en labour (Frey et al., 1999). La biomasse et l'activité microbienne dans la couche $0-10 \mathrm{~cm}$ sont significativement plus élevées qu'en labour, mais deviennent inférieures ou égales dans les couches sous-jacentes (Wright et al., 2005). Cela a des répercussions sur la vitesse de minéralisation du carbone et de l'azote, et donc leur disponibilité pour les plantes. Dans les couches superficielles, la minéralisation est plus élevée en ACS du fait de l'activité microbienne plus élevée. Cependant, dans les couches profondes ( $>10$ ou $15 \mathrm{~cm})$, l'inverse se produit et la vitesse de minéralisation du carbone et de l'azote est alors bien inférieure à celle en systèmes labourés (Grigera et al., 2007; Pekrun et al., 2003). A cela s'ajoute une évolution globale de la 
communauté de bactéries et de champignons vers des espèces plus adaptées aux nouvelles qualité et répartition de la matière organique. L'ACS stimule les eubactéries actinomycètes copiotrophes (adaptées à de hauts niveaux de nutriments, particulièrement de carbone) au développement lent et impliquées dans la dégradation progressive de la matière organique fraiche. Elle stimule également les bactéries Azotobacter et Nitrospira, toutes fortement impliquées dans le cycle de l'azote $^{6}$, les unes comme les autres indicatrices d'un environnement stable. Bien que les bactéries prédominent et présentent une diversité génétique plus importante en labour et travail du sol intensif (Kladivko, 2001), les bactéries nitrifiantes et anaérobies sont plus diversifiées en semis direct (Drijber et al., 2000; Ibekwe et al., 2002), rendant ainsi possible la fourniture d'azote minérale à partir de matières organiques humifiées du sol, même situées dans des horizons ou compartiments pauvres ou dépourvus d'oxygène. L'ACS stimule également la présence de champignons ascomycètes qui dégradent la matière organique fraiche peu récalcitrante ${ }^{7}$, et de champignons mycorhiziens (Drijber et al., 2000) en particulier dans les premiers centimètres du sol grâce à la plus forte humidité sous le mulch et à la non perturbation mécanique (Spedding et al., 2004). La colonisation des racines des plantes par les mycorhizes est alors plus importante en semis direct (McGonigle et al., 1999), favorisant la prospection racinaire et donc leur capacité à capter l'eau et les nutriments, tout en renforçant les défenses naturelles des plantes (Selosse et al., 2004). En labour ou travail du sol intensif en revanche, ce sont en particulier des champignons basidiomycètes, capables de dégrader la matière organique récalcitrante, qui sont favorisés. La matière organique fraiche étant rapidement consommée par les bactéries, plus nombreuses dans ces systèmes, ces champignons dégradent cette matière organique plus stable, remettant en cause la durabilité du système puisque les matières organiques du sol à l'origine de sa meilleure structure et d'un stockage à long terme du carbone dans le sol, s'amenuisent peu à peu du fait de ces pratiques. Globalement, les communautés bactériennes et fongiques favorisées en ACS permettent une dégradation plus lente de la matière organique

6. Les Azotobacter fixent le diazote gazeux dans des molécules organiques et les Nitrospira transforment les ions nitrites en ions nitrates.

7. La matière organique récalcitrante est la part très stable (riches en sucres complexes comme la lignine et les tanins) qui améliore les propriétés physiques du sol et stocke le carbone sur le long terme (fonction « puits de carbone » des sols).) 
et une fourniture plus progressive des éléments minéraux pour la plante tout au long de son développement. De plus, le rapport champignons/bactéries passe de 1 pour 1 en parcelle labourée à 3 pour 1 en parcelle sous ACS (Brady and Weil, 2008), tendant ainsi à se rapprocher des écosystèmes naturels où la domination fongique peut atteindre un ratio de 100 pour 1 (sols forestiers). De nombreuses espèces de champignons se développent en effet sous forme de réseaux de filaments (mycélium), réseaux qui sont particulièrement endommagés par le travail mécanique du sol, réduisant ainsi significativement la quantité et la diversité de ce type de champignons en systèmes conventionnels. Or, ces réseaux mycéliens jouent un rôle clé dans la stabilité structurale du sol (Guggenberger et al., 1999) et dans le stockage à long terme du carbone (Bailey et al., 2002) notamment.

Enfin, la mésofaune (insectes, nématodes, collemboles, etc.) est la part de l'édaphon pour laquelle il est encore aujourd'hui difficile de conclure sur l'effet des pratiques mais également sur les conséquences pour les processus, et donc services écosystémiques, qui lui sont associés. En 1995, David A. Wardle (Wardle, 1995) compile les résultats de 106 études menées jusqu'alors et souligne que de fortes variations sont observées d'une étude à l'autre, en particulier concernant cette faune de taille intermédiaire, selon les intensités de travail du sol et d'enfouissement pratiqués, les périodes de l'année, la culture étudiée et les conditions de sol et de climat. De plus, il souligne l'importance des pratiques associées à la réduction du travail du sol, or la définition de l'ACS à l'époque, et encore aujourd'hui dans une certaine mesure, ne permettait pas d'identifier clairement les exploitations pratiquant réellement l'ACS dans le respect de ses trois principes. Les études plus récentes, menées cette dernière décennie, confirment toujours les conclusions de David Wardle. Christine Van Capelle et al., qui ont compilé en 2012 (van Capelle et al., 2012) les études allemandes sur le sujet, concluent que les impacts, en particulier à long terme, sur la biodiversité du sol sont toujours peu compris, notamment l'interaction entre pratiques, texture du sol et type de culture.

\section{Stockage du Carbone en ACS}

L'agriculture est comme la plupart des activités humaines, en partie responsable des émissions de gaz à effet de serre et donc des pertes 
de carbone fossile ${ }^{8}$ vers l'atmosphère où le carbone s'accumule sous forme minérale $\left(\mathrm{CO}_{2}\right.$, le gaz carbonique), accentuant pollutions et réchauffement climatique (GIEC, 2014). Les sols peuvent ainsi perdre par l'agriculture intensive jusqu'à $75 \%$ de leur carbone organique (Lal, 2011), perte qu'il faudra alors compenser par des apports exogènes, parfois coûteux, afin de maintenir la productivité. Pourtant, à l'état naturel, les sols sont un indispensable puits de carbone et on estime aujourd'hui qu'une gestion appropriée des sols dans les agroécosystèmes peut permettre un potentiel de re-stockage de 1,2 à 3,1 milliards de tonnes de carbone par an (Lal, 2011; Paustian et al., 2016). Ce stockage concerne particulièrement l'horizon de surface mais on sait désormais qu'il s'opère également dans les zones plus profondes, au-delà de $30 \mathrm{~cm}$ (de Moraes Sa and Lal, 2009; Mulder et al., 2016; Plaza-Bonilla et al., 2016). Les apports de matières organiques via les amendements, les résidus de culture ou les couverts végétaux sont le principal vecteur de ce stockage. Ils permettent ainsi de stocker 0,3 à 0,5 tonne de carbone par hectare et par an, que cela soit avec ou sans travail du sol (Dimassi et al., 2014; Lal, 2015a). Et ces chiffres peuvent atteindre 0,5 à 0,9 si l'ACS est appliquée dans ses trois principes (Olson et al., 2014; Powlson et al., 2016). John M. Baker et al. (Baker et al., 2007) estiment ainsi en 2007 que 25 milliards de tonnes de carbone pourraient être re-stockées en 50 ans si toutes les terres arables passaient en ACS.

On remarquera tout particulièrement ici que c'est bien la synergie des trois piliers de 1'ACS qui permet ce stockage (Lal, 2015b; Pisante et al., 2015). En effet, le non travail du sol, bien qu'important pour la préservation structurelle des agrégats du sol, n'a finalement qu'un effet marginalement positif sur le stockage du carbone (de Moraes Sa and Lal, 2009; Powlson et al., 2014) s'il n'est pas associé à l'utilisation de couverts végétaux, à la restitution des résidus et au raisonnement de la rotation. L'utilisation de variétés ou d'espèces avec une importante masse racinaire dans la rotation permet par exemple de déposer le carbone plus en profondeur dans le profil, là où le turnover des éléments est moins important, favorisant ainsi un stockage pérenne du carbone (Kell, 2012; Paustian et al., 2016). Avoir recours aux couverts végétaux pendant les périodes d'interculture permet une séquestration du carbone tout au long de

8. Carbone stable dont les processus de stockage dans le sous-sol se réalisent sur des temps géologiques. 
l'année et, avec des espèces bien choisies, permet également une synergie avec le cycle de l'azote (Lal, 2015c).

En plus de participer à la réduction des gaz à effet de serre, le stockage de carbone dans les sols agricoles contribue à l'amélioration qualitative et quantitative du cycle de l'eau, à la restauration des sols et des écosystèmes et la biodiversité qui leur est associée et à la sécurité alimentaire globale. En effet, l'augmentation du carbone organique dans les sols participe à sa qualité et par là-même à la production de denrées alimentaires. Dans les pays en développement, un gain d'une tonne de carbone par hectare et par an, s'accompagnerait d'un gain moyen de 32 millions de tonnes de céréales par an (Lal, 2011).

\section{L'ACS ET LA CONSERVATION DE SERVICES AU-DELÀ DU SOL}

Alors que les effets positifs des pratiques de 1'ACS sur la santé des sols sont attendus, les impacts indirects de pratiques de préservation des sols sont tout aussi importants. L'ACS participe ainsi également à répondre aux enjeux liés au changement climatique (A), à la lutte contre les bioagresseurs des cultures (B) et au maintien de la productivité agricole $(\mathrm{C})$.

\section{A. LEVIER FACE AU CHANGEMENT CLIMATIQUE}

L'impact des activités humaines sur le climat implique aujourd'hui deux impératifs : atténuer notre impact sur le climat et nous adapter aux changements engendrés. Le changement climatique a notamment un impact important sur l'agriculture, car il modifie la phénologie des plantes cultivées, altère leur physiologie et leur productivité et complique la gestion de l'eau. L'ACS, du fait de son impact sur le sol en particulier, contribue aussi bien à l'atténuation qu'à l'adaptation au changement climatique (Pisante et al., 2015).

Comme nous venons de l'évoquer, l'ACS présente un fort potentiel pour le stockage du carbone atmosphérique et donc pour l'atténuation des risques associés aux gaz à effet de serre comme le $\mathrm{CO}_{2}$ sur le réchauffement climatique. A cela peut s'ajouter le fait qu'en ACS, la réduction du nombre de passages d'outils de travail du sol et une gestion plus intégrée de la fertilisation grâce à la matière organique et aux couverts de légumineuses surtout, engendre de moindres 
consommations d'énergies fossiles et émissions de gaz à effet de serre (Holland, 2004). La consommation de carburant en ACS est ainsi diminuée de $60 \%$ par rapport à des systèmes conventionnels (SoCo, 2009) et on constate également une diminution des émissions de $\mathrm{N}_{2} \mathrm{O}$ et de $\mathrm{CH}_{4}$, autres gaz à effet de serre, après quelques années d'ACS bien conduite (Dendooven et al., 2012; Palm et al., 2014; Six et al., 2004) notamment du fait de l'évolution de l'activité bactérienne du sol.

L'ACS est également elle-même plus résiliente face au changement climatique, compte tenu de la résilience de sa productivité en contexte de stress hydrique (Pittelkow et al., 2014). Les changements des propriétés physiques, chimiques et biologiques du sol associés à l'ACS permettent d'améliorer la fourniture en eau et stabilisent la production en atténuant l'effet d'épisodes de sècheresse ou d'intempéries violentes (Holland, 2004), susceptibles de devenir plus fréquents à l'avenir. Cette résilience est particulièrement imputable aux bénéfices tirés des apports de matières organiques au sol (Song et al., 2015), à la stabilité des agrégats de surface qui empêche la fermeture du sol en cas de pluie forte, aux résidus qui améliorent le stockage de l'eau et ralentissent le ruissellement, et à la micro- et mésoporosité biologiques qui maximisent la rétention de l'eau pour les épisodes de sècheresse (Pisante et al., 2015).

\section{B. RÉGULATIONS BIOLOGIQUES}

De nombreux services dits « de régulation » (au sens du MEA, 2005) sont à la base de la production agricole. La valeur de la production alimentaire mondiale assurée par la pollinisation animale, par exemple, a été estimée à 153 milliards d'euros en 2005 (Gallai et al., 2009; Klein et al., 2007), les insectes pollinisateurs étant garants de la reproduction de $84 \%$ des plantes cultivées pour l'alimentation humaine en Europe, 65 à $70 \%$ au niveau mondial (Williams, 1994), soit en volume, $35 \%$ de la production alimentaire mondiale (Klein et al., 2007). De même, le biocontrôle des ravageurs par leurs ennemis naturels est l'un des plus importants services écosystémiques intrants ${ }^{9}$ des agroécosystèmes (Fiedler et al., 2008; Gurr et al., 2003; Wilby and Thomas, 2002). Ces auxiliaires prédateurs et parasitoïdes sont responsables de $50 \%$ du contrôle des ravageurs dans le monde,

9. V. Glossaire. 
contre $40 \%$ pour les facteurs génétiques et seulement $10 \%$ pour les pesticides (Pimentel and Burgess, 2014). A l'échelle mondiale, ils permettent à eux seuls de réduire de 100 milliards $\$$ an les pertes liées aux ravageurs (Pimentel et al., 1997).

L'usage d'insecticides et, dans une moindre mesure, de fongicides sur les cultures nuit aux insectes bénéfiques, comme les pollinisateurs ou les ennemis naturels des ravageurs de culture (Lavorel and Sarthou, 2008; Oerke, 2006). Dans une logique de protection intégrée des cultures et de contrôle biologique par conservation, l'usage de ces intrants représente donc la pratique de base à éviter afin de promouvoir l'abondance et la diversité des ennemis naturels dans les cultures (Ehler, 2006) et le contrôle biologique des ravageurs qu'ils assurent (Howarth, 2000; Jonsson et al., 2008). L'impact positif de l'agriculture biologique, interdisant l'usage de produits biocides de synthèse, sur ces organismes est aujourd'hui avéré (Winqvist et al., 2012).

En revanche, le potentiel bénéfice de la préservation des sols par l'ACS vis-à-vis des régulations biologiques s'y déroulant, est beaucoup moins connu et étudié. Pourtant, l'intensité du travail du sol, en particulier le labour (c'est-à-dire l'inversion du sol), a des effets négatifs bien documentés sur les arthropodes du sol (Kladivko, 2001; Legrand et al., 2011; Shearin et al., 2007; Witmer et al., 2003). Le travail du sol a par ailleurs été utilisé pendant des siècles pour contrôler les ravageurs dans les champs, notamment les ravageurs du sol. En ce qui concerne les insectes auxiliaires, plusieurs études ont montré une plus grande diversité de prédateurs, notamment carabes et araignées, dans les parcelles en semis direct ou en non-labour que dans celles subissant régulièrement un labour (Holland and Luff, 2000; Kosewska et al., 2014; Marti and Olson, 2007), les espèces de grande taille étant une fois encore les plus touchées par l'effet du travail du sol (Hatten et al., 2007). La présence de résidus de culture en surface et d'un couvert végétal permanent offrent un environnement plus complexe, apprécié par les araignées (Rypstra et al., 1999), des abris et un microclimat tamponné favorable au développement de nombreux arthropodes à différents stades de leur cycle de vie et périodes de l'année (Roger-Estrade et al., 2010). A cela s'ajoute la présence de proies alternatives, favorisées par l'augmentation de la matière organique, de résidus de cultures et de graines d'adventices non enfouies (Holland, 2004). 
De plus, peu de recherches ont porté sur les effets de la gestion du sol sur les ennemis naturels aériens et moins encore sur les polinisateurs. Pourtant, des effets bénéfiques similaires du non-labour ont été montrés pour une espèce d'abeille sauvage pollinisatrice des courges (Shuler et al., 2005), pour les coccinelles (Marti and Olson, 2007), les guêpes parasitoïdes Trichogrammatidés (Sharley et al., 2008), les coléoptères volants, les larves de syrphes et les chrysopes (Tamburini et al., 2016). Certaines de ces espèces, bien qu'a priori non inféodées au sol, profitent des évolutions de l'agroécosystème en ACS. En effet, certaines espèces d'abeilles ou de syrphes (Diptères, Syrphidés), celles qui nidifient ou hivernent dans le sol parfois au sein même des parcelles agricoles (Raymond et al., 2014), profitent de l'absence de perturbation. D'autres tirent profit plus indirectement du passage en ACS, par la création d'habitats, la plus grande diversité d'adventices (souvent plus présentes et tolérées en ACS), ou encore par la modification physiologique des plantes hôtes qui ont poussé dans un sol à la biodiversité riche, notamment en bactéries fixatrices d'azote et en mycorhizes, renforçant leurs défenses naturelles ou modifiant leur attractivité pour les pollinisateurs notamment (Barber and Gorden, 2015).

Concernant l'efficience du contrôle biologique des ravageurs des cultures et leur impact sur les cultures, il est constaté qu'ils sont respectivement meilleure (Tamburini et al., 2016) et équivalent à moindre (Basch et al., 2015; Kesavan and Malarvannan, 2010; Kutcher et al., 2011) en ACS par rapport à un mode de culture conventionnel. Beaucoup d'études sur l'effet du travail du sol ne prennent pas en compte les interactions avec les autres pratiques de l'ACS, or la maitrise des bioagresseurs dans ce type de systèmes ne peut être atteinte qu'au moyen d'une bonne utilisation de la rotation en particulier, notamment contre les maladies (Leake, 2003). Les trois piliers de l'ACS sont en ce sens indissociables pour assurer le maintien voire l'amélioration des services de régulation (RogerEstrade et al., 2010). Certains cas restent par ailleurs problématiques comme les limaces, largement reconnues et documentées comme difficilement gérables sans travail du sol, en particulier les premières années.

\section{Performances agronomiques et économiques de l'ACS}

On estime qu'à travers le monde, tous types de climats et de cultures confondus, l'ACS présente une baisse de rendement de l'ordre 
de 2,5\% par rapport à l'agriculture conventionnelle (Pittelkow et al., 2014) ${ }^{10}$. En revanche, dans un contexte de stress hydrique, les rendements observés en ACS sont en moyenne plus élevés qu'en agriculture conventionnelle (Pittelkow et al., 2014). On constate ainsi en climats secs une augmentation des rendements de l'ordre de 50 à $100 \%$ selon Oihane Fernández-Ugalde et al. (FernándezUgalde et al., 2009) et entre 20 à $120 \%$ selon Amir Kassam et al. (Kassam et al., 2012) par rapport à l'agriculture conventionnelle.

A l'échelle européenne, des différences notables s'observent entre pays. Au nord du continent, les rendements sont globalement moins bons en ACS et chutent de 6,9\%. Le sud en revanche (incluant la France) présente des rendements supérieurs d'environ $13 \%$ par rapport 1'agriculture conventionnelle (Basch et al., 2015). Les niveaux de rendement mesurés en ACS sont par ailleurs particulièrement dépendants de l'ancienneté du système et tendent à augmenter au fil des années (Pittelkow et al., 2014).

Au-delà des rendements, différents auteurs reconnaissent que le profit économique de 1'ACS est plus élevé (Sturny and Chervet, 2015; Tebrügge and Böhrnsen, 1997) par rapport à un mode de production plus conventionnel du fait de frais de main d'œuvre réduits de 50 à $75 \%$ (SoCo, 2009), de frais de carburants réduits de $60 \%$ (SoCo, 2009) et de frais d'entretien de matériel réduits de $80 \%$ (Freixial and Carvalho, 2010). L'amélioration de la teneur en carbone organique dans les sols et l'incorporation de légumineuses dans la rotation ajoutent à cela la possibilité de réduire également les coûts de fertilisation azotée après quelques années (Carvalho et al., 2012). Tout ceci abaisse le seuil de rentabilité (niveau de rendement) à partir duquel l'exploitation est économiquement viable. Au Portugal, pour une ferme de 500 ha, ce seuil de rentabilité (calculé sur la marge nette) a ainsi été estimé à 14,3 quintaux de blé par ha en labour contre 11,3 en ACS (Marques and Basch, 2002).

10. A la parution de cette étude, un mouvement de contestation de la part des «farmers » d'Amérique du Nord s'est levé pour critiquer les estimations faites par les organismes de recherche et les instituts techniques, sous-estimant selon eux les performances de l'ACS. "29 Reasons Why Many Growers Are Harvesting Higher No-Till Yields in Their Fields Than Some University Scientists Find In Research Plots" http://www.no-tillfarmer.com/ articles/4038 
En revanche, la principale limite à l'abandon ou à la forte diminution du travail du sol est que cela enlève aux producteurs leur principal moyen de lutte contre les adventices. Le recours au travail du sol pour la destruction mécanique des adventices ou la pratique du faux semis, par exemple, est en effet la principale alternative à l'usage d'herbicides, elle lui est souvent préférée pour son efficacité et son moindre coût. De plus, sans travail du sol, les graines restent plus facilement en surface et la présence d'un mulch permanent modifie les conditions de germination, favorables à certaines espèces, mais limitantes à la germination de la plupart des espèces pour lesquelles une induction lumineuse est indispensable. Certaines graines laissées en surface sont également consommées ou endommagées par la biodiversité souvent plus riche en ACS et certaines adventices sont désavantagées par ces conditions particulières. On observe ainsi en ACS une sélection d'un cortège d'adventices différentes de celles qui auraient été sélectionnées en conventionnel (Holland, 2004). Al'heure actuelle, en l'absence de travail du sol, le contrôle chimique des adventices est encore l'option la plus efficace et donc la plus répandue.

Le passage à l'ACS demande ainsi un changement de paradigme radical en ce qui concerne la gestion des adventices. Or les moyens humains, les outils et même les stratégies de lutte contre les adventices en ACS sont encore trop peu disponibles pour accompagner la conversion (Singh et al., 2015). En pratique, le passage en ACS s'accompagne à l'heure actuelle d'un usage accru d'herbicides les premières années, durant une phase de transition principalement liée à l'apprentissage par l'agriculteur et à la maturation du système (transformation biotique et abiotique du sol notamment). En revanche, après quelques années d'ACS bien conduite, adoptant une démarche de gestion intégrée des adventices en combinant méthodes chimiques et non chimiques (essentiellement destruction mécanique, rotation allongée, couverts végétaux pendant les intercultures), on constate une diminution de la levée d'adventices après semis (Gupta and Seth, 2007; Singh et al., 2015) et une diminution du recours aux herbicides, qui devient équivalent voire inférieur aux systèmes conventionnels (Sturny and Chervet, 2015). Aussi, Farooq et Siddique placent dans leur livre "Conservation Agriculture » (Farooq and Siddique, 2015b), le contrôle des mauvaises herbes comme « 4ème pilier » de l'ACS, soulignant par-là l'importance de cette question pour ces systèmes. L'utilisation habile des couverts végétaux est un levier particulièrement déterminant pour la gestion 
des adventices en ACS, mais l'expertise sur les couverts, tant de la part des agriculteurs que des conseillers, est encore trop rare et peu adaptée aux variations des conditions locales.

\section{UN EXEMPLE FRANÇAIS : LES CONCLUSIONS DU RÉSEAU D'OBSERVATION SERACC (SERVICES ÉCOSYS- TÉMIQUES EN AGRICULTURE CONVENTIONNELLE ET DE CONSERVATION)}

Entre 2013 et 2016, une étude comparant simultanément l'expression de dix-sept services écosystémiques (Fig. 2) en ACS à celle en agricultures biologique et conventionnelle (avec des intensités variables de travail du sol) a été menée par l'INRA de Toulouse, en collaboration avec un réseau d'une cinquantaine d'agriculteurs dans trois départements autour de Toulouse (Chabert, 2017).

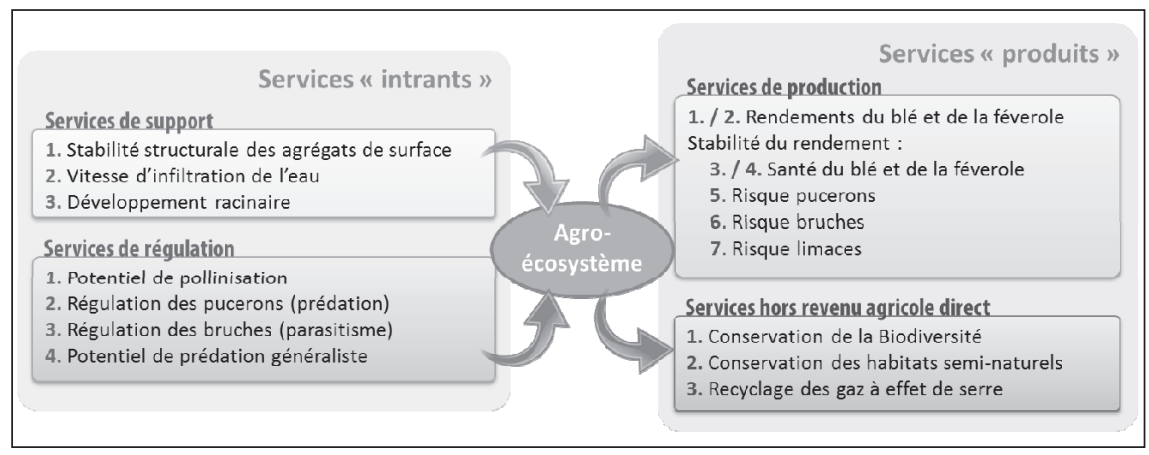

Figure 2.

Les 17 services étudiés dans un cadre de SERACC. Sept services « intrants" (" services de régulation et de maintenance » de la classification CICES ${ }^{11}$ ) dont quatre services de régulation et trois services de support, sept services de production contribuant au revenu agricole (« services d'approvisionnement» de la classification CICES) et trois services produits hors revenu agricole direct (« services de régulation et de maintenance » ou «culturels » de la classification CICES). 
Ce cas d'étude français, réalisé sur deux années culturales et pour deux cultures d'hiver (blé tendre d'hiver et féverole d'hiver), a permis de confirmer un certain nombre des connaissances générales concernant la relation entre l'ACS et les services écosystémiques en les contextualisant sur notre territoire.

Dans cette région de coteaux, aux sols argilo-calcaires parfois limoneux, les effets positifs de l'ACS, notamment du semis direct, sur la stabilité structurale des agrégats de sol ont pu être confirmés. Sous l'effet de pluies violentes, les gouttes d'eau n'arrachent pas ou très peu les particules de sol à la surface des parcelles en semis direct en hiver, contrairement aux sols en labour. En revanche, les pratiques de travail simplifié (TCS) ont montré des résultats très variables, soulignant la sensibilité au type de sol de ces pratiques intermédiaires. Néanmoins, le fait qu'en ACS l'eau soit potentiellement mieux retenue dans les sols, implique que ces derniers sont plus saturés en hiver et ils ont présenté une capacité d'infiltration des eaux de pluie limitée, potentiellement source d'un ruissellement hivernal et printanier plus important qu'en système avec labour. Il est toutefois légitime de penser qu'avec la très forte différence de stabilité structurale entre un sol labouré et un sol en semis direct, la présence d'un couvert et d'un mulch, combinée aux irrégularités du sol non travaillé (ne présente pas de sillons profonds et réguliers, souvent dans le sens de la pente), l'impact du ruissellement soit fortement amoindri en ACS par rapport à une agriculture plus conventionnelle, du fait d'une vitesse de ruissellement plus faible et de moindres pertes de sol.

L'analyse des performances agro-environnementales des exploitations du réseau SERACC a également confirmé le potentiel de l'ACS à réduire les impacts de l'agriculture sur les émissions de carbone, par la diminution des consommations de carburants, une utilisation plus raisonnée des intrants et notamment des fertilisants et la présence d'infrastructures agroécologiques. Certaines exploitations pratiquant le semis direct ou les TCS en agriculture biologique, présentent le potentiel de restocker jusqu'à l'équivalent de $200 \%$ de leurs émissions.

En ce qui concerne les régulations biologiques, les observations sur le réseau SERACC sont à l'image de la littérature internationale, très variables. Pour les trois cas de régulations étudiés, l'impact positif de l'ACS sur ces services n'a pu être significativement montré que pour 
le cas du puceron des épis. Néanmoins, les niveaux de régulation observés en ACS étaient comparables à ceux observés en agriculture biologique. L'ACS présente donc, dans la région, un potentiel d'amélioration de ces services à la hauteur de ce que peut offrir actuellement l'agriculture biologique, reconnue pour ses meilleures régulations biologiques que les systèmes conventionnels. De plus, le réseau d'observation SERACC a également permis de mettre en avant l'importance des bords de parcelles, lisières, bandes enherbées, haies, pour l'hivernation de nombreux ennemis naturels. Or, il s'est avéré que sur le réseau, les exploitations en agriculture biologique qui appliquaient également les principes de l'ACS (avec seulement un travail du sol superficiel) présentaient systématiquement une plus importante diversité en infrastructures agroécologiques. Ces producteurs, engagés dans une démarche agroécologique holistique, via la suppression d'intrants de synthèse, la réduction du travail du sol et la diversification, ont tout naturellement inclus dans leur diversification les espaces non-productifs. Ces habitats permettent ainsi le maintien d'une diversité d'ennemis naturels et donc un potentiel de régulation accru autour de ces parcelles, même si, comme nous l'a démontré la régulation des bruches, certains pourraient être désavantagés par cette diversification.

Malgré une potentielle augmentation du nombre de certains ravageurs comme les limaces ou les bruches, la productivité a été en moyenne maintenue en ACS par rapport à l'agriculture conventionnelle, avec une différence moyenne de $-0,5 \mathrm{q} / \mathrm{ha}$ en blé et $+5 \mathrm{q} / \mathrm{ha}$ en féverole. De plus, aucune tendance marquée pour le risque de maladies cryptogamiques n'a pu être montrée. Cependant, une forte variabilité a été observée en ACS et tous les systèmes ne se valent pas en termes de productivité. Sur l'ensemble du réseau, les plus hauts rendements comme les plus bas ont ainsi été observés en ACS. S'il n'est pas encore possible de conclure sur les tenants de cette variabilité, il semble toutefois que la maturité du système et sa maîtrise par le producteur, toutes deux garantes du rétablissement et du maintien de la santé du sol, en soient des facteurs déterminants.

Par ailleurs, ce réseau d'étude avait l'avantage de fournir une comparaison de l'ACS non seulement à une agriculture plus conventionnelle, mais également à une autre forme de systèmes agroécologiques : l'agriculture biologique. Cette dernière est reconnue pour réduire le transfert de polluants vers l'eau et les sols, favoriser le stockage du carbone par la matière organique, améliorer 
la qualité physicochimique et biologique du sol, émettre moins de $\mathrm{CO}_{2}$ et favoriser la biodiversité en oiseaux, plantes et insectes, en particulier les prédateurs (Bengtsson et al., 2005; Gomiero et al., 2011). Elle est cependant parfois décriée pour des pertes de productivité pour certaines cultures, pertes que peut engendrer l'abandon de la fertilisation azotée minérale et des traitements phytosanitaires de synthèse. Les observations faites sur le réseau SERACC tendent à confirmer ces affirmations. Globalement, la conservation de la biodiversité et des habitats semi-naturels était meilleure en agriculture biologique, comme en ACS, par rapport à l'agriculture conventionnelle. Cependant, la sensibilité à l'érosion s'est révélée bien plus importante en agriculture biologique qu'en ACS, mais également qu'en agriculture conventionnelle. De plus, si les rendements en féverole étaient peu impactés en agriculture biologique, les rendements en blé furent quant à eux significativement inférieurs $(-13,8 \mathrm{q} / \mathrm{ha}$ en moyenne par rapport à l'agriculture conventionnelle). En particulier, les exploitations qui ont présenté les plus bas niveaux de rendement étaient celles appliquant à la fois les principes de l'ACS et de l'agriculture biologique. En effet, si la combinaison de ces deux approches n'est pas sans vertu d'un point de vue écologique, la productivité de ce type de système est encore aujourd'hui trop difficile à assurer pour en garantir la durabilité.

Dans l'ensemble, cette étude est une illustration détaillée du potentiel de l'ACS pour s'affranchir de l'antagonisme apparent entre productivité et performances environnementales. L'étude a également révélé une grande variabilité de l'expression des services écosystémiques sous ACS, variabilité pouvant être attribuée principalement à l'immaturité du système, en termes d'équilibres écologiques et d'expertise de l'exploitant, ce type d'agriculture souffrant encore aujourd'hui d'un manque important d'accompagnement technique. 


\section{Bibliographie}

Andrade, D.S., Colozzi-filho, A., Giller, K.E., 2003. The Soil Microbial Community and Soil Tillage. In : Soil Tillage in Agroecosystems (A. El Titi, ed.), CRC Press, pp. 51-81.

Bailey, V.L., Smith, J.L., Bolton, H.J., 2002. Fungal-to-bacterial ratios in soils investigated for enhanced $\mathrm{C}$ sequestration. Soil Biology \& Biochemistry, 34, 997-1007.

Baker, J.M., Ochsner, T.E., Venterea, R.T., Griffis, T.J., 2007. Tillage and soil carbon sequestration-What do we really know? Agriculture, Ecosystems \& Environment, 118, 1-5.

Barber, N.A., Gorden, N.L.S., 2015. How do belowground organisms influence plant-pollinator interactions? Journal of Plant Ecology, 8, 1-11.

Basch, G., Friedrich, T., Kassam, A., Gonzalez-Sanchez, E., 2015. Conservation Agriculture in Europe. In: Conservation Agriculture (M. Farooq, K.H.M. Siddique, eds.), Springer International Publishing, Switzerland, pp. 357-388.

Bengtsson, J., Ahnstrom, J., Weibull, A.C., 2005. The effects of organic agriculture on biodiversity and abundance: a metaanalysis. Journal of Applied Ecology, 42, 261-269.

Brady, N.C., Weil, R.R., 2008. The nature and properties of soils. Pearson Prentice Hall, New Jersey, 621 p.

Carvalho, M., Basch, G., Calado, J.M.G., Barros, J.F.C., 2012. Long term effect of tillage system and crop residue management on soil carbon content of a Luvisol under rainfed Mediterranean conditions. Agrociencia, 16, 183-187.

Chabert, A.M.-H., 2017. Expression combinée des services écosystémiques en systèmes de production agricole conventionnels et innovants : étude des déterminants agroécologiques de gestion du sol, des intrants et du paysage, thèse de doctorat, spécialité Agrosystèmes, Ecosystèmes et Environnement, Institut National Polytechnique de Toulouse, $248 \mathrm{p}$.

Chichester, F.W., Richardson, C.W., 1992. Sediment and Nutrient Loss from Clay Soils as Affected by Tillage. Journal of Environmental Quality, 21, 587-590.

de Moraes Sa, J.C., Lal, R., 2009. Stratification ratio of soil organic matter pools as an indicator of carbon sequestration in a tillage chronosequence on a Brazilian Oxisol. Soil Tillage Research, 103, 46-56.

Dendooven, L., Gutiérrez-Oliva, V.F., Patiño-Zúñiga, L., RamírezVillanueva, D.A., Verhulst, N., Luna-Guido, M., Marsch, R., 
Montes-Molina, J., Gutiérrez-Miceli, F.A., Vásquez-Murrieta, S., Govaerts, B., 2012. Greenhouse gas emissions under conservation agriculture compared to traditional cultivation of maize in the central highlands of Mexico. Science of the Total Environment, 431, 237-244.

Derpsch, R., Friedrich, T., 2009. Development and Current Status of No-till Adoption in the World. In : Proceedings of the 18th Triennial Conference of the International Soil Tillage Research Organization (ISTRO), 15-19 juin, Izmir, Turkie.

Dimassi, B., Mary, B., Wylleman, R., Labreuche, J., Couture, D., Piraux, F., Cohan, J.P., 2014. Long-term effect of contrasted tillage and crop management on soilcarbon dynamics during 41 years. Agriculture, Ecosystems \& Environment, 188, 134-146.

Doran, J.W., 1980. Soil Microbial and Biochemical Changes Associated with Reduced Tillage. Soil Science Society of America Journal, 44, 765-771.

Drijber, R.A., Doran, J.W., Parkhurst, A.M., Lyon, D.J., 2000. Changes in soil microbial community structure with tillage under long-term wheat-fallow management. Soil Biology \& Biochemistry, 32, 1419-1430.

Ehler, L.E., 2006. Integrated pest management (IPM): definition, historical development and implementation, and the other IPM. Pest Management Science, 62, 787-789.

Farooq, M., Siddique, K.H.M., 2015a. Conservation Agriculture : Concepts, Brief History, and Impacts on Agricultural Systems. In : Conservation Agriculture (M. Farooq, K.H.M. Siddique, eds.), Springer International Publishing, Switzerland, pp. 3-17.

Farooq, M., Siddique, K.H.M., 2015b. Conservation Agriculture. Springer International Publishing, Switzerland, $665 \mathrm{p}$.

Faulkner, E.H., 1943. Plowman's folly, Michael Joseph Ltd, London, $175 \mathrm{p}$.

Fernández-Ugalde, O., Virto, I., Bescansa, P., Imaz, M.J., Enrique, A., Karlen, D.L., 2009. No-tillage improvement of soil physical quality in calcareous, degradation-prone, semiarid soils. Soil Tillage Research, 106, 29-35.

Fiedler, A.K., Landis, D.A., Wratten, S.D., 2008. Maximizing ecosystem services from conservation biological control: the role of habitat management. Biological Control, 45, 254-271.

FAO, 2008. Investing in Sustainable Agricultural Intensification. The Role of Conservation Agriculture. A framework for action. Food and Agriculture Organization of the United Nations, Rome, $24 \mathrm{p}$. 
Freixial, R., Carvalho, M., 2010. Aspectos prácticos fundamentales en la implantación de la agricultura de conservación/siembra directa en el sur de Portugal. In: Proceedings of the European Congress on Conservation Agriculture. Madrid, pp. 361-370.

Frey, S.D., Elliott, E.T., Paustian, K., 1999. Bacterial and fungal abundance and biomass in conventional and no-tillage agroecosystems along two climatic gradients. Soil Biology \& Biochemistry, 31, 573-585.

Friedrich, T., Derpsch, R., Kassam, A., 2012. Overview of the global spread of conservation agriculture. Field Actions Science Reports. The Journal of Field Actions, Special Issue 6, 0-7.

Gallai, N., Salles, J.-M., Settele, J., Vaissière, B.E., 2009. Economic valuation of the vulnerability of world agriculture confronted with pollinator decline. Ecological Economics, 68, 810-821.

IPCC, 2014. Climate Change 2014: Impacts, Adaptation, and Vulnerability. Contribution du $2^{\text {nd }}$ groupe travail au $5^{\text {ème }}$ rapport d'évaluation du GIEC (Intergovernmental Panel on Climate Change), United Kingdom and New York, 1132 p.

Gomiero, T., Pimentel, D., Paoletti, M.G., 2011. Environmental impact of different agricultural management practices: conventional vs. organic agriculture. Critical Reviews in Plant Science, $30,95-124$.

Grigera, M.S., Drijber, R.A., Wienhold, B.J., 2007. Redistribution of crop residues during row cultivation creates a biologically enhanced environment for soil microorganisms. Soil Tillage Research, 94, 550-554.

Guggenberger, G., Frey, S.D., Six, J., Paustian, K., Elliott, E.T., 1999. Bacterial and Fungal Cell-Wall Residues in Conventional and No-Tillage Agroecosystems. Soil Science Society of America Journal, 63, 1188-1198.

Gupta, R., Seth, A., 2007. A review of resource conserving technologies for sustainable management of the rice-wheat cropping systems of the Indo-Gangetic plains (IGP). Crop Protection, 26, 436-447.

Gurr, G.M., Wratten, S.D., Luna, J.M., 2003. Multi-function agricultural biodiversity: pest management and other benefits. Basic and Applied Ecology, 4, 107-116.

Hatten, T.D., Bosque-Pérez, N.A., Labonte, J.R., Guy, S.O., Eigenbrode, S.D., 2007. Effects of tillage on the activity density and biological diversity of Carabid beetles in psring and winter crops. Environmental Entomology, 36, 356-368. 
Hobbs, P., Sayre, K., Gupta, R., 2008. The role of conservation agriculture in sustainable agriculture. Philosophical Transactions of the Royal Society B, 363, 543-555.

Holland, J.M., 2004. The environmental consequences of adopting conservation tillage in Europe: reviewing the evidence. Agriculture, Ecosystems \& Environment, 103, 1-25.

Holland, J.M., Luff, M.L., 2000. The effects of agricultural practices on Carabidae in temperate agroecosystems. Integrated Pest Management Reviews, 5, 109-129.

Howarth, F.G., 2000. Non-target effects of biological control agents. In : Biological Control: Measures of Success (G. Gurr, S.D. Wratten, eds.), Kluwer Academic Publishers, Dordrecht, The Netherlands, pp. 369-403.

Ibekwe, A.M., Kennedy, A.C., Frohne, P.S., Papiernik, S.K., Yang, C.H., Crowley, D.E., 2002. Microbial diversity along a transect of agronomic zones. FEMS Microbiology Ecology, 39, 183-191.

Ishaq, S.L., 2017. Plant-microbial interactions in agriculture and the use of farming systems to improve diversity and productivity. AIMS Microbiology, 3, 335-353.

Jonsson, M., Wratten, S.D., Landis, D.A., Gurr, G.M., 2008. Recent advances in conservation biological control of arthropods by arthropods. Biological Control, 45, 172-175.

Kassam, A., Friedrich, T., Derpsch, R., Kienzle, J., 2015. Overview of the Worldwide Spread of Conservation Agriculture. Facts Reports, 8, 3-11.

Kassam, A., Friedrich, T., Derpsch, R., Lahmar, R., Mrabet, R., Basch, G., González-Sánchez, E.J., Serraj, R., 2012. Conservation agriculture in the dry Mediterranean climate. Field Crops Research, 132, 7-17.

Kell, D.B., 2012. Large-scale sequestration of atmospheric carbon via plant roots in natural and agricultural ecosystems: why and how. Philosophical Transactions of the Royal Society B, 367, 1589-1597.

Kesavan, P.C., Malarvannan, S., 2010. Green to evergreen revolution: ecological and evolutionary perspectives in pest management. Current Science, 99, 908-914.

Kladivko, E.J., 2001. Tillage systems and soil ecology. Soil Tillage Research, 61, 61-76.

Klein, A.-M., Vaissière, B.E., Cane, J.H., Steffan-Dewenter, I., Cunningham, S.A., Kremen, C., Tscharntke, T., 2007. Importance of pollinators in changing landscapes for world crops. 
Philosophical Transactions of the Royal Society B, 274, 303313.

Kosewska, A., Skalski, T., Nietupski, M., 2014. Effects of conventional and non-inversion tillage systems on the abundance and some life history traits of carabid beetles (Coleoptera: Carabidae) in winter triticale fields. European Journal of Entomology, 111, 669-676.

Kutcher, H.R., Johnston, A.M., Bailey, K.L., Malhi, S.S., 2011. Managing crop losses from plant diseases with foliar fungicides, rotation and tillage on a Black Chernozem in Saskatchewan, Canada. Field Crops Research, 124, 205-212.

Labreuche, J., Le Souder, C., Castilon, P., Ouvry, J.F., Real, B., Germon, J.C., De Tourdonnet, S., 2007. Evaluation des impacts environnementaux des Techniques Culturales Sans Labour en France, ADEME-ARVALIS Institut du végétal-INRA-APCAAREAS-ITB-CETIOM- IFVV, $400 \mathrm{p}$.

Lal, R., 2015a. Soil carbon sequestration and aggregation by cover cropping. Journal of Soil and Water Conservation, 70, 329-339.

Lal, R., 2015b. A system approach to conservation agriculture. Journal of Soil and Water Conservation, 70, 82A-88A.

Lal, R., 2015c. Cover cropping and the "4 per Thousand" proposal. Journal of Soil and Water Conservation, 70, 141A-141A.

Lal, R., 2011. Sequestering carbon in soils of agro-ecosystems. Food Policy, 36, S33-S39.

Lavorel, S., Sarthou, J.-P., 2008. Intérêts de la biodiversité pour les services rendus par les écosystèmes. In : Agriculture et Biodiversité - Valoriser Les Synergies (X. Le Roux, R. Barbault, J. Baudry, F. Burel, I. Doussan, E. Garnier, F. Herzog, S. Lavorel, R. Lifran, J. Roger-Estrade, J.-P. Sarthou, M. Trommetter, eds.), Expertise scientifique collective, rapport INRA, Paris, 738 p.

Leake, A.R., 2003. Integrated Pest Management for Conservation Agriculture. In : Conservation Agriculture: Environment, Farmers Experiences, Innovations, Socio-Economy, Policy (L. GarcíaTorres, J. Benites, A. Martínez-Vilela, A. Holgado-Cabrera, eds.), Springer Netherlands, Dordrecht, pp. 271-279.

Legrand, A., Gaucherel, C., Baudry, J., Meynard, J.M., 2011. Long-term effects of organic, conventional, and integrated crop systems on Carabids. Agronomy for Sustainable Development, 31, 515-524.

Lemanceau, P., Maron, P.A., Mazurier, S., Mougel, C., Pivato, B., Plassart, P., Ranjard, L., Revellin, C., Tardy, V., Wipf, D., 2015. Understanding and managing soil biodiversity: a major challenge 
in agroecology. Agronomy for Sustainable Development, 35, 67-81.

Marques, F., Basch, G., 2002. Comparação da viabilidade económica de quatro sistemas de mobilização do solo. In: I Congresso Nacional de Mobilização de Conservação Do Solo. Universidade de Évora, Evora, Portugal, pp. 283-298.

Marti, O.G., Olson, D.M., 2007. Effect of Tillage on Cotton Aphids (Homoptera: Aphididae), Pathogenic Fungi, and Predators in South Central Georgia Cotton Fields. Journal of Entomological Science, 42, 354-367.

McGonigle, T.P., Miller, M.H., Young, D., 1999. Mycorrhizae, crop growth, and crop phosphorus nutrition in maize-soybean rotations given various tillage treatments. Plant Soil, 210, 33-42.

MEA, 2005. Ecosystems and Human Well-being: Biodiversity Synthesis. Millennium Ecosystem Assessment, World Resources Institute, Washington, DC, $100 \mathrm{p}$.

Mulder, V.L., Lacoste, M., Richer-de-Forges, A.C., Martina, M.P., Arrouays, D., 2016. National versus global modelling the $3 \mathrm{D}$ distribution of soil organic carbon in mainland France. Geoderma, 263, 16-34.

Oerke, E.-C., 2006. Crop losses to pests. Journal of Agricultural Science, 144, 31-43.

Olson, K., Ebelhar, S.A., Lang, J.M., 2014. Long-Term Effects of Cover Crops on Crop Yields, Soil Organic Carbon Stocks and Sequestration. Open Journal of Soil Science, 4, 284-292.

Palm, C., Blanco-Canqui, H., DeClerck, F., Gatere, L., Grace, P., 2014. Conservation agriculture and ecosystem services: An overview. Agriculture, Ecosystems \& Environment, 187, 87-105.

Paustian, K., Lehmann, J., Ogle, S., Reay, D., Robertson, P., Smith, P., 2016. Climate-smart soils. Nature, 532, 49-57.

Pekrun, C., Kaul, H.P., Claupein, W., 2003. Soil tillage for sustainable nutrient management. In: Soil Tillage in Agroecosystems (A. El Titi, Ed.), CRC Press LLC, Boca Raton, pp. 83-113.

Pimentel, D., Burgess, M., 2014. Environmental and Economic Costs of the Application of Pesticides Primarily in the United States. In: Integrated Pest Management (D. Pimentel, R. Peshin, eds), Springer, pp. 47-71.

Pimentel, D., Wilson, C., McCullum, C., Huang, R., Dwen, P., Flack, J., Tran, Q., Saltman, T., Cliff, B., 1997. Economic and environmental benefits of biodiversity. Bioscience, 47, 747-757. Pisante, M., Stagnari, F., Acutis, M., Bindi, M., Brilli, L., Di Stefano, V., Carozzi, M., 2015. Conservation Agriculture and Climate 
Change. In: Conservation Agriculture (M. Farooq, K.H.M. Siddique, eds.), Springer International Publishing, Switzerland, pp. 579-620.

Pittelkow, C.M., Liang, X., Linquist, B. a., van Groenigen, K.J., Lee, J., Lundy, M.E., van Gestel, N., Six, J., Venterea, R.T., van Kessel, C., 2014. Productivity limits and potentials of the principles of conservation agriculture. Nature, 517, 365-368.

Plaza-Bonilla, D., Nolot, J.M., Passot, S., Raffaillac, D., Justes, E., 2016. Grain legume-based rotations managed under conventional tillage need cover crops to mitigate soil organic matter losses. Soil Tillage Research, 156, 33-43.

Powlson, D.S., Stirling, C.M., Jat, M.L., Gerard, B.G., Palm, C.A., Sanchez, P.A., Cassman, K.G., 2014. Limited potential of no-till agriculture for climate change mitigation. Nature Climate Change, 4, 678-683.

Powlson, D.S., Stirling, C.M., Thierfelder, C., White, R.P., Jat, M.L., 2016. Does conservation agriculture deliver climate change mitigation through soil carbon sequestration in tropical agroecosystems? Agriculture, Ecosystems \& Environment, 220, 164174.

Pretty, J., 2008. Agricultural sustainability: concepts, principles and evidence. Philosophical Transactions of the Royal Society B, 363, 447-465.

Pretty, J.N., Brett, C., Gee, D., Hine, R.E., Mason, C.F., Morison, J.I.L., Raven, H., Rayment, M.D., Van Der Bijl, G., 2000. An assessment of the total external costs of UK agriculture. Agricultural Systems, 65, 113-136.

Quinton, J.N., Catt, J.A., 2004. The effects of minimal tillage and contour cultivation on surface runoff, soil loss and crop yield in the long-term Woburn Erosion Reference Experiment on sandy soil at Woburn, England. Soil Use and Management, 20, 343-349.

Raymond, L., Sarthou, J.P., Plantegenest, M., Gauffre, B., Ladet, S., Vialatte, A., 2014. Immature hoverflies overwinter in cultivated fields and may significantly control aphid populations in autumn. Agriculture, Ecosystems \& Environment, 185, 99-105.

Rhoton, F.E., Shipitalo, M.J., Lindbo, D.L., 2002. Runoff and soil loss from midwestern and southeastern US silt loam soils as affected by tillage practice and soil organic matter content. Soil Tillage Research, 66, 1-11.

Roger-Estrade, J., Anger, C., Bertrand, M., Richard, G., 2010. Tillage and soil ecology: Partners for sustainable agriculture. Soil Tillage Research, 111, 33-40. 
Rutgers, M., van Wijnen, H.J., Schouten, A.J., Mulder, C., Kuiten, A.M.P., Brussaard, L., Breure, A.M., 2012. A method to assess ecosystem services developed from soil attributes with stakeholders and data of four arable farms. Science of the Total Environment, 415, 39-48.

Rypstra, A.L., Carter, P.E., Balfour, R.A., Marshall, S.D., 1999. Architectural features of agricultural habitats and their impact on the spider inhabitants. The Journal of Arachnology, 27, 371377.

Schulte, R.P.O., Creamer, R.E., Donnellan, T., Farrelly, N., Fealy, R., O'Donoghue, C., O'hUallachain, D., 2014. Functional land management: A framework for managing soil-based ecosystem services for the sustainable intensification of agriculture. Environmental Science \& Policy, 38, 45-58.

Selosse, M.A., Baudoin, E., Vandenkoornhuyse, P., 2004. Symbiotic microorganisms, a key for ecological success and protection of plants. Comptes Rendus Biologies, 327, 639-648.

Sharley, D.J., Hoffmann, A.A., Thomson, L.J., 2008. The effects of soil tillage on beneficial invertebrates within the vineyard. Agricultural and Forest Entomology, 10, 233-243.

Shearin, A.F., Reberg-Horton, S.C., Gallandt, E., 2007. Direct effects of tillage on the activity density of ground beetle (Coleoptera: Cababidae) weed seed predators. Environmental Entomology, 36, 1140-1146.

Shuler, R.E., Roulston, T.H., Farris, G.E., 2005. Farming practices influence wild pollinator populations on squash and pumpkin. Journal of Economical Entomology, 98, 790-795.

Singh, V.P., Barman, K.K., Singh, R., Sharma, A.R., 2015. Weed Management in Conservation Agriculture Systems. In: Conservation Agriculture (M. Farooq, K.H.M. Siddique, eds.), Springer International Publishing, Switzerland, pp. 39-77.

Six, J., Ogle, S.M., Breidt, J., Conant, R.T., Mosier, A.R., Paustian, K., 2004. The potential to mitigate global warming with no-tillage management is only realized when practised in the long term. Global Change Biology, 10, 155-160.

Soane, B.D., Ball, B.C., Arvidsson, J., Basch, G., Moreno, F., RogerEstrade, J., 2012. No-till in northern, western and south-western Europe: A review of problems and opportunities for crop production and the environment. Soil Tillage Research, 118, 66-87.

SoCo Project Team, 2009. Final report on the project Sustainable Agriculture and Soil Conservation (SoCo), European Commission, Scientific and Technical Research series, $172 \mathrm{p}$. 
Song, Z., Gao, H., Zhu, P., Peng, C., Deng, A., Zheng, C., Mannaf, M.A., Islam, M.N., Zhang, W., 2015. Organic amendments increase corn yield by enhancing soil resilience to climate change. The Crop Journal, 3, 110-117.

Spedding, T.A., Hamel, C., Mehuys, G.R., Madramootoo, C.A., 2004. Soil microbial dynamics in maize-growing soil under different tillage and residue management systems. Soil Biology \& Biochemistry, 36, 499-512.

Sturny, W.G., Chervet, A., 2015. Oberacker a fêté ses 20 ans : bilans et perspectives. TCS Magazine, 85, 6-25.

Tamburini, G., De Simone, S., Sigura, M., Boscutti, F., Marini, L., 2016. Conservation tillage mitigates the negative effect of landscape simplification on biological control. Journal of Applied Ecology, 53, 233-241.

Tebrügge, F., Böhrnsen, A., 1997. Crop yields and economic aspects of no-tillage compared to plough tillage: Results of long-term soil tillage field experiments in Germany. In: Experience with the Applicability of No-Tillage Crop Production in the WestEuropean Countries. Proceedings of the EC Workshop-IV. Langgöns, Germany, pp. 25-43.

Tebrügge, F., Düring, R.-A., 1999. Reducing tillage intensity - a review of results from a long-term study in Germany. Soil Tillage Research, 53, 15-28.

van Capelle, C., Schrader, S., Brunotte, J., 2012. Tillage-induced changes in the functional diversity of soil biota - A review with a focus on German data. European Journal of Soil Biology, 50, $165-181$.

Vieublé, L., 2015. Valoriser la composante biologique pour entretenir et améliorer la fertilité chimique et physique des sols. Formation continue AgroParis Tech MAROC, Paris.

Wardle, D.A., 1995. Impacts of disturbance on detritus food webs in agro-ecosystems of contrasting tillage and weed management practices. Advances in Ecological Research, 26, 105-185.

West, T.O., Post, W.M., 2002. Soil Organic Carbon Sequestration Rates by Tillage and Crop Rotation: A Global Data Analysis. Soil Science Society of America Journal, 66, 1930-1946.

Wilby, A., Thomas, M.B., 2002. Natural enemy diversity and pest control: patterns of pest emergence with agricultural intensification. Ecological Letters, 5, 353-360.

Williams, I.H., 1994. The dependences of crop production within the European Union on pollinsation by honey bees. Agricultural Zoology Reviews, 6, 229-257. 
Winqvist, C., Ahnstrom, J., Bengtsson, J., 2012. Effects of organic farming on biodiversity and ecosystem services: taking landscape complexity into account. Annals of the New York Academy of Science, 1249, 191-203.

Witmer, J.E., Hough-Goldstein, J.A., Pesek, J.D., 2003. GroundDwelling and Foliar Arthropods in Four Cropping Systems. Environmental Entomology, 32, 366-376.

Wright, A.L., Hons, F.M., Matocha, J.E., 2005. Tillage impacts on microbial biomass and soil carbon and nitrogen dynamics of corn and cotton rotations. Applied Soil Ecology, 29, 85-92. 

LA NOTION DE SERVICE ÉCOSYSTÉMIQUE PEUT-ELLE FONDER UN NOUVEAU RÉGIME DE PROTECTION? 



\section{Service écosystémique et financements publics}





\title{
La dépense publique en matière environnementale : l'exemple de la protection des sols
}

\author{
Liliane ICHER \\ Docteure en droit \\ post-doctorante Idex T2SEC
}

La présente contribution s'attachera à étudier comment l'Etat français et les collectivités territoriales ${ }^{1}$ peuvent protéger les sols par la dépense publique. A défaut d'encadrement réglementaire préservant ces ressources environnementales ${ }^{2}$, les personnes publiques peuvent engager des fonds publics à cette fin, passant d'une logique de contrainte à une logique d'incitation financière.

Etant donnée l'étendue de cette thématique et la conséquente impossibilité de la traiter entièrement et précisément dans le cadre de ce chapitre, il a fallu procéder à des choix méthodologiques pour délimiter le champ de l'étude. Une analyse sectorielle aurait pu être retenue. La dépense publique à destination de l'activité agricole aurait alors été privilégiée dans la mesure où « les écosystèmes agricoles sont de loin les plus grands écosystèmes aménagés du monde $»^{3}$ et que le lien entre cette activité et les services a été normativement consacré ${ }^{4}$. Cependant, des études très complètes portant sur les

1. La « dépense publique » renvoie aux dépenses engagées par les administrations publiques. La Sécurité sociale fait également partie des " administrations publiques» mais elle est exclue du champ de cette étude. En effet, il n'est pas prévu qu'elle engage des dépenses publiques pour protéger les sols (bien que la dégradation de l'environnement puisse entrainer des coûts sanitaires tel que le laisse transparaître l'article L. 110-1 II du $\mathrm{CE}$ ). Cette question dépasse le cadre de la présente contribution.

2. Cf. supra Hermon C. « La protection du sol en droit».

3. FAO, 2007, La situation mondiale de l'alimentation et de l'agriculture, p. 2.

4. L'article $2-8^{\circ}$ de la loi $n^{\circ} 2016-1087$ pour la reconquête de la biodiversité, de la nature et des paysages (RBNP) - aussi dite « biodiversité »- souligne la " complémentarité entre l'environnement, l'agriculture, L'aquaculture et la gestion durable des forêts, selon 
PSE dans ce secteur existent déjà ${ }^{5}$. Il a paru plus novateur de se pencher sur les outils financiers à disposition des personnes publiques pour améliorer la préservation des sols (notamment agricoles). Puis, toujours afin de préciser le champ d'étude, la démarche méthodologique suivante a été privilégiée : deux options de financement public relativement innovantes et diamétralement opposées ont été sélectionnées pour être comparées ${ }^{6}$. D'un côté, les administrations étatiques et locales peuvent convenir de « Paiements pour Services Environnementaux » (PSE) avec des personnes rémunérées pour préserver les sols ${ }^{7}$. De l'autre, elles pourraient créer un référentiel comptable vert intégrant la valeur desdits sols pour encourager leur protection. Les principales caractéristiques de ces deux modalités d'action doivent être dépeintes afin de comprendre en quoi elles sont radicalement différentes.

Avec les PSE, comme son nom l'indique, la préservation de la nature passe par la fourniture d'un service environnemental, qui requiert donc une intervention humaine. L'une des parties ${ }^{8}$ va œuvrer de manière à ce que les écosystèmes puissent fonctionner correctement. En échange, l'autre partie, parce qu'elle estime que cette forme de protection l'environnement lui est profitable - ici, l'Etat ou une

lequel les surfaces agricoles, aquacoles et forestières sont porteuses d'une biodiversité spécifique et variée et les activités agricoles, aquacoles et forestières peuvent être vecteurs d'interactions écosystémiques garantissant, d'une part, la préservation des continuités écologiques et, d'autre part, des services environnementaux qui utilisent les fonctions écologiques d'un écosystème pour restaurer, maintenir ou créer de la biodiversité ».

5. $C f$. notamment Langlais A. "Les paiements pour services environnementaux, une nouvelle forme d'équité environnementale pour les agriculteurs ? Réflexions juridiques ", Dr. rural, $\mathrm{n}^{\circ}$ 413, mai 2013, Etude $\mathrm{n}^{\circ}$ 7, du même auteur, L'agriculture et les paiements pour services environnementaux : quels questionnements juridique, Presses universitaires de Rennes, 2018, à paraître.

6. La fiscalité écologique ne sera pas ici examinée ; nous renvoyons à la lecture des nombreuses études consacrées à ce sujet, ainsi : Bin F. et Viessant C. «Précis de fiscalité de l'environnement », LexisNexis, à paraître, Caudal S. " La fiscalité de l'environnement », LGDJ, 2014, Caruana N. "La fiscalité environnementale. Entre impératifs fiscaux et objectifs environnementaux, une approche conceptuelle de la fiscalité environnementale », L'Harmattan, 2015,

7. Sur la liberté contractuelle des personnes publiques (et par conséquent, leur capacité à conclure ce type de convention), voir notamment Hastings-Marchadier A., « Les contrats de droit privé des personnes publiques et la liberté contractuelle », AJDA, 1998, pp. 683-693 et Pontier J.-M., « La liberté contractuelle des personnes publiques », AJDA 2013, pp. 837839.

8. Etant entendu que la catégorie des PSE ne recouvre pas que des contrats, $c f$. infra Poumarède M. « Services écosystémiques et contrat. Quelle obligation contractuelle environnementale ». 


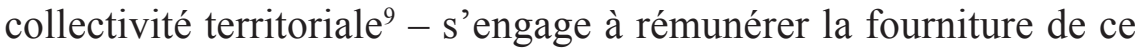
service. Le but est donc de rendre relativement moins attrayante l'exploitation d'une ressource naturelle par rapport aux perspectives financières qu'offre sa sauvegarde. En d'autres termes, il s'agit de passer d'une logique de pollueur-payeur, à celle de protecteurpercepteur ${ }^{10}$. Cette méthode s'inscrit dans la droite ligne de l'orientation actuellement privilégiée en droit de l'environnement, celle du consensualisme et de l'incitation pécuniaire ${ }^{11}$ plutôt que celle de l'interventionnisme public adoptée traditionnellement ${ }^{12}$. Plus encore, les PSE sont perçus comme des vecteurs de croissance économique parce qu'ils génèreraient des investissements et permettraient de créer des emplois ${ }^{13}$. Cet outil pourrait constituer un moyen temporaire de pallier une lacune juridique ${ }^{14}$, en l'espèce un régime protecteur des sols. Ces dispositions pourraient même être encore plus efficaces que les normes à portée générale puisqu'elles sont négociées et peuvent donc être ajustées aux besoins spécifiques d'un écosystème donné sur un territoire déterminé.

Autre voie d'action, plutôt que d'agir directement en engageant des dépenses, les personnes publiques pourraient œuvrer dans le sens de la protection des sols en adoptant une comptabilité qualifiée de « verte ». Quelques rappels sont nécessaires à la bonne compréhension de ce que signifie cette proposition. La comptabilité générale regroupe un ensemble de règles, appelé « référentiel », qui est supposé donner, selon la terminologie consacrée, une « image fidèle $\|{ }^{15}$ de la situation patrimoniale et financière d'une personne morale privée ${ }^{16}$. Cependant, en droit public, les règles comptables

9. Langlais A, « Les paiements pour services environnementaux, une nouvelle forme d'équité environnementale pour les agriculteurs ? Réflexions juridiques ", op. cit., §12. L'article L. 312-3 du C. env. précise, depuis la loi « biodiversité », que les contrats en question peuvent être conclus avec une personne privée mais aussi l'Etat, une collectivité territoriale ou même un établissement public.

10. Sutterlin O., « Le principe pollueur-payeur », J.-Cl. Env. et DD, fasc. 2024, §11.

11. Conseil économique pour le développement durable, Bureau D. (dir.), « les "PSE": des rémunérations pour les services environnementaux », 2010, $\mathrm{n}^{\circ} 17$.

12. Langlais A., op. cit., $\S 12$.

13. De Perthuis C. et Jouvet P.-A, op. cit., p. 126.

14. Sutterlin O., op. cit., $§ 11$.

15. La comptabilité ne saurait donner une image exacte de l'entité concernée mais doit permettre à ses lecteurs de se faire une idée claire de sa situation patrimoniale et financière. 16. La comptabilité générale privée - à destination des entreprises - a largement influencé les référentiels comptables appliqués aux personnes publiques, voir notamment Collet M., Finances publiques, LGDJ, 2016, p. 108. L'exigence d'une image fidèle pour les comptes des personnes publiques a dorénavant valeur constitutionnelle suite à la révision de 2008, 
sont plus complexes parce qu'elles doivent saisir les spécificités la décision publique ${ }^{17}$. Aussi, la « comptabilité nationale » retrace les engagements juridiques des personnes publiques mais elle va au-delà en recensant des données statistiques sur l'état de l'économie française ${ }^{18}$. Il s'agit donc d' " une technique d'enregistrement et d'évaluation des événements affectant la situation financière d'une entité bien définie et de présentation de ces informations dans des états de synthèse [mais elle permet également] de calculer des indicateurs globaux ${ }^{19}{ }^{19}$, parmi lesquels le Produit Intérieur Brut (PIB). Cette valeur traduit la production annuelle de richesses sur un territoire qui émane à la fois du secteur public, des entreprises et des ménages. La comptabilité nationale est alors susceptible de fournir une base de décision sur les mesures politiques à prendre. Or, la comptabilité environnementale consiste à intégrer dans les comptes nationaux la valeur des éléments naturels, notamment celle des sols. Le but est de « verdir » le PIB $^{20}$ pour orienter les choix politiques - dont les dépenses publiques - dans un sens plus écologique. La complexité technique de l'entreprise est régulièrement utilisée comme argument pour s'opposer à l'avancement de ce projet. L'élaboration d'un référentiel de comptabilité verte serait intellectuellement concevable mais trop peu maniable pour devenir un outil opérationnel de gestion et un référentiel contraignant.

S'il ne fallait en choisir qu'un, quel serait l'outil de dépense publique le plus utile à la protection des sols ? A priori, les PSE paraissent constituer une solution simple qui permet à l'administration d'entrer en action immédiatement et de cibler précisément les sols. Pourtant,

Kott S. (dir.), Droit et comptabilité, La spécificité des comptes publics, Economica, 2017, p. 4. Elle est inscrite à l'article 47-2 de la Constitution.

17. Milot J.-P., «L'information financière sur les finances publiques : périmètre, utilité et portée », RFFP, n 122 , avril 2013, p. 137.

18. Contrairement à la comptabilité nationale qui mobilise la méthode des «droits constatés » pour l'ensemble des Administrations Publiques (APU) et qui intègre des données statistiques sur l'ensemble de l'économie française, le référentiel de « comptabilité publique " apparaît moins adapté au verdissement à trois égards : d'abord, il ne prend pas en considération l'ensemble de l'économie ; ensuite, il diffère selon la catégorie d'APU concernée ; il emploie la méthode des « encaissements/décaissements ».

19. Milot J.-P., op. cit.

20. Cf. Angel M., La nature a-t-elle un prix ? Critique de l'évaluation des biens environnementaux, Les presses Ecole des Mines de Paris, 1998, p. 3. Bien que l'auteur n'adhère pas aux méthodes actuelles de comptabilisation verte, il relève que « le PIB [est inadapté] à la gestion des biens environnementaux : leur détérioration ne se traduisant par aucun échange monétaire, elle n'y est simplement pas comptabilisée (...) comment y remédier ? L'idée proposée par les économistes est de doter l'environnement d'une valeur monétaire ». 
lorsque les PSE sont conclus par des personnes publiques, ils créent de nombreuses difficultés juridiques qui les rendent finalement peu attrayants, voire peu praticables (I). Au contraire, « la construction des outils comptables dont sont dotées les administrations publiques semble, à première vue, aussi technique que rébarbative. Elle soulève pourtant des enjeux considérables, tant sur le plan pratique que théorique $\gg^{21}$. Bien que les difficultés d'élaboration d'un référentiel et de mise en œuvre soient réelles, le potentiel de la comptabilité verte semble sous-estimé. Les opportunités qu'offrirait cette solution, justifient de fournir un effort intellectuel de long terme dont les retombées en termes de dépenses publiques ne seront qu'indirectes (II).

\section{LES PSE : LES LIMITES D'UNE DÉPENSE PUBLIQUE ACTUELLE}

La sauvegarde des sols par la conclusion de PSE engendre des coûts pour les personnes publiques. Or, ces engagements financiers se révèlent, au mieux peu efficaces en termes de préservation des sols (A), et au pire, risqués en raison des incertitudes juridiques qu'ils portent lorsqu'ils sont pris par des personnes publiques (B). En effet, des problématiques spécifiques apparaissent lorsque le preneur de service environnemental est une personne publique parce que celleci est présumée perturber l'équilibre du marché lorsqu'elle intervient dans l'économie. Son action est alors étroitement encadrée par le droit pour éviter qu'il y ait distorsion de concurrence. En revanche, si un PSE au contenu strictement identique est signé par une personne privée, sa décision est censée relever du fonctionnement normal de l'économie. Alors, cette opération ne sera pas entravée par des contraintes juridiques ${ }^{22}$.

\section{A. L'efFICACITÉ DISCUTABLE DES DENIERS PUBLICS ENGAGÉS DANS LES PSE}

Les PSE ne peuvent constituer qu'une façon marginale de protéger l'environnement, et en l'occurrence, les sols. Si les limites qui vont être développées s'appliquent à tous ces dispositifs, ils posent une

21. Collet M., op.cit., p. 491.

22. $C f$. infra Poumarède M., "Services écosystémiques et contrat. Quelle obligation contractuelle environnementale ». 
question spécifique quand ils sont adoptés par une administration, celle de la « bonne utilisation des deniers publics $»^{23}$.

La nature consensuelle des PSE qui est présentée comme étant l'avantage principal de ce mode de sauvegarde, en constitue aussi la faiblesse tel que 1'a d'ores et déjà souligné la doctrine : sans consentement de l'éventuel fournisseur de service environnemental, pas de préservation. Afin d'obtenir cet accord, les contraintes - parmi lesquelles la durée de l'engagement ${ }^{24}$ - peuvent être assouplies réduisant d'autant la protection effective des sols. D'ailleurs, ainsi que le souligne la doctrine, la majorité des PSE existants ne contient que des obligations de moyen et non de résultat écologique. Alors, le fournisseur de service environnemental ne s'oblige qu'à faire ou ne pas faire, peu importe l'effet de la convention sur la nature, ce qui abaisse le niveau d'exigence qu'il a à supporter et facilite par là même l'obtention de son consentement ${ }^{25}$. Notons que la terminologie usitée - et que l'on continuera d'employer dans cette contribution par souci de cohérence avec le droit positif - n'est donc pas adaptée : le paiement ne vient pas rémunérer la fourniture d'un service environnemental mais une pratique ; cette remarque vaut également pour les mesures agro-environnementales et climatiques (MAEC) prévues par le deuxième pilier de la $\mathrm{PAC}^{26}$.

23. Plus qu'une seule considération politique, il s'agit bien d'une question juridique en ce que le « bon usage des deniers publics » fait partie du droit positif. Quoiqu'imprécisément définie, cette expression est désormais une exigence constitutionnelle (Cons. Constit., 26 juin 2003, n 2003-473 DC, AJDA, 2003, p. 1404, note E. Fatôme) et un élément d'appréciation employé par le juge administratif. Mais, en dehors même de la situation contentieuse, le bon usage des deniers publics sert de guide aux décideurs publics lorsqu'ils effectuent le bilan coûts/avantages d'une dépense à venir, Boiteux D., « Le bon usage des deniers publics », $R D P, \mathrm{n}^{\circ}$ 5, sep. 2011, pp. 1099-1135.

24. Or, pour être environnementalement efficaces, les PSE doivent contraindre sur une période suffisamment longue pour se calquer sur le temps biologique, Etrillard C., «Paiements pour services environnementaux : nouveaux instruments de politique publique environnementale », Développement durable et territoires, vol. 7 n 1 , p. 6.

25. Par souci d'honnêteté, ajoutons que cette limite s'explique aussi par d'autres facteurs. Etant donné la complexité du fonctionnement des sols et les incertitudes scientifiques qui demeurent, rédiger un contrat fixant des obligations de résultat ne saurait être aisé. Rémunérer, mais aussi contrôler, des actions humaines s'avère beaucoup plus simple, Doussan I. et Martin G.-J., " Les PSE à la lumière de la théorie générale des contrats », in L'agriculture et les paiements pour services environnementaux : quels questionnements juridiques?, Langlais A. (dir.), 2017, à paraître, pp. 1-13.

26. Doussan I. et Martin G.-J., " Les PSE à la lumière de la théorie générale des contrats », op. cit., p. 5. 
Toujours pour emporter l'adhésion du producteur de service environnemental, une rémunération est offerte. Et, là encore, les PSE portent en eux les raisons de leurs propres limites : si la motivation desdits fournisseurs est avant tout financière, ceuxci sont susceptibles de chercher à profiter de la manne que cette nouvelle possibilité offre. Dans un premier temps, les PSE sont susceptibles de provoquer un effet d'aubaine : les bénéficiaires pourraient se porter volontaires simplement parce qu'ils adoptent déjà les comportements encouragés sans être rétribués pour. Cette situation est d'autant plus vraisemblable que les dispositifs ne posent pas de résultats environnementaux précis et exigeants ${ }^{27}$. Alors, la personne publique qui conclurait un PSE, engagerait une dépense inutile dans la mesure où elle paierait pour maintenir un statu quo, pour éviter la menace d'un changement de pratiques néfaste aux sols. Ensuite, une fois la prestation terminée, le producteur de service environnemental pourrait aussi revoir à la hausse ses prétentions financières pour accepter de poursuivre ses efforts (et ce, même si lesdits efforts n'en n'étaient pas réellement ab initio). Ce piège est au moins partiellement déjoué en matière agricole. En effet, dans le cadre de la PAC pour bénéficier des aides agroenvironnementales du deuxième pilier, les professionnels doivent respecter des normes réglementaires pour pouvoir ensuite prétendre à une rémunération. Ces « normes plancher [...] constituent un garde-fou concernant le contenu environnemental des PSE, lesquels ne peuvent ainsi être éligibles au financement qu'audelà de la réalisation de ce minimum $\gg^{28}$. Les frais supplémentaires engagés par les agriculteurs sont alors compensés par la personne publique.

Au-delà de la question des « retours environnementaux sur investissement » que génèrerait ce choix, une généralisation des PSE pourrait s'avérer plus coûteuse pour les personnes publiques que ce qui est stipulé.

27. Cette difficulté avait déjà été soulevée dans le cadre d'un autre marché environnemental. Ducret P. et Scolan M., "Le prix du carbone : la valeur d'une expérience », Vraiment durable, vol. $4, \mathrm{n}^{\circ} 2,2013$, pp. 47-53.

28. Langlais A., op. cit., $\S 15$. 
B. Les fRAgilités JuRidiQues des PSE Conclus Par les Personnes PUBLIQUES

Ala question de l'efficacité des PSE, s'ajoutent des risques juridiques importants. Ceux-ci tiennent au fait que cette opération n'est pas évidente à qualifier en droit - national (1) et européen (2) - quand la partie rémunératrice est une personne publique et quand le contrat ne relève pas des aides de la PAC.

\section{1) Les PSE et la commande publique en droit interne}

En droit interne, l'identification du PSE pose un premier ensemble de problèmes juridiques. Pour la personne publique, conclure un PSE ne lui permet pas à proprement parler de «satisfaire un besoin mais [d'] orienter l'activité d'un opérateur privé conformément à l'intérêt général $»^{29}$. Si le fournisseur de service environnemental sollicite la personne publique pour percevoir une somme lui permettant de poursuivre son activité, le PSE correspond à ce que l'on qualifie de « convention d'objectifs et de moyens » telle que définie dans l'arrêt du Conseil d'Etat «Commune d'Aix-en-Provence $»^{30}$, reprise par la suite dans la circulaire du 18 janvier $2010^{31}$. Alors, la rémunération octroyée s'apparente à une subvention ${ }^{32}$.

En revanche, si la personne publique est à l'initiative - ce qui paraît plus que plausible dans l'hypothèse de l'impulsion d'une politique publique de protection des sols - la rémunération du PSE n'est plus considérée comme une subvention mais elle est assimilée à un prix ${ }^{33}$.

29. Nicinski S., «Besoins », Droit des marchés publics \& Contrats publics spéciaux, Le Moniteur, coll. Moniteur Références, T. 1, II.410, mise à jour n ${ }^{\circ} 71$, juill. 2012, p. 4.

30. CE sect., 6 avril 2007, Commune d'Aix-en-Provence, JCP A 2007, ${ }^{\circ} 2111$, note Karpenschif M.

31. Circulaire du 18 janvier 2010 relative aux relations entre les pouvoirs publics et les associations : conventions d'objectifs et simplification des démarches relatives aux procédures d'agrément. Les conventions d'objectifs sont généralement conclues avec des associations. Cependant, la circulaire de 2010 rappelle à l'article 4 que l'attribution de subventions ne se limite pas à elles.

32. Si la subvention est égale ou supérieure à 23000 euros par an, elle doit être consignée dans une convention « définissant l'objet, le montant, les modalités de versement et les conditions d'utilisation », article 10, loi n ${ }^{\circ} 2000-321$ du 12 avr. 2000 relative aux droits des citoyens dans leurs relations avec les administrations. Ceci ne pose pas de problème dans la mesure où la conclusion d'un PSE nécessite la rédaction d'un contrat qui contient ces éléments.

33. Article 4 b, circulaire du 18 janvier 2010, op. cit.. Dans la lignée de la jurisprudence Commune d'Aix-en-Provence, la circulaire donne la prévalence à l'initiative du projet 
Dès lors, si la personne publique verse une somme à une personne qui lui délivre une prestation, le contrat intègre le périmètre des marchés publics ${ }^{34}$. Or, ceci emporte des conséquences lourdes. Les procédures inscrites dans le Code des marchés publics (CMP) doivent être respectées si le contrat entraîne une rémunération du cocontractant supérieure ou égale aux seuils fixés par décret ${ }^{35}$. Lesdites procédures requièrent en premier lieu la définition précise des besoins pour que la comparaison des projets des concurrents soit effective. Une description trop floue des attentes pesant sur le futur cocontractant de la personne publique peut engager la responsabilité de 1'administration vis-à-vis des candidats éconduits ${ }^{36}$. Or, la complexité du fonctionnement des écosystèmes rend délicate la rédaction d'un appel à la concurrence. Une fois ce stade franchi, les procédures de passation des marchés publics comptent d'autres étapes, assorties de délais, qui doivent être respectées pour ne pas risquer le prononcé de sanctions pécuniaires ou l'annulation de la décision de passer la convention. De plus, si l'on s'inscrit dans le cadre du marché public, le juge administratif peut se fonder sur le bon usage des deniers publics, principe énoncé dès l'article $1^{\mathrm{er}} \mathrm{du}$ CMP, pour régler le litige qui lui est soumis. La question de l'efficacité des PSE pour protéger les sols qui a été évoquée précédemment, pourrait dépasser le cadre d'une simple question politique pour devenir un enjeu juridique. Ajoutons que le non-respect de ces règles de passation est susceptible d'entrainer l'ouverture de poursuites pénales à l'encontre des personnes physiques et qu'elles conduisent potentiellement à des peines d'emprisonnement et des amendes des poursuites pourraient même être ouvertes à l'encontre de personnes

comme indicateur de la présence, ou non, d'une convention d'objectifs, Nicinski S., Le droit public des affaires, LGDJ, 2016, p. 571.

34. Cf. infra Hoepffner H. « La commande publique de compensation environnementale : un impensé de la loi Biodiversité ». La distinction entre les marchés publics et les DSP peut brièvement être décrite ainsi : dans le premier contrat, l'administration satisfait un de ses besoins et rémunère l'autre partie en une fois ; dans le second, elle transfère temporairement l'exécution d'un service public à un tiers qui sera payé régulièrement. Ainsi, il semblerait que le PSE puisse tomber dans l'une ou l'autre de ces catégories; pour déterminer la nature de la convention, il faut se référer à son contenu.

35. Ces seuils sont multiples et variables. D'abord, ils dépendent du type de marché public en question : ils sont donc susceptibles de changer selon que le contenu du PSE le classe parmi les marchés de biens, de services ou de travaux. Ensuite, ils seraient différents si le PSE était rangé dans la catégorie des DSP. Finalement, les seuils fluctuent régulièrement au fil du temps du temps. $C f$. infra Hoepffner $\mathrm{H}$. « La commande publique de compensation environnementale : un impensé de la loi Biodiversité.

36. Nicinski S., Le droit public des affaires, op. cit., p. 591. 
physiques pour délit d'octroi d'avantage injustifié37 ou corruption et trafic d'influence ${ }^{38}$. En conclusion, la qualification potentielle des PSE en marchés publics serait chronophage et potentiellement onéreux.

\section{2) Les PSE en droit de l'Union}

En droit de l'union, les PSE pourraient être qualifiés d'aides d'Etat ou de Services d'Intérêt Economique Général (SIEG).

Les PSE pourraient constituer des aides d'Etat, par définition, illégales ${ }^{39}$. En effet, en vertu de l'article $107 \S 1$ du Traité sur le Fonctionnement de l'Union Européenne (TFUE), « sauf dérogations prévues par les traités, sont incompatibles avec le marché intérieur, dans la mesure où elles affectent les échanges entre États membres, les aides accordées par les États ou au moyen de ressources d'État sous quelque forme que ce soit qui faussent ou qui menacent de fausser la concurrence en favorisant certaines entreprises ou certaines productions ». En d'autres termes, il y a aide d'Etat parce qu' « en recevant, alors même qu'il ne satisfait aucun besoin marchand, un financement public visant uniquement à le maintenir ou l'orienter vers des pratiques pro-environnementales, [le fournisseur de service environnemental] est [...] perçu, au regard du droit de l'Union européenne, comme bénéficiant d'un soutien financier auquel aucun acteur économique n'aurait consenti au regard des seules considérations guidant le fonctionnement normal du marché $»^{40}$. Toutefois, ce principe connait à la fois des dérogations textuelles qui seraient théoriquement applicables à tous les PSE et des exceptions propres.

37. Aussi appelé « délit de favoritisme », article 432-14 C. pén.

38. Article 432-11 C. pén.

39. Ces soutiens financiers illégaux ne se limitent évidemment pas aux PSE, ils peuvent prendre diverses formes. Ainsi, ils incluent les « subventions, exonérations d'impôts et de taxes, exonérations de taxes parafiscales, bonifications de taux d'intérêt, garanties de prêts consenties dans des conditions particulièrement favorables, cessions de bâtiments ou de terrain à titre gratuit ou à des conditions particulièrement favorables, fournitures de biens ou de services à des conditions préférentielles, couvertures de pertes d'exploitation ou toute autre mesure d'effet équivalent » (réponse à une question écrite, JOCE C 125, 17 août 1963) et cette liste n'est pas exhaustive, Vade-mecum des aides d'Etat, édition 2016, fiche 1, p. 21. 40. Caylet S., "Contrats publics et paiements pour services environnementaux », in L'agriculture et les paiements pour services environnementaux : quels questionnements juridiques?, Langlais A. op. cit. 
La première catégorie de dérogations générales est à trouver à l'article $107 \S 2$ du TFUE ${ }^{41}$ : certains soutiens financiers publics ne sont pas considérés comme des aides d'Etat et sont par là même compatibles avec le marché intérieur. Cependant, les mesures financières destinées à protéger l'environnement - et plus précisément, les sols - ne figurent pas dans cette liste ${ }^{42}$. Ensuite, l'article énonce au troisième paragraphe que, sur notification des Etats membres, des aides peuvent être considérées comme compatibles avec le marché intérieur, et de ce fait, être autorisées par la Commission. Certaines catégories définies seraient assez vastes pour y inclure une politique de protection de l'environnement - et notamment des sols - dont «b) les aides destinées à promouvoir la réalisation d'un projet important d'intérêt européen commun » (PIIEC) telle que la stratégie Europe $2020^{43}$ 《 c) les aides destinées à faciliter le développement de certaines activités [...], quand elles n'altèrent pas les conditions des échanges dans une mesure contraire à l'intérêt commun » et «e) les autres catégories d'aides déterminées par décision du Conseil sur proposition de la Commission ». Toutefois, ces exceptions au principe d'incompatibilité sont interprétées de façon restrictive ${ }^{44}$ donc l'autorisation des PSE sur la base de la disposition $107 \S 3$ du TFUE paraît très incertaine. Finalement, le Règlement Général d'Exemptions par Catégorie (RGEC) du 17 juin $2014^{45}$ permet de dispenser certaines aides environnementales de la notification des Etats membres prévue à l'article $107 \S 3$. Il pourrait s'avérer utile

41. Pour être exhaustif, il convient d'ajouter que si toutes les conditions de l'article 107 $\S 1$ du TFUE n'étaient pas remplies, la somme versée ne serait plus considérée comme une aide d'Etat. Toutefois, les cas de figure qui correspondent à cette situation paraissent très théoriques et ne seront pas développées plus avant. En effet, il faudrait que le montant ne soit pas accordé par une personne publique ou qu'il ne soit pas distribué à une entreprise ou qu'il soit, au contraire, octroyé à toutes les entreprises pour ne pas créer de distorsion de concurrence.

42. Seuls les cas suivants sont envisagés : « a) les aides à caractère social octroyées aux consommateurs individuels [l'aide est octroyée à une entreprise intermédiaire mais elle ne doit pas en bénéficier, l'avantage doit être répercuté sur certaines catégories de consommateurs finaux], b) les aides destinées à remédier aux dommages causés par les calamités naturelles ou par d'autres événements extraordinaires, c) les aides octroyées à l'économie de certaines régions de la république fédérale d'Allemagne affectées par la division de l'Allemagne $»$.

43. En 2011, l’UE a lancé la « Stratégie Europe 2020 » qui comprend une liste d'actions à mener pour encourager la croissance au sein de l'Union. Elle comprend notamment des actions environnementales. Le projet est présenté dans le document disponible en ligne : http://www.construireleurope.org/pprod/wp-content/uploads/2014/02/FT-EU2020.pdf 44. Vade-mecum des aides d'Etat, édition 2016, fiche 3, p. 67.

45. Règlement $\mathrm{n}^{\circ} 651 / 2014$ du 17 juin 2014 déclarant certaines catégories d'aides compatibles avec le marché intérieur en application des articles 107 et 108 du traité. 
mais uniquement dans un type très précis de PSE relatifs aux sols : il autorise les Etats et leurs entités publiques à octroyer des fonds sans l'aval des institutions européennes jusqu'à 20 millions d'euros par entreprise et par projet pour les investissements en faveur de l'assainissement d'un site contaminé «sur lequel a été confirmée la présence de substances dangereuses découlant de l'activité humaine, dans des concentrations telles qu'elles présentent un risque important pour la santé humaine ou pour l'environnement compte tenu de l'utilisation effective des terrains et de leur utilisation future autorisée ${ }^{46}$. Si un PSE correspond à cette disposition du règlement, il faut en plus que la rémunération soit transparente, incitative et publiée ${ }^{47}$.

Contrairement aux dérogations générales dont la mobilisation semble presque impossible en pratique pour les PSE protégeant les sols, l'ensemble des exceptions spécifiques au secteur agricole est régulièrement appliqué.

Aux termes de l'article 42 TFUE, les règles prévues aux articles 107 et suivants du TFUE, ne sont pas automatiquement applicables aux aides PAC; elles ne le sont que « que dans la mesure déterminée par le Parlement européen et le Conseil ». Ainsi, les aides d'Etat en matière agricole sont le plus souvent régies par des normes spécifiques ${ }^{48}$ comprenant un règlement de minimis ${ }^{49}$, des lignes directrices ${ }^{50}$ et un régime d'exemption agricoles ${ }^{51}$. Il ressort de la combinaison de ces

\footnotetext{
46. Article 2 du règlement $n^{\circ}$ 651/2014, op. cit., alors que le seuil de minimis de droit commun est de $200000 €$ sur une période de trois exercices fiscaux, art. 3.2 règlement $\mathrm{n}^{\circ} 1407 / 2013$ du 18 décembre 2013 relatif à l'application des articles 107 et 108 du traité sur le fonctionnement de l'Union européenne aux aides de minimis.

47. Art. 3 et suivants du règlement $\mathrm{n}^{\circ} 651 / 2014$, op. cit.

48. Si une aide agricole non prévue dans les textes spécifiques à cette activité contient des dispositions environnementales, ce sont les règles relatives aux aides d'Etat environnementales qui s'appliquent, Vade-mecum des aides d'Etat, édition 2016, fiche 13, p. 211.

49. Règlement $n^{\circ} 1408 / 2013$ du 18 décembre 2013 relatif à l'application des articles 107 et 108 du traité sur le fonctionnement de l'Union européenne aux aides de minimis dans le secteur de l'agriculture.

50. Lignes directrices de l'Union européenne concernant les aides d'État dans les secteurs agricole et forestier et dans les zones rurales 2014-2020, 2014/C 204/01.

51. Règlement $\mathrm{n}^{\circ} 702 / 2014$ de la Commission du 25 juin 2014 déclarant certaines catégories d'aides, dans les secteurs agricole et forestier et dans les zones rurales, compatibles avec le marché intérieur, en application des articles 107 et 108 du traité sur le fonctionnement de l'Union européenne.
} 
textes que les aides agro-environnementales du deuxième pilier ${ }^{52}$ peuvent concourir à la protection des sols. Ainsi que le souligne le règlement de juin 2014, les investissements sont susceptibles d'être compensés par des aides publiques s'ils poursuivent l'un des objectifs énoncés à l'article 3 même texte ${ }^{53}$, dont « la mise en œuvre des objectifs agroenvironnementaux et climatiques ». C'est ainsi que certaines aides versées au titre des MAEC peuvent bénéficier aux sols ${ }^{54}$.

En plus des exceptions textuelles (générales ou spécifiques), les versements financiers d'une personne publique peuvent être autorisés s'ils sont reconnus par les juges européens comme étant des Services d'Intérêt Economique Général (SIEG). Bien que cette qualification ne permette pas d'échapper au droit de la concurrence, elle autorise la juste compensation des coûts générés par la satisfaction d'obligation de service public. En effet, depuis le fameux arrêt «Altmark » ${ }^{55}$, un tel soutien financier public échappe au régime des aides d'Etat si quatre conditions sont remplies : "l'entreprise bénéficiaire doit effectivement être chargée de l'exécution d'obligations de service public, et ces obligations doivent être clairement définies »; "les paramètres sur la base desquels est calculée la compensation doivent être préalablement établis, de façon objective et transparente, afin d'éviter qu'elle comporte un avantage économique susceptible de favoriser l'entreprise bénéficiaire par rapport à des entreprises concurrentes »; " la compensation ne saurait dépasser ce qui est nécessaire pour couvrir tout ou partie des coûts occasionnés par l'exécution des obligations de service public, en tenant compte des recettes y relatives ainsi que d'un bénéfice raisonnable »; " lorsque le choix de l'entreprise [...] n'est pas effectué dans le cadre d'une procédure de marché public [...] le niveau de la compensation nécessaire doit être déterminé sur la base d'une analyse des coûts qu'une entreprise moyenne bien gérée et adéquatement équipée ${ }^{56}$. Ces critères appellent deux remarques. D'une part, le « bénéfice raisonnable » constitue une incitation financière minimale réduisant

52. Voir spéc. les points 207 et suivants des lignes directrices pour la période actuelle.

53. Article 14.3.d du règlement $\mathrm{n}^{\circ} 702 / 2014$, op. cit.

54. Cf. supra Hermon C. « La protection du sol en droit».

55. CJCE, 24 juill. 2003, aff. C-280/00, Dr. adm. 2003, n 186.

56. Au regard de cette définition, les MAEC semblent proches des SIEG puisqu'il s'agit de compenser les surcoûts supportés par les agriculteurs qui vont au-delà de leurs obligations normatives pour adopter des pratiques favorables à l'environnement. Mais encore faudraitil que l'activité menée puisse être dite relever du service public. 
par là même l'attractivité des PSE conçu comme SIEG. D'autre part, plus fondamentalement, il n'est pas sûr que la sauvegarde des sols puisse être actuellement interprétée comme relevant du service public, dès lors les PSE ne pourraient pas être conçus comme des SIEG. La liste de ces services n'est pas exhaustive mais elle comprend traditionnellement les postes, transports, télécommunications et énergie. Elle est appelée à s'étendre mais ce mouvement est lent et il faut rester prudent. Pour l'heure, « il est impossible de déterminer $a$ priori si la protection de l'environnement est susceptible de participer de l'intérêt économique général au sens de la notion de SIEG. On ne peut pas non plus déterminer les activités environnementales particulières qui peuvent en relever $\rangle^{57}$.

En conclusion, signer des PSE pourrait s'avérer dangereux pour les personnes publiques, en plus d'être relativement coûteux et peu efficace. Il s'agit d'un moyen de protéger l'environnement conçu pour le secteur privé et qui peut être intéressant dans ce cadre ${ }^{58}$, mais ces contrats ne sauraient constituer un outil pertinent pour une politique d'envergure mise en œuvre par les personnes publiques. Au contraire, la comptabilité verte sur la dépense publique pourrait avoir un impact plus fondamental sur la protection des sols.

\section{LA COMPTABILITÉ ENVIRONNEMENTALE : LE POTENTIEL D'UNE DÉPENSE PUBLIQUE ÉVENTUELLE}

L'intégration des éléments naturels à la comptabilité nationale pourrait constituer une voie médiane prometteuse, une solution qui ne s'apparente ni à une intervention unilatérale publique classique, ni à un alignement sur les méthodes consensuelles et incitatives créées pour le secteur privé.

L'instauration d'un référentiel comptable vert relève d'une méthode de protection de l'environnement globalisante en ce qu'elle intègre la totalité des éléments naturels, les sols et leurs services compris. Malgré la persistance de certaines résistances politiques, le potentiel de cette orientation a bien été identifié, à la fois par ses premiers soutiens universitaires ainsi que par les institutions internationales 
et européennes qui élaborent désormais des référentiels verts (A). Ce type de système comptable vise à fondamentalement repenser les finances publiques, et particulièrement à réorienter les décisions de dépenses dans une direction plus écologique. D’ailleurs, des exemples concrets de verdissement des finances publiques françaises laissent penser que la dynamique est enclenchée, que la valorisation des éléments naturels pourrait bel et bien constituer un développement futur possible de la comptabilité utilisée par les personnes publiques (B).

\section{A. Les laCunes de LA COMPTABilité nATIONALE TRADITIONNELLE ET LES PRÉMISSES DE RÉFÉRENTIELS VERTS À DISPOSITION DES PERSONNES PUBLIQUES}

Donner une valeur monétaire à des éléments qui ne font pas l'objet de commerce peut sembler quelque peu artificiel. Pourtant, une convention (au sens de « standard accepté par une communauté $»^{59}$ ), si abstraite, détachée de la réalité soit-elle, permet de s'accorder sur ce qui deviendra des points de repère. Certes, l'information produite est imparfaite parce qu'elle ne s'adosse pas à des transactions observables ${ }^{60}$ et repose sur des choix politiques - comportant inévitablement une part d'arbitraire ${ }^{61}$ - mais, connue et reconnue de tous, elle peut impulser des actions tangibles ${ }^{62}$. Ainsi, la comptabilité nationale est l'un des outils servant de fondement à la prise de décisions concrètes par les personnes publiques ${ }^{63}$ parce qu'elle permet d'évaluer la situation patrimoniale et financière des personnes publiques mais aussi de calculer le PIB national, c'est-àdire la création de richesse annuelle sur le territoire. Pour l'heure, ce référentiel ne tient aucun compte des données naturelles. Pourtant, les intégrer aux comptes nationaux permettrait de mesurer le capital naturel d'un Etat mais aussi ses fluctuations. Ceci accroitrait la légitimité des politiques publiques favorables à l'environnement et,

59. http://www.cnrtl.fr/definition/convention

60. Attribuer une valeur ne modifie pas la nature du sous-jacent; la comptabilisation n'est pas synonyme de marchandisation, Martin G.-J., "Les "biens-environnements" », op. cit., p. 149 et Centre d'analyse stratégique, Chevassus-Au-Louis et al., op. cit., p. 7.

61. "Que valent la Tour Eiffel, le Château de Versailles et le pont du Gard ? Que valent les Alpes ou la Côte d'Azur ? Et notre système éducatif ? (...) La richesse d'un pays est une opinion », Henochsberg M., " Oui, nous sommes solvables!», Revue Banque, juin 2012, supplément, pp. $21 \mathrm{~s}$.

62. Centre d'analyse stratégique, Chevassus-Au-Louis et al., op. cit., p. 7.

63. Greffe X., Gestion publique, Dalloz, 1999, p. 376. 
a minima, des décisions tendant à lutter contre sa dégradation. Ainsi, quoi qu'il en soit des critiques (1), des propositions de comptabilité verte ont été élaborées (2).

\section{1) Les oppositions à la comptabilité verte}

Cette méthode de protection de la nature suscite de vives oppositions qui proviennent aussi bien des militants écologistes que des défenseurs du libéralisme économique. Les réserves soulevées par les uns et par les autres nous semblent peu fondées. Toutes les résistances, quelles que soient leurs inspirations philosophiques, mobilisent d'abord l'argument de la complexité de l'élaboration d'un référentiel comptable environnemental pour s'y opposer ${ }^{64}$. Trois sous-arguments ont été principalement soulevés : tous les éléments naturels n'ont pas été répertoriés ; leur estimation ne saurait être exacte ; il paraît difficile d'anticiper les dynamiques de ces éléments alors que celles-ci pourraient justement aider à donner des estimations ${ }^{65}$. Cependant, ces difficultés ne devraient pas être paralysantes : malgré le risque d'une estimation imparfaite, l'intégration des données naturelles élargirait l'image que l'on peut avoir de la richesse « véritable » par habitant. A l'argument de la complexité technique, s'ajoutent les réserves propres à chaque courant qui s'oppose à la comptabilité environnementale.

De manière schématique, les écologistes considèrent que l'estimation constitue la première étape vers une marchandisation de la nature qui, in fine, aboutirait au résultat contraire à celui espéré, à savoir une exploitation des ressources. Or, la comptabilisation n'induit pas nécessairement la possibilité de faire commerce de l'élément évalué. De plus, l'environnement fait déjà l'objet d'estimations ; elles ne sont simplement pas systématiques. En effet, en France, le droit admet la valorisation compensatoire dans le cadre de la séquence « éviter, réduire, compenser » et la valorisation indemnitaire lors d'un contentieux ${ }^{66}$; ne manque plus que la valorisation l'on pourrait

\footnotetext{
64. « L'agrégation d'éléments hétérogènes semble possible jusqu'à un certain point en ce qui concerne le capital physique et humain ou certaines des ressources naturelles ", Commission sur la mesure des performances économiques et du progrès social, Stiglitz J., Sen A., Fitoussi J.-P. et al., 2009, p. 68.

65. Hein L. et al., "An Introduction to Ecosystem Accounting", in Routledge Handbook of Ecosystem Services, Potschin M. et al. (dir.), Routledge, 2016, p. 218.

66. Cf. infra Alidor B. « Compensation et services écosystémiques », Jean S. «L'incidence des services écosystémiques en droit de la responsabilité civile ».
} 
qualifier de « tutélaire » qui tendrait à aider les décideurs politiques dans le choix de leurs actions ${ }^{67}$. Pour ce dernier type de valorisation, l'instauration d'une comptabilité verte semble inévitable.

De manière toute aussi succincte, les tenants du libéralisme économique jugent l'intervention des personnes publiques néfaste pour l'équilibre du marché, il convient donc de la limiter. Ils s'opposent ainsi aux arguments renforçant la légitimité d'action des administrations. Il peut leur être répondu que l'élaboration d'un référentiel environnemental serait bénéfique aussi au secteur privé. En effet, les ménages, mais surtout les entreprises ${ }^{68}$, sont de plus en plus sollicités pour concourir à la protection de l'environnement ${ }^{69}$. Ils auraient également besoin de points de repère conventionnels pour s'approprier des possibilités existantes telles que la création d'Obligations Réelles Environnementales (ORE) ${ }^{70}$ ou la conclusion de PSE. De ce point de vue, il aurait même été souhaitable que l'élaboration d'un système comptable vert soit mis en place avant que les autres mesures n'aient été prises. La faiblesse des argumentaires des opposants à la comptabilité verte explique probablement pour partie que des référentiels environnementaux aient tout de même été élaborés.

67. Martin G.-J., « Les “biens-environnements” », op. cit., p. 140.

68. Dès le sommet de la Terre de Rio, l'application de la comptabilité verte aux sociétés était préconisée, Trébulle F.-G., op. cit., §40. L'intégration de la comptabilité verte aux comptes des personnes privées serait d'autant plus facile que cette dynamique a déjà débuté. D'une part, les entreprises cotées sont dans l'obligation de fournir des informations environnementales depuis la loi Grenelle 2 ; donc elles doivent identifier leurs éléments naturels. D'autre part, certaines appliquent volontairement les référentiels verts internationaux, essentiellement le SEEA de l'ONU, Teller M., « Développement durable et comptabilité », Cahiers de droit de l'entreprise, n 3, Mai 2010, dossier 15.

69. Commission des comptes et de l'économie de l'environnement, Les comptes de l'environnement en 2013, déc. 2015, p. 15, p. 16 et p. 18.

70. Par exemple, la CDC biodiversité a investi pour obtenir des unités de compensation mais pour l'heure, elle ne trouve pas suffisamment d'acteurs économiques demandeurs pour les acquérir. Dans le fameux exemple de la restauration de la biodiversité à Cossure, la Caisse a choisi la stratégie de la compensation par l'offre mais elle n'a réussi à vendre que la moitié des unités de compensation produites sur le site, Calvet C., Levrel H., Napoleone C. et Dutoit T., «La réserve d'actifs naturels : une nouvelle forme d'organisation pour la préservation de la biodiversité en France? », in Restaurer la nature pour atténuer les impacts du développement, Analyse des mesures compensatoires pour la biodiversité, Levrel H et al. (dir.), Quae, 2015, p. 151. Si les causes de ce résultat sont sans doute multiples, l'une d'entre-elles réside dans le fait que le marché est actuellement illisible. 


\section{2) Les propositions de référentiels verts}

Les modalités diffèrent d'une initiative à l'autre mais globalement, la démarche est toujours la même : il s'agit de faire un inventaire des données physiques puis de les transformer en données monétaires. « Le capital naturel peut être considéré comme une partie du stock du capital utilisé pour la production, au même titre que les biens d'équipement, les technologies disponibles et les connaissances humaines. La variation de ce stock représente les changements intervenants entre deux "états de la nature". Elle est obtenue en agrégeant les flux entrants (amélioration des capacités de régénération des systèmes naturels, investissement dans la protection de l'environnement) et les flux sortants (surexploitation des ressources, altération des capacités de régulation des systèmes naturels). Si on est économiste, on appelle les flux entrants "investissements" et les flux sortants "amortissements" ! $"{ }^{71}$. Concrètement, les projets les plus aboutis ont été menés d'abord au niveau international, puis européen.

Dès 1987, 1'ONU a commandé le rapport Brundtland, inspiré par l'article de Nordhaus et Tobin ${ }^{72}$. Ledit rapport relève que les référentiels de l'époque sont incomplets parce qu'ils n'intègrent pas les ressources de l'eau, de la mer et de la $"$ terre $\rangle^{73}$. Puis, cinq années plus tard, à la conférence internationale de Rio, a été défendue " l'idée selon laquelle il est nécessaire de développer une comptabilité environnementale et repenser les systèmes de comptabilité nationale [pour], à terme, permettre de comptabiliser "toutes les contributions économiques et sociales qui ne sont pas comptabilisées dans les comptes nationaux conventionnels" $\gg\rangle^{74}$. En plus d'inciter les Etats à aller dans cette direction ${ }^{75}$, l'ONU a

71. De Perthuis C. et Jouvet P.-A., op. cit., p. 68.

72. Dès les années 1970, ces auteurs proposaient de calculer le Measure of Economic Welfare (MEW) pour ajuster le PIB en y ajoutant les loisirs, les tâches ménagères non rémunérées, mais aussi les externalités négatives telles que la pollution, Nordhaus W. D. and Tobin J., "The Measurement of Economic and Social Performance », in Is Growth Obsolete?, NBER, 1973, pp. 509-564.

73. UN report, Brundtland et al., Our Common Future, 1987, p. 47. Ce travail précise que, pour chacun de ces milieux, les comptabilités nationales devraient inclure - entre autres les actifs que représentent la variété des espèces et des gènes, c'est-à-dire, la biodiversité, ibid., pp. 125- 135.

74. Trébulle F.-G., « Droit du développement durable », J.-Cl. Env. et DD, fasc. 2400, §40. 75. Voir la sous-section d) du chapitre 8 de l'agenda 21 ; https://sustainabledevelopment. un.org/content/documents/Agenda21.pdf 
commencé à élaborer son propre référentiel comptable vert, le System of Envionmental-Economic Accounts (SEEA), pour leur servir de base de travail. Entre sa première version - en 1993 - et la dernière parue une décennie après, le SEEA a progressivement été affiné. Sa dernière mouture se concentre justement sur l'estimation des écosystèmes ${ }^{76}$, ceux-ci comprenant les sols ${ }^{77}$ et leurs services. Ce travail a servi de base de réflexion au niveau européen.

En 2011, l'Union a lancé la « Stratégie Europe 2020 » qui comprend une liste d'actions à mener dont l'instauration d'un référentiel vert ${ }^{78}$, intégrant là encore la valeur des sols et des services qu'ils rendent ${ }^{79}$. Eurostat est donc actuellement en voie de consolidation d'un système de comptabilité environnementale appelé Knowledge Innovation Project - Integrated system for Natural Capital and ecosystem services Accounting (KIP-INCA). Il est encore aujourd'hui à l'état de projet et ne devrait aboutir qu'à l'issue de la Stratégie, soit en 2020. Pour accélérer le processus, Eurostat s'est basé sur des travaux déjà réalisés par d'autres entités et a ainsi pu bénéficier de synergies. Pour la première phase, celle de l'inventaire physique, l'institut statistique a collaboré avec des agences européennes qui avaient effectué le travail de cartographie des éléments naturels sur le territoire continental ${ }^{80}$. Pour la phase d'estimation des éléments recensés, Eurostat s'est inspiré du SEEA de l'ONU ${ }^{81}$. Comme le

76. UN, System of Environmental-Economic Accounting 2012, Experimental Ecosystem Accounting, CO-edited with the European Commission, the Organisation for Economic Co-operation and Development and the World Bank, 2013.

77. Pour chaque unité écosystémique sont valorisés la faune, la flore, le sol, l'eau et le carbone en fonction de leurs caractéristiques (variété, quantité, etc), UN, System of Environmental-Economic Accounting 2012, op. cit, p. 50.

78. EU reference document on Natural Capital Accounting, op. cit., p. 7.

79. European commission, Knowledge innovation project (KIP) on Accounting for natural capital and ecosystem services, Scoping paper, June 2015, p. 3.

80. Il a notamment - mais pas seulement - collaboré avec l'Agence Européenne de l'Environnement (AEE), le Enhancing ecoSysteM sERvices mApping for poLicy and Decision mAking (ESMERALDA) et le Mapping and Assessment of Ecosystems and their Services (MAES) chargés de répertorier, de géolocaliser et d'évaluer les écosystèmes sur le territoire de l'UE, l'Operational Potential of Ecosystem Research Applications (OPERAs) qui a pour mission de proposer des stratégies concrètes d'application des connaissances universitaires sur la biodiversité et les écosystèmes, ainsi que le Land Use and land Cover Survey (LUCAS), un autre programme d'Eurostat spécialisé dans l'étude des sols, European commission, Knowledge innovation project (KIP) on Accounting for natural capital and ecosystem services, Scoping paper, op.cit., p. 1.

81. Ibid., p. 2. D'ailleurs, KIP-INCA est aussi censée inspirer des initiatives nationales. Ainsi, la France a préparé une « Stratégie Nationale pour la Biodiversité » (SNB) courant sur la même période que la stratégie européenne. Elle reprend les objectifs et actions 
référentiel international, KIP-INCA a une conception large du capital naturel qui devrait inclure : les écosystèmes comme les forêts, les rivières ou encore les parcs urbains, les services écologiques qu'ils fournissent, qu'il s'agisse des services d'approvisionnement, de régulation et de maintenance ou les services culturels, les actifs abiotiques épuisables et non épuisables ${ }^{82}$. Soulignons cependant que la proposition qui a été synthétisée dans le tableau ci-dessous, ne constitue qu'un document de travail très sommaire d'Eurostat. Elle reste perfectible, et gagnerait par exemple à inclure davantage les scientifiques spécialisés dans le champ de la biologie pour affiner les catégories d'écosystèmes, de services, d'actifs et de flux et ainsi se rapprocher de la réalité observable ${ }^{83}$.

européens pour les mettre en œuvre au niveau national et local. Le document ministériel qui en dessine les grands axes, prévoit ainsi que « les valeurs de la diversité biologique » soient incorporées aux comptes nationaux au terme de cette période afin que la préservation de la biodiversité devienne « un enjeu économique positif pour les décideurs ». Or, la Stratégie inclut les sols, Premier ministre, Stratégie nationale pour la biodiversité 2011-2020, août 2012, spéc. pp. 7, 14 et 51. Cette intégration paraît d'autant plus pertinente que depuis la loi RBNP, l'article L. 110-I du Code de l'environnement reconnaît que « les espaces, ressources et milieux naturels terrestres et marins, les sites, les paysages diurnes et nocturnes, la qualité de l'air, les êtres vivants et la biodiversité font partie du patrimoine commun de la nation [et que] ce patrimoine génère des services écosystémiques et des valeurs d'usage ». L'article L. 110-I al. 2 ajoute que « les processus biologiques, les sols et la géodiversité concourent à la constitution de ce patrimoine ». Malgré ces avancées, le travail au niveau national reste encore peu développé. Dans leur dernier rapport sur l'application du droit environnemental européen, les sénateurs ne font que retranscrire l'annexe d'une Communication de la Commission enjoignant la France à « poursuivre l'action en faveur de la cartographie et de l'évaluation des écosystèmes et des services écosystémiques ainsi que l'évaluation et le développement des systèmes de comptabilité du capital naturel », (Sénat, Commission des finances, rapport n ${ }^{\circ} 605$, Husson J.-F., L'application du droit européen de l'environnement, juil. 2017, p. 79) sans apporter d'éléments nouveaux quant à réalisation d'un référentiel français.

82. Eurostat, Compilation of comments on draft Natural Capital reference document, Feb. 2015, p. 11.

83. A titre d'illustrations voici quelques remarques qui ont été formulées par les agronomes participant au présent projet et montrant les limites du référentiel en l'état actuel : évidemment, les ressources marines sont surexploitées mais les océans sont compris comme des écosystèmes épuisables alors que l'eau, sa principale composante, devrait voir sa masse augmenter ; pareillement, l'ozone est considéré comme épuisable alors qu'il se régénère à haute altitude et le « trou » observé dans les années 1980 se résorbe ; au contraire, l'atmosphère devrait apparaître de le tableau comptable. 


\section{CAPITAL NATUREL}

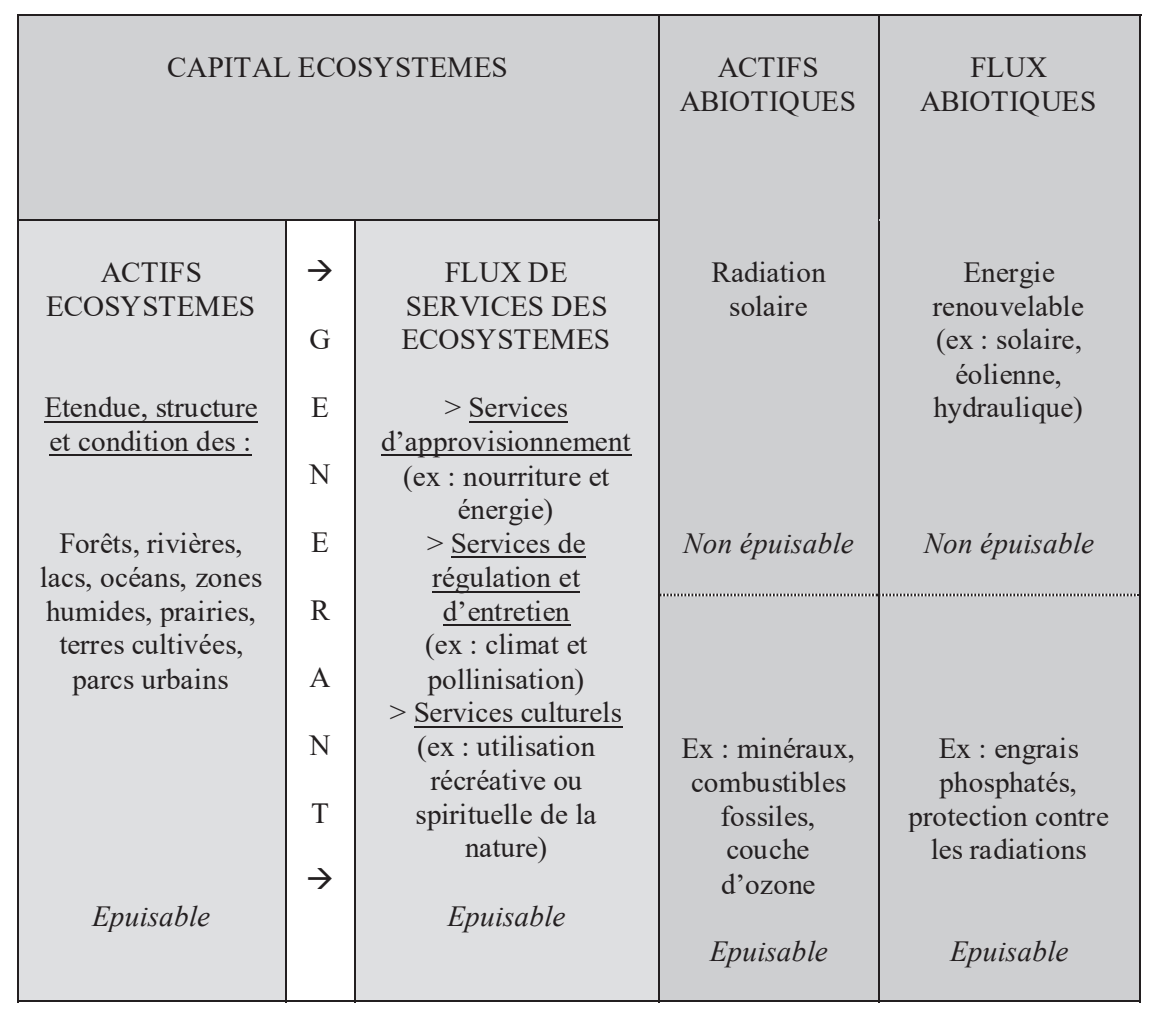

Source : EU reference document on Natural Capital Accounting, Prepared as part of the EU MAES process, January 2015, figure 2.1, p. 11. - traduction de l'auteure

En dépit de ces défauts de conception à son actuel stade de développement, la construction d'une telle convention comptable semble tout à fait indiquée pour sauvegarder les sols parce qu'elle a une vision large de la « nature » incluant entre autres leur valeur, dans tous les types d'écosystèmes, leur biodiversité (sans distinction entre celle qui dite " ordinaire » et celle dite "remarquable ${ }^{84}$ ), et l'ensemble de leurs productions et fonctions, qu'elles nécessitent une action humaine ou non. De plus, puisqu'il ne s'agit finalement que d'une réflexion, l'élaboration d'un référentiel ne requiert pas

84. La différence peut être faite pour faciliter le travail de comptabilisation en procédant par sous-ensemble (Centre d'analyse stratégique, Chevassus-Au-Louis B. et al., op. cit., p. 7) mais le référentiel prendrait les deux en compte. 
au préalable l'adoption de norme juridique : les échecs successifs de création de règles contraignantes pour protéger ce milieu ${ }^{85}$ ne constitue pas un frein à la comptabilisation ; mieux encore, cette démarche pourrait conduire le législateur - européen et français - à mesurer les enjeux de la protection des sols, le colt de leur « non protection » et à voter, à l'avenir, des dispositions visant à les préserver.

Le travail nécessaire à l'élaboration d'un nouveau référentiel est colossal en raison, à la fois de la complexité du fonctionnement de l'environnement, et de la quasi-absence de marché permettant d'indiquer la valeur de la nature. Cependant, face à ces difficultés, le potentiel qu'offrirait un tel système comptable pour protéger l'environnement et particulièrement les sols et leurs services, mérite de fournir cet effort intellectuel.

B. LES INTÉRÊTS BUDGÉTAIRES DE LA COMPTABILITÉ ENVIRONNEMENTALE ET LE VERDISSEMENT PROGRESSIF DE LA DÉPENSE PUBLIQUE

L'adoption d'un référentiel comptable vert paraît particulièrement adaptée pour encourager la protection de l'environnement en général, des sols en particulier. Elle serait utile aux personnes publiques qui pourraient mieux évaluer leurs richesses naturelles et ainsi engager des fonds publics pour les préserver. Pour instaurer une comptabilité environnementale qui influence concrètement la décision publique, il faudrait suivre ces étapes : commencer par réfléchir à un référentiel en dehors d'une obligation normative - tel que l'Union le fait actuellement avec le projet KIP-INCA puis, l'intégrer à la comptabilité nationale. En effet, cette dernière a acquis une valeur obligatoire dans la mesure où les Etats se sont engagés à tenir une telle comptabilité puis à transmettre ces données aux institutions européennes dans le cadre de la discipline budgétaire. Ainsi, chaque année, la France doit calculer son PIB, la dette et le déficit publics ${ }^{86}$ puis la Commission contrôle le respect des règles encadrant les finances publiques nationales. De nombreuses contraintes ont été définies mais les deux plus connues sont les limites posées à l'endettement qui ne devrait pas

85. Cf. supra Hermon C. « La protection du sol en droit».

86. Annexe B du règlement (UE) $n^{\circ}$ 549/2013 du Parlement européen et du Conseil du 21 mai 2013 relatif au système européen des comptes nationaux et régionaux dans l'Union européenne. 
dépasser $60 \%$ du PIB, et au déficit qui ne devrait pas excéder $3 \%$ du même montant ${ }^{87}$. Ainsi, mécaniquement, si les richesses naturelles étaient incluses au PIB, celui-ci augmenterait et les ratios de discipline budgétaire s'en trouveraient réduits. Pour l'exercice 2016, la France avait un taux d'endettement de $96 \%$ et un déficit de $3,4 \%{ }^{88}$. Intégrer les éléments naturels - dont les sols et leurs services - sachant que la France a un patrimoine naturel très riche en raison de son positionnement continental ${ }^{89}$ et de ses terres outre-mer ${ }^{90}$, permettrait très certainement à $1^{\prime} E$ tat de respecter ses obligations européennes et lui laisserait même une marge de manœuvre financière plus importante ${ }^{91}$. Là encore, le caractère conventionnel, et par là même quelque peu artificiel, inhérent à tout système comptable apparaît clairement. Adopter un référentiel constitue indéniablement un choix politique. Mais cette remarque vaut tout autant pour la comptabilité actuelle que pour la comptabilité verte.

En plus de soulager les personnes publiques des contraintes relatives à leurs dépenses, leurs dettes et leurs déficits, la comptabilité environnementale pourrait permettrait un verdissement du volet fiscal de leurs finances publiques. D'une part, en identifiant les variations affectant les éléments naturels, une comptabilité verte faciliterait aussi le travail de suppression des subventions - autorisées mais néfastes pour l'environnement qui est présentement mené. En effet, l'UE encourage ses membres à éradiquer les aides publiques nuisibles ; à ce titre, la France a commandé un rapport sur la question paru en 2010. Ses recommandations principales visent à mieux répertorier les aides dommageables, ce qui nécessite de «progresser dans la méthode de comptabilisation ${ }^{92}$. D'autre part, toujours grâce aux constats dressés par une comptabilité environnementale, les personnes publiques pourraient réorienter leurs prélèvements

87. Traité de Maastricht, article $1^{\text {er }}$ du protocole $n^{\circ} 12$ sur la Procédure de Déficit Excessif (PDE).

88. https://www.insee.fr/fr/statistiques/2669747

89. Centre d'analyse stratégique, Chevassus-Au-Louis B. et al., et al., op. cit., p. 44.

90. Office National de la Biodiversité (ONB), Bilan 2016 de l'état de la biodiversité en France : une nature française sous tension, mai 2016, p. 1.

91. Ajoutons que l'élaboration d'un référentiel est relativement peu coûteuse dans la mesure où elle induit essentiellement des frais de personnel pour effectuer les recherches théoriques puis la mise en œuvre, Chevassus-Au-Louis B. et al.,., op. cit., p. 265.

92. Centre d'analyse stratégique, Les aides publiques dommageables à la biodiversité, Sainteny G. (dir.), La Documentation française, 2010, pp. 35 s. 
obligatoires dans un sens plus écologique. Cette redirection ne semble pas superflue au regard du double effet pervers de la fiscalité actuelle sur la préservation des sols. D'une part, « la fiscalité locale incite à l'artificialisation du territoire. En effet, la quasi-totalité des recettes fiscales des collectivités territoriales sont assises sur la construction, l'existence ou la transaction d'éléments bâtis ou d'infrastructures $\|{ }^{93}$. D'autre part, l'imposition du patrimoine incite à la mobilité en ce qu'elle pénalise les valeurs refuges - c'est-à-dire les investissements longs et peu risqués - et les biens improductifs (ceux qui ne sont pas utilisés dans le cadre d'une activité économique). Au contraire, les acteurs sont encouragés à choisir une plus forte rentabilité à court terme. Or, les espaces naturels - sols compris sont d'autant plus protégés qu'ils sont exploités moins intensément et qu'ils restent immobiles.

Ainsi, malgré les oppositions politiques qui continuent de freiner le développement et l'utilisation d'un référentiel comptable environnemental, la France s'achemine vers l'intégration des éléments naturels dans la prise de décision en matière de finances publiques. Plusieurs illustrations pourraient être présentées mais deux d'entreelles apparues très récemment - l'une concernant l'Etat et l'autre concernant les collectivités territoriales - sont particulièrement éclairantes en termes d'encouragement de la dépense publique à des fins de protection de l'environnement. Elles s'appliquent entre autres à la préservation des sols et de leurs services.

Alors même que le PIB ne comprend pas encore les richesses naturelles, l'Etat emprunte - augmente sa dette - spécifiquement pour engager des dépenses vertes. En effet, il vient de créer un titre obligataire, l'Obligation Assimilable du Trésor (OAT) verte ${ }^{94}$, qui indique à ses acquéreurs sur les marchés de capitaux, que les fonds mis à sa disposition couvriront obligatoirement des frais pour protéger l'environnement. Ces capitaux seront utilisés pour lutte contre le changement climatique et contre la pollution mais aussi pour la protection de la biodiversité ${ }^{95}$. Dans le communiqué de presse de l'Agence France Trésor (AFT) annonçant cette nouveauté, l'OAT verte a été présentée comment un produit financier rendant

93. Sainteny G., Plaidoyer pour l'écofiscalité, Buchet Chastel, 2012, p. 100.

94. Suite à la COP 21, la ministre de l'Environnement et le ministre de l'Économie et des Finances s'étaient engagés à créer des « Green Bonds ».

95. République française, Document-cadre de l'OAT verte, 10 janvier 2017. 
la place boursière de Paris plus attractive pour les investisseurs. D'ailleurs, l'émission inaugurale du 23 janvier 2017 a rencontré un grand succès auprès des investisseurs puisqu'ils ont consenti à prêter à la France 7 milliards d'euros ${ }^{96}$ à $1,75 \%$ pour une durée de vingtdeux ans ${ }^{97}$.

Concernant l'échelon local, l'article 4 de la loi « montagne » de décembre $2016^{98}$, ajouté sur amendement sénatorial, énonce que la péréquation tiendra désormais compte à la fois des ressources naturelles, dont les « services, notamment écologiques et environnementaux ", et des frais financiers qu'engendrent ces espaces pour les collectivités territoriales ${ }^{99}$. Il organise à la fois une péréquation verticale et horizontale en faveur des personnes publiques dont les charges liées à la préservation des territoires de montagne grèvent le plus lourdement le budget ${ }^{100}$ et dont les territoires procurent des services à tous. Bien que le mécanisme prenne aussi en compte la valeur des services rendus par la nature, sa présentation dans les rapports parlementaires insiste davantage sur l'aspect charge financière parce que les territoires montagneux sont en moyenne des espaces à plus faible densité démographique qui, par là même, disposent de ressources fiscales réduites ${ }^{101}$. Dans le même temps,

96. Le montant total des dépenses vertes éligibles identifiées pour les émissions de l'AFT au cours de l'année 2017 est supérieur à 10 milliards d'euros ; chaque euro levé trouvera donc un emploi.

97. http://www.aft.gouv.fr/articles/lancement-de-1-oat-verte-1-75-25-juin-2039 12865. html

98. Loi n 2016-1888 du 28 décembre 2016 de modernisation, de développement et de protection des territoires de montagne.

99. Précisément, « la dotation globale de fonctionnement et le fonds national de péréquation des ressources intercommunales et communales intègrent les surcoûts spécifiques induits par des conditions climatiques et géophysiques particulières en montagne et les services, notamment écologiques et environnementaux, que la montagne produit au profit de la collectivité nationale ».

100. En vertu de 1'article 72-2 al 4 de la Constitution, « la loi prévoit des dispositifs de péréquation destinés à favoriser l'égalité entre les collectivités territoriales ». La péréquation verticale correspond à une dotation globale de fonctionnement de l'Etat supplémentaire attribuée aux collectivités locales les plus faibles et la péréquation horizontale au transfert d'une fraction des ressources fiscales des collectivités les plus favorisées vers ces collectivités.

101. L'Association Nationale des Elus de la Montagne (ANEM) et la Banque postale ont réalisé en 2016, pour la première fois, une étude spécifique sur cette question. Il en ressort que « les charges financières par habitant sont supérieures de $29 \%$ par rapport à la moyenne nationale pour les communes de moins de 1000 habitants. Ces dernières représentent $79 \%$ des communes de montagne. Ce ratio confirme l'existence de surcoûts spécifiques à la montagne - liés à l'altitude, au climat et à la pente », ANEM, "Les finances des communes de montagne à la loupe », Pour la montagne, n² 277, Décembre 2016, p. 2. 
la préservation de la nature - notamment le bon fonctionnement des écosystèmes et la sauvegarde des sols - profitent à l'ensemble de la communauté nationale ${ }^{102}$. Le principe est adopté mais les modalités pratiques de mise en œuvre n'ont pas encore été rendues publiques ${ }^{103}$. Il faudra donc attendre l'avenir pour savoir concrètement comment la péréquation fonctionnera.

Ces deux exemples d'intégration lente mais croissante de la question environnementale dans les finances publiques françaises nous semblent résulter de ce que les oppositions à la comptabilité verte sont fragiles mais surtout des atouts que présentent cette méthode de protection de la nature. L'édiction d'un référentiel vert est particulièrement séduisante dans le cadre de la sauvegarde des sols. Contrairement à la protection immédiate des services des sols reposant sur les PSE, cette solution n'est pas limitée par le consentement des acteurs privés, permet une valorisation plus large que celle des seuls services écologiques et étend le champ de la préservation des sols au-delà de l'activité agricole. La voie paraît donc pertinente.

Ce problème avait déjà été relevé par la Cour des comptes qui, avant l'adoption de ce texte, intégrait le critère « zone de montagne » à ses analyses en matière de finances locales, Cour des comptes, Concours financiers de l'Etat et disparités de dépenses des communes et de leurs regroupements, octobre 2016, par exemple p. 62. La Cour n'a pas rédigé de rapport depuis le vote de la loi.

102. Actu environnement, rapport rédigé par Mmes Genevard A. et Laclais B., Un acte II de la loi montagne pour un pacte renouvelé de la Nation avec les territoires de montagne, juillet 2015, p. 73. Ces deux députées ont également été rapportrices sur le projet de la loi. 103. L'échéancier des décrets d'application ne prévoit pas de texte spécifique pour la mise en œuvre de l'article 4. 
Service écosystémique et droit des contrats 



\title{
Services écosystémiques et contrat Quelle obligation contractuelle environnementale ?
}

\author{
Matthieu POUMARÈDE \\ Professeur, Université Toulouse Capitole \\ IEJUC, F-31000 Toulouse
}

\begin{abstract}
Appréhender la manière dont le contrat, de droit privé ${ }^{1}$, peut saisir les services écosystémiques suppose, au premier chef, de déterminer s'ils peuvent être l'objet d'une obligation, c'est-à-dire s'ils peuvent être qualifiés de " prestation » au sens de l'article 1163 du Code civil. Autrement dit, existe-t-il un contrat de prestation de service écosystémique, contrat par lequel un débiteur (le propriétaire d'un fonds, son exploitant) s'engagerait à exécuter un service écosystémique au profit d'un créancier? Répondre à cette interrogation, suppose que le service écosystémique soit préalablement qualifié.
\end{abstract}

Essayant nombre de qualifications juridiques, Guillaume Beaussonie $^{2}$ a posé l'hypothèse suivante : un service écosystémique serait une utilité d'un bien propre. Une telle qualification permettrait de concilier protection de l'environnement en tant que tel et pour l'intérêt qu'il présente pour l'homme. Elle mériterait donc d'être confrontée à l'ensemble des services écosystémiques dont on sait l'immense diversité, même classifiée. Particulièrement, si elle peut seoir aux services de régulation et de maintenance, son adéquation aux services d'approvisionnement devrait être éprouvée ${ }^{3}$, alors que

1. Sur la contractualisation avec une personne publique, v. Labous K. et Gruger H., «Produire de la biodiversité : un avenir pour les agriculteurs », Dr. Env., 2017, 291.

2. Beaussonie G., «La qualification juridique des services écosystémiques », supra.

3. Sur cette question, v. notamment Langlais A., «L'appréhension juridique de la qualité des sols agricoles par le prisme des services écosystémiques », Dr. rural 2015, Etude 20. 
la qualification de fruits (C. civ., art. 583 et s. ${ }^{4}$ ), voire de produits, paraît mieux leur convenir ${ }^{5}$.

Quoi qu'il en soit, ainsi compris, telles des utilités, les services écosystémiques pourraient-ils être l'objet d'une obligation contractuelle? L'article 1163 du Code civil disposant que « l'obligation a pour objet une prestation présente ou future $»$, ne semble pas, au premier abord s'y opposer, la prestation pouvant s'entendre, notamment, d'un service ${ }^{6}$ écosystémique. Néanmoins, en quête d'une qualification, Guillaume Beaussonie après avoir montré que le service est une utilité d'un bien propre précise, aussitôt, qu'il s'agirait d'une utilité commune ${ }^{7}$. Aussi, comme telle, elle bénéficierait à tous et non au seul propriétaire du bien, pas davantage, sans doute qu'à un seul et unique créancier ou à un groupe de créanciers identifiés ${ }^{8}$. En conséquence, le service écosystémique paraît rétif à être l'objet d'une obligation contractuelle unissant le seul propriétaire du bien à un tiers, qu'il soit personne privée ou publique9.

Plus avant, la définition du service écosystémique rejoint (naturellement) cette qualification juridique mise en avant par Guillaume Beaussonie, notamment, par opposition à la notion de service environnemental. Le premier, le service écosystémique, est un « avantage socioéconomique retiré par l'homme de son utilisation durable des fonctions écologiques des écosystèmes $»^{10}$ ou si l'on préfère, un « service rendu par l'ecosystème $»^{11}$.

\footnotetext{
4. V. intervention de Vanuxem S., «La nature des services écosystémiques en droit privé », IDEX T2SEC, IEJUC, Toulouse, 26 mai 2016.

5. Et, partant, la contractualisation des services d'approvisionnement ne semble ainsi guère poser des difficultés particulières, du moins mal connues.

6. Chantepie G. et Latina M., La réforme du droit des obligations, Dalloz, 2016, art. 1163.

7. Beaussonie G., préc.

8. Comp. sur la question de la propriété des services et pour une position assez proche (sous un autre angle), Maris V., Nature à vendre, Les limites des services écosystémiques, Quae éd., 2014, p. 60 ; de manière plus générale, voir Deffairi M., La patrimonialisation en droit de l'environnement, IRJS éd., 2015, p. 639 et ss. et les réf. citées.

9. Comp. Langlais A., «Les paiements pour services environnementaux comme nouveau contrat environnemental », in Boutonnet M. (dir.), Le contrat et l'environnement, Etude de droit interne, international et européen, PUAM, 2014, p. 185, sp. p. 191

10. V. Glossaire, infra.

11. Langlais, A., sp. p. 186
} 
Autrement dit, aucune personne juridique ne semble pouvoir être débitrice d'un service écosystémique ${ }^{12}$; du moins si l'on admet que les personnes juridiques ne produisent ni même ne coproduisent des services écosystémiques. Après tout, la culture favorisant par exemple la pollinisation, l'on pourrait s'accorder à considérer que la personne juridique en est le coproducteur. Mieux, cela parait encore plus évident s'agissant des services d'approvisionnement : un fruit n'est-il un « coproduit » de l'écosystème et de la personne juridique? Toutefois, y compris dans cette dernière hypothèse, les services d'approvisionnement, nous retiendrons que le service demeure écosystémique, en ce sens que si les personnes juridiques participent manifestement au fonctionnement de l'écosystème, si elles peuvent agir en sa faveur, elles ne le fournissent pas et ne peuvent donc en être, directement, débitrices ni codébitrices. Néanmoins, avant de poursuivre, il peut être souligné que s'il devait être retenu que les personnes juridiques coproduisent des services, certaines solutions ci-après développées devraient être en partie reconsidérées.

De même, et corrélativement, une personne juridique (identifiée) ne semble pas pouvoir être créancière, au sens juridique, d'un service écosystémique. Même si, à nouveau, les hésitations sont permises, le bénéfice des services écosystémiques aux personnes juridiques semble devoir être factuel. Que les personnes en retirent un " avantage » est certain, quand bien même cela met en relief une approche anthropocentrée de l'environnement. Mais cet avantage ne peut constituer une créance au profit d'une personne juridique identifiée. Par exemple, si tel service écosystémique (pollinisation des cultures, contrôle des ravageurs) bénéficiera potentiellement davantage au " voisin », de part cette qualité, il n'en est pas créancier, faute de débiteur. Simplement, il en tire avantage, ainsi que l'humanité.

12. V. également Langlais A., «Les paiements pour services environnementaux, une nouvelle forme d'équité environnementale pour les agriculteurs ? Réflexions juridiques », Dr. rural 2013, Etude 7 : « dans de nombreuses hypothèses, la réalité de l'objet du contrat semble aléatoire parce que la complexité de l'écosystème rend difficile l'identité parfois mais surtout l'identification du service rendu. Cette difficulté peut également provenir des caractères du service rendu attendu : ce service peut se traduire en une amélioration environnementale à plus ou moins long terme ou bien encore en termes d'atteinte évitée. Or, cette condition est un préalable indispensable pour s'assurer de la réalité de l'engagement». 
Produit de l'écosystème, le service écosystémique bénéficie à une collectivité, voire à l'humanité, loin d'un rapport contractuel obligeant un débiteur (le propriétaire) et un créancier (un bénéficiaire) auquel il ne peut donc être réduit ; ce faisant le spectre de la prétendue marchandisation de la nature s'éloigne, en même temps, revers de cette médaille, que la possibilité d'obliger une personne à rendre un service écosystémique. Il ne peut en effet être l'objet d'une obligation contractuelle. Peut-être est-ce ce que le législateur suggère même si les conventions de vocabulaire étant encore mal assurées ${ }^{13}$, il convient d'être prudent. En effet, les « services écosystémiques » sont les grands absents tant de la compensation, concernant les « atteintes qui n'ont pu être évitées ni réduites, en tenant compte des espèces, des habitats naturels et des fonctions écologiques affectées » (C. envir., art. L. 110-1), que de l'obligation réelle environnementale qui n'a que pour finalité « le maintien, la conservation, la gestion ou la restauration d'éléments de la biodiversité ou de fonctions écologiques » (C. envir., art. L. 132-3).

Si le service écosystémique ne peut être l'objet d'une obligation contractuelle, c'est-à-dire une prestation, faut-il en conclure que le contrat ne serait pas apte à le saisir autrement ? Une telle conclusion serait surprenante alors que le contrat s'est d'ores et déjà " imposé comme mode de protection de l'environnement aux côtés de moyens originellement plus classiques tels que le droit imposé $\gg{ }^{14}$. Et précisément, il est observable que les personnes juridiques, le propriétaire du bien au premier chef voire le preneur et plus généralement l'exploitant, peuvent être en position d'agir sur la quantité ou la qualité de ces services. En effet, il est certain que l'Homme peut rendre des services à la nature. En préservant ou améliorant l'environnement, il favorise alors l'écosystème ${ }^{15}$ et

13. V. par ex. les observations de Hermon C. au sujet de la référence aux « biens publics environnementaux », aux « services environnementaux » ou « services écosystémiques » fournis par l'agriculture, «Les travaux préalables de la Commission européenne et les textes portant réforme de la PAC », in "Agriculture et environnement. Un nouveau projet pour la PAC? », Revue de l'Union Européenne 2014, p. 52.

14. Langlais A., préc., p. 199. Comp. dans un domaine voisin Giraudel C., « Un phénomène nouveau, le développement des conventions et des partenariats privés ", in la protection conventionnelle des espaces naturels, PULIM, 2000, p. 13. Adde Monteillet V., La contractualisation du droit de l'environnement, Dalloz, coll. Bibliothèque des thèses, 2017. 15. V. Glossaire : « complexe dynamique formé de communautés de plantes, d'animaux et de micro-organismes et de leur environnement non vivant qui, par leur interaction, forment une unité fonctionnelle ». 
sa capacité à fournir des services ${ }^{16}$. Tel est le propos des services environnementaux, " services que des acteurs (...) rendent à la société dans son ensemble (...) et qui visent à réduire la pression exercée sur les écosystèmes ou qui améliorent leur fonctionnement » : autrement dit il s'agit cette fois-ci des « services rendus par l'Homme à la nature $\gg{ }^{17}$. A titre préventif ou réparateur, les personnes juridiques peuvent ainsi rendre un service environnemental améliorant le fonctionnement d'un écosystème en favorisant les services et produits.

Ainsi, mieux que le service écosystémique, c'est le service environnemental qui semble apte à prendre les traits de l'objet d'un devoir, voire d'une obligation si un créancier peut être identifié. En effet, le service environnemental est avant tout un service rendu à la société dans son ensemble ; cela justifie au moins, une approche institutionnelle, vecteur d'un sens commun. Mais, dès lors qu'un débiteur peut être identifié, la contractualisation du service environnemental est possible : il s'agit de contractualiser des pratiques agricoles ou des systèmes de production agricole vertueux en termes d'environnement ${ }^{18}$; le service environnemental est alors du point de vue juridique une " prestation $\|^{19}$, c'est-àdire l'objet d'une obligation contractuelle (art. 1163 C. civil) qui contribue à préserver ou améliorer un écosystème, et donc, les services écosystémiques. Une personne juridique, le plus souvent le propriétaire mais également le preneur (et plus généralement l'exploitant) ainsi que le montre Didier Krajeski ${ }^{20}$, est alors débitrice de ce « service environnemental » (la prestation) au profit d'un

16. Langlais A., « Les paiements pour services environnementaux comme nouveau contrat environnemental », préc..

17. Langlais A., préc..

18. Contra Doussan I. et Martin G. J., «Les PSE à la lumière de la théorie générale des contrats ", in Langlais A. (dir.), L'agriculture et les paiements pour services environnementaux : quels questionnements juridiques?, Presses universitaires de Rennes, 2018, à paraître. Les auteurs distinguent les pratiques agricoles auxquels s'engagent les débiteurs des services environnementaux qui en seraient le produit : " L'agriculteur s'engage à respecter telle pratique culturale, à limiter tel ou tel intrant, à exclure certains modes d'action ou certains produits, comportements dont on attend et on espère qu'ils produiront des services environnementaux. L'observation est capitale : le service environnemental n'est pas, dans ces hypothèses, l'objet de la prestation de l'agriculteur. »

19. Sur la définition du contenu de cette prestation au regard des règles propres à la qualité d'agriculteur, v. Labous K. et Gruger H., préc..

20. Krajeski D., « Travail du sol, services écosystémiques, et bail rural », infra. 
créancier, personne privée ou publique ${ }^{21}$ identifiée par le contrat ; ce service environnemental aura alors pour objectif, notamment, de préserver ou restaurer un écosystème et donc, in fine, un service écosystémique. Ce dernier, s'il ne peut être objet d'une obligation est le but du contrat (art. 1162 C. civil). Par « but» du contrat, il convient de comprendre que le service écosystémique est la cause subjective du contrat (anc. art. 1133 C. civ.), celle en vue de laquelle les parties ont contracté. Par exemple, l'objet de l'obligation principale d'une convention de pollinisation ${ }^{22}$ est la mise à disposition de ruches et des colonies d'abeilles (le service environnemental) et leur entretien : il s'agit alors d'intervenir sur un bien afin d'en permettre son usage agricole et/ou environnemental qui sera maintenu en l'état ou amélioré. En effet, le service de pollinisation, service écosystémique, est la cause subjective de cette prestation ; le «but » doit-on désormais dire (art. 1162 C. civ.). Elle n'en est pas l'objet et ne peut l'être dès lors qu'elle est une utilité commune du bien.

C'est donc en considérant que le service environnemental est la " prestation », c'est-à-dire l'objet de l'obligation, principale ou accessoire, tandis que le service écosystémique peut être le « but» du contrat qu'il est possible d'appréhender l'existence d'un contrat de prestation de service environnemental (I) dont le contenu devra être précisé afin qu'il favorise les services écosystémiques (II).

\section{LA CONTRACTUALISATION DU SERVICE ENVIRONNE- MENTAL}

Pour s'assurer de la réalité du contrat de prestation de service environnemental (A), encore faut-il, préalablement, le distinguer des instruments, nombreux, qui sont mobilisés en faveur des services écosystémiques (B).

21. Hoepffner H., « La commande publique de compensation environnementale », infra. 22. Billet Ph., "La convention de pollinisation », in M. Boutonnet (dir.), préc., p. 269 : « le contrat concerne donc plus la mise à disposition de ruches que la pollinisation qui en résulte ». 


\section{A. Du PSE au CONTRAT DE PRESTATION DE SERVICE ENVIRONNEMENTAL}

Fruit d'une approche économique ${ }^{23}$, la qualification célèbre mais générique de « Paiement pour Services Environnementaux » $(\mathrm{PSE})^{24}$, fait fi de la nature contractuelle (ou non) de l'opération de préservation ou de restauration d'un écosystème ${ }^{25}$. Puisqu'il s'agit avant tout de «Payer les agriculteurs pour les services environnementaux $»^{26}$, sont ainsi indistinctement qualifiés de PSE des contrats dont l'obligation principale, ou accessoire, telle une clause environnementale de bail rural, a pour objet un " service environnemental », mais également diverses techniques paracontractuelles conditionnant un « avantage» (aides, indemnisation) à la mise en œuvre de pratiques favorables à l'environnement ${ }^{27}$. Du point de vue du droit, la catégorie PSE est donc hétérogène : il s'agit alors simplement de qualifier divers processus permettant de préserver l'écosystème et plus avant de protéger l'environnement, quelle que soit la nature des techniques juridiques mobilisées : la loi et le règlement y occupent alors une place naturelle aux côtés du contrat.

Aussi, et quand bien même les frontières sont parfois ténues, le contrat demeure singulier. Membre de la famille PSE, il est particulier en ce que les parties au contrat de prestation de service environnemental n'ont, précisément, pas pour simple objectif de se soumettre à la réglementation en vigueur : il ne s'agit ici pas de se conformer à un texte légal ou réglementaire, quand bien même serait-il prévu une contrepartie. Les parties entendent en effet obliger le débiteur, le plus souvent en contrepartie d'une rémunération convenue entre avec le créancier, à exécuter une " obligation environnementale " qui prend les traits, au cas d'espèce, d'un service environnemental ${ }^{28}$. Or, ce service environnemental, objet de l'obligation principale ou

23. Langlais A., « Les paiements pour services environnementaux, une nouvelle forme d'équité environnementale pour les agriculteurs ? Réflexions juridiques », préc..

24. V. Doussan I. et Martin G.-J., « Les PSE à la lumière de la théorie générale des contrats », préc., qualifiant les PSE de « concept mal maîtrisé ».

25. Comp. Doussan I. et Martin G.-J., préc.

26. Rapport FAO, The state of food and agriculture. Paying farmers for environmental services, 2007.

27. Sur les évolutions et convergences des mécanismes, v. Aznar O. et alii, « Mesures agro-environnementales et paiements pour services environnementaux », in $\mathrm{Ph}$. Méral, D. Pesche (coord.), Les services écosystémiques, Repenser les relations nature et société, Quae éd.2016, p. 201.

28. V. Langlais A., « Les paiements pour services environnementaux comme nouveau contrat environnemental », préc., selon laquelle des PSE « peuvent se caractériser par une 
accessoire ne saurait se confondre avec la seule application des lois et des règlements. Il ne peut s'agir, pour le débiteur de s'engager, visà-vis d'un créancier, à respecter les normes légales et règlementaires. L'on peut en effet raisonnablement penser que si tel était le cas, l'obligation serait dépourvue d'objet. L'article 1107 du Code civil le dit au sujet des contrats à titre onéreux : " le contrat est à titre onéreux lorsque chacune des parties reçoit de l'autre un avantage en contrepartie de celui qu'elle procure $\gg$. Le seul respect de la loi et des règlements ne semble pas pouvoir s'analyser en un avantage : il s'agit d'une « contrepartie (...) illusoire » au sens de l'article 1169 du Code civil. Pour que l'obligation ait un objet, l'engagement pris, nécessairement conforme à la loi et aux règlements, doit en être distinct, soit qu'il soit différent (parce que la loi et les règlements ne le prévoient pas) soit qu'il soit plus exigeant au regard de la protection de l'environnement. Dans tous les cas, l'obligation contractuelle environnementale ne saurait donc être celle prévue par les lois et règlements.

Plus avant, l'on peut s'interroger dans des termes voisins : une pratique conforme aux usages, aux bonnes pratiques agricoles peutelle être contractualisée et, éventuellement, rémunérée ? Répondre à cette interrogation implique, au premier chef, que l'on puisse identifier ces bonnes pratiques agricoles, ailleurs dénommées règles de l'art, auxquelles la Politique Agricole Commune fait notamment référence. Au surplus, il convient de se demander si une telle obligation, de respecter les bonnes pratiques agricoles, a un objet au sens de l'article 1163 du Code civil ; le respect des bonnes pratiques agricoles constitue-t-il un « avantage » au sens de l'article 1107 alors même qu'il devrait s'imposer aux exploitants ? Ne s'agit-il pas simplement, là encore, d'une " contrepartie (...) illusoire » (art. 1169 C. civ.) ? La réponse à cette série d'interrogations est essentielle, en ce qu'elle permettra d'identifier la consistance du service environnemental, et donc de dire quand peut être conclu un contrat de prestation de service environnemental à titre onéreux. En effet, de deux choses l'une. Soit toutes les bonnes pratiques agricoles favorables à l'environnement peuvent être qualifiées d' " avantage », alors même que l'exploitant commettrait une faute à ne pas s'y conformer. Dans ce cas, elles peuvent être l'objet d'une

obligation de faire : celle de réaliser des pratiques ou des services environnementaux »; Comp. Doussan I. et Martin G.-J., préc. 
obligation et, ainsi, leur mise en œuvre, rémunérée : elles constituent une contrepartie réelle. Soit, à l'instar du respect de la loi et des règlements, l'obligation de se conformer aux bonnes pratiques agricoles (si elles peuvent être identifiées) n'a pas d'objet ou, vue du créancier, constitue une « contrepartie (...) illusoire ». Alors, un tel contrat serait nul (art. 1169 C. civ.) ${ }^{29}$.

Le contrat n'a donc pas vocation à embrasser toute sorte de pratiques agricoles favorables à l' environnement. En ce qu'elles ne constituent pas des « avantages » au sens de l'article 1107 du Code civil, sont exclues de son champ les prescriptions légales et réglementaires, mais également, dans la mesure où elles peuvent être identifiées, ne devraient pas faire l'objet d'une obligation environnementale (à titre onéreux) les bonnes pratiques agricoles auxquelles les exploitants doivent se conformer : il s'agirait également là d'une « contrepartie (...) illusoire » susceptible d'entrainer la nullité du contrat de prestation de service environnemental. Autrement dit, ce dernier ne peut valablement contractualiser que des pratiques plus favorables à l'environnement que ne le sont le simple respect des lois et des règlements ou la référence aux bonnes pratiques agricoles.

\section{B. Le SERVICE ENVIRONNEMENTAL CONTRACTUALisÉ : L'OBLigation ENVIRONNEMENTALE}

La contractualisation du service environnemental passe par la création d'une obligation environnementale à la charge d'un débiteur. Le plus souvent, sans doute, cette obligation environnementale prendra la forme d'une obligation accessoire à un contrat, tel un bail rural (1). L'on parlera alors, couramment, de clause environnementale. Mais, et sans que sa nature ne change, l'obligation environnementale peut être principale (2), se nichant ainsi au sein d'un contrat de prestation de service environnemental, stricto sensu.

29. Comp. Labous K. et Gruger H., « Produire de la biodiversité : un avenir pour les agriculteurs », Dr. Env. 2017, 291 qui laissent entendre que le « contrat de prestation de services environnementaux » doit conduire les agriculteurs à fournir des "services environnementaux supplémentaires à ceux qu'ils rendent déjà par le seul fait d'exploiter leurs terres ». 
1) L'Obligation environnementale accessoire : la clause environnementale

Cette obligation environnementale peut être assurément accessoire à un contrat en ce sens qu'elle n'est pas l'obligation principale du débiteur par ailleurs preneur à bail, producteur, etc. Il s'agira par exemple d'une clause environnementale prévoyant, à l'occasion d'un contrat de fourniture de biens agricoles, l'absence d'OGM. De même, en va-t-il du bail rural environnemental, c'est-à-dire contenant « des clauses visant au respect par le preneur de pratiques ayant pour objet la préservation de la ressource en eau, de la biodiversité, des paysages, de la qualité des produits, des sols et de l'air, la prévention des risques naturels et la lutte contre l'érosion, y compris des obligations de maintien d'un taux minimal d'infrastructures écologiques, peuvent être incluses dans les baux » (art. L. 411-27 C. rural). Malgré les difficultés juridiques de mise en œuvre qui en réduisent la portée pratique, ainsi que le montre par ailleurs Didier Krajeski ${ }^{30}$, il s'agit ainsi, par le bail rural, d'obliger le preneur à recourir à des pratiques agricoles favorisant le développement de services écosystémiques sur la base des 15 clauses prévues par l'article R. 411-9-11-1 du Code rural et de la pêche maritime sur lesquelles le bailleur s'appuiera pour rédiger les clauses environnementales du bail ${ }^{31}$.

Dans ces hypothèses et d'autres, telle que la conclusion de convention d'occupation temporaire, l'on retrouvera, accessoirement à une obligation principale, une obligation environnementale ayant pour objet un service environnemental dont l'objet, l'étendue et l'intensité variera : absence d'utilisation d'intrants, préservation d'une zone humide, types de fertilisants à utiliser et/ou limitation de produits phytosanitaires, maintien de surface en herbe, dates et techniques de fauches, techniques de travail du sol, protection de la ressource en eau, diversification des assolements, etc.

Il s'agira parfois d'interdiction, le débiteur devant s'abstenir d'utiliser des produits phytosanitaires sur telle parcelle, de mettre en culture telle autre, d'apporter une fertilisation minérale, de pratiquer

30. Krajeski D., préc.

31. Boutonnet M., «Les obligations environnementales et le contrat de bail ", in Boutonnet M. (dir.), Le contrat et l'environnement, Etude de droit interne, international et européen, PUAM, 2014, p. 57. 
l'écobuage, etc. Mais, il pourra tout autant s'agir de prescriptions, tel le maintien de tous les éléments d'aménagement parcellaire d'origine humaine présents sur la parcelle (murets de pierre sèches, haies...), ou des arbres isolés. Plus avant, il s'agira également de prescrire au débiteur des actions telles que l'engagement de maintenir une couverture permanente du sol, maintenir et entretenir des couverts spécifiques à vocation environnementale (jachères florales, bandes enherbées, ...), etc ${ }^{32}$. Ces interdictions et prescriptions, objets d'une obligation accessoire seront donc adaptées et éventuellement précisées par les parties au contrat en fonction de la configuration des lieux mais également des objectifs visés par les parties, étant rappelé que ni les prescriptions légales et réglementaires ni les bonnes pratiques agricoles ne devraient pouvoir être l'objet d'une obligation environnementale qu'elle soit accessoire ou principale.

\section{2) L'obligation environnementale principale}

Le service environnemental peut être l'objet d'une obligation principale. Ici, l'obligation environnementale est l'obligation contractuelle principale du débiteur, qu'il soit le propriétaire, le locataire ou plus généralement tout exploitant de la parcelle : il s'agit alors d'un contrat de prestation de service environnemental, conclu le plus souvent à titre onéreux ${ }^{33}$. Si ce type de contrat environnemental ${ }^{34}$ visant à la préservation et la restauration de l'environnement est sans doute moins fréquent, on en retrouve néanmoins des manifestations, non sans qu'il existe un risque de requalification en bail rural (C. rural, art. L. 411-1). Certaines sont devenues célèbres, tel le cas Vittel étudié à d'innombrables reprises ${ }^{35}$. La société a notamment conclu des contrats environnementaux avec des fermiers dans l'objectif de continuer à exploiter la source en conformité avec la règlementation. Mais, par-delà ces conventions, l'on peut observer nombre de contrats ayant pour objet de l'obligation principale

32. CEREMA, Direction territoriale Méditerranée, Le bail rural à clause environnementales et le paysage " agro-environnemental », juin 2015, http://www.mediterranee.cerema.fr/ IMG/pdf/BRE_Document_Juin_2015.pdf

33. Sur la fixation du prix dans les contrats de prestation de service, v. l'article $1165 \mathrm{du}$ Code civil.

34. Sur la notion de contrat environnemental, v. Boutonnet M., « Le contrat environnemental», D. 2015, 217.

35. V. par ex. Pomade A., « Les paiements pour services environnementaux contribuent-ils à l'émergence d'un "gradient de juridicité" ? ", VertigO - la revue électronique en sciences de l'environnement, Volume 16 Numéro 1, mai 2016. 
un service environnemental : ils se dénomment « convention de gestion », « bail», etc. Certains sont particuliers, tels les conventions de pollinisation ${ }^{36}$. D'autres font partie d'un complexe de conventions. Ainsi que le décrit Bastien Alidor, l'obligation légale de compensation des atteintes à la biodiversité est génératrice de contrats $^{37}$. Or, rien n'interdit que l'objet de l'obligation principale de ces contrats soit un service environnemental dès lors que cela concorde avec les mesures de compensations prédéfinies par l'arrêté d'autorisation. Si ce n'est lorsque le maître de l'ouvrage prend en charge "directement" (art. L. 163-1 II C. env.) les mesures de compensation, après, par exemple, avoir acquis le foncier nécessaire (encore que, même dans ce cas, il pourrait conclure un bail rural environnemental), dans la plupart des cas le recours au contrat est en effet nécessaire. Tel est le cas lorsque, « en écologue peu averti »" il a recours à un " opérateur de compensation », "personne publique ou privée chargée, par une personne soumise à une obligation de mettre en œuvre des mesures de compensation des atteintes à la biodiversité, de les mettre en œuvre pour le compte de cette personne et de les coordonner à long terme » (art L. 163-1 III C. env.). Mais, que le maître de l'ouvrage agisse directement ou par l'intermédiaire d'un opérateur de compensation, l'article L. 163-2 du Code de l'environnement souligne que le recours au contrat est indispensable. Il dispose, en effet, que « Lorsque des mesures de compensation des atteintes à la biodiversité sont mises en œuvre sur un terrain n'appartenant ni à la personne soumise à l'obligation de mettre en œuvre ces mesures, ni à l'opérateur de compensation qu'elle a désigné, un contrat conclu avec le propriétaire et, le cas échéant, le locataire ou l'exploitant définit la nature des mesures de compensation et leurs modalités de mise en œuvre, ainsi que leur durée ». Possiblement, l'objet de l'obligation principale de ce contrat est alors un (ou des) service(s) environnemental(ux), le propriétaire, le locataire ou l'exploitant s'engageant à le conserver, restaurer, réhabiliter, créer, ou faire évoluer des pratiques.

36. Billet Ph., préc.

37. Alidor B., La compensation et les services écosystémiques, infra.

38. Lucas M., « Le contrat au service de la compensation écologique », EEI, 2017, Dossier 11. 


\section{LE CONTENU DU CONTRAT DE PRESTATION DE SERVICE ENVIRONNEMENTAL}

Qu'elle soit accessoire ou principale, l'obligation environnementale est le plus souvent personnelle (A), néanmoins, la création et le développement de l'obligation réelle environnementale (B) pourrait constituer une réponse appropriée à l'ancrage foncier de nombreux services environnementaux.

\section{A. L'OBLIGATION ENVIRONNEMENTALE PERSONNELLE}

L'obligation environnementale personnelle n'est autre qu'un droit de créance (1) qui présente néanmoins quelques particularités qui rejaillissent sur son exécution (2).

\section{1) La substance de l'obligation environnementale personnelle}

Quel que soit le cadre dans lequel le contrat de prestation de service environnemental est conclu, se pose la question de la nature de l'engagement pris par le débiteur, le plus souvent propriétaire, exploitant ou preneur à bail rural. Ainsi qu'on l'a vu, le débiteur s'engage avant toute chose, et le plus souvent, à réaliser une prestation, terme générique désignant l'objet d'une obligation (art. 1163 C. civ.). Pratiquement, le débiteur s'engage donc à faire quelque chose ${ }^{39}$ : le contrat ou la clause du contrat prescrit alors un comportement ou une abstention. Jusque récemment, cet engagement était désigné dans le Code civil par la qualification d'obligation de faire ou, si le contrat prescrit une abstention, de ne pas faire (ancien art. $1101 \mathrm{C}$. civ.). Bien que ces qualifications ne soient plus envisagées par le Code civil, qui ne vise plus que la « prestation », sans apporter d'autres précisions, il n'en résulte, de notre point de vue, pas de changement : la prestation à la charge du débiteur du service environnemental peut consister tant en une obligation de faire quelque chose que de ne pas le faire. Par exemple, l'obligation d'entretenir les haies et buissons entre le 15 août et le 30 novembre sera qualifiée d'obligation de faire, tandis que l'interdiction de combler les mares et les fossés est une obligation de ne pas faire ; l'une et l'autre constituent une « prestation » au sens de l'article 1163 du Code civil.

39. Comp. Langlais A., préc. p. 197 selon laquelle le service environnemental peut consister en une " chose " si bien que le contrat pourrait alors s'analyser en une vente « ce qui n'est pas sans poser de soucis "». 
Finalement, l'essentiel n'est donc plus là. Il réside davantage dans la création, par le contrat, d'une obligation à la charge du débiteur du service environnemental. Par voie de conséquence, c'est la définition de l'« obligation », pourtant absente du Code civil, qui importe. Une obligation est en effet un lien de droit entre deux personnes, un créancier et un débiteur, en vertu duquel l'une (le créancier) a le droit d'exiger de l'autre (le débiteur) ${ }^{40}$ une prestation. Ainsi définie, il apparaît que l'obligation est donc un engagement personnel : elle conduit à l'exécution d'une prestation par le débiteur ; l'obligation suppose le comportement du débiteur, son action ou son inaction, ainsi que cela a été dit. Le débiteur est donc tenu à exécuter un service environnemental (l'objet de l'obligation principale ou accessoire du contrat, art. 1163 C. civ.) dans le but de préserver ou restaurer un écosystème et donc, in fine, un service écosystémique (le but du contrat, art. 1162 C. civ.). En effet, insistons, le service écosystémique, utilité commune d'un bien, ne peut être l'objet d'une obligation ; il n'est tout au plus que le but du contrat, ou, si l'on préfère, sa cause subjective.

Cette remarque doit conduire à écarter une confusion présente en littérature ; il est, en effet, parfois avancé que le débiteur du service environnemental ne serait tenu qu'à une obligation de moyens et non de résultat au motif que " les cahiers des charges imposent généralement aux agriculteurs une série de pratiques, sans imposer d'en contrôler les résultats environnementaux obtenus (augmentation de la biodiversité, amélioration de la fertilité des sols, etc.). Une obligation de résultats (sic), à l'inverse, consisterait à̀ dédommager les agriculteurs si et seulement si les résultats environnementaux attendus étaient obtenus et vérifiés $»^{41}$. Est alors préconisée une « approche du paiement liée au résultat en termes de production, restauration du service écosystémique » au motif qu'il s'agirait d'une « approche plus directe qui permet de responsabiliser et de professionnaliser les agriculteurs dans leur contribution à la restauration/préservation des services écosystémiques ». Une telle analyse est pourtant trompeuse ; en considérant que le débiteur

40. Poumarède M., Droit des obligations, LGDJ 2014, $\mathrm{n}^{\text {os }} 1$ et $\mathrm{s}$.

41. Duval L., Binet T., Dupraz P., Leplay S., Etrillard C., Pech M., Deniel E., Laustriat M., 2016, Paiements pour services environnementaux et méthodes d'évaluation économique. Enseignements pour les mesures agro-environnementales de la politique agricole commune. Etude réalisée pour le ministère en charge de l'agriculture. Rapport final. Egalement Labous K. et Gruger H, préc. 
devrait être tenu d'une obligation de résultat et que ce résultat est la restauration du service écosystémique, cela revient à le rendre débiteur d'un service écosystémique. Or, 1'on sait que cela ne peut être le cas, le service écosystémique étant une utilité commune d'un bien. Cela ne signifie pas, pour autant, que le débiteur d'un service environnemental serait seulement tenu à une obligation de moyens ${ }^{42}$. Tout au contraire, le service environnemental est le plus souvent une obligation de résultat, qu'il prenne la forme d'une pratique agricole ou d'un système de production agricole. Sans doute, les difficultés à identifier quels services environnementaux exécutés par le débiteur auront une efficacité sur l'écosystème est réelle. Mais, elles n'influent pas sur les services environnementaux auxquels s'engage le débiteur, en ce sens qu'elles n'en modifient pas l'intensité : les liens entre pratiques auxquelles s'engage le débiteur et résultats environnementaux attendus (revalorisation de l'écosystème) doivent être évalués en amont de la conclusion du contrat (ou au cours de son exécution si cela est prévu ainsi), mais ils ne sont pas de nature à influer sur l'intensité des obligations du débiteur qui sont, en ellesmêmes, de résultat ou de moyens. En effet, à l'instar de tous contrats, les négociations précontractuelles auront permis aux parties de définir les objectifs à atteindre et le complexe obligationnel aura été prévu en conséquence, y compris les éventuels contrôles au cours de l'exécution du contrat et sanctions afférentes en cas d'inexécution. En effet, les contrats de prestation de service environnemental sont appelés à durer dans le temps, mais doivent également être adaptables au cours de cette durée.

\section{2) L'exécution de l'obligation environnementale personnelle}

La prestation à la charge du débiteur ne présente, en tant que telle, pas de particularité notable ; ainsi qu'on l'a dit, il s'agit d'une obligation de faire (ou de ne pas faire), le débiteur s'engageant à exécuter un service environnemental. Tenu à titre principal ou accessoire, son obligation sera de résultat ou de moyens, selon ce que les parties auront prévu. Du point de vue contractuel, la particularité est donc ailleurs. Elle est double. 
D'une part, la nécessaire longue durée du contrat doit être combinée avec une certaine souplesse permettant, éventuellement, d'adapter le service dû par le débiteur au cours de l'exécution du contrat.

Il est certain que l'obtention de résultat environnementaux (les services écosystémiques), cause subjective de la clause environnementale, suppose une durée certaine : les obligations doivent être pérennes. Le Code civil offre désormais un cadre plus sûr aux contrats de durée (art. 1210 et ss. C. civ.), tels les contrats de prestation de service environnemental. En effet, par hypothèse un tel service est tantôt répété dans le temps, tantôt maintenu dans la durée, ce qui suppose, par ailleurs, que son exécution soit contrôlée. Ainsi sera évaluée, sur la base d'un état des lieux initial (faune, flore, etc.), la correcte exécution du service environnemental dans les termes du contrat : ce contrôle pourra être visuel (de la part du créancier), basé sur l'enregistrement des pratiques agricoles par le débiteur (tableau de suivi, etc.), fondé sur un suivi scientifique par un tiers, etc.

Mais, les difficultés réelles à corréler les services environnementaux avec la plus-value environnementale - le maintien ou le développement de l'écosystème et de ses services - recherchée par le créancier impliquent une certaine souplesse ${ }^{43}$. L'adaptation de l'engagement aux dynamiques environnementales pourra s'obtenir de différentes manières : ici, le contrat sera conclu pour une relative courte durée, mais prévoira les modalités de son renouvellement. Là, une période «d'essai » sera prévue, permettant de tester des pratiques agricoles afin d'engager le débiteur dans des obligations pérennes. Encore, l'on prévoira des clauses d'adaptation permettant, dans une certaine mesure, de faire évoluer les services environnementaux afin de les adapter aux résultats recherchés dans un souci d'efficacité.

Néanmoins, ces processus contractuels pouvant être mis en œuvre pour assurer la pérennité du service environnemental, seront de peu d'utilité en l'absence d'ancrage territorial du contrat.

Telle, d'autre part, est la seconde particularité des contrats de prestation de service environnemental : si le service environnemental peut être lié à la personne, le débiteur, il peut l'être davantage encore au fonds. En effet, souvent, le service environnemental ne présente

43. Lucas M., « Le contrat au service de la compensation écologique », préc. 
d'intérêt que s'il est territorialisé : il suppose alors un ancrage foncier ${ }^{44}$ en lien avec la durée : le cycle de production d'une futaie de chênes peut dépasser le siècle ! L'attachement au fonds du service environnemental devient donc souvent une question primordiale afin d'assurer non seulement la pérennité mais également la stabilité des services environnementaux. Si cela pose peu de difficultés lorsque la clause environnementale est accessoire dès lors qu'elle suivra la durée du contrat principal (par exemple à un bail rural), elles s'accroissent lorsque le service environnemental est l'objet d'une obligation personnelle principale : attaché à la personne du débiteur, il ne l'est pas au fonds. Concrètement, en cas de cession du terrain, de disparition du débiteur (décès, liquidation), (etc.) le contrat de prestation de service environnemental pourrait perdre toute efficacité, voire s'éteindre s'il n'a pas été prévu une transmission des obligations aux ayant-causes à titre particulier, voire universels. Telle est la raison pour laquelle, d'aucuns cherchent à mettre en avant la constitution de droits réels au service de l'environnement : il s'agit alors d'attacher le service environnemental à la propriété et non pas seulement à la personne en créant une charge réelle affectant un fonds et comme telle opposable à ses propriétaires successifs. Bien que la plupart des conventions prévoient encore, semble-t-il, des obligations personnelles à la charge d'un débiteur (propriétaire, preneur, exploitant, etc.), plusieurs pistes imparfaites mais cherchant à mettre en œuvre une valorisation durable et stable ont, ainsi, été explorées, dont certaines ont pu être retenues par la doctrine, la pratique ou encore la jurisprudence. C'est ainsi qu'en marge du contrat de prestation de service environnemental ont été imaginés, et parfois utilisés, d'autres contrats : contrats constitutifs d'un droit d'usage voire d'un usufruit, servitudes conventionnelles environnementales ${ }^{45}$, constitution d'un droit réel de jouissance spéciale $^{46}$, bail emphytéotique, constitution d'une fiducie foncière

44. Lucas M., « Le contrat au service de la compensation écologique », préc.

45. Sur ses limites, v. Boutonnet M, «Servitude environnementale conventionnelle ou contrat constitutif d'obligation réelle environnementale », in Boutonnet M. (dir.), Le contratetl'environnement, Etude de droitinterne, international et européen, PUAM, 2014, p. 271 ; Sarlat J.-J. et Olivier A., « La servitude conventionnelle environnementale », Env. et DD 2011, Etude 7.

46. Civ. $3^{\text {e }}, 31$ oct. 2012 , n ${ }^{\circ} 11-16.304$, D. 2012. 2596, obs. Tadros A. ; D. 2013. 53, note d'Avout L. et Mallet-Bricout B., et 2123, obs. Reboul-Maupin N. ; AJDI 2013. 540, obs. Cohet-Cordey F. ; RDI 2013. 80, obs. Bergel J.-L. ; RTD civ. 2013. 141, obs. Dross W. ; Civ. $3^{\text {e }}, 28$ janv. 2015, n 14-10.013, D. 2015. 599, note Mallet-Bricout B., 988, chron. Méano A.-L., et 1863, obs. Reboul-Maupin N. ; AJDI 2015. 304, obs. Le Rudulier N. ; RDI 2015. 175, obs. Bergel J.-L. ; RTD civ. 2015. 413, obs. Dross W., et 619, obs. Barbier H. ; 
environnementale ${ }^{47}$. Dans le même temps est néanmoins née l'obligation réelle environnementale. Cette dernière, consacrée par la loi n ${ }^{\circ} 2016-1087$ du 8 août 2016, pourrait être, sous conditions, l'obligation principale d'un contrat de prestation de service environnemental.

\section{B. L'OBLIGATION RÉELLE ENVIRONNEMENTALE}

L'obligation réelle environnementale. L'obligation réelle environnementale a fait son apparition dans l'article L. 132-3 du Code de l'environnement ${ }^{48}$ en vertu duquel « Les propriétaires de biens immobiliers peuvent conclure un contrat avec une collectivité publique, un établissement public ou une personne morale de droit privé agissant pour la protection de l'environnement en vue de faire naître à leur charge, ainsi qu'à la charge des propriétaires ultérieurs du bien, les obligations réelles que bon leur semble, dès lors que de telles obligations ont pour finalité le maintien, la conservation, la gestion ou la restauration d'éléments de la biodiversité ou de fonctions écologiques. " Si les contours d'un tel contrat ne sont pas encore déterminés avec certitude, faute que la notion et le régime de l'obligation réelle environnementale soient réellement connus, rien ne semble s'opposer à ce que cette obligation réelle ait pour objet un service environnemental, même si les conditions très strictes (formalisme, qualité des parties, finalités, etc.) et les précautions prises (droits du preneur, droits des tiers) par les textes ne permettent pas sa généralisation y compris dans le champ de la compensation ${ }^{49}$. Quoi qu'il en soit, l'on aura compris que le mécanisme ainsi institué permet d'une part d'obliger un débiteur, personne physique ou morale, à des obligations de faire ou de ne pas faire, et d'autre part d'attacher ces obligations, en quelque sorte, au fonds, ce qui accroit leur pérennité et leur stabilité. En effet, pour une durée qui pourrait aller jusqu'à 99 ans, les propriétaires successifs du fonds, ayants cause à titre particulier, seront tenus aux obligations de faire et de ne pas faire prévues au contrat de prestation de service

JCP 2015. 148, veille Milleville S. ; JCP N 2015. 1083, note Dubarry J. et Julienne M. ; JCP 2015. 252 note Revet T.

47. V. Droits réels au profit de la biodiversité : comment le droit peut-il contribuer à la mise en cuvre des paiements pour services environnementaux? Rapport de la mission économie de la biodiversité, Fondation Nicolas Hulot pour la nature et l'homme, 2013.

48. Martin G. J., « Les potentialités de l'obligation réelle environnementale », Dr. Env., oct. 2016 , p. 334 et s.

49. Sur les potentialités en matière de compensation, v. néanmoins, Martin G.-J., préc. 
environnemental (et publiées au fichier immobilier) : obligation propter rem, l'obligation réelle environnementale pèse en effet sur le propriétaire du fonds qui, es qualité, devra donc exécuter le service environnemental.

En conclusion, le contrat échoue, en première intention, à saisir les services écosystémiques : utilités communes d'un bien, ils ne peuvent comme tels être l'objet d'une obligation contractuelle dont le propriétaire d'un fonds ou son exploitant pourraient être débiteurs. Ce faisant, cette analyse, qui rejoint l'idée ici mise en avant que le propriétaire (ou l'exploitant) ne coproduit pas les services écosystémiques, laisse ces services hors du commerce.

Mais, le contrat peut, tout de même, les favoriser : il s'agit alors de contractualiser les services que l'Homme peut rendre à la nature, les " services environnementaux », qui prendront, notamment, la forme de pratiques agricoles ou de système de production agricoles plus favorables à l'environnement que ne le sont le strict respect des lois et règlements et l'application des bonnes pratiques agricoles.

Objet d'une obligation principale ou accessoire, les services environnementaux tendent alors à favoriser les écosystèmes et, par voie de conséquence, les services écosystémiques qui apparaissent tels le « but» des contrats de prestation de service environnemental. 



\title{
Compensation et services écosystémiques
}

\author{
Bastien ALIDOR \\ Doctorant, Université Toulouse Capitole \\ IEJUC, F-31000
}

La protection des sols et des services écosystémiques qu'ils produisent s'est récemment enrichie du mécanisme, connu et renforcé, de la compensation. Toutefois, une brève incursion dans les perspectives offertes par l'utilisation de l'outil contractuel nous permettra d'en nuancer l'apport.

Le mécanisme est connu car il n'est pas nouveau. Depuis les lois du 10 juillet 1976 et du 2 février 1995 (dite « loi Barnier ») la compensation est présentée au sein de l'étude d'impact, première forme d'une prise en compte des impacts de l'activité humaine ex ante ${ }^{2}$. Elle est ensuite déclinée sous plusieurs formes dans différents secteurs du droit de l'environnement : en matière de défrichement (art. L. 341-6 C. for.) ou d'atteinte aux objectifs de conservation d'un site Natura 2000 (art. L. 414-4 VII C. env.).

Le mécanisme est renforcé par la loi no 2016-1087 du 8 août 2016 pour la reconquête de la biodiversité, de la nature et des paysages,

\footnotetext{
1. Loi $\mathrm{n}^{\circ} 76-629$ du 10 juillet 1976 relative à la protection de la nature et loi $\mathrm{n}^{\circ} 95-101 \mathrm{du}$ 2 février 1995 relative au renforcement de la protection de l'environnement. L'article 2, $4^{\circ}$ du décret d'application $n^{\circ} 77-1141$ du 12 octobre 1977 prévoyait que l'étude d'impact devait présenter « les mesures envisagées par le maître de l'ouvrage ou le pétitionnaire pour supprimer, réduire et si possible compenser les conséquences dommageables du projet sur l'environnement».

2. Au niveau international, le premier texte à prévoir la compensation est la Convention de Ramsar sur les zones humides internationales du 2 février 1971 qui prescrit de « compenser autant que possible toutes pertes de ressources en zones humides » en cas de retrait d'une zone humide inscrite sur la liste des sites Ramsar (art. 4.2).
} 
dite loi « Biodiversité $»^{3}$, qui consacre la compensation à l'article L. 110-1 du Code de l'environnement en s'affranchissant d'une logique sectorielle jusque-là dominante ${ }^{4}$ et en entrant dans les principes généraux du droit de l'environnement. Le principe d'action préventive, qui fait partie de ces derniers, est désormais complété (art. L110-1-II $2^{\circ}$ in fine C. env.) par l'intégration de la séquence ERC (éviter, réduire, compenser) prévoyant qu' " en dernier lieu », si l'évitement ou la réduction ne sont pas possible, il faudra « compenser les atteintes (...) en tenant compte des espèces, des habitats naturels et des fonctions écologiques affectées; ce principe doit viser un objectif d'absence de perte nette de biodiversité, voire tendre vers un gain de biodiversité ». Cette compensation s'opère

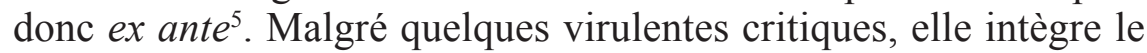
principe de prévention ${ }^{6}$ : ce point est important car il révèle aussi la dimension exceptionnelle de la mesure de compensation qui n'est que subsidiaire à la mesure de réduction et que très subsidiaire à celle d'évitement ${ }^{7}$. Si le couplage entre prévention et compensation au sein des principes généraux ne suffisait pas, le second alinéa de l'article L. 161-1-I du Code de l'environnement précise bien que les mesures de compensation « ne peuvent pas se substituer aux mesures d'évitement et de réduction ».

3. Van Lang A., « La loi Biodiversité du 8 août 2016 : une ambivalence assumée », AJDA 2016, p. 2381.

4. Pour le dépassement d'une logique sectorielle concernant les services écosystémiques Cf. supra Doussan I., « Brève histoire de l'intégration de la notion de service écosystémique en droit».

5. V. Combe M., « Le régime juridique de l'obligation de compensation écologique », EEI, $\mathrm{n}^{\circ} 6$, Juin 2017, dossier $8:$ : (...) la compensation ex ante désigne l'ensemble des actions mises en œuvre pour contrebalancer les dommages prévus - mais non encore réalisés - par un projet, plan ou programme sur l'environnement qui n'ont pas pu être suffisamment évités ou réduits ».

6. Contra Lucas M., 2015, Étude juridique de la compensation écologique, LGDJ, T. 11 : pour l'auteur la compensation ne procède pas de la prévention, voy. spéc. p. $67 \mathrm{n}^{\circ} 77$ : les mesures de compensation " ne visent pas à empêcher la réalisation du dommage environnemental. Elles ont plutôt pour but de pallier les effets préjudiciables d'un projet ou d'un plan sur l'environnement. Elles n'agissent pas sur la cause mais sur les effets (...). Dans le meilleur des cas, ces mesures seront localisées à proximité immédiate du site endommagé ».

7. Pour l'importance de l'évaluation dans la prévention voy. Jégouzo Y., « De certaines obligations environnementales : prévention, précaution et responsabilité », AJDA 2005, p. 1164 ; adde Steichen P., «Le principe de compensation : un nouveau principe du droit de l'environnement? », in C. Cans (dir.), La responsabilité environnementale. Prévention, imputation, réparation, coll. «Thèmes et commentaires », Dalloz 2009, p. 143. Pour une critique de l'objectif d' " absence de perte nette » voy. Billet Ph., « De la relativité de la neutralité environnementale en matière de compensation écologique », $E E I, \mathrm{n}^{\circ} 6$, Juin 2017, dossier 10 . 
Concernant le cadre général de la loi nouvelle, le vœu formulé par le ministre de l'environnement au moment où il présenta le projet de loi relatif à la biodiversité était de consacrer un dispositif « à la biodiversité, prise dans son ensemble : depuis les gènes jusqu'au niveau le plus important d'organisation des écosystèmes que sont les paysages, sur terre comme en mer $\gg{ }^{8}$. Ce souhait exprime une ambition particulièrement forte qui s'est traduite par la consécration de quelques innovations majeures que nous ne développerons pas dans cette contribution mais qu'il convient de souligner (la loi Biodiversité est porteuse de nombreuses innovations qui modifie très largement le paysage juridique ${ }^{9}$ ). Nous retiendrons que la loi nouvelle prête désormais à la biodiversité une "valeur d'usage " (le terme est employé dans le texte de la loi) et une valeur d'échange qui la fait entrer pleinement dans le giron du droit privé des contrats, véritables vecteurs des échanges économiques ${ }^{10}$. L'exposé des motifs du projet de loi souligne, à ce propos, le fait que « la biodiversité est une force économique pour la France » puisqu'elle assure des « services écosystémiques » dont le « coût de (la) disparition » ne peut pas encore être connu ${ }^{11}$. L'esprit de la loi est donc de laisser aux acteurs économiques, qui n'auront pu ni éviter, ni réduire leur impact sur la biodiversité, une possibilité de produire un équilibre entre atteintes et mesures compensatoires pour tendre vers une sorte de « neutralité $\rangle^{12}$, voire 《 d'additionnalité $»^{13}$ écologique. À ce titre, l'usage du contrat comme vecteur de protection ou de compensation

\footnotetext{
8. Projet de loi n ${ }^{\circ} 1847$ du 26 mars 2014 et relatif à la biodiversité, présenté par Ph. Martin. 9. Pour ce qui est de notre matière c'est la création de l'Agence française pour la Biodiversité (établissement public administratif, elle jouera un rôle incontournable dans la mise en œuvre de la compensation) et la consécration du préjudice écologique qu'il nous faut souligner; pour des précisions sur les apports de la loi voy. Ch. Cans, O. Cizel, Loi Biodiversité, ce qui change en pratique, Éditions Législatives, 2017, spéc. p. 62 et ss..

10. V. supra Poumarède M., "Services écosystémiques et contrat. Quelle obligation contractuelle environnementale ?».

11. Pour un approfondissement de la question économique voy. Faure M., L'analyse économique du droit de l'environnement, Bruylant, 2007, préf. M. Prieur.

12. Billet $\mathrm{Ph} .$, « La «neutralité environnementale » : esquisses juridiques », in Mélanges en l'honneur de François Collart-Dutilleul, Dalloz, 2017, p. 103 et s. ; l'auteur y identifie trois sortes de neutralités :

- la neutralité par évitement traduisant une véritable action préventive par priorité à la source

- la neutralité par compensation permettant le rééquilibrage des atteintes inévitables

- la neutralité par réparation reposant sur une remise en état des lieux affectés.

13. Voy. Lucas M., Étude juridique de la compensation écologique, précité, p. 358, nº 430 et $\mathrm{s}$. : « Distinct du critère d'équivalence, ce mécanisme exige schématiquement que la mesure compensatoire crée un « plus » pour contrebalancer un « moins » correspondant aux impacts de l'opération envisagée, de la planification ou du dommage effectif causé ».
} 
n'est pas nouveau et celui-ci s'est développé de manière éparse. Dans la matière, chaque domaine bénéficie d'une protection particulière : le droit de l'eau connait des contrats de rivière ${ }^{14}$, le droit rural met en avant ses baux incluant une clause environnementale ${ }^{15}$, le droit des installations classées va de sa spécificité en consacrant en matière de vente immobilière une obligation d'information ${ }^{16}$ ou en usant des clauses de passif environnemental. Tant et si bien qu'il est devenu difficile d'être exhaustif dans l'énumération des figures contractuelles susceptibles d'intégrer dans leurs prévisions une préoccupation environnementale. La « contractualisation du droit de l'environnement » ne fait cependant plus aucun doute ${ }^{17}$.

Dans le cadre de la compensation, le maître d'ouvrage pourra s'acquitter de son obligation de plusieurs manières. Trois d'entre elles sollicitent le contrat comme mode d'organisation des mesures de compensation. Le premier type de contrat vise la collaboration avec l' « opérateur de compensation », personne publique ou privée chargée de mettre en œuvre les mesures et de les coordonner sur le long terme (art. L. 163-1-III, C. env., il s'agirait là d'un contrat d'entreprise au sens civiliste). Le deuxième fait référence à l'acquisition d'unités de compensation qui suggère une vision dématérialisée ${ }^{18}$ de la compensation (contrat de vente). En effet, il s'agit dans ce cas, d'autoriser le maître d'ouvrage à acheter des unités produites sur des "sites naturels de compensation ${ }^{19}{ }^{19}$ (art. L. 163-3 C. env.). La dernière est la possibilité laissée au propriétaire par l'article L. 132-3 du Code de l'environnement de créer contractuellement une obligation réelle environnementale ayant pour finalité le maintien, la conservation, la gestion ou la restauration d'éléments de la biodiversité.

14. Utilisés dans la mise en œuvre des schémas d'aménagement et de gestion des eaux (SAGE).

15. V. infra Krajeski D., « Travail du sol, services écosystémiques et bail rural »; Etrillard C., « La compensation écologique : une opportunité pour les agriculteurs », Dr. rur. n 441, Mars 2016, étude 10.

16. Art. L514-20 C. env. ; voy. Asscher J., « L'article L514-20 du Code de l'environnement : une obligation d'information tronquée », Gaz. Pal. nov-déc. 2007, p. 3941.

17. Voy. Monteillet V., 2017, La contractualisation du droit de l'environnement, préf. Pélissier A., Dalloz, Nouvelle Bibl. de Thèses, vol. 168.

18. Martin G.-J., « Les unités de compensation dans la loi n $2016-1087$ du 8 août 2016 pour la reconquête de la biodiversité, de la nature et des paysages ", Droit et Ville, 2017, à paraître.

19. La terminologie a évolué au gré des travaux parlementaires et la formule a été préférée à celle de « réserve d'actifs naturels », plus prompte à engendrer une polémique. 
La flexibilité du contrat permet d'apporter à la prescription administrative de compensation une réponse cohérente, en adéquation avec les qualités pédologiques des milieux subissant une perte de biodiversité. Il n'en reste pas moins une limite de taille tenant à la pérennisation des mesures. La compensation in situ ne sera pas toujours possible et la localisation des mesures sera alors tributaire de la disponibilité du foncier. Marthe Lucas a souligné les conséquences probables de cet éparpillement des sites de compensation : " augmentation des effets de lisière, perte de cohérence écologique, difficulté d'en contrôler et d'en évaluer les résultats globaux, accroissement du nombre de partenaires et de contrats $\gg{ }^{20}$.

Dans ce contexte, l'approche plutôt économique de la compensation qui vise à remplacer une perte par un gain laisse au juriste une impression de trompe-l'œil. Le dispositif proposé protège-t-il efficacement le sol et les services qu'ils fournissent à la société ? Plus largement, protège-t-il le patrimoine naturel qui « génère des services écosystémiques et des valeurs d'usage » (nouvel art. L. 110-1-I C. env.) ? Posées ainsi, ces questions appellent la remarque suivante : l'atteinte à un écosystème ou aux fonctions écologiques n'est-elle pas indissociable des services rendus aux activités humaines ?21 La compensation ex ante ne fait pourtant pas référence à ces derniers. Si tel est bien le cas on assisterait subrepticement à un renversement de paradigme : la vision purement économique attachée à la notion de services écosystémiques céderait devant l'impérieuse nécessité de préserver le milieu naturel, sans prendre en compte les intérêts des personnes humaines. Il n'en demeure pas moins que les articles de la loi ayant trait à la compensation conserve un champ lexical symptomatique d'une approche comptable qu'on a du mal à détacher d'une vision toujours plus anthropocentrée du droit de l'environnement. Dès lors, il devient inévitable de se demander si la notion de services écosystémiques peut apporter quelque chose aux différentes voies de la compensation.

C'est d'abord celles-ci que nous tâcherons d'étudier en rappelant quels sont les nouveaux mécanismes de la compensation et le contrôle auquel ils sont soumis (I). Puis, la loi nouvelle consacrant la

20. Lucas M., « Le contrat au service de la compensation écologique », EEI n 6, Juin 2017, dossier 11.

21. Pour un panorama des services écologiques voy. : http://uicn.fr/wp-content/ uploads/2016/09/Brochure_Panorama_des_services-vol1.pdf 
notion de « services écosystémiques », il conviendra de la confronter au prisme de la compensation afin de savoir si elle agit sur le mécanisme comme une sorte de catalyseur ou si, au contraire, elle entrave sa bonne réalisation (II).

\section{LA COMPENSATION DANS LA LOI BIODIVERSITÉ}

La compensation implique une « sécurisation du foncier » (c'est le vocabulaire utilisé par les acteurs de la compensation ${ }^{22}$ ). Autrement exprimé, ce qui posera problème c'est l'utilisation de nouveau sols disponibles pour mettre en œuvre la compensation. Cette disponibilité n'étant pas assurée, il a été convenu de consacrer plusieurs moyens visant à compenser. Nous verrons donc d'abord les différents mécanismes (A) avant de voir comment la compensation est contrôlée (B).

\section{A. LES MÉCANISMES DE LA COMPENSATION}

Le maître d'ouvrage soumis à l'obligation légale peut prendre en charge « directement» (art. L. 163-1-II C. env.) la mesure de compensation. Néanmoins, s'il ne souhaite pas procéder lui-même à la compensation, il aura une option et pourra utiliser deux types de contrats soit en faisant appel à un «opérateur de compensation $»^{23}$ (art. L. 163-1 III C. env.), soit par « acquisition d'unités de compensation » (art. L. 161-3 C. env.). Ces deux mécanismes sont complétés par l'obligation réelle environnementale qui peut également être un support juridique de compensation ${ }^{24}$.

22. V. par exemple le site internet de la CDC Biodiversité : http://www.cdc-biodiversite.fr/ nos-metiers/compensation-ecologique/

23. Le projet de loi présenté en première lecture devant le Sénat soumettait la qualité d'opérateur à l'agrément de l'autorité administrative (Sénat n ${ }^{\circ} 69,26$ janvier 2016) et son rapport était intéressant : "Compte tenu des enjeux de la compensation, de la technicité des mesures à mettre en œuvre et de leur durée, il est nécessaire d'encadrer cette activité particulière. L'agrément doit notamment permettre de garantir que l'opérateur possède l'expertise technique, les capacités financières et l'indépendance nécessaire, pour mettre en œuvre les mesures de compensation pour le compte d'une personne soumise à une telle obligation. Un tel agrément sécurise la mise en œuvre de la compensation, et complète l'agrément prévu pour les réserves d'actifs naturels ».

24. Sur la nature des contrats (droit public/droit privé) voy. Monteillet V., La contractualisation du droit de l'environnement, précité, p. 85, n 109 et ss.. 
Dans le premier cas, où le débiteur légal de la compensation (le maître d'ouvrage) contracte avec un opérateur, la relation contractuelle est inopposable à l'autorité administrative. Ce qui signifie très concrètement que seul le maître d'ouvrage sera responsable aux yeux de l'autorité administrative. L'opérateur pourra mettre en œuvre la mesure de compensation soit sur un terrain lui appartenant, soit sur le terrain d'un tiers ${ }^{25}$. Il n'est que précisé qu'il s'agit de «personnes morales ou personnes privées ». Il est d'ailleurs étonnant de relever que les travaux parlementaires avaient un temps envisagé de soumettre la qualité d'opérateur à l'agrément avant d'abandonner l'idée. Le manque de précision quant au statut de ce nouvel acteur de la biodiversité questionne d'autant plus qu'il est censé mener la compensation pour le compte du maître d'ouvrage qui restera, seul, tenu responsable de sa réalisation.

Dans le deuxième cas, l'achat d'unités de compensation fait nécessairement penser à une dématérialisation de la protection des sols. L'idée générale est d'éviter une " perte nette » de biodiversité en palliant la mise en œuvre d'une compensation techniquement insurmontable en produisant des unités de compensation. C'est ce qui permet au législateur de consacrer une obligation de compensation de résultat. Il importe peu que la compensation par la demande ${ }^{26}$ soit impossible, l'aménageur pourra toujours envisager l'acquisition d'unités (compensation par l'offre ${ }^{27}$ ) : l'intensité de l'obligation visée par la loi doit donc nécessairement être celle relative à la mise en œuvre de l'opération de compensation. Si l'obligation de résultat visait l'efficacité de la mesure (ce que pourrait laisser faussement penser le texte), il deviendrait logiquement impossible de vendre des unités issues de sites dont les résultats écologiques ne sont pas encore atteints... Pour encadrer ce marché émergent le législateur a

25. Cette perspective se heurte à ce que les opérateurs nomment « la maîtrise du foncier » ou la « sécurisation du foncier » : de façon prépondérante ils ne sont pas propriétaires des terres sur lesquelles seront mise en œuvre la mesure et feront appel à des tiers (l'obligation réelle environnementale pourra dans ce cas être utilisée, $c f$. infra).

26. V. la définition donnée par Leray G., « La responsabilité environnementale de l'entreprise à l'épreuve de la compensation ", $R L D A \mathrm{n}^{\circ} 128,1^{\text {er }}$ juillet $2017:$ « (...) un opérateur de compensation sécurise, conformément à un contrat, des terrains pour le compte d'un débiteur à une obligation de compensation ».

27. Ibid. : « (...) un opérateur de compensation acquiert des terrains qu'il aménage en « sites naturels de compensation ». De la division fictive de leurs utilités environnementales sont tirées des " unités de compensation ", ensuite proposées à des maîtres d'ouvrage soumis à une obligation de compensation environnementale ». 
soumis les sites naturels de compensation à agrément ${ }^{28}$; parmi les critères d'obtention, la personne qui met en œuvre la mesure doit avoir les " capacités techniques et financières nécessaires (...) ", ce qui laisse à l'autorité administrative une marge d'appréciation suffisante pour juger de la qualité des opérateurs autant que de celle des sites sur lesquels seront effectuées les mesures.

Enfin, la compensation peut aussi être mise en œuvre par le jeu de la nouvelle obligation réelle environnementale ${ }^{29}$. Il s'agit ici de donner au propriétaire d'un immeuble ${ }^{30}$ la possibilité de grever son fonds d'une " obligation environnementale intuiti rei durable et automatiquement transmissible à ses ayants cause $\rangle^{31}$. Selon les termes d'une première version du projet de loi cet enjeu répondait à un double objectif : " faciliter le développement d'actions pérennes permettant de stopper l'érosion de la biodiversité ", d'une part « permettre à un propriétaire de mettre en place simplement sur sa propriété une démarche contractuelle $\|^{32}$, d'autre part. Il fallait trouver, par le contrat, une solution pérenne à la protection du sol objet de propriété. Or, l'obligation réelle est « un outil proposé à un propriétaire privé qui souhaite promouvoir une gestion de son bien conforme à la protection de la nature, en faisant usage de la liberté contractuelle pour adapter les obligations consenties à l'objectif qu'il poursuit, en tenant compte des spécificités du site et des besoins particuliers de protection $\|^{33}$. Aux termes du nouvel article L. 132-3 du Code de l'environnement, « les propriétaires de biens immobiliers peuvent conclure un contrat avec un collectivité publique, un établissement public ou une personne morale de droit privé agissant pour la protection de l'environnement en vue de faire naître à leur charge, ainsi qu'à la charge des propriétaires ultérieurs du bien, les obligations réelles que bon leur semble, dès lors que de telles obligations ont pour finalité le maintien, la conservation,

28. Décret $n^{\circ}$ 2017-265 du 28 février 2017 relatif à l'agrément des sites naturels de compensation.

29. Herrnberger O., «L'obligation réelle environnementale, le point de vue de la pratique », $E E I \mathrm{n}^{\circ}$ 6, Juin 2017, dossier 17.

30. Le terme a une signification particulière en droit civil puisqu'il correspond principalement aux « fonds de terre » et aux « bâtiments » réputés « immeuble par nature » par l'article 518 du Code civil.

31. V. supra Beaussonie G., «La qualification juridique des services écosystémiques ».

32. Ass. Nat. $n^{\circ} 1847,26$ mars 2014, projet de loi relatif à la biodiversité.

33. Martin G.-J., « Les potentialités de l'obligation réelle environnementale », Dr. Env. $\mathrm{n}^{\circ} 249,2016$, p. 334 , spéc. p. 335. 
la gestion ou la restauration d'éléments de la biodiversité ou de fonctions écologiques ». Le mécanisme, connu à l'étranger ${ }^{34}$, n'est pas sans rappeler celui de la servitude, ce qui a fait naître et ce qui nourrira certainement un débat récurrent sur la nature juridique du dispositif, à cheval entre le droit des biens et le droit des contrats ${ }^{35}$. Il ne s'agit ici ni d'une obligation personnelle à l'instar du bail environnemental ${ }^{36}$, ni d'une servitude qui suppose un rapport entre fonds dominant et fonds servant ${ }^{37}$ (du moins concernant la servitude de droit privé). Le mécanisme correspond de manière plus générale à une obligation propter rem $^{38}$ ayant le double avantage d'offrir à la fois une certaine liberté dans l'établissement de son contenu (en réalité on parlera bien de liberté contractuelle, désormais prévue par l'article 1102 du Code civil) et en restant opposable erga omnes puisqu'elle n'est pas liée à une personne mais à un fonds. C'est dans cette philosophie que le Professeur G.-J. Martin souhaitait inscrire l'obligation réelle en laissant aux opérateurs « un outil marqué par la souplesse, la liberté contractuelle, l'autonomie de la volonté ${ }^{39}$. Le contrat abritant l'obligation réelle prendra la forme

34. V. Mekki M., «Les conservation easements en droit américain », in Le contrat et l'environnement, Étude de droit comparé, M. Hautereau-Boutonnet (dir.), Bruylant, 2015, p. 115, spéc. p. 123 pour la définition : « (...) droit réel sur un bien foncier qui impose des limites ou des obligation expresses, dont les objectifs comprennent la rétention ou la protection des valeurs naturelles, panoramiques ou des espaces ouverts de la propriété ; l'assurance de sa disponibilité à des fins d'utilisation agricole, forestière, récréative ou d'espace ouvert ; la protection des ressources naturelles, le maintien ou l'amélioration de la qualité de l'air ou de l'eau ainsi que la protection de l'aspect historique, architectural, archéologique ou culturel de la propriété ».

35. V. Denizot A., «L'obligation réelle environnementale ou droit réel de conservation environnementale ? Brève comparaison franco-chilienne de deux lois estivales ", RTD civ. 2016, p. 949.

36. Le preneur à bail rural peut ajouter à ses obligations traditionnelles des engagements d'ordre écologique, soit dans le même contrat (c'est le bail environnemental prévu à l'article L. 411-27 du Code de l'environnement), soit par acte séparé envers une tierce personne (mesures agri-environnementales souscrites auprès de l'État).

37. Art. 686 C. civ. ; pour une opinion plus nuancée concernant le rapport entre fonds

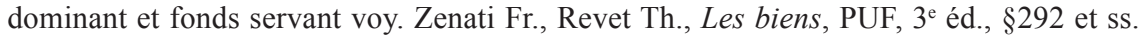
(spéc. p. 461).

38. Celle adjointe au droit réel démembré ; pour un auteur il s'agit de l'« accessoire du droit réel » devant « être cantonné à permettre à son créancier de tirer un meilleur profit des utilités qui lui sont réservées » (cf. DROSS W., Droit des biens, Lextenso, Domat, $2^{\mathrm{e}}$ éd., $\mathrm{n}^{\circ} 131$ ) ; une partie de la doctrine y voit cependant l'obligation réelle principale ; voy. par ex. A. TADROS, «La réhabilitation des droits réels in faciendo : réjouissance ou maladresse ? », RDC 2015, $\mathrm{n}^{\circ} 4$ Contra Dross W., «L'obligation réelle peut-elle se concevoir indépendamment du service d'un droit réel démembré ? », RTD civ. 2014, p. 684. 39. Martin, G. J. " La servitude environnementale de droit privé », in Les servitudes environnementales, Huten N., Struillou J.-F. (dir.), Cah. GRIDAUH 2015, n 28, p. 61 ; 
d'un acte authentique dont le contenu sera déterminé par les parties. Le propriétaire peut ainsi affecter une utilité de l'immeuble à une fin écologique. Il pourra le faire pour préserver la richesse écologique de son sol ou pour valoriser une ressource, mais la démarche tient de l'altruisme écologique puisqu'aucune réelle incitation n'est prévue par le texte. Cette voie ne sera empruntée que lorsque les personnes privées seront assurées de pouvoir en retirer une satisfaction particulière liée à leur désir de protéger une ou plusieurs ressources de leurs sols. En revanche, l'obligation réelle peut également servir à redonner au débiteur d'une mesure compensation (art. L. 132-3 al. 2 C. env.) une emprise sur sa « dette de compensation ». Cela de deux manières différentes. Dans un premier cas, l'aménageur qui fait appel à un opérateur reste seul tenu pour responsable face à l'administration de la bonne exécution de la mesure de compensation. En grevant son fonds d'une obligation réelle il permet de faire entrer dans la relation ce que d'aucuns ont appelé « un tiers garant $»^{40}$ qui veillera, à la place du maître d'ouvrage, à la bonne exécution de la mesure. Dans un second cas, le propriétaire peut produire lui-même des unités de compensation en cherchant à obtenir l'agrément pour le fonds qu'il aura grevé d'une obligation réelle. Dans ce cas, le propriétaire foncier, ou l'exploitant s'il donne son accord, pourront se positionner sur le marché de la compensation en offrant des unités de biodiversité. Ce modèle de la compensation par l'offre est bien connu aux Etats-Unis ${ }^{41}$ et soulèvera immanquablement la question du contrôle et de l'encadrement réglementaire ${ }^{42}$.

du même auteur : «Pour l'introduction en droit français d'une servitude conventionnelle ou d'une obligation propter rem de protection de l'environnement », RJE 2008, p. 123 ; «L'obligation réelle environnementale : un objet juridique non identifié ? », Annales des Loyers, Avril 2017, p. 123.

40. Doussan I., « Compensation écologique : le droit des biens aux services de la création de valeurs écologiques et après ? ", in Repenser la propriété, un essai de politique écologique, Guibet-Lafaye C., Vanuxem S. (dir.), PUAM, 2015, p. 99, spéc. pp. 108 et 109.

41. Il s'agit des Mitigations Banks et des Conservation Banks qui sont des institutions rassemblant l'ensemble des crédits de compensation détenus par des acteurs privés ou des États, afin de les revendre dans le cadre de futurs projets d'aménagement. Voy. Géniaux G., «Le Mitigation Banking : un mécanisme décentralisé au service des politiques de « No net loss », in Les difficultés de mise en œuvre de la Directive Habitat 2000, INRA, n 19, 2002, p. 57.

42. À ce titre, les premières versions du projet de loi Biodiversité prévoyaient l'agrément administratif des opérateurs de compensation. Celui-ci a été abandonné au cours des travaux parlementaires, délaissant ainsi une forme de contrôle. 
Ces mêmes perspectives se retrouvent dans l'élaboration de droits réels de jouissance spéciale, assez récemment consacrés par la Cour de cassation ${ }^{43}$. Cette consécration avait pour objectif de laisser au propriétaire la possibilité de consentir à un tiers le bénéfice d'un droit réel sur son bien lui permettant une jouissance spéciale ${ }^{44}$. Contrairement à ces derniers la loi de 2016 a néanmoins prévu deux formes d'incitation concernant l'obligation réelle : d'une part, le contrat mettant en place une telle obligation est dispensée de droit d'enregistrement et de taxe de publicité foncière ${ }^{45}$; d'autre part, depuis le $1^{\text {er }}$ janvier 2017, les communes peuvent exonérer les propriétés non bâties sur lesquelles les propriétaires ont grevé une obligation réelle environnementale de la taxe foncière. Il n'en demeure pas moins, qu'en dépit des nombreuses propositions doctrinales ${ }^{46}$ qui avaient déjà été faites dans le sens d'une consécration de l'obligation réelle, c'est bien la Cour de cassation qui la première ouvrait la porte d'une potentialité nouvelle visant la protection d'un usage écologique de l'immeuble via la création d'une droit réel de jouissance spéciale ${ }^{47}$. Enfin, il est besoin de tempérer l'efficacité de cette nouvelle obligation réelle puisqu'elle nécessitera le plus souvent l'accord du preneur à bail rural et que, pour l'heure, il semble difficile d'expliquer aux intéressés l'opportunité directe du

43. Cass. civ. $3^{\mathrm{e}}, 31$ octobre 2012, n 11-16.304, RTD civ., 2013, p. 141, obs. Dross W., RDC 2013, p. 584, note Libchaber R. ; Cass. civ. $3^{\mathrm{e}}, 28$ janvier 2015, $\mathrm{n}^{\circ} 14-10.013$, D. 2015, p. 599, note Mallet-Bricout B., RTD civ. 2015, p. 413, note Dross W., Defrénois 2015, p. 419, note Andreu L., Thomassin N., Cass. civ. $3^{\mathrm{e}}$, 8 sept. 2016, ${ }^{\circ}$ 14-26.953, RDI, 2016, p. 598, obs. Bergel J.-L., RTD civ. 2016, p. 894, obs. Dross W., voy. également d'Avout L., Mallet-Bricout B., « De l'autonomie, de la durée et des causes d'extinction des droits réels de jouissance spéciale », D. 2017, p. 134 (le droit réel de jouissance spéciale peut être calqué sur la durée de vie d'une personne morale) ; la doctrine rapproche parfois la constitution d'un droit réel de jouissance spéciale et la technique de fiducie-gestion, voy. par ex. Mekki M., «Loi biodiversité et droit de la construction», RDI 2016, p. 576.

44. V. Reboul-Maupin N., Grimonprez B., « Les obligations réelles environnementales : chronique d'une naissance annoncée », D. 2016, p. 2074.

45. Art. 662 et ss. CGI.

46. Martin G.-J., « Pour l'introduction en droit français d'une servitude conventionnelle ou d'une obligation propter rem de protection de l'environnement ", RJE 2008, ${ }^{\circ}$ spécial, p. 123 ; Avant-projet de réforme du droit des biens, Périnet-Marquet (dir.), $\mathrm{v}^{\circ}$ art. $608:$ « Le propriétaire peut consentir, sous réserve des règles d'ordre public, un ou plusieurs droits réels conférant le bénéfice d'une jouissance spéciale d'un ou de plusieurs de ses biens »; Raoult P., Barthod C., Graffin V., " Trame verte et bleue », rapport remis au ministre de l'Écologie dans le cadre du Comité opérationnel n 11 du Grenelle de l'environnement, le 14 mars 2008 , spéc. p. 21 et s..

47. En ce sens v. Mekki M., «Les virtualités environnementales du droit réel de jouissance spéciale », $R D C \mathrm{n}^{\circ} 1$, p. 105. 
dispositif ${ }^{48}$. Dans un même sens, la mise en œuvre de l'obligation sera limitée par la préservation des " droits liés à l'exercice de la chasse » et « ceux relatifs aux réserves cynégétiques». La pratique de la chasse s'oppose donc à la constitution de mesures de compensation par le biais de la constitution d'une obligation réelle.

La compensation comme l'obligation réelle environnementale sont porteuses de grandes promesses, de nombreuses potentialités et de limites, certaines mises en exergue par le contrôle de la compensation.

\section{B. LE CONTRÔLE DE LA COMPENSATION}

Pour que l'obligation de compensation ne reste pas un vœu pieux il sera besoin d'établir un véritable suivi. Ce qui implique, pèle mêle, de contrôler l'utilisation de la stratégie ERC mise en œuvre par les maîtres d'ouvrage, d'associer les collectivités territoriales à l'identification des espaces à fort potentiels de gain écologique, localiser en priorité les mesures compensatoires sur des territoires cohérents avec la trame verte et bleue ${ }^{49}$, de suivre localement la mise en œuvre des mesures de compensation ${ }^{50}$, de proposer une grille de lecture harmonisée des méthodes de suivi etc.

Par ailleurs, il paraît naïf de croire que la compensation sera effective si son inexécution totale ou partielle n'est pas accompagnée de sanctions. À ce titre les articles L. 163-4 et L. 171-8 du Code de l'environnement prévoient une panacée que l'on retrouve de manière assez classique dans d'autres pans du droit de l'environnement ${ }^{51}$ :

48. Comp. Van Lang A., « La loi biodiversité du 8 août 2016 : une ambivalence assumée », AJDA 2016, p. 2381 : « La participation du preneur à la constitution de l'obligation en question peut lui permettre de soumettre son accord à une baisse de son loyer ou autres avantages » (mais dans ce cas c'est le propriétaire qui pourrait ne pas s'y retrouver)

49. L'institution de la trame verte et bleue vise à " enrayer la perte. de biodiversité en participant à la préservation, à la gestion et à la remise en bon état des milieux nécessaires aux continuités écologiques, tout en prenant en compte les activités humaines, notamment agricoles, en milieu rural » (art. L. 371-1-I C. env.). Les « trames » représentent des grands ensembles naturels permettant d'avoir une meilleure visibilité de la biodiversité à protéger sur un territoire donné.

50. Cf. Rapport du Sénat n 517 (2016-2017) de R. Dantec, Commission d'enquête sur les mesures de compensation des atteintes à la biodiversité engagée sur des grands projets d'infrastructures. Le rapport suggère à ce propos la création d'agences régionales pour la biodiversité.

51. Martin G.-J., « Les unités de compensation dans la loi n 2016-1087 du 8 août 2016 pour la reconquête de la biodiversité, de la nature et des paysages », art. préc. : «(...) rien 
la première étape est celle de la mise en demeure, dans un délai déterminé par l'autorité administrative compétente (le préfet, par compétence liée) ; le deuxième étape, conditionnée au non déferrement du débiteur de l'obligation de compensation à la mise en demeure, est celle de l'exécution de la mesure qui se fera soit en faisant appel à un opérateur (rien n'est dit sur la carence du maître d'ouvrage qui ne pourrait pas se tourner vers un opérateur), soit par l'achat d'unité de compensation. Dans tous les cas l'autorité administrative « peut » soumettre une autorisation à la constitution de « garanties financières » destinées à assurer, d'une manière ou d'une autre, la compensation. Un manquement à l'une de ces obligations peut également donner lieu à une procédure de consignation, à une amende administrative et/ou à des sanctions pénales.

En outre, même si la compensation était parfaitement réalisée, la loi ne règle pas le problème de son suivi. L'achat d'unités, par exemple, suggère une difficulté d'évaluation concernant les effets globaux de la mesure et certainement une perte de cohérence écologique. La limitation dans le temps des contrats de compensation n'est pas toujours compatible avec la pérennisation des mesures compensatoires. Si l'opérateur de compensation doit faire face à une mutation de propriété contredisant l'affectation écologique d'un site, est-ce que l'administration pourra ordonner au maître d'ouvrage de recommencer la compensation?

Le contrôle de ces mesures de compensation soulève donc de nombreux problèmes que l'apport des services écosystémiques à la compensation pourrait accentuer.

ne ressemble à une vraie régulation dans les texte actuels. Plus exactement, la régulation mise en œuvre frappe par son classicisme (...) et son inadaptation aux vrais enjeux qu'elle omet de prendre en compte. Les opérateurs de compensation, en tant que tels, ne font l'objet d'aucun agrément. L'agrément des sites naturels de compensation est, pour sa part, loin de répondre aux besoins. On peut, parfois, avoir le sentiment que les auteurs des textes ont raisonné à propos de ces sites, comme ils raisonnent à propos des installations classées pour la protection de l'environnement, en omettant de prendre en compte la dimension de droit économique, qu'imposerait pourtant l'émergence d'un nouveau secteur marchand ». 


\section{LA PERTINENCE DE LA COMPENSATION DES SERVICES ÉCOSYSTÉMIQUES}

La compensation, dans son objet, n'englobe pas directement les services écosystémiques. Elle est réduite aux fonctions (A) par la lettre de la loi. Il n'en demeure pas moins un lien entre les deux que nous tâcherons de dé-couvrir. Nous envisagerons ensuite les perspectives critiques de ce lien (B) entretenant une certaine ambiguïté entre les notions de fonction et de service.

\section{A. LA RÉDUCTION DE LA COMPENSATION AUX FONCTIONS ÉCOLOGIQUES}

Le droit de la compensation ex ante ne se préoccupe que des atteintes portées à l'environnement. En effet, les premières moutures du projet de loi concernant la réécriture de l'article L. 110-1 $2^{\circ}$ du Code de l'environnement faisait référence aux « services et fonctions écosystémiques affectés $\|^{52}$, avant que la compensation ne soit cantonnée aux « atteintes qui n'ont pu être évitées ni réduites, en tenant compte des espèces, des habitats naturels et des fonctions écologiques affectées ». Cette suppression est perçue par Mélodie Fèvre comme « une sorte de malaise face à la manipulation $\|^{53} \mathrm{de}$ la notion de services écosystémiques qui renvoie à une confusion entre fonction et service. Or, il s'agit là de deux visions bien distinctes : dans le cadre des fonctions c'est encore la biodiversité que l'on souhaite protéger, dans le cadre des services c'est une utilité particulière et surtout bénéficiant à une activité humaine, donc nécessairement subjective. Dès lors, on comprend pourquoi il serait difficile de compenser directement l'atteinte aux services écosystémiques : le coût du service dépend du bénéfice qu'il produit, le faisant ainsi varier en fonction de l'utilité qu'en retirent

52. Assemblée Nationale, Projet de loi relatif à la biodiversité (première lecture), Texte de la Commission du développement durable et de l'aménagement du territoire, $\mathrm{n}^{\circ} 2064$, Annexe au rapport, 26 juin 2014, p. 4

53. V. supra Fèvre M., "Les « services écosystémiques », une notion fonctionnelle »; adde Fèvre M., 2016, Les services écologiques et le droit, Une approche juridique des systèmes complexes, Doussan I. (dir.), Université Côté d'Azur, p. 411 et ss. : « (...) les fonctionnalités écologiques et les services écologiques ne correspondraient-ils pas à des enjeux de la biodiversité, qui nécessitent par ailleurs des indicateurs appropriés ? Certaines écologues soulignent en effet que les deux termes n'induisent pas les mêmes incidences, et par conséquent les mêmes exigences s'agissant de la mise en œuvre des mesures compensatoires (...). C'est ce qui expliquerait que les services soient retirés du texte régissant les atteintes soumises à compensation, parce que certaines incidences $d u$ concept constituent aujourd'hui des limites à sa mise en œuvre ». 
les bénéficiaires. Ceci étant observé, il n'en reste pas moins que, concernant la compensation ex post cette fois-ci, le dommage écologique intègre dans sa définition la notion de "service écologique », ce qui complique un peu plus la clarté de la distinction. L'article L. 161-1 $4^{\circ}$ du Code de l'environnement dispose bien que " constituent des dommages causés à l'environnement au sens du présent titre les détériorations directes ou indirectes mesurables de l'environnement qui affectent les services écologiques, c'est-à-dire les fonctions assurées par les sols (...)».

De la même façon, l'obligation réelle environnementale n'a que pour finalité « le maintien, la conservation, la gestion ou la restauration d'éléments de la biodiversité ou de fonctions écologiques ». Les services écosystémiques ne sont pas évoqués. Pourtant, nous pensons que la consécration d'autres mécanismes permettrait une prise en compte indirecte des services par la compensation. Tel pourrait être le cas si l'on considérait le principe de solidarité.

Désormais érigé au rang de principe, la solidarité ${ }^{54}$ « (...) appelle à prendre en compte, dans toute prise de décision publique ayant une incidence notable sur l'environnement des territoires concernés, les interactions des écosystèmes, des êtres vivants et des milieux naturels ou aménagés » (art. L. 110-1-II $6^{\circ}$ C. env.). En appliquant ce principe à l'aire de compensation il est tout-à-fait permis de penser que la personne qui mettra en œuvre la mesure devra tenir compte de l'interdépendance des fonctions écologiques entre plusieurs territoires. Plus encore, le débiteur de compensation devra tenir compte des " interactions des écosystèmes ", des " êtres vivants » et des «milieux naturels ou aménagés ». À titre d'exemple, il serait permis de penser qu'une collectivité territoriale ayant une biodiversité riche et devant supporter le coût d'une telle mesure, pourra demander à une collectivité voisine qui bénéficie des services écosystémiques générés par cette biodiversité de participer à sa conservation $^{55}$. Plus généralement, le fonds mitoyen qui bénéficierait

54. À l'instar du principe de compensation naguère, le principe de solidarité écologique était à l'origine un outil permettant de répondre à la question juridique particulière des critères de zonage de l'aire optimale d'adhésion des Parcs nationaux (art. L. 331-1 al. 2 C. env.). Dans ce dernier cadre, l'approche en termes de proximité géographique s'avérait insuffisante.

55. Voir Icher L., « La dépense publique en matière environnementale : l'exemple de la protection des sols et des services ", infra, à propos de la loi montagne du 28 décembre 2016. 
d'une mesure de compensation au travers une amélioration d'un service écosystémique pourrait se voir demander une participation financière par l'aménageur. Depuis son introduction pour les Parcs nationaux le « principe » de solidarité n'a fait l'objet d'aucun développement jurisprudentiel à notre connaissance. Il faudra donc attendre de voir comment le juge se saisira de ce nouvel outil. Si le contenu normatif de ce nouveau principe est encore très incertain, il n'offre pas moins une vision dynamique de la biodiversité permettant la considération des services écosystémiques dans la mise en œuvre des mesures de compensations.

Parmi les principes généraux du droit de l'environnement, le principe de complémentarité ${ }^{56}$ pourrait également rapprocher compensation et services écosystémiques. L'article L. 110-1 II $8^{\circ}$ du Code de l'environnement constate que les acteurs économiques ${ }^{57}$ prennent en compte les « interactions écosystémiques garantissant, d'une part, la préservation des continuités écologiques et, d'autre part, des services environnementaux qui utilisent les fonctions écologiques d'un écosystème pour restaurer, maintenir ou créer de la biodiversité $\gg$. En laissant de côté l'absence de normativité de la disposition ${ }^{58}$, on peut relever les conséquences bénéfiques qu'apportent certaines activités agricoles. En raisonnant de manière prospective, c'est la place de l'agriculteur-producteur de biodiversité ${ }^{9}$ qu'il convient de questionner. Est-ce que les pratiques se rapprochant d'une agriculture de conservation ou, à tout le moins, d'une agriculture biologique, ne peuvent-elles pas être considérées comme un voie possible de compensation? Si, à l'avenir, une réponse positive était apportée cela permettrait de reconsidérer pleinement le rôle de l'agriculteur dans

\footnotetext{
56. Pour la critique de la consécration de la complémentarité au titre de principe fondamental voy. Doussan I., "Vive la complémentarité de l'agriculture et de l'environnement », in Cans Ch., Cizel O., Loi Biodiversité, ce qui change en pratique, op. cit., p. 77.

57. Plus particulièrement les agriculteurs semble-t-il, même si le principe intègre l'article L110 ; voy. Van Lang A., " La loi Biodiversité du 8 août 2016 : une ambivalence assumée », précité.

58. Champeil-Desplats V., « N'est pas normatif qui peut. L'exigence de normativité dans la jurisprudence du Conseil Constitutionnel », Cahiers du Conseil Constitutionnel, $\mathrm{n}^{\circ} 21$, janv. 2007 ; du même auteur, Méthodologies du droit et des sciences du droit, Dalloz, 2014, p. 262, n 422 et s. spéc. $n^{\circ} 431$; Comp. avec la décision n 2016-737 DC du 4 août 2016 du Conseil constitutionnel, relative au contrôle a priori de la loi pour la reconquête de la biodiversité, de la nature et des paysages, voy. spéc. le considérant $n^{\circ} 7$ relatif à la normativité du principe de non-régression.

59. Labous K., Gruger H., « Produire de la biodiversité : un avenir pour les agriculteurs », Dr. Env. n 259, sept. 2017, p. 291.
} 
la mise en œuvre des mesures compensatoires et dans la protection des sols qu'il exploite. Partant il favoriserait également la protection des services qu'il en retire. Une des conséquences profitables serait un allégement de la pression foncière dans la mise en œuvre des mesures de compensation, déjà évoquée.

\section{B. Perspectives critiques de la compensation éCo-Centrée}

Concernant la réception du nouveau dispositif, la loi Biodiversité a connu une longue gestation mais donne naissance à 174 articles et consacre de nombreux mécanismes qui pourront s'influencer et dont il est, pour l'heure, impossible d'en étudier la portée. Le temps de l'exégèse cédera progressivement sa place à celui du contentieux et c'est à ce moment que de nombreuses réponses seront apportées.

D'un point de vue technique, le vocabulaire utilisé par la loi nouvelle révèle une approche essentiellement comptable du droit de l'environnement puisque les mesures de compensation doivent avoir pour objectif, à tout le moins, une " absence de perte nette », voire un " gain de biodiversité » (art. L. 110-1-II $2^{\circ}$ C. env.). L'idée, concernant les sites naturels de compensation, est donc de remplacer une unité de biodiversité par une autre. Mais cela implique, en droit, une fongibilité ${ }^{0}$ bien difficile à concevoir en pratique. « Le sol, fait remarquer Mme Desrousseaux, est un bien cessible, appropriable et disponible, mais sa qualité, c'est-à-dire les services écologiques qui y sont liés, ne peut en être détachée de manière à rendre possible leur destruction en un lieu et leur compensation en un autre, sans perdre leur substance $\rangle^{61}$. En dépassant cet aspect lié à la notion de fongibilité, on pourra même voir dans la compensation opérée sur un site différent une contradiction avec le principe de solidarité écologique visant la prise en compte de l'interdépendance entre les écosystèmes qui revêt nécessairement une dimension territoriale. Le législateur prenant acte de cette incohérence demande à ce que la compensation s'opère « en priorité » sur le " site endommagé » ou, « en tout état de cause », "à proximité ». Ce caractère de la compensation a conduit la doctrine à une critique parfois très appuyée

60. Zenati Fr., Revet Th., Les biens, PUF, $3^{e}$ éd., $§ 152$ : « La fongibilité est la comparaison de laquelle il résulte que deux biens sont de mêmes espèce et qualité, ce qui rend possible le remplacement de l'un par l'autre ».

61. Desrousseaux M., 2016, La protection juridique de la qualité des sols, LGDJ, t. 13, p. 309. 
à l'instar de Mme Camproux-Duffrène pour qui « compenser par anticipation, c'est nier le fonctionnement des écosystèmes et la spécificité de chaque écosystème. Compenser n'a pas comme effet de revenir à l'équilibre antérieur puisqu'il existe encore, c'est organiser une destruction future et certaine et en prévoir des contreparties $»^{62}$. L'acquisition d'« unités » renforce cette idée et semble représenter une dénégation complète de la spécificité bio-physico-chimique de chaque sol $^{63}$ qui fera l'objet d'une mesure de compensation. Toute la question réside dans la pertinence et l'efficacité de ces « contreparties ${ }^{64}$.

En définitive, les difficultés principales seraient de trois ordres. Il y a d'abord deux logiques qui s'opposent, elles tiennent à l'ontologie du droit de l'environnement : protège-t-on le sol per se ou protège-t-on le service rendu ? Plus précisément, en attribuant une valeur d'usage à une fonction écologique le législateur n'a-t-il pas introduit un biais entre fonction et service ? On aurait en effet du mal à concevoir la fongibilité d'une fonction convertissable en unité de biodiversité sans penser que les services écosystémiques ne le seraient pas ${ }^{65}$. La loi n'est pas claire à ce sujet : elle intègre dans le patrimoine naturel la notion de service mais l'exclut quand il s'agit de compensation. Pourtant, il semble difficile de disjoindre les deux au moment où l'on réalise les mesures.

Il y a, ensuite, dans la loi et dans ses décrets d'application, un manque de précisions concernant la mise en œuvre et le contrôle

62. Camproux-Duffrène M.-P., « Le marché d'unités de biodiversité : questions de principe », RJE 2008, p. 87 ; adde Van Lang A., « La compensation des atteintes à la biodiversité : de l'utilité technique d'un dispositif éthiquement contestable », RDI 2016, p. 586.

63. Voy. Berthet E., Concevoir l'écosystème, un nouveau défi pour l'agriculture, Paris, Presses des MINES, 2014.

64. V. Maris V., Nature à vendre, Les limites des services écosystémiques, Quae, 2014, spéc. p. 58 et ss. : l'auteur y dénonce une « marchandisation de la nature » (« la transformation d'un bien ou d'un service en marchandise implique trois prérequis : que l'objet de l'échange soit réductible, appropriable et substituable. Nous allons voir maintenant que, en ce qui concerne les services écosystémiques, chacun de ces prérequis pose problème »); du même auteur, Philosophie de la biodiversité, Petite éthique pour une nature en péril, Buchet/ Castel, 2016.

65. V. Martin G.-J., « Les « biens-environnements ». Une approche par les catégories juridiques », RIDE 2/2015, t. XXIX, p. 139 : « (...) le bien et les services (par exemple la biodiversité et les services qu'elle fournit) demeurent alors dans leur matérialité hors du commerce juridique, mais dans des titres dématérialisés représentent l'investissement nécessaire à leur production et à leur gestion. Ces titres sont évidemment des biens dans le commerce juridique $»$. 
des mesures compensatoires. Cela tient à la problématique même du sol et des services y étant attachés : l'ancrage à la spécificité d'une terre particulière ne se laisse que très difficilement enfermé dans le cadre général de la loi. La complexité et la spécificité de chaque sol appellerait presque un régime de compensation particulier que le caractère général et impersonnelle de la loi a pour l'instant du mal à protéger. Il y a en plus un manque en terme d'indicateurs qui ne permet de chiffrer et de qualifier les gains de biodiversités corrélatives aux pertes ${ }^{66}$. Le législateur aurait livré le dispositif sans la notice d'utilisation. Pour une grille de lecture commune il faudra très certainement se tourner vers les outils de budgétisation des politiques publiques ${ }^{67}$.

Enfin, la réflexion sur la compensation s'insère dans un champ notionnel nouveau qui n'a, pour l'heure, pas encore subi la patine du temps. Équivalence, service, fonction, additionnalité, solidarité, proximité, gain, perte nette etc. sont autant d'outils avec lesquels le juriste devra construire une protection plus efficace des sols tout en assurant la pérennité des services qu'ils rendent à celles et ceux qui l'exploitent.

66. V. Regnery B., 2013, Les mesures compensatoires pour la biodiversité. Conception et perspectives d'application, thèse de doctorat en Sciences de l'Ecologie, Université Pierre et Marie Curie.

67. V. infra Icher L., « La dépense publique en matière environnementale : 1'exemple de la protection des sols et des services ». 



\title{
La commande publique de compensation environne- mentale : un impensé de la loi Biodiversité
}

\author{
Hélène HOEPFFNER \\ Professeur, Université Toulouse Capitole \\ IEJUC, F-31000
}

Le Code de l'environnement prévoit, dans certaines hypothèses, qu'un porteur de projet de construction et de travaux d'infrastructures publiques ou de bâtiments privés soit contraint de respecter une séquence dite ERC : éviter, réduire, compenser ${ }^{1}$. Celle-ci lui impose, lors de la mise en œuvre d'un projet artificialisant des terres, de minimiser ses impacts environnementaux. Il est tenu d' « éviter les effets négatifs notables du projet sur l'environnement ou la santé humaine et de réduire les effets n'ayant pu être évités ». Puis, si ces effets négatifs « n'ont pu être ni évités ni suffisamment réduits ", il doit mettre en œuvre des mesures de compensation c'est-à-dire mener « un ensemble d'actions écologiques permettant de contrebalancer les pertes de biodiversité dues à des projets d'aménagement, lorsque l'aménageur n'a pu ni éviter ces pertes ni les réduire $»^{2}$.

L'obligation de compenser n'est pas nouvelle ${ }^{3}$. Elle figure dans notre droit depuis la loi $\mathrm{n}^{\circ} 76-629$ du 10 juillet 1976 relative à la protection de la nature. Elle a ensuite été progressivement étendue, notamment par les textes réformant l'étude d'impact ${ }^{4}$. Le résultat de

1. Pour le champ d'application de cette séquence, v. Lucas M., Etude juridique de la compensation économique, thèse Strasbourg, 2012, LGDJ Biblio. Droit Urba. Envir., 2015. 2. Compenser les atteintes à la biodiversité : expériences internationales et enseignements pour la France, 2012 (en ligne).

3. Sur l'historique de l'évolution de la compensation écologique, v. Lucas M., thèse préc., pp. 11 et ss. et pp. 103 et ss.

4. Loi $\mathrm{n}^{\circ}$ 2010-788 du 12 juill. 2010 portant engagement national pour l'environnement ; décret $\mathrm{n}^{\circ}$ 2011-2019 du 29 déc. 2011 portant réforme des études d'impact ; ordonnance $n^{\circ} 2016-1058$ du 3 août 2016 et décret $n^{\circ} 2016-1110$ du 11 août 2016 relatifs à la 
cet essor est cependant apparu en demi-teinte. On pouvait s'inquiéter de l'éparpillement des dispositifs formalisant cette obligation. D'aucuns n'ont d'ailleurs pas manqué de souligner à quel point la mise en œuvre des mesures compensatoires était laborieuse ${ }^{5}$. Cela a conduit le Gouvernement à publier une doctrine nationale, sous la forme de guides méthodologiques à caractère non contraignant ${ }^{6}$, assortie de lignes directrices ${ }^{7}$ et à mettre en place des groupes de travail ministériels ${ }^{8}$. Cela a aussi conduit à plusieurs réformes et, en particulier, à la loi $n^{\circ} 2016-1087$ du 8 août 2016 relative à la reconquête de la biodiversité, de la nature et des paysages ${ }^{9}$.

Ce texte donne force de loi à la séquence ERC dans un nouveau chapitre du Code de l'environnement (chap. III du titre VI du livre Ir) réglementant la compensation ex ante, c'est-à-dire l'ensemble des actions mises en œuvre pour contrebalancer les dommages induits - mais non encore réalisés - par un projet d'aménagement, plan ou programme sur l'environnement qui n'ont pas pu être suffisamment évités ou réduits. En tête de ces nouvelles dispositions, l'article L. 163-1 du Code de l'environnement définit l'obligation de compensation $^{10}$. Il en fait une déclinaison du principe de prévention des atteintes à la biodiversité. In fine, il précise que « si les atteintes liées au projet ne peuvent être ni évitées, ni réduites, ni compensées de façon satisfaisante, celui-ci n'est pas autorisé en l'état ».

modification des règles applicables à l'évaluation environnementale des projets, plans et programmes.

5. Lucas M., « La compensation environnementale, un mécanisme inefficace à améliorer », RJE 2009, n 1 , p. 59.

6. MEDDTL, Doctrine relative à la séquence éviter, réduire et compenser les impacts sur le milieu naturel, mai 2012 ; CGDD, Lignes directrices nationales sur la séquence éviter, réduire et compenser les impacts sur les milieux naturels, MEDDE, oct. 2013.

7. CGDD, Lignes directrices nationales sur la séquence éviter, réduire et compenser les impacts sur les milieux naturels, op. cit..

8. Rapport du Groupe de travail "Améliorer la séquence Eviter - réduire - compenser ", janv. 2015 ; Moderniser l'évaluation environnementale, mars 2015.

9. V. not. Grimonprez B., « La compensation écologique d'après la loi biodiversité », Dr. Patr., $1^{\text {er }}$ nov. $2016, \mathrm{n}^{\circ} 263$; Martin, G.-J., « La compensation écologique : de la clandestinité honteuse à l'affichage mal assuré », RJE, 2016, n 4, p. 606 ; Van Lang, A. «La compensation des atteintes à la biodiversité : de l'utilité technique d'un dispositif éthiquement contestable », RDI 2016, p. 586 ; dossier de la revue $E E I, 2017, \mathrm{n}^{\circ} 6$; Dupont V., Lucas M., « La loi pour la reconquête de la biodiversité : vers un renforcement du régime juridique de la compensation écologique ?», Cahiers Droit Sciences \& Technologies, 2017, $\mathrm{n}^{\circ} 7, \mathrm{p} .143$.

10. Combe M., « Le régime juridique de l'obligation de compensation écologique », EEI $2017, n^{\circ} 6$, dossier 8 . 
Le même article détaille ensuite les instruments juridiques permettant de mettre en œuvre cette obligation. Le maître d'ouvrage dispose d'un choix entre trois méthodes pouvant être mises en œuvre de manière alternative ou cumulative. Deux d'entre elles reposent sur la technique contractuelle ${ }^{11}$. Le maître d'ouvrage peut effectuer luimême les mesures de compensation, confier, par contrat, l'exécution des mesures de compensation à un " opérateur de compensation" ou se tourner vers un professionnel de la compensation « qui aura, sur ses propres sites, produit de la valeur écologique traduite en titres (les unités de compensation) et les lui acheter pour remplir son obligation $»^{12}$.

Il n'y a pas lieu ici de reprendre les analyses de ces contrats réalisées en droit de l'environnement, par le prisme du droit civil ${ }^{13}$. Il s'agit de les confronter au droit des contrats administratifs et, plus précisément, au droit de la commande publique.

Le législateur est resté muet sur la nature juridique de ces contrats, contrairement à ce qu'il a fait il y a quelques années pour les contrats Natura 2000 en soumettant les litiges y afférant à la juridiction administrative (art. L. 414-4-I al 3 C. envir.). A. Van Lang ${ }^{14}$ regrette ce silence, estimant que « compte tenu de la finalité de compensation de la biodiversité (assurément d'intérêt général), il eût été judicieux que la loi identifie un contrat administratif, quelle que soit la nature des parties ».

Le présent article entend proposer un autre raisonnement en se posant la question de savoir dans quelle mesure ces contrats de compensation peuvent être qualifiés de contrats de la commande publique ${ }^{15}$, c'est-à-dire un contrat par lequel « une personne

11. Lucas M., « Le contrat au service de la compensation écologique », EEI, 2017, $\mathrm{n}^{\circ}$ 6, dossier 11 ; Hautereau-Boutonnet M., " La reconquête de la biodiversité par la conquête du droit civil... », JCP G 2016, 948 ; Monteillet V., La contractualisation du droit de l'environnement, thèse Montpellier, NBT Dalloz 2017, $\mathrm{n}^{\circ} 48$ et ss.

12. Lucas, M., précité.

13. Cf. supra Alidor B. «Compensation et services écosystémiques ».

14. Van Lang A., art. préc. V. également : Monteillet S., thèse préc. p. 88.

15. L'ordonnance $n^{\circ} 2009-515$ du 7 mai 2009 définit les contrats de la commande publique comme ceux par lesquels une autorité adjudicatrice passe un contrat destiné à satisfaire ses besoins ou ceux des usagers « ayant pour objet l'exécution de travaux, la livraison de fournitures ou la prestation de services, avec une contrepartie économique constituée par un prix ou un droit d'exploitation ou la délégation d'un service public » et dont la passation est soumise à des règles de publicité et de mise en concurrence. 
publique cherche à se procurer un bien ou un service, y compris ce service d'une nature un peu particulière qui consiste à gérer avec une relative autonomie une activité ou un équipement $\rangle^{16}$. Il est traditionnellement admis que les contrats de la commande publique se répartissent en deux grandes catégories. D'une part, les marchés publics qui sont « les contrats à titre onéreux, conclus par écrit entre un ou plusieurs opérateurs économiques et un ou plusieurs pouvoirs adjudicateurs (appelés acheteurs), pour répondre à leurs besoins en matière de travaux, de fournitures ou de services » (art. 4 ordonnance 23 juillet 2015). D'autre part, les contrats de concessions qui « sont les contrats conclus par écrit, par lesquels une ou plusieurs autorités concédantes ", c'est-à-dire un ou plusieurs pouvoirs adjudicateurs ou autorités adjudicatrices (art. 8, ordonnance 29 janv. 2016) « confient l'exécution de travaux ou la gestion d'un service à un ou plusieurs opérateurs économiques, à qui est transféré un risque lié à l'exploitation de l'ouvrage ou du service, en contrepartie soit du droit d'exploiter l'ouvrage ou le service qui fait l'objet du contrat, soit de ce droit assorti d'un prix » (art. 5, ibid).

Si tel est le cas, lorsqu'ils sont passés par des personnes morales de droit public, ils sont nécessairement des contrats administratifs dans la mesure où les articles 3 des ordonnances du 23 juillet 2015 relative aux marchés publics et du 29 janvier 2016 relative aux contrats de concessions qualifient les marchés publics et les contrats de concession passés par les personnes morales de droit public de contrats administratifs. Comme en droit de la commande publique, il existerait ainsi une dichotomie entre, d'une part, les contrats conclus par un maître d'ouvrage public, qui seraient administratifs par détermination de la loi et, d'autre part, ceux conclus par un maître d'ouvrage privé, qui seraient des contrats de droit privé ${ }^{17}$.

A priori, il ne fait guère de doute que les contrats de compensation, qu'il s'agisse des contrats conclus avec un opérateur de compensation en vue que celui-ci réalise des prestations de compensation ou des contrats ayant pour objet l'acquisition d'unités de compensation tendent à satisfaire le besoin du maître de l'ouvrage débiteur de

16. Delacour E., «Les fondements et les orientations de la commande publique : du marché public à l'achat public », CMP 2005, étude 6 ; Eckert G., « Réflexions sur l'évolution des contrats publics », RFDA 2006, p. 238.

17. Ce qui signifie que leurs régimes juridiques sont distincts et que leurs contentieux relèvent de deux juridictions différentes. 
l'obligation de compensation. Il convient néanmoins de s'en assurer (I). Il convient ensuite de confronter leur régime juridique, issu du Code de l'environnement, aux règles de la commande publique (II).

\section{L'IDENTIFICATION DE LA COMMANDE PUBLIQUE DE COMPENSATION}

Pour que le contrat de compensation puisse être qualifié de contrat de la commande publique, il faut d'une part, que le maître d'ouvrage, débiteur de l'obligation de compensation, soit susceptible d'être qualifié de pouvoir adjudicateur (A) et d'autre part, que le contrat ait pour objet de satisfaire le besoin de celui-ci en matière de travaux, de fournitures ou de services (B).

\section{A. LE CRITÈRE ORGANIQUE : LE DÉBITEUR DE L'OBLIGATION DE COMPENSATION, UN POUVOIR ADJUDICATEUR}

L'obligation de compenser s'impose au « maître d'ouvrage », c'està-dire à « l'auteur d'une demande d'autorisation concernant un projet privé ou l'autorité publique qui prend l'initiative d'un projet » (art. L. 122-1-I- $1^{\circ} \mathrm{C}$. envir.). A défaut de pouvoir exécuter lui-même cette obligation, il contracte avec un " opérateur de compensation », c'est-à-dire une personne « chargée de mettre en œuvre les mesures de compensation des atteintes à la biodiversité et de les coordonner à long terme » (art. L. 163-1-III), sans néanmoins préciser le statut de celui-ci (public ou privé) ou ses compétences. Cet opérateur de compensation peut ainsi aussi bien être un agriculteur, qu'une association, un bureau d'études, un conservatoire d'espaces naturels ou une collectivité territoriale ${ }^{18}$. Il désigne une entité dotée d'un savoir-faire de terrain pour recréer des milieux, déplacer et réimplanter des espèces.

Aux termes des ordonnances des 23 juillet 2015 et 29 janvier 2016 relatives respectivement aux marchés publics et aux contrats de concession, les règles de la commande publique s'imposent aux « pouvoirs adjudicateurs » lorsqu'ils contractent avec un

18. Lucas M., « Collectivités locales et compensation écologique, quelles perspectives ?», Droit et Ville, 2017, à paraître. 
« opérateur économique ». En droit français, la notion de pouvoir adjudicateur désigne trois catégories d'entités (art. 10, ordonnance 23 juill. 2015) : les « personnes morales de droit public », quels que soient leur forme juridique et leur objet (ex. les collectivités territoriales, les établissements publics) ; les « personnes privées relevant de la catégorie des organismes de droit public » (ex. des sociétés d'économie mixte); et «les organismes de droit privé constitués par des pouvoirs adjudicateurs en vue de réaliser certaines activités en commun ", c'est-à-dire des entités ad hoc, créées en vue d'une coopération publique. La notion d'opérateur économique désigne quant à elle « toute personne physique ou morale, publique ou privée, ou tout groupement de personnes doté ou non de la personnalité morale, qui offre sur le marché la réalisation de travaux ou d'ouvrages, la fourniture de produits ou la prestation de services $»$ (art. 13, ordonnance 23 juill. 2015). Il est une entité qui offre une prestation « sur un marché », la jurisprudence française ayant précisé qu'il doit intervenir « sur un marché concurrentiel $»^{19}$.

Il ne fait guère de doute que le contrat de compensation, conclu entre un maître d'ouvrage et un opérateur de compensation peut remplir ce critère organique du contrat de la commande publique. Le maître d'ouvrage au sens du droit de l'environnement peut être un pouvoir adjudicateur (ex. une collectivité territoriale). L'opérateur de compensation peut être un opérateur économique au sens du droit de la commande publique car il devrait intervenir sur un marché concurrentiel : celui de la réalisation de prestations de compensation. Tel est le cas de CDC Biodiversité, filiale de la CDC ou du groupement d'intérêt public créé par le Département des Yvelines spécialisé dans la compensation ${ }^{20}$.

Un doute peut en revanche être émis concernant le gestionnaire d'un site naturel de compensation. Il n'est guère contesté que, selon le sens commun, il y a bien un marché des unités de compensation dès lors qu'il y a une offre et une demande de ce bien particulier qu'est

19. CE, sect., 6 avr. 2007, Cne Aix-en-Provence, $\mathrm{n}^{\circ} 284736$ : v. not. CMP 2007, comm. 191, Eckert G. ; D. 2007. 2617, Clamour G. ; RFDA 2007, p. 812, concl. Séners F., et 821, note Douence, J.-C. ; CE 3 fév. 2012, Cne Veyrier du Lac, n 353737 ; AJDA 2012, p. 555, Richer L. ; BJCP 2012, p. 153, concl. Dacosta B. ; CMP 2012, comm. 78, Zimmer W. ; Dr. Adm. 2012, comm. 33, Brenet F. ; RTD eur. 2012. 940, Muller E.

20. http://lagazette-yvelines.fr/2017/02/16/biodiversite-collectivites-sassocient-compenserpertes/ 
l'unité de compensation. Mais, il n'y a pas de marché au sens des marchés qui existent en matière financière, de matières premières ou de quotas d'émission de gaz à effet de serre ${ }^{21}$, notamment parce que l'émission de chaque unité n'est pas interchangeable. Elle est attachée aux caractéristiques environnementales de chaque site et le marché sur lequel elle s'achète est nécessairement local. Cette exigence figure expressément à l'article L. 163-1 du Code de l'environnement qui dispose que « les mesures de compensation sont mises en œuvre en priorité sur le site endommagé, ou, en tout état de cause, à proximité de celui-ci, afin de garantir ses fonctionnalités de manière pérenne ». Il nous semble qu'au regard du droit de la commande publique néanmoins, le gestionnaire d'un tel site émettant des unités de compensation exerce bien une activité économique au sens de la jurisprudence européenne qui la définit plus largement comme « toute activité consistant à offrir des biens ou des services sur un marché donné $»^{22}$. Il nous semble donc qu'il doit tout autant être qualifié d'opérateur économique.

B. LE CRITÈRE MATÉRIEL : LA SATISFACTION D'UN BESOIN DE COMPENSATION

Il convient de distinguer les deux catégories de contrats créés par la loi Biodiversité, ceux qui ont pour objet la réalisation d'actions compensatoires (1), ou l'achat d'unité de compensation (2.).

1) Le(s) contrat(s) ayant pour objet la réalisation d'actions compensatoires

\section{a) Le contrat principal}

La loi a créé un contrat permettant au maître d'ouvrage de confier la réalisation des actions compensatoires à un " opérateur de compensation » chargé de "mettre en œuvre les mesures de compensation des atteintes à la biodiversité et de les coordonner à long terme » (art. L. 163-1-III C. envir.). Du point de vue du droit de la commande publique, la question se pose de savoir si, compte tenu de son objet et du mode de rémunération de cet opérateur, un tel contrat peut être qualifié de marché public (de travaux, de services

21. V. les arguments de Trébulle F.-G., « Les titres environnementaux », RJE 2011, $\mathrm{n}^{\circ} 2$ p. 203.

22. CJCE 12 sept. 2000, Pavalov, aff. C-180 à 184/98. 
ou de fournitures) ou de concession ce qui a des conséquences sur son régime juridique ( $c f$. infra $2^{\mathrm{e}}$ partie).

Aux termes de l'article 4 de l'ordonnance du 23 juillet 2015, le marché public est " un contrat à titre onéreux » ayant pour objet de « répondre aux besoins de l'acheteur en matière de travaux, de fournitures ou de services ». Cela signifie que la rémunération de son titulaire prend la forme d'un prix, c'est-à-dire d'une somme d'argent versée directement par l'acheteur ou, plus généralement, d'une « contreprestation à laquelle procède l'autorité publique pour obtenir la prestation commandée $»^{23}$. Plus précisément ensuite, le marché de travaux est le contrat par lequel l'acheteur se procure des travaux immobiliers, ayant une emprise au sol, par l'intermédiaire d'un entrepreneur ${ }^{24}$. Le marché de fournitures est le contrat par lequel l'acheteur se procure des produits ou biens mobiliers par l'intermédiaire d'un fournisseur ${ }^{25}$. Le marché de services a " pour objet la réalisation de prestations de services » (art. 5-III).

Le marché public se distingue du contrat de concession par le mode de rémunération de son titulaire. Dans le cadre du second, l'opérateur se rémunère en exploitant l'ouvrage ou le service qui fait l'objet du contrat. Plus précisément, aux termes de l'article 5 de l'ordonnance du 29 janvier 2016, le critère de distinction réside dans la prise d'un risque d'exploitation. L'opérateur se voit «transfér(er) un risque lié à l'exploitation de l'ouvrage ou du service, en contrepartie soit du droit d'exploiter l'ouvrage ou le service qui a fait l'objet du contrat soit ce droit assorti d'un prix $»$.

Pour répondre à la question sus-posée, il convient de s'interroger sur l'objet d'un contrat ayant pour objet de confier la réalisation des actions compensatoires à un opérateur de compensation et sur le mode de rémunération de ce dernier.

23. CJCE 12 juill. 2001, Ordre des architectes de la province de Milan, aff. C-399/98.

24. Il « a pour objet : a) soit l'exécution, soit la conception et l'exécution de travaux dont la liste est publiée au JORF ; b) soit la réalisation, soit la conception et la réalisation, par quelque moyen que ce soit, d'un ouvrage répondant aux exigences fixées par l'acheteur qui exerce une influence déterminante sur sa nature ou sa conception » (art. 5-I).

25. Il a « pour objet l'achat, la prise en crédit-bail, la location ou la location-vente de produits » (art. 5-II). 
Ce contrat de compensation a pour objet de confier à l'opérateur de compensation le soin de "mettre en œuvre les mesures de compensation des atteintes à la biodiversité et de les coordonner à long terme ». Comme l'explique M. Lucas ${ }^{26}$, il s'agit d' « un contrat de gestion de mesures compensatoires », ces dernières prenant la forme de compensations physiques et biologiques sur des milieux (ex. restauration/création de zones humides, défrichage et replantation), lesquelles peuvent être assorties de mesures dites d'accompagnement, qui visent à mettre en œuvre des actions complémentaires de type études patrimoniales, acquisitions de connaissances. Au sens du droit de la commande publique, même si certaines de ces opérations de compensation ont une emprise sur le sol, il est douteux de considérer qu'une telle prestation consiste à fournir des travaux. Il s'agit plus vraisemblablement de prestations de services, ainsi que le suggère une jurisprudence considérant que des travaux d'entretien d'espaces verts constituent des services d'entretien ${ }^{27}$ et que par voie de conséquence, les marchés qui s'y rapportent constituent des marchés publics de services. En pratique néanmoins, on apprend que certains maîtres d'ouvrages se procurent des prestations de compensation par l'intermédiaire d'un marché de travaux dont l'objet est principalement la réalisation d'un travail immobilier. Tel est le cas dans le cadre de l'actuel projet de « cluster » scientifique et technologique Paris-Saclay nécessitant la construction de bureaux, de routes et d'un métro sur des terres agricoles. Ces constructions ayant entraîné la destruction de mares et de zones humides qui contenaient des espèces biologiques rares, elles ont nécessité de recréer des milieux humides artificiels. L'établissement public d'aménagement Paris-Saclay (Epaps) a néanmoins conclu un marché de travaux publics. En effet, la destruction de ces zones humides entraînant un risque d'inondations, il a fait construire un bassin de rétentions d'eaux pluviales. L'avis d'attribution du marché public indique qu'il s'agit de la première destination du bassin. Ce n'est qu' in fine qu'est citée « la réalisation d'une mare de compensation » qualifiée de travaux connexes.

Le Code de l'environnement est en revanche muet quant à la rémunération de l'opérateur de compensation. Selon le directeur de la

26. Lucas M., art. préc., EEI juin 2017.

27. Au sens de l'annexe I.A. de la directive services n ${ }^{\circ} 92 / 50$ et de l'article 379.I du Code des marchés publics dans sa version antérieure à 2001 . 
CDC Biodiversité, $\mathrm{Ph}$. Thievent ${ }^{28}$, un tel contrat « permet au maître d'ouvrage d'externaliser la totalité des risques d'exécution qui sont portés par l'opérateur de compensation ${ }^{29}$. Au sens du droit de la commande publique cependant, on peine à croire qu'il s'agisse d'un transfert de risque assimilable à celui qu'assume un concessionnaire de services. Dans le cadre d'un contrat de compensation, l'opérateur est payé par le maître de l'ouvrage pour réaliser des prestations : s'il ne parvient pas à amortir les investissements financés ou les coûts supportés lors de l'exécution du contrat, cela est dû à une mauvaise estimation des coûts lors de la conclusion du contrat et non à une exposition aux aléas du marché. Dans cette perspective, le contrat de compensation peut être rapproché d'un contrat d'entreprise connu en droit de l'environnement consistant à payer un prestataire pour des services environnementaux ${ }^{30}$. La difficulté est identique : déterminer le juste prix du service ${ }^{31}$, en fonction des investissements consentis pour la protection de la biodiversité.

Nous en concluons que lorsqu'un tel contrat de compensation est conclu entre un pouvoir adjudicateur et un opérateur de compensation, il doit être qualifié de marché public de services.

\section{b) Les contrats accessoires}

A côté de ce contrat, le Code de l'environnement fait allusion à d'autres contrats susceptibles d'être conclus dans la perspective de garantir ce qu'il est appelé « la maîtrise foncière des zones de compensation " lorsque le terrain sur lequel les mesures compensatoires vont être réalisées n'appartient ni au maître d'ouvrage, ni à l'opérateur de compensation (art. L. 163-2) mais, par exemple, à un agriculteur ${ }^{32}$, à une collectivité publique ou à un

\footnotetext{
28. Thievent $\mathrm{Ph}$., «La pratique de la compensation écologique », EEI 2017, ${ }^{\circ}$ 6, dossier 9 ; «La pratique de la CDC Biodiversité : 10 ans d'expérience de gestion de site naturel de compensation », colloque La compensation écologique en questions, Journées scientifiques de l'Université de Nantes, 2 juin 2017.

29. Ce qui n'augure rien du fait que le maître d'ouvrage reste responsable de l'exécution de la compensation devant l'autorité administrative (art. L. 163-1-III al.2 C. envir.).

30. V. Monteiller, thèse préc. $\mathrm{n}^{\circ} 123$ et ss ; Langlais A., « Les paiements pour services environnementaux, une nouvelle forme d'équité environnementale pour les agriculteurs? Réflexions théoriques », Envir. et DD janv. 2013, étude 4.

31. Romi R., «La notion de 'performance écologique' et la rémunération des contrats en matière d'environnement », Dr. Envir. 2009, n 168, p. 3.

32. Etrillard C., « La compensation écologique : une opportunité pour les agriculteurs? », RD rur. 2016, étude 10.
} 
particulier. Il faut alors que le maître de l'ouvrage ou l'opérateur de compensation trouve le(s) terrain(s) disponible(s) pour réaliser la compensation, puis les acquière ou les " maîtrise " grâce à un contrat de location de longue durée et garantisse leur affectation à long terme à cette mission de compensation, une telle recherche étant d'autant plus délicate que la (ou les) parcelle(s) identifiée(s) doit être située(s) à proximité du projet et satisfaire le critère d'équivalence écologique.

La qualification de tels montages contractuels soulève, selon nous, des difficultés.

Lorsque le maître d'ouvrage ou l'opérateur de compensation est une personne privée, ces contrats accessoires semblent être des contrats de droit privé : contrat de vente pour acquérir un terrain ou de bail pour le louer. Il est de surcroît prévu que l'opérateur de compensation, devenu propriétaire, pourrait solliciter le concours des exploitants, en utilisant le bail rural environnemental, dans les conditions prévues par la loi n ${ }^{\circ} 2014-1170$ du 13 octobre 2014 d'avenir pour l'agriculture, l'alimentation et la forêt (art. L. 41127 C. rur.) $)^{33}$. Lorsque le maître d'ouvrage ou l'opérateur de compensation est une personne publique en revanche, ces contrats accessoires peuvent être des contrats administratifs. Imaginons les configurations où le terrain support de la compensation est acheté. S'il est acheté à une personne privée, il s'agit d'un contrat de vente conclu entre une personne publique et une personne privée : il est un contrat de droit privé, sauf s'il comporte une clause exorbitante du droit commun. $\mathrm{Si}$, au contraire, il est acheté à une personne publique qui vendrait une parcelle de son domaine privé (son domaine public étant inaliénable), la qualification du contrat dépend de la personne publique vendeuse. Si l'Etat cède un terrain de son domaine privé, il s'agit d'un contrat administratif par détermination de la loi (art. L. 3231-1 CGPPP $)^{34}$. En revanche, si une autre personne publique cède un terrain de son domaine privé, il ne s'agit d'un contrat administratif qu'exceptionnellement, lorsque le contrat de vente comporte une clause exorbitante ${ }^{35}$.

33. V. l'article de Krajeski D. «Travail du sol, services écosystémiques et bail rural ». 34. CE 8 nov. 1974, Époux Figueras, Rec. 545 ; Cass. 16 oct. 1963, SCI Tour Perret, Bull I p. 439 ; TC 6 juin 2011, Préfet de Seine et Marne, BJCP 2011, p. 481.

35. CE 10 fév. 2016, n 373664, Sté. Générim ; TC 6 juin 2016, Cne d’Aragnouet, n 4051, CMP 2016, comm. 198, Ubaud-Bergeron M. 
Toutes ces qualifications sont toutefois susceptibles d'être discutées si l'on envisage ces contrats relatifs à la maitrise du foncier comme des accessoires du contrat de compensation, faisant partie d'un ensemble contractuel ${ }^{36}$. Un ensemble contractuel désigne plusieurs contrats concourant à une même opération économique mais n'ayant pas à proprement parler le même objet : par exemple, un contrat de cautionnement ou de garantie d'un emprunt, qui serait l'accessoire d'un marché public. En application de cette théorie dite des contrats administratifs par accessoire, des contrats qui, considérés isolément, seraient qualifiés de contrats de droit privé, vont être qualifiés d'administratifs parce qu'ils dépendent de tels contrats. Pour identifier un tel ensemble contractuel indivisible, le juge recherche si la conclusion de l'une des conventions est subordonnée à celle de l'autre ou si les prestations de l'une n'ont d'intérêt ou ne sont possibles qu'au regard de l'autre. Il recherche si les conventions sont imbriquées au point ne plus former qu'une convention unique ${ }^{37}$. Dans le cas d'un contrat de compensation, la question se pose de savoir si, lorsque le contrat principal est un contrat administratif (cf. ci-dessus), le contrat relatif à la maîtrise du foncier ne devrait pas, par application de cette théorie, être qualifié de contrat administratif. Il ne fait en effet guère de doute que la réussite de l'opération de compensation dépend hautement de cette maîtrise du foncier dont elle est indissociable puisqu'à défaut de posséder ou de maîtriser un terrain susceptible d'accueillir la compensation, le maître de l'ouvrage devra se tourner vers la seconde catégorie de contrat proposé par le Code de l'environnement : le contrat d'acquisition d'unités de compensation.

2) Le contrat d'acquisition d'unités de compensation

La loi Biodiversité créé également un contrat portant sur l'acquisition d'unités de compensation dans le cadre d'un site naturel de compensation $^{38}$. Un tel contrat, lorsqu'il est conclu par un pouvoir adjudicateur, devrait être qualifié de marché public de fournitures, contrat conclu à titre onéreux par lequel un acheteur se procure des produits ou biens mobiliers par l'intermédiaire d'un fournisseur (art. 5-II, ordonnance 23 juill. 2015).

36. Mollion G., « La théorie de l'accessoire dans les contrats publics », CMP 2009, étude 10.

37. Concl. Genevois B. sur CE 6 mai 1985, Assoc. Eurolat, n 41589, RFDA 1986, p. 26.

38. Cf. supra Alidor B., « Compensation et services écosystémiques ». 
Certes, la loi ne précise pas ce qu'est une unité de compensation. Elle n'a pas conceptualisé cette notion, ni précisé les conditions d'émission desdites unités, contrairement à ce qu'elle a pu faire pour les quotas de gaz à effet de serre qu'elle a expressément qualifié de « biens meubles exclusivement matérialisés par une inscription au compte de leur détenteur (...) Ils sont négociables, transmissibles par virement de compte à compte et confèrent des droits identiques à leurs détenteurs » (art. L. 229-15 C. envir.). Mais si l'on se fie à la doctrine civiliste ${ }^{39}$, ces unités sont des biens dans la mesure où d'après le législateur, elles doivent être acquises, que dès lors qu'elles ne peuvent être immeubles, elles sont meubles et plus exactement des meubles incorporels puisque ces titres ne portent pas sur la nature elle-même mais sur un service permettant de compenser la sa détérioration prévisible : " le bien et les services demeurent alors dans leur matérialité hors du commerce juridique, mais des titres dématérialisés représentent l'investissement nécessaire à leur production et à leur gestion. Ces titres sont évidemment des biens dans le commerce juridique $»^{40}$. Par suite, ces unités de compensation ne relevant ni de la catégorie des travaux, ni des services, elles sont des fournitures. Les contrats portant sur leur acquisition sont des marchés publics de fournitures.

Ces qualifications soulèvent un certain nombre de difficultés qu'il convient à présent d'envisager.

\section{LE RÉGIME DES CONTRATS DE COMMANDE PUBLIQUE DE COMPENSATION}

Les développements qui vont suivre entendent mettre en évidence quelques difficultés que risque de susciter la combinaison entre les dispositions du Code de l'environnement et du droit de la commande publique, au stade de la passation des contrats de compensation (A) comme à celui de leur exécution $(\mathrm{B})$. Ils ne sont vraisemblablement pas exhaustifs.

39. Trébulle F.G., « Le marché des unités de biodiversité, quelles perspectives ? », EEI $2017, \mathrm{n}^{\circ} 6$, dossier 1 .

40. Martin G.-J., "Les "biens-environnements". Une approche par les catégories juridiques », RIDE économique, 2/2015 (t. XXIX), p. 139. 


\section{A. L'ENCADREMENT DE LEUR PASSATION}

Comme tout contrat de la commande publique, les contrats de compensation « respectent les principes de liberté d'accès à la commande publique, d'égalité de traitement des candidats et de transparence des procédures » (art. $1^{\mathrm{er}}$, ordonnance 23 juill. 2015). Le respect de ces principes implique notamment de lutter contre les conflits d'intérêts ${ }^{41}$, c'est-à-dire de préserver l'impartialité des acheteurs en sanctionnant les procédures dans le cadre desquelles un candidat pourrait être avantagé ou défavorisé en raison des liens qu'ils pourraient entretenir avec lesdits acheteurs. Le secteur de la compensation environnementale n'est pas à l'abri de tels risques de conflits d'intérêts ${ }^{42}$.

Comme tout contrat de la commande publique ensuite, les contrats de compensation environnementale doivent être conclus dans le respect des obligations de publicité et de mise en concurrence prévues par les textes. Cela pourrait soulever trois difficultés. La première, au stade de la définition préalable des besoins de l'acheteur (1), la deuxième, au stade de l'allotissement des prestations objet du marché (2), la troisième au stade du choix de la procédure de dévolution concurrentielle (3).

1) L'obligation de compenser et la définition préalable du besoin de l'acheteur

De prime abord, la définition de l'étendue de l'obligation de compenser les impacts négatifs d'un projet de construction ne devrait pas poser de difficulté puisque les contrats de prestation de service et de gestion doivent reprendre les caractéristiques des mesures de compensations définies par l'arrêté d'autorisation. En réalité, il n'en est rien.

D'un point de vue environnemental, cette définition est complexe. L'objectif d'équivalence écologique que poursuit la compensation

41. Les directives du 26 février 2014 relatives aux marchés publics (2014/24, art. 24) et aux concessions (2014/23, art. 35) contiennent des dispositions explicites en ce sens.

42. Martin G.-J., « Les unités de compensation et l'organisation du marché », présentation dans le cadre d'une journée d'étude consacrée à la compensation organisée par IEJUC, Université Toulouse Capitole, 5 déc. 2016 ; Lucas M., art. préc., Droit et Ville, 2017, à paraître. 
suppose que la conclusion d'un contrat ayant cette finalité soit précédée d'une étude concrète du milieu affecté, destinée à préciser l'étendue de l'obligation de compensation. Cela pose la question de savoir qui va procéder à cette étude. La conclusion d'un accord amiable entre les parties prenantes à l'opération étant exclue, il conviendra de faire appel à un tiers neutre. Cela pose également la question de savoir quand il faut procéder à cette étude. L'obligation de définir ab initio l'étendue de l'obligation de compensation doit être combinée avec la nécessité que les mesures la concrétisant doivent " être effectives pendant toute la durée des atteintes » (art. L. 163-1 C. env.) et avec le fait que les écosystèmes évoluent continuellement.

Or du point de vue du droit de la commande publique, cela a deux conséquences.

D'une part, la difficulté d'identifier a initio les mesures de compensation adéquates va se heurter à l'obligation qui incombe à l'acheteur public, avant le lancement de toute consultation, de déterminer « la nature et l'étendue des besoins à satisfaire » et d'identifier les prestations susceptibles d'y répondre, grâce à des spécifications techniques contenues « dans les documents de marché, définissant les caractéristiques requises d'un matériau, d'un produit ou d'une fourniture » (art. 30 et 31, ordonnance 23 juill. 2015 ; art. 6, décret 25 mars 2016), faute de quoi la procédure de dévolution concurrentielle serait faussée.

D'autre part, la nécessité de recourir aux services d'un tiers pour procéder à une étude du milieu affecté avant la conclusion d'un contrat de compensation risque de nécessiter la conclusion d'un contrat distinct ayant pour objet de la commander.

2) Les opérations de compensation et l'allotissement du marché public

L'exécution d'une obligation de compensation écologique se décompose, le cas échéant, en plusieurs opérations/phases : maîtrise foncière ; réalisation d'études préalables ; réalisation de travaux de génie écologique ; gestion, entretien et suivi des mesures compensatoires ; conclusion de contrats d'assurance en vue d'obtenir une garantie bancaire contre le risque d'inexécution. 
Du point de vue du droit de la commande publique, chacune de ces opérations est susceptible de donner lieu à un contrat spécifique (contrat de bail ou de vente ; marché public de prestations de services ; contrat d'assurance) et/ou à l'attribution d'un lot. En droit des marchés publics en effet, la conclusion d'un marché est soumise à la règle de l'allotissement qui est une technique par laquelle une opération est décomposée en plusieurs lots donnant lieu à la passation d'autant de marchés séparés. En principe, l'allotissement est obligatoire pour tous les acheteurs et ce afin de renforcer la concurrence et de permettre l'accès aux marchés publics des PME. Le nombre de lots est choisi librement par l'acheteur, en fonction des caractéristiques techniques des prestations demandées, de la structure du secteur économique ou de la configuration géographique. Les candidatures et les offres déposées par les opérateurs économiques sont ensuite appréciées lot par lot. Exceptionnellement cependant, cette règle est écartée lorsque l'acheteur est dans l'impossibilité d'identifier des prestations distinctes en raison de l'objet du marché et qu'il n'est pas en mesure d'assurer lui-même " les missions d'organisation, de pilotage et de coordination ou si la dévolution en lots séparés est de nature à restreindre la concurrence ou risque de rendre techniquement difficile ou financièrement plus coûteuse l'exécution des prestations » (art. 32, ordonnance 23 juill. 2015 ; art. 12, décret 25 mars 2016). Elle est également écartée dans les cas strictement énumérés où l'acheteur est autorisé à conclure un marché public global (art. 33 et s., ordonnance 23 juill. 2015). L'un d'entre eux mérite notre attention : le marché public global de performance, associant « l'exploitation ou la maintenance à la réalisation ou à la conception-réalisation de prestations, afin de remplir des objectifs chiffrés de performance définis notamment en termes de niveau d'activité, de qualité de service, d'efficacité énergétique ou d'incidence écologique » (art. 34, ordonnance 23 juill. 2015).

Les mesures de compensation portant sur un processus global de reconstitution d'un écosystème par nature évolutif, les étapes successives ci-dessus décrites nous semblent difficilement dissociables les unes des autres. Dans le but de mener à bien l'opération de compensation, il n'est guère envisageable (ou à tout le moins efficace) de confier à des opérateurs de compensation différents la réalisation des travaux de génie écologique et leur suivi, lequel impliquera une adaptation des mesures en fonction de leurs effets. 
De fait, l'outil contractuel le mieux adapté ${ }^{43}$ apparaît donc comme étant le marché public global de performance.

Il reste néanmoins un obstacle. Dans les régions fortement urbanisées, le maître de l'ouvrage est souvent confronté à la difficulté de faire réaliser son obligation de compensation sur un site d'un seul tenant, remplissant toutes les conditions écologiques requises et, de surcroît, à proximité du lieu de son projet alors même que le Code de l'environnement prévoit que les mesures de compensation doivent être réalisées conformément à l'impératif fixé par le Code de l'environnement (art. L. 163-1-II) ${ }^{44}$. Il est alors contraint de respecter son obligation en diversifiant les sites de compensation et/ou les contrats. Si du point de vue du droit de l'environnement, on peut regretter une perte de cohérence écologique et une difficulté pour contrôler l'effectivité de la compensation, du point de vue du droit de la commande publique, on peut y voir un argument supplémentaire pour imposer un allotissement des prestations en se fondant sur cet élément géographique. Il y aurait alors autant de prestataires que de sites de compensation.

\section{3) Les opérateurs de compensation et la mise en concurrence}

Une fois franchies ces étapes préalables, il convient d'organiser une procédure de dévolution concurrentielle permettant de faire se rencontrer cette demande et l'offre de compensation. A cette fin, il incombe d'abord à l'acheteur de choisir la procédure en fonction de la valeur estimée du besoin.

Lorsque celle-ci est égale ou supérieure aux seuils européens ${ }^{45}$, il y a lieu de mettre en œuvre une des procédures formalisées : 1'appel d'offres, la procédure concurrentielle avec négociation ou le dialogue compétitif. Dans la mesure où ces deux dernières procédures

\footnotetext{
43. Le Bihan Graf Ch., Rosenblieh L., "Les marchés publics de compensation environnementale », EEI 2015, n 6, prat. 6.

44. Quétier F., Quenouille B., Schwoertzig F., Gaucherand S., Lavorel S. et Thiévent P., « Les enjeux de l'équivalence écologique pour la conception et le dimensionnement de mesures compensatoires d'impacts sur la biodiversité et les milieux naturels », Sciences, eaux et territoires, IRSTEA, HS 25 mars 2012.

45. Actuellement : pour les marchés de travaux, $5225000 €$ HT ; pour les marchés de fournitures et de services de l'État et de ses établissements publics, $135000 € \mathrm{HT}$; pour les marchés de fournitures et de services des collectivités territoriales et des établissements de santé, $209000 € \mathrm{HT}$ : art. 42, ordonnance 23 juill. 2015 ; art. 25, décret 25 mars 2016.
} 
permettent d'engager une négociation, leur utilisation est strictement encadrée. Elle est réservée à une série hypothèses listées par l'article 25 de l'ordonnance du 23 juillet 2015. Trois d'entre elles pourraient concerner les marchés de compensation environnementale : celle où « le marché public comporte des prestations de conception », celle où « le marché public ne peut être attribué sans négociation préalable du fait de circonstances particulières liées à sa nature, à sa complexité ou au montage juridique et financier ou en raison des risques qui s'y rattachent » et celle où « le pouvoir adjudicateur n'est pas en mesure de définir les spécifications techniques avec une précision suffisante en se référant à une norme, une évaluation technique européenne, une spécification technique commune ou un référentiel technique ».

Lorsque la valeur estimée du besoin est inférieure aux seuils européens, l'acheteur de compensation peut recourir à « une procédure adaptée dont il détermine librement les modalités en fonction de la nature et des caractéristiques du besoin à satisfaire, du nombre ou de la localisation des opérateurs économiques susceptibles d'y répondre ainsi que des circonstances de l'achat ». Cette procédure l'autorise, le cas échéant, à négocier avec les candidats, à condition de l'avoir indiqué dans les documents de la consultation.

Enfin, dans les seuls cas prévus par l'article 30 du décret du 25 mars 2016, l'acheteur de compensation pourra conclure un marché sans publicité ni mise en concurrence préalable. Outre l'hypothèse où la valeur estimée du besoin est inférieure à 25000 euros HT (art. 30, $8^{\circ}$ ) qui risque fort de ne jamais se rencontrer (si l'on songe qu'une seule unité de compensation sur le site de Cossure a été évaluée à $35000 €^{46}$, soit un montant supérieur au seuil plancher de $25000 €$ ), deux cas sont intéressants. Une attribution de gré à gré est autorisée d'une part, aux termes du $3^{\circ}$, « lorsque les travaux, fournitures ou services ne peuvent être fournis que par un opérateur économique déterminé, pour (...) des raisons techniques » et d'autre part, aux termes du $10^{\circ}$, " pour les marchés publics répondant à un besoin dont la valeur estimée est inférieure aux seuils européens, lorsque la mise en concurrence est impossible ou manifestement inutile en raison notamment de l'objet du marché public ou du faible degré de

46. Thiévent Ph., colloque Nantes, préc., Etrillard C., « La compensation écologique : une opportunité pour les agriculteurs? », préc. 
concurrence dans le secteur considéré ». Ces hypothèses pourraient se rencontrer dans le secteur de la compensation environnementale. En premier lieu, parce que pour l'instant du moins, il y a encore peu d'opérateurs de compensation agissant sur ce marché (CDC Biodiversité a longtemps été seule). En second lieu, parce que la compensation doit être réalisée « en priorité sur le site endommagé ou, en tout état de cause, à proximité de celui-ci » (art. L. 163$1, \mathrm{C}$. envir.), ce qui pourrait conduire à réduire voire à exclure toute concurrence dans la mesure où l'offre de compensation est intimement liée à la maîtrise du foncier destiné à l'accueillir.

Il incombe ensuite à l'acheteur de sélectionner les candidats puis les offres, dans les conditions fixées par l'ordonnance du 23 juillet 2015. Ces dispositions apportent des précisions qui peuvent, nous semble-t-il, contribuer à renforcer le dispositif instauré par le Code de l'environnement.

En effet, la définition de l'opérateur de compensation est suffisamment large pour accueillir de très nombreux opérateurs sans garantie de leurs compétences puisque le législateur a renoncé à soumettre cette qualité à la délivrance d'un agrément ${ }^{47}$. Aux termes de l'article 51 de ladite ordonnance au contraire, un acheteur public peut imposer aux candidats des conditions pour pouvoir participer à la procédure de passation, de nature « à garantir qu'ils disposent de l'aptitude à exercer l'activité professionnelle, de la capacité économique et financière ou des capacités techniques et professionnelles nécessaires à l'exécution du marché public. Ces conditions sont liées et proportionnées à l'objet du marché public ou à ses conditions d'exécution ». Autrement dit, il pourra conditionner une candidature à une véritable aptitude à la restauration écologique.

Par ailleurs, aux termes de l'article 52 de la même ordonnance, le marché doit être attribué à l'opérateur qui a " présenté l'offre économiquement la plus avantageuse sur la base d'un ou plusieurs critères objectifs, précis et liés à l'objet du marché public ou à ses conditions d'exécution » destinés à garantir l'existence d'une véritable concurrence. Ces critères sont précisés par l'article $62 \mathrm{du}$ décret d'application du 25 mars 2016 qui dispose qu'ils peuvent comprendre des « aspects environnementaux » et qu'il peut s'agir,

47. Cf. supra Alidor B. « Compensation et services écosystémiques». 
par exemple, de critères tels que « les performances en matière de protection de l'environnement », « la biodiversité », «l'organisation, les qualifications et l'expérience du personnel assigné à l'exécution du marché public lorsque la qualité du personnel assigné peut avoir une influence significative sur le niveau d'exécution du marché public » et, plus généralement, de critères « justifiés par l'objet du marché public ou ses conditions d'exécution ». Dans cette perspective, il devrait apparaître justifié de restreindre l'accès au marché public en fonction d'un critère géographique qui serait celui de la proximité avec le site endommagé, en fonction de la plus grande performance environnementale ou de la meilleure équivalence écologique.

\section{B. L'ENCADREMENT DE LEUR EXÉCUTION}

L'exécution du contrat de compensation soulève à son tour trois difficultés.

1) Contrôle de l'efficacité des mesures de compensation et modification du contrat de compensation

Quelle que soit la méthode choisie pour mettre en œuvre l'obligation de compensation, le maître d'ouvrage reste seul responsable de l'effectivité des mesures de compensation devant l'autorité administrative qui les lui a imposées (art. L. 163-1-III, al. 2 C. envir.). Tenu à une obligation de résultat, il a l'obligation d'assumer toute défaillance du mécanisme compensatoire qu'il a choisi.

Cette obligation se traduit, au profit de l'autorité administrative compétente pour imposer les mesures de compensation, par un pouvoir de contrôle et de sanction. De fait, elle est, aux termes du Code de l'environnement, dotée de toute une série de pouvoirs destinés à contraindre le maître de l'ouvrage de respecter ses obligations (art. L. 163-4 C. envir.) ${ }^{48}$. Elle peut notamment, lorsque les mesures de compensation sont inopérantes, ordonner des prescriptions complémentaires.

Cette disposition est, du point de vue du droit de la commande publique, problématique. Concrètement, cela signifie que l'obligation

48. Pour plus de détails, $c f$. supra Alidor B. « Compensation et services écosystémiques ». 
de compensation du maître d'ouvrage peut être modifiée au cours de l'exécution du projet lorsque les mesures de compensation choisies ne produisent pas les effets escomptés et, par suite, que le contrat conclu pour mettre en œuvre ces mesures devra être modifié, soit par le biais d'un avenant conclu d'un commun accord entre le maître de l'ouvrage et l'opérateur de compensation, soit par le biais d'un ordre de service modificatif imposé par le maître de l'ouvrage en vertu de son pouvoir de modification unilatérale ${ }^{49}$, si celui-ci est une personne publique et que le contrat conclu est un contrat administratif. Aux termes de l'ordonnance du 23 juillet 2015 (art. 65) et de son décret d'application (art. 139 et 140) cependant, la modification des marchés publics est très encadrée. En toute hypothèse, « les modifications ne peuvent changer la nature globale du marché public ». Elles ne peuvent pas être substantielles, c'està-dire introduire " des conditions qui, si elles avaient été incluses dans la procédure de passation initiale, auraient attiré davantage d'opérateurs économiques ou permis l'admission d'autres opérateurs économiques ou permis le choix d'une offre autre que celle retenue », modifier " l'équilibre économique du marché public en faveur du titulaire d'une manière qui n'était pas prévue dans le marché public initial » ou modifier « considérablement l'objet du marché public ». Ces textes listent ensuite une série d'hypothèses où, exceptionnellement, les modifications sont autorisées. Tel est le cas lorsque celles-ci « ont été prévues dans les documents contractuels initiaux sous la forme de clauses de réexamen, dont des clauses de variation du prix ou d'options claires, précises et sans équivoque $»$. Elles sont alors autorisées, " quel qu'en soit leur montant ». Tel est aussi le cas, dès lors que le montant des modifications n'est pas supérieur à $50 \%$ du montant du marché public initial, lorsque les prestations supplémentaires « sont devenues nécessaires » et à la double condition qu'un changement de titulaire « a) Soit impossible pour des raisons économiques ou techniques tenant notamment à des exigences d'interchangeabilité ou d'interopérabilité avec les équipements, services ou installations existants achetés dans le cadre du marché public initial ; b) Présenterait un inconvénient majeur ou entraînerait une augmentation substantielle des coûts pour l'acheteur » ou lorsque « la modification est rendue nécessaire par des circonstances qu'un acheteur diligent ne pouvait pas prévoir ». Il apparaît ainsi hautement souhaitable que les contrats de

49. CE 2 fév. 1983, UTPUR, Lebon 33 ; RFDA 1984, p. 45, Llorens F. 
compensation contiennent des clauses d'adaptation afin de pouvoir donner un effet utile aux dispositions du Code de l'environnement.

2) Durée de l'obligation de compensation et durée du contrat de compensation

Aux termes de l'article 163-1 du Code de l'environnement, les mesures de compensation doivent « être effectives pendant toute la durée des atteintes $»$. Le temps de la compensation peut donc être très long, voire perpétuel si l'on songe, par exemple, à la disparition d'une zone humide en raison de la réalisation d'une infrastructure urbaine. Théoriquement donc, les contrats de compensation ainsi que ceux permettant la maîtrise foncière doivent permettre d'assurer la pérennité des mesures compensatoires. Le Code de l'environnement est pourtant peu disert sur la durée des contrats de compensation ou sur celle de l'agrément des sites de compensation. Tout au plus apprend-on que lorsque des mesures sont mises en œuvre sur un terrain n'appartenant ni au maître d'ouvrage, ni à l'opérateur de compensation, un contrat conclu avec le propriétaire et, le cas échéant, le locataire ou l'exploitant définit les mesures de compensation « ainsi que leur durée " (art. L. 163-2) et que les opérateurs candidats à l'obtention d'un agrément d'un site naturel de compensation doivent, dans leur dossier, préciser « la durée d'engagement de l'opérateur de compensation pour le site naturel de compensation envisagé (au minimum 30 ans) et les raisons l'ayant conduit à retenir cette durée » (arrêté du 10 avril 2017 fixant la composition du dossier de demande d'agrément d'un site naturel de compensation prévu à l'article D. 163-3 du Code de l'environnement, art. 2). En pratique, il semble que la durée des contrats de gestion des mesures compensatoires soit quant à elle assez variable ${ }^{50}$. Elle est souvent courte ( 5 ans) et renouvelée progressivement, afin de permettre aux cocontractants de tester les pratiques.

Si, du point de vue environnemental, ce dispositif n'est pas de nature à garantir la pérennité de la compensation, dans les hypothèses où il y aurait un changement d'opérateur ou à l'issue des contrats, il pose en outre, du point de vue du droit de la commande publique, une difficulté. Certes, les textes ne fixent pas de durée maximale. Mais ils imposent que la durée du contrat soit déterminée dès la 
mise en concurrence, qu'elle fasse l'objet d'une stipulation expresse (art. 39, ordonnance 23 juill. 2015) et qu'elle soit fixée « en tenant compte de la nature des prestations et de la nécessité d'une remise en concurrence périodique » (art. 16, décret 25 mars 2016). En outre, ils encadrent voire interdisent ${ }^{51}$ le renouvellement des contrats au motif que celui-ci donne naissance à un nouveau contrat qui, le cas échéant, doit faire l'objet d'une nouvelle mise en concurrence ${ }^{52}$. Tel est le cas pour les marchés publics : la reconduction est admise dans son principe mais conditionnée au fait que « la mise en concurrence ait été réalisée en prenant en compte la durée totale du marché, périodes de reconduction comprises », de telle sorte qu'elle apparait alors comme une simple modalité d'exécution du contrat en cours et non comme la conclusion d'un nouveau contrat. En ce sens, l'article 16 du décret du 25 mars 2016 dispose qu'à l'intérieur de la durée fixée par le marché, celui-ci «peut prévoir une ou plusieurs reconductions à condition que ses caractéristiques restent inchangées et que la mise en concurrence ait été réalisée en prenant en compte la durée totale du marché public. Sauf stipulation contraire, la reconduction prévue dans le marché public est tacite et le titulaire ne peut s'y opposer ». Là encore, il apparaît ainsi hautement souhaitable que les contrats de compensation contiennent des clauses de renouvellement dès leur conclusion faute de quoi il faudra relancer une procédure de mise en compétition.

3) Stabilité des acteurs de la compensation, cession et extinction des contrats

L'efficacité des mesures de compensation implique une certaine stabilité des opérateurs, des sites de compensation et du maître de l'ouvrage, débiteur de l'obligation.

Il faut d'abord envisager l'hypothèse où l'opérateur de compensation change au cours de l'exécution du contrat, en raison d'une défaillance de sa part, d'une procédure de liquidation judiciaire, d'un départ à la retraite etc. ou l'hypothèse où le débiteur de l'obligation de compensation disparaît, par exemple à la suite d'une fusion de collectivités. Le droit de l'environnement ne prévoit pas une telle hypothèse. La question se pose pourtant de savoir si l'obligation

51. V. le cas des concessions : art. 34, ordonnance 29 janv. 2016.

52. CE 29 nov. 2000, Cne de Païta, Lebon 573 ; AJDA 2001, p. 219, Richer L. 
de compensation ne devrait pas être expressément transférée au repreneur de l'activité ou de l'ouvrage. Du point de vue du droit de la commande publique cependant, cela se traduit par une cession de contrat, laquelle est fortement encadrée par les textes précédemment évoqués réglementant la modification des contrats. Aux termes de l'article 139 du décret du 25 mars 2016 en effet, la modification du contrat ayant « pour effet de remplacer le titulaire initial par un nouveau titulaire » est qualifiée de modification substantielle : elle est donc interdite. Elle doit faire l'objet d'un nouveau contrat lequel doit, le cas échéant, être mis en concurrence. Elle n'est autorisée qu'à titre exceptionnel, « à la suite d'une opération de restructuration du titulaire initial, à condition que cette cession n'entraîne pas d'autres modifications substantielles et ne soit pas effectuée dans le but de soustraire le marché public aux obligations de publicité et de mise en concurrence » ou « en application d'une clause de réexamen » « claire, précise et sans équivoque » figurant dans le contrat initial. La difficulté vient de ce que la jurisprudence n'a pas précisé le degré de détail d'une telle clause. La question se pose de savoir si l'identité du repreneur doit être précisée dès la signature du contrat initial. Si tel devait être le cas, la cession deviendrait quasiment impossible.

Il faut ensuite envisager l'hypothèse où le gestionnaire du site de compensation se voit retirer son agrément. Celle-ci est envisagée par l'article D. 163-7 du Code de l'environnement : " les maîtres d'ouvrage ayant acquis des unités de compensation sont informés de la mise en œuvre de cette procédure dès la mise en demeure ». Rien n'est en revanche prévu quant aux conséquences de ce retrait pour les maîtres de l'ouvrage qui demeurent débiteurs de l'obligation de compensation, en particulier ceux qui auraient déjà acquis des unités de compensation. Certes, ils pourront se tourner vers les assureurs des gestionnaires de sites de compensation. Mais cela ne résoudra en rien les difficultés qu'ils pourraient rencontrer pour pouvoir matériellement exécuter leur obligation de compensation. La question se pose de savoir si l'autorité administrative pourrait leur imposer de recommencer à zéro les opérations de compensations déjà réalisées. Du point de vue du droit de la commande publique cela impliquerait une nouvelle mise en concurrence.

Enfin et pour conclure, on peut se demander ce qu'il advient des mesures de compensation à l'issue des contrats de compensation et/ou à l'issue de la durée de l'agrément, alors même que les atteintes portées à la biodiversité par certains projets ne sont 
vraisemblablement pas réversibles. On perçoit ici l'intérêt de l'obligation réelle environnementale qui permet d'attacher à un fond des obligations écologiques. Aux termes cependant de l'article L. 132-3 du Code de l'environnement, la durée de cette obligation doit figurer dans le contrat et ne saurait donc être perpétuelle. Sans doute peut-elle durer 99 ans, durée généralement fixée en droit des obligations pour de telles obligations. Mais au terme du contrat, le signataire retrouve sa liberté : le propriétaire récupère le libre usage de ses terrains. A défaut, comme l'a souligné Mme Gaillard, rapporteur de la loi Biodiversité, il y aurait un risque « qu'aucun contractant n'entre dans la logique de la compensation ». 



\title{
Travail du sol, services écosystémiques, et bail rural
}

\author{
Didier KRAJESKI \\ Professeur, Université Toulouse Capitole \\ IEJUC, F-31000
}

\section{INTRODUCTION : LE BAIL RURAL, CONTRAT D’EXPLOITA- TION}

La mise à disposition des terres et des bâtiments nécessaires à l'exploitation entre un propriétaire et une personne susceptible de les exploiter a connu différentes formes juridiques à travers le temps. Depuis la fin de la deuxième guerre mondiale, le législateur français a décidé que ce mode d'exploitation, faire valoir indirect, devait s'incarner dans une forme juridique pour laquelle il limite la part de liberté des parties : le statut des baux ruraux ${ }^{1}$. C'est aujourd'hui un mode d'exploitation du foncier très pratiqué. Envisager, du point de vue des activités agricoles, la question du travail des sols et des services écosystémiques conduit donc nécessairement à envisager la question au regard du bail rural parce que, justement, il occupe une partie substantielle du territoire.

Il est évident qu'une telle convention, en raison de son importance, ne peut être ignorée lorsque les pouvoirs publics souhaitent que l'agriculture contribue à la protection de l'environnement. Les lois venant régulièrement réformer l'agriculture en général, et les baux ruraux en particulier, ont donc progressivement intégré des dispositions manifestant cette préoccupation dans le bail rural même si elle n'en constitue pas l'objet principal qui est, et a toujours été, la bonne exploitation des biens loués. Ainsi, la loi n ${ }^{\circ} 99-574$ du 9 juillet 1999 est venue directement opposer bonne exploitation et protection 
de l'environnement en limitant le droit du bailleur de demander la résiliation du bail, fondée sur la violation des plus classiques obligations du preneur ${ }^{2}$, en raison du développement par le preneur de « pratiques ayant pour objet la préservation de la ressource en eau, de la biodiversité, des paysages, de la qualité des produits, des sols et de l'air, la prévention des risques naturels et la lutte contre l'érosion $»^{3}$. Allant un peu plus loin, la loi $n^{\circ} 2006-11$ vient donner la possibilité au bailleur d'imposer au preneur des pratiques « ayant pour objet la préservation de la ressource en eau, de la biodiversité, des paysages, de la qualité des produits, des sols et de l'air, la prévention des risques naturels et la lutte contre l'érosion, y compris des obligations de maintien d'un taux minimal d'infrastructures écologiques $»$. Autrement dit, le bail rural peut comporter des clauses environnementales. Inséré dans l'article L. 411-27, le texte vient, après avoir consacré l'initiative écologique du preneur, consacrer l'initiative écologique du bailleur. Nous verrons cependant que cette dernière initiative est très strictement encadrée. La loi n 2014-1170 du 13 octobre 2014 a modifié le texte mais elle n'a pas abouti à généraliser cette possibilité. La loi $n^{\circ} 2010-874$ du 10 juillet 2010 en intégrant, à certaines conditions, les produits de la méthanisation à la liste des activités agricoles a automatiquement étendu le champ du bail rural à cette activité. La loi du 13 octobre 2014 a tenu compte de cette extension dans le dispositif prévu pour l'indemnisation des améliorations ${ }^{4}$. Enfin, on peut évoquer la loi $n^{\circ} 2016-1087$ du 8 août 2016 qui articule les nouvelles obligations réelles environnementales et le bail rural ${ }^{5}$.

Dans cet ensemble de normes articulant bail rural et protection de l'environnement, on peut se demander quelles sont les conséquences de l'intégration d'une réflexion appréhendant la relation entre l'humanité et la nature sous l'angle des services écosystémiques. Ils sont définis, dans le présent ouvrage, comme les « avantages

2. On les trouve dans le Code civil, aux articles 1766 et suivants cités par l'article L. 41127. En substance, pour ce qui concerne l'exploitation, ils imposent de ne pas abandonner la culture, ne pas changer la destination des lieux loués, de respecter les clauses du bail, d'exploiter raisonnablement les lieux loués.

3. Art. L. 411-27 C. rur., le texte est cité dans sa version modifiée par la loi n 2006-11du 5 janvier 2006.

4. Art. L. 411-73, 2 C. rur.

5. Art. L. 132-3 C. env. Sur la question : Reboul-Maupin N. et Grimonprez B., 2016. Les obligations réelles environnementales : chronique d'une naissance annoncée, Dalloz, 2074.

$C f$. infra Alidor B. «Compensation et services écosystémiques ». 
socioéconomiques retirés par l'homme de son utilisation durable des fonctions écologiques des écosystèmes $\gg{ }^{6}$. De nombreux auteurs travaillant sur ce thème, quelle que soit leur spécialité, ont émis des réserves sur le caractère réellement novateur du concept et des propositions pouvant en émerger ${ }^{7}$. On peut en particulier se demander s'il aboutit à la mise en œuvre de nouvelles actions concrètes. Malgré les réserves dont il peut faire l'objet, il semble bien, cependant, que ce soit le cas. L'étude agronomique des services écosystémiques semble notamment aboutir à des préconisations précises sur les techniques développées par les exploitants agricoles. Ces techniques pourraient permettre de développer le potentiel de services rendus par un écosystème non seulement à l'exploitant ou au propriétaire des biens exploités, mais aussi à des tiers. Il en va ainsi, par exemple, de l'agriculture de conservation. Elle se développe autour de trois principes indissociables ${ }^{8}$ : rotation culturale allongée et diversifiée, couvert organique permanent des sols, perturbation minimale du sol. Elle procure des avantages à l'exploitant agricole qui produit en utilisant moins d'intrants. Elle en procure au propriétaire qui voit son sol retrouver ou augmenter ses qualités productives. Elle en procure enfin aux tiers du fait, au moins, de l'économie de produits réalisée.

On retiendra de l'exemple de l'agriculture de conservation que les avantages retirés des services écosystémiques nécessitent une modification des pratiques agricoles et l'adoption de nouvelles méthodes de production, c'est-à-dire une contribution active de l'exploitant agricole dans sa façon même de produire. Elle met donc directement en question le bail rural dans sa façon d'organiser l'exploitation des biens loués : le preneur peut-il y procéder à sa convenance? Le bailleur peut-il l'y contraindre ? Peut-on retirer un profit de ces pratiques, indépendamment de celui qu'elles impliquent directement, ou au moins une indemnisation?

Il convient ici de le rappeler, le bail rural, tel qu'il a été conçu après la deuxième guerre mondiale, s'affranchit du rapport classique dont on trouve encore des manifestations dans le Code civil : à un bail

\footnotetext{
6. V. sur ce point le glossaire.

7. Fèvre M., 2016. Les services écologiques et le droit, Thèse, Université Côte d'Azur, p. 15 et ss. - Doussan I., " Brève histoire de l'intégration de la notion de service écosystémique en droit », supra.

8. V. sur ce point supra Chabert A. et Sarthou J.-P. «Agriculture de conservation des sols et services écosystémiques ».
} 
caractérisé par une volonté de préserver le bien du propriétaire a été substitué un bail dans lequel on se soucie de la stabilité du preneur et de sa liberté d'exploitation'. S'il peut être sanctionné notamment pour n'avoir pas correctement exploité les biens loués ${ }^{10}$, il doit être indemnisé des améliorations qu'il a pu procurer ${ }^{11}$. Il ne faut pas s'y tromper, une préoccupation reste au cœur de la réglementation du statut des baux ruraux : la nécessité d'exploiter les biens loués, en particulier le sol, pour préserver leur capacité à produire ${ }^{12}$. Ce qui a changé entre le Code civil et le Code rural et de la pêche maritime, c'est l'augmentation des prérogatives du preneur, et la diminution corrélative de celles du bailleur, pour présider au sort des biens loués pendant la durée de la location.

C'est au regard de ces principes singularisant le bail rural que doivent être envisagées les pratiques favorisant le développement des services écosystémiques. Dans l'ordre logique, elles doivent conduire à aborder deux questions : comment intégrer ces pratiques dans la relation contractuelle que génère le bail rural ? Quelles conséquences découlent de l'intégration de ces pratiques?

\section{LA POSSIBILITÉ D'INTÉGRER DANS LA RELATION CONTRACTUELLE LE RECOURS AUX PRATIQUES AGRI- COLES FAVORISANT LE DÉVELOPPEMENT DE SERVICES ÉCOSYSTÉMIQUES}

La prise en compte progressive des préoccupations écologiques dans le bail rural a mis en évidence plusieurs éléments qui peuvent ici être déterminants. D'abord, il faut envisager la question du point de vue de chacune des parties. En effet, si l'on est parti d'une possibilité pour le preneur de recourir à ces pratiques, on s'est aussi interrogé sur le droit du bailleur d'imposer ce type de pratiques au preneur. Ensuite, ce qui singularise les pratiques en faveur des services écosystémiques est le fait qu'elles associent de façon indissociable méthode

\footnotetext{
9. Dupeyron C., 1994. Droit agraire, Economica, n 543 s. - Krajeski D., 2016. Droit rural, Defrénois, $\mathrm{n}^{\circ} 147$.

10. Art. L. 411-31-I, 2 C. rur. : il faut qu'il soit l'auteur d'agissements de nature à compromettre la bonne exploitation, ce dont le bailleur doit apporter la preuve.

11. Art. L. 411-69 s. C. rur.

12. Sur cette question : Desrousseau M., 2016. La protection juridique de la qualité des sols, LGDJ, n ${ }^{\circ} 76$ et ss.
} 
d'exploitation agricole et action en faveur de l'environnement. Ce n'est pas simplement une façon de produire avec des égards qui est envisagée, c'est une nouvelle façon d'associer l'écosystème à la production agricole. Ces différents éléments ont nécessairement des conséquences sur la façon d'intégrer les pratiques considérées dans le bail rural.

\section{A. LA POSSIBILITÉ POUR LE PRENEUR DE RECOURIR AUX PRATIQUES AGRICOLES FAVORISANT LE DÉVELOPPEMENT DE SERVICES ÉCOSYSTÉMIQUES}

Lorsque l'on envisage la possibilité pour le preneur de recourir aux pratiques agricoles favorisant le recours aux services écosystémiques, on se trouve tout de suite confronté à la double nature de ces pratiques (protection de l'environnement et méthode de production agricole). Si l'article L. 411-27 du Code rural et de la pêche maritime semble être le point d'entrée évident dans le contrat, une lecture plus attentive semble contredire cette idée. A priori, les pratiques considérées peuvent aisément entrer dans le champ large (et élargi) du texte (préservation de la ressource en eau, de la biodiversité, des paysages, de la qualité des produits, des sols et de l'air, la prévention des risques naturels et la lutte contre l'érosion). Il convient de rappeler que ce texte se borne à protéger le preneur contre une sanction (la résiliation). Il ne préjuge pas de la façon de recourir en cours de bail à ces pratiques surtout quand elles modifient sa façon de faire de l'agriculture, ce qui est le cas de celles que nous évoquons.

Lorsqu'est en question la façon de produire du preneur et l'étendue de sa liberté sur ce point, il semble plus naturel de se référer à l'article L. 411-29 du Code rural et de la pêche maritime qui a l'avantage de traiter directement la question du changement des pratiques culturales. Il conduit à une réponse claire à la question de savoir si le preneur peut adopter les pratiques considérées, au besoin malgré l'opposition du bailleur. Pour de nombreux auteurs, ce texte caractérise la liberté d'exploitation du preneur. Pour ce qui nous intéresse, il donne la possibilité au preneur de changer ses pratiques de production.

La procédure prévue suit une logique assez courante en droit rural, et pour tout dire, raisonnable. Le texte privilégie dans un premier temps l'accord des parties et prévoit, dans un second temps, un arbitrage en cas de désaccord. La disposition se concentre sur 
les moyens culturaux mis en œuvre par le preneur plus que sur la dimension protectrice de l'environnement de son projet qui n'est pas évoquée. C'est rappeler que le bail rural est avant tout un contrat d'exploitation. Ce qui est pris en considération ici c'est l'aptitude des nouvelles méthodes de production à améliorer les conditions de l'exploitation. Le preneur devra être en mesure d'en apporter la preuve. De ce point de vue, la possibilité d'évaluer ces pratiques pourra être déterminante de leur mise en œuvre en matière de baux ruraux ${ }^{13}$.

Le preneur pourra, en effet, être amené à en discuter devant le tribunal paritaire des baux ruraux si le bailleur, informé des modifications à opérer, saisit la juridiction parce qu'il estime que s'ensuivra une dégradation du fonds. Il paraît donc difficile pour le preneur de s'aventurer vers des pratiques qui n'ont pas fait leurs preuves.

Le preneur à bail rural semble posséder un moyen de recourir aux pratiques considérées pour autant qu'il puisse en démontrer l'efficacité. Le bailleur peut-il les lui imposer?

B. LA POSSIBILITÉ POUR LE BAILLEUR D'IMPOSER LES PRATIQUES AGRICOLES FAVORISANT LE DÉVELOPPEMENT DE SERVICES ÉCOSYSTÉMIQUES

En raison du lien étroit entre ces pratiques et les méthodes d'exploitation, la question ne semble pas devoir se distinguer de celle consistant à déterminer si le bailleur peut imposer au preneur la façon d'exploiter. Les développements sur la liberté d'exploitation du preneur sont de nature à apporter une réponse claire : le bailleur n'est pas (ou plus ${ }^{14}$ ) maître de la façon dont les biens sont exploités. Il ne peut donc imposer au preneur des méthodes de production. Cependant, la double nature des pratiques considérées semble devoir conduire à amoindrir l'affirmation. Autrement dit, la dimension de protection de l'environnement de ces pratiques pourrait conduire

13. Sur la question de l'évaluation : intervention de Thérond O. « Dynamiques d'opérationnalisation du cadre conceptuel des services écosystémiques : application aux écosystèmes agricoles.», atelier du 3 mai 2016, ATS T2SEC, IEJUC, Les services écosystémiques rendus par les écosystèmes agricoles. Une contribution au programme EFESE, résumé de l'étude réalisée par l'INRA, oct. 2017.

14. C'est bien ce que sous-entend l'article L. 411-29 du Code rural et de la pêche maritime lorsqu'il évoque l'article $1766 \mathrm{du}$ Code civil pour en écarter l'application. Ce dernier fixe les obligations du preneur, que nous avons rappelées, dans l'ancienne conception du bail rural. 
le bailleur à les imposer plus facilement. Si tel devait être notre constat, le paradoxe ne serait pas des moindres. Nous allons voir, cependant, qu'en l'état actuel des textes les possibilités sont assez réduites. Le bailleur peut, théoriquement, en effet utiliser deux outils pour intégrer des pratiques en faveur de l'environnement dans un bail rural : les clauses environnementales et l'obligation réelle environnementale.

L'insertion de clauses environnementales dans un bail rural intervient dans un cadre étroit, nous l'avons souligné. L'annonce faite dans l'article L. 411-27 paraît tout d'abord large, elle répond en ce sens à la mesure adoptée en faveur du preneur dans le même texte : «Des clauses visant au respect par le preneur de pratiques ayant pour objet la préservation de la ressource en eau, de la biodiversité, des paysages, de la qualité des produits, des sols et de l'air, la prévention des risques naturels et la lutte contre l'érosion, y compris des obligations de maintien d'un taux minimal d'infrastructures écologiques, peuvent être incluses dans les baux ». Cependant, les restrictions interviennent rapidement.

D'abord, cette insertion n'est possible que dans trois cas précisément déterminés par le texte ${ }^{15}$. Ensuite, cette insertion ne peut viser que les pratiques qui sont énumérées dans 1'article R. 411-9-11-1 du Code rural et de la pêche maritime ${ }^{16}$. Les pratiques que le bailleur entend

15. A savoir : - pour garantir, sur la ou les parcelles mises à bail, le maintien de ces pratiques ou infrastructures ; - lorsque le bailleur est une personne morale de droit public, une association agréée de protection de l'environnement, une personne morale agréée «entreprise solidaire», une fondation reconnue d'utilité publique ou un fonds de dotation ; - pour les parcelles situées dans certains espaces mentionnés par l'article L. 411-27.

16. A savoir : le non-retournement des prairies ; la création, le maintien et les modalités de gestion des surfaces en herbe ; les modalités de récolte ; l'ouverture d'un milieu embroussaillé et le maintien de l'ouverture d'un milieu menacé par l'embroussaillement ; la mise en défens de parcelles ou de parties de parcelle ; la limitation ou l'interdiction des apports en fertilisants ; la limitation ou l'interdiction des produits phytosanitaires ; la couverture végétale du sol périodique ou permanente pour les cultures annuelles ou les cultures pérennes ; l'implantation, le maintien et les modalités d'entretien de couverts spécifiques à vocation environnementale ; l'interdiction de l'irrigation, du drainage et de toutes formes d'assainissement; les modalités de submersion des parcelles et de gestion des niveaux d'eau; la diversification de l'assolement; la création, le maintien et les modalités d'entretien de haies, talus, bosquets, arbres isolés, arbres alignés, bandes tampons le long des cours d'eau ou le long des forêts, mares, fossés, terrasses, murets ; les techniques de travail du sol; la conduite de cultures ou d'élevage suivant le cahier des charges de l'agriculture biologique ; les pratiques associant agriculture et forêt, notamment l'agroforesterie. 
voir développer, qui peuvent être une combinaison des différentes possibilités, doivent entrer dans cette liste s'il veut pouvoir les imposer au preneur avec lequel il va conclure un bail rural. Cela ne pose pas de difficultés pour l'agriculture de conservation.

S'il avait été envisagé de généraliser le recours au bail environnemental lors du vote de la loi du 13 octobre 2014, l'idée a été abandonnée en raison des incohérences pouvant résulter d'une multiplication désordonnée des pratiques. La réglementation a tenu compte de ce risque. L'article R. 411-9-11-3 prévoit en effet que si les pratiques sont mises en œuvre en raison de la localisation des parcelles, elles doivent être conformes au document de gestion officiel de l'espace protégé considéré. Dans l'hypothèse où le recours aux clauses environnementales est possible à raison de la qualité du bailleur, il convient de choisir les pratiques qui répondent aux préoccupations environnementales du lieu de situation du bien loué.

Si le recours au bail environnemental apparaît un peu décevant en raison de ses faibles perspectives de diffusion, le recours à l'obligation réelle environnementale l'est encore plus. Selon la loi $\mathrm{n}^{\circ}$ 2016-1087 du 8 août 2016, il s'agit de donner aux propriétaires de biens immobiliers la possibilité de " conclure un contrat avec une collectivité publique, un établissement public ou une personne morale de droit privé agissant pour la protection de l'environnement en vue de faire naître à leur charge, ainsi qu'à la charge des propriétaires ultérieurs du bien, les obligations réelles que bon leur semble, dès lors que de telles obligations ont pour finalité le maintien, la conservation, la gestion ou la restauration d'éléments de la biodiversité ou de fonctions écologiques $»^{17}$. On le voit, dans les conditions prévues par le texte, et pour un champ qui n'intègre pas explicitement le développement des services écosystémiques, il s'agit pour un propriétaire de s'obliger lui-même ainsi que ses successeurs. Il ne s'agit pas d'imposer directement à l'exploitant la charge créée. Plus encore, le texte prévoit une protection du preneur en place : le propriétaire ne peut mettre en œuvre l'obligation réelle environnementale sans l'accord préalable du preneur. Son silence gardé pendant deux mois vaut acceptation et son refus doit être motivé. Quoi qu'il en soit, même acceptée, l'obligation ne s'impose 
pas au preneur, seul le propriétaire en reste tenu. Dans un bail rural ${ }^{18}$, la seule façon d'imposer les comportements qu'elle implique serait de mettre en place... des clauses environnementales dans le bail, c'est-à-dire revenir au mécanisme que nous venons d'évoquer et qui semble bien le seul outil vraiment efficace en la matière. Encore fautil que l'engagement pris par le propriétaire entre dans la liste que nous avons évoquée et que l'on se trouve dans l'une des hypothèses où de telles clauses peuvent être stipulées. En cas de conclusion d'un bail rural postérieurement à la souscription de l'obligation réelle environnementale, la même solution (si c'en est une) sera utilisée car la difficulté est la même : l'obligation ne lie, par sa seule existence, que le propriétaire du bien auquel elle s'applique.

En l'état actuel des textes, il n'est pas surprenant de constater que le preneur pourra assez aisément s'adonner à des pratiques favorisant le développement de services écosystémiques, même en cas de désaccord du bailleur. La difficulté semble, pour lui, résider dans la preuve de l'efficacité de ces pratiques sur les conditions de l'exploitation. Le bailleur ne pourra que difficilement soumettre son preneur à ces pratiques. Dans tous les cas, il reste à se demander quelles conséquences peuvent découler de l'intégration de ces pratiques au bail rural.

II. LES CONSÉQUENCES DE L'INTÉGRATION DE PRATIQUES FAVORISANT LE DÉVELOPPEMENT DE SERVICES ÉCOSYSTÉMIQUES DANS LA RELATION CONTRACTUELLE

Indépendamment des avantages que procurent les services écosystémiques, il s'agit ici de déterminer les conséquences que l'intégration de nouvelles pratiques pourrait avoir sur la relation contractuelle. Ces conséquences diffèrent en fonction de la personne, preneur ou bailleur, qui est à l'origine d'un changement de pratiques.

\section{A. Les CONSÉQuences D'Un ChANGEMENT DE PRATIQues À L'INITIATIVE DU PRENEUR}

Dans la relation qui unit le preneur au bailleur, le changement de méthodes de production doit être pris en compte du point de vue

18. Pour une solution externe : Reboul-Maupin N. et Grimonprez B., article précité. 
des règlements qui ont lieu à la fin d'un bail. En effet, il constitue certainement une amélioration des lieux loués qui peut entrainer une indemnisation.

En matière de baux ruraux, l'indemnisation des améliorations ne s'envisage qu'au moment de la rupture quelle qu'en soit la cause. La procédure d'indemnisation de ces améliorations est très soigneusement réglementée. En principe ne sont indemnisées que les améliorations présentant une utilité certaine pour l'exploitation et ayant été régulièrement effectuées. Cela signifie que le preneur a respecté la procédure prévue par l'article L. 411-73 du Code rural et la pêche maritime. Cependant, concernant l'indemnisation des améliorations que nous évoquons, il faut tenir compte de leur origine. En effet, l'adoption de ces pratiques relèvent de l'article L. 411-29. Or, selon ce texte, le preneur qui modifie les méthodes culturales sur ce fondement est privé, sauf accord du bailleur, du droit d'obtenir une indemnisation pour les améliorations procurées aux biens loués.

Cet accord du bailleur ne signifie d'ailleurs pas que les parties peuvent librement fixer le montant de l'indemnité due au bailleur. En principe, les textes fixant le calcul de l'indemnité d'amélioration sont d'ordre public ${ }^{19}$, mais la jurisprudence semble infléchir ce principe au moins lorsqu'il aboutit à un résultat favorable au preneur ${ }^{20}$. La conversion à l'agriculture de conservation semble devoir être intégrée, assez naturellement, aux pratiques ayant pour objet d'améliorer la productivité des sols. Il est prévu à l'article L. 411-73, 3 , que « en ce qui concerne les travaux de transformation du sol en vue de sa mise en culture ou d'un changement de culture entraînant une augmentation du potentiel de production du terrain de plus de $20 \%$, les améliorations culturales ainsi que les améliorations foncières mentionnées à l'article L. 411-28, l'indemnité est égale à la somme que coûteraient, à l'expiration du bail, les travaux faits par le preneur dont l'effet est susceptible de se prolonger après son départ, déduction faite de l'amortissement dont la durée ne peut excéder dix-huit ans. Le montant de l'indemnité peut être fixé par comparaison entre l'état du fonds lors de l'entrée du preneur dans les lieux et cet état lors de sa sortie ou au moyen d'une expertise. En ce cas, l'expert peut utiliser toute méthode lui permettant d'évaluer, 
avec précision, le montant de l'indemnité due au preneur sortant ». On le voit les conditions de l'indemnisation sont très strictement fixées !

Il est à noter que les textes prévoient une indemnité au profit du bailleur s'il devait résulter de l'intervention du preneur une dégradation des lieux loués ${ }^{21}$.

\section{B. Les CONSÉQueNCES D'Un CHANGEMENT DE PRATIQUES À L'INITIATIVE DU BAILLEUR}

Si la mise en œuvre des pratiques favorisant les services écosystémiques passe par l'insertion de clauses environnementales dans le bail, cela produit plusieurs effets sur la relation des parties. Il y a un aménagement au statut classique des baux ruraux sur deux points.

En premier lieu, le loyer dû par le preneur est minoré. En principe, ce loyer est fixé entre des maxima et des minima prévus par un arrêté préfectoral ${ }^{22}$. Le texte prévoit des circonstances justifiant la majoration ou la minoration du loyer. Il en va ainsi de l'insertion des clauses environnementales : dans ce cas les minima ne s'appliquent pas pour la détermination du loyer.

En second lieu, le preneur s'expose plus facilement à la résiliation du bail ou à son non-renouvellement ${ }^{23}$. Une hypothèse de résiliation du bail spécifique a été créée dans l'article L. 411-31 du Code rural et de la pêche maritime. Cet article récapitule les manquements du preneur qui pourraient justifier une demande en justice en résiliation du bail. Il est prévu que le simple non-respect des clauses environnementales peut fonder cette résiliation. A cet égard l'article R. 411-9-11-4 du Code rural et de la pêche maritime prévoit que le bail organise les conditions dans lesquelles le bailleur s'assure annuellement du respect par le preneur des pratiques convenues. Il en résulte une fragilisation du bail car, en principe, il faudrait démontrer des agissements du preneur de nature à compromettre la bonne exploitation du fonds. En théorie, le preneur peut parfaitement exploiter les biens tout en ne respectant pas ces engagements

21. Art. L. 411-72 C. rur.

22. Art. L. 411-11 C. rur.

23. Les causes de résiliation peuvent être invoquées à l'appui d'un refus de renouvellement du bail rural : art. L. 411-53 C. rur. 
spécifiques. On peut cependant se demander, s'agissant de pratiques favorisant le développement des services écosystémiques, si la distinction a réellement une utilité tant sont liés, en la matière, l'action en faveur de l'environnement et l'exploitation des terres puisque l'exploitation bénéficie de cette action. Ce lien indissoluble est peut-être la meilleure garantie d'exécution de ces engagements. 
Service écosystémique et droit de la responsabilité 



\title{
L'incidence des services écosystémiques en droit de la responsabilité civile
}

\author{
Séverin JEAN \\ Maître de conférences, Université Toulouse Capitole \\ IEJUC, F-31000
}

L'expression "services écologiques $\|^{1}$ est apparue pour la première fois en droit de la responsabilité à travers la directive du 21 avril 2004, relative à la responsabilité environnementale ${ }^{2}$, transposée ${ }^{3}$ aujourd'hui aux articles L. 160-1 et suivants du Code de 1'environnement. En effet, 1'article L. 161-1-I-4 dispose que « constituent des dommages causés à l'environnement au sens du présent titre les détériorations directes ou indirectes mesurables de l'environnement qui : (...) affectent les services écologiques (...) ». L'insertion de cette expression au sein de la responsabilité environnementale n'est guère étonnante dans la mesure où, comme le souligne Madame Mélodie Fèvre dans sa thèse ${ }^{4}$, on assiste aujourd'hui à une crise des fonctionnalités des écosystèmes et partant, à une crise des services rendus. Aussi, la responsabilité - environnementale - se présentait comme l'outil juridique efficace, pour ne pas dire « magique », pour assurer la réparation des atteintes à l'environnement et, plus spécialement, de celles portées aux services écosystémiques.

1. Ou « services écosystémiques » dans la mesure où le présent ouvrage les traite comme des synonymes. En ce sens, voir Glossaire.

2. Directive 2005/35/CE du Parlement Européen et du Conseil du 21 avril 2004 sur la responsabilité environnementale en ce qui concerne la prévention et la réparation des dommages environnementaux.

3. Par la loi $\mathrm{n}^{\circ} 2008-757$ du 1 er août 2008 relative à la responsabilité environnementale et à diverses dispositions d'adaptation au droit communautaire dans le domaine de l'environnement.

4. Fèvre M., Les services écologiques et le droit. Une approche juridique des systèmes complexes, Thèse, 2016, p. 52. 
Pourtant, au-delà du faible emploi de la responsabilité environnementale sur le terrain judiciaire ${ }^{5}$, c'est surtout son champ d'application restreint qui peut expliquer l'absence de succès de la responsabilité environnementale. Assurément, en limitant cette responsabilité à certaines ressources ${ }^{6}$, en l'imputant aux seuls exploitants dans le cadre d'une activité professionnelle ${ }^{7}$, en ne retenant que les préjudices écologiques purs $^{8}$, en n'envisageant que les faits générateurs du dommage intervenus avant le 30 avril 2007, en excluant les dommages à l'environnement survenus dans certaines circonstances ${ }^{9}$, il n'est pas étonnant que cette responsabilité environnementale - spéciale - ne soit pas parvenue, du moins pleinement, à satisfaire les objectifs poursuivis au titre desquels figure notamment la remise en état initial des services écologiques ${ }^{10}$. Toutefois, il ne faudrait pas dépeindre un tableau aussi maussade car la responsabilité environnementale dispose de quelques atouts : une responsabilité quasiment sans faute ${ }^{11}$, une réparation exclusivement en nature conforme au but poursuivi, l'absence de gravité exigée au titre des atteintes aux services écosystémiques ${ }^{12}$. Autrement dit, il ne s'agit pas de souhaiter la disparition de ce régime spécial de responsabilité environnementale mais de dire qu'il est insuffisant. Cette carence a sans doute conduit le législateur à réagir en consacrant récemment d'une part, la réparation du préjudice écologique au sein du Code civil ${ }^{13}$ et d'autre part, en ajoutant, pour consolider l'édifice, une action de groupe en matière environnementale ${ }^{14}$.

\footnotetext{
5. Doit-on peut-être citer un arrêt récent rendu par la Cour de cassation qui refusa, au visa de l'article L. 162-1 du Code l'environnement, de retenir la responsabilité environnementale d'une société organisant des randonnées motorisées au motif que la cour d'appel n'avait pas recherché « si l'activité professionnelle de la société (...) faisait partie de celles permettant de retenir une réparation du dommage causé à l'environnement » (Cass. $3^{\text {ème }}$ civ., 29 sep. 2016, $\left.\mathrm{n}^{\circ} 15-20048\right)$.

6. Cf. art. L. $161-1-\mathrm{I}-4^{\circ} \mathrm{C}$. env.

7. Cf. art. L. $162-1$ C. env.

8. Cf. art. L. $162-2$ C. env.

9. Cf. art. L. 161-2 C. env.

10. $C f$. art. L. 161-9 C. env.

11. $C f$. art. L. $162-1-1^{\circ}$ du Code précité. Cela étant le $2^{\circ}$ du même article exige une faute ou une négligence de l'exploitant.

12. $C f$. art. L. $161-1-\mathrm{I}-4^{\circ} \mathrm{C}$. env.

13. $C f$. les articles 1246 et s. du Code civil issus de la loi n ${ }^{\circ} 2016-1087$ du 8 août 2016 pour la reconquête de la biodiversité, de la nature et des paysages.

14. Cf. spécifiquement l'article L. 142-3-1 du Code de l'environnement issu de la loi $\mathrm{n}^{\circ}$ 2016-1547 du 18 novembre 2016 de modernisation de la justice du XXI ${ }^{\text {ème }}$ siècle.
} 
Si l'expression " services écologiques » ou "services écosystémiques »n'est pas expressément employée dans la nouvelle action de groupe, il y a tout lieu de penser que cette action joue pleinement en la matière puisque 1'article L. 142-3-1-II du Code de l'environnement traite de tous les préjudices résultant d'un dommage dans les domaines visés par l'article L. 142-2 du même Code. Or, étant donné qu'il est question de nature, d'environnement, il est certain que les atteintes aux services écologiques entrent dans le champ d'application de l'action de groupe nouvellement créée. Les choses sont en revanche beaucoup plus claires quand il s'agit de s'intéresser à la responsabilité civile délictuelle tendant à la réparation du préjudice écologique du Code civil. En effet, l'article 1247 du Code civil dispose qu' « est réparable, (...) le préjudice écologique consistant en une atteinte non négligeable (...) aux bénéfices collectifs tirés par l'homme de l'environnement ». Bien que l'article 1247 du Code civil n'use pas de l'expression « services écologiques » ou « services écosystémiques », les bénéfices collectifs tirés par l'homme de l'environnement constituent assurément un synonyme dans la mesure où les bénéfices correspondent aux services, aux bienfaits que retire l'homme de l'écosystème entendu plus maladroitement au sein du Code civil par la référence à l'environnement. L'orientation 《 anthropocentrée ${ }^{15}$ du texte ne laisse subsister aucun doute : les services écosystémiques entrent dans le champ de la responsabilité civile délictuelle des articles 1246 et suivants du Code civil. Aussi, la question n'est plus de savoir si les services écologiques sont prévus par l'article 1247 du Code précité, mais de se demander si l'insertion du préjudice écologique, constitué notamment par une atteinte aux dits services, influence la responsabilité civile délictuelle.

La réponse pour le signataire est évidente : assurément. Assurément, parce que l'influence des services écosystémiques se retrouve non seulement au stade des conditions de mise en œuvre de la responsabilité civile (I) mais également au stade du régime de responsabilité civile lui-même (II). 


\section{L'INCIDENCE DES SERVICES ÉCOSYSTÉMIQUES SUR LES CONDITIONS DE MISE EN EEUVRE DE LA RESPONSABILITÉ CIVILE}

La mise en œuvre de la responsabilité civile exige trois conditions cumulatives : un fait générateur, un dommage et un lien de causalité entre les deux premiers éléments. Or, l'insertion des services écosystémiques au sein de la responsabilité civile est l'occasion de repenser au moins deux de ces conditions fondamentales. D'une part, parce qu'un préjudice exige nécessairement un dommage tandis que ce dernier ne conduit pas assurément à un préjudice, il convient de savoir si les services écosystémiques - les bénéfices collectifs tirés par l'homme de l'environnement - forment un dommage ou un préjudice. Autrement dit, les services écosystémiques sont l'occasion d'affirmer, une fois de plus, la distinction entre le dommage et le préjudice (A). D'autre part, la responsabilité civile a toujours été pensée à partir des faits générateurs : faits personnels, faits des choses, faits d'autrui. Toutefois, il est frappant de constater que la responsabilité civile, en matière de services écosystémiques, se moque du fait générateur ; seule comptant la nature du dommage. Dès lors, cette indifférence interroge à nouveau sur l'opportunité de construire des régimes de responsabilité civile non plus à partir des faits générateurs mais à partir de la nature des dommages. Dès lors, les services écosystémiques sont l'occasion de repenser, peut-être à son détriment, la responsabilité civile elle-même (B).

\section{A. LES SERVICES ÉCOSYSTÉMIQUES AU SERVICE DE LA DISTINCTION ENTRE LE DOMMAGE ET LE PRÉJUDICE}

La rédaction de l'article 1247 du Code civil est l'occasion de mettre en évidence la distinction opérée par une partie de la doctrine entre le dommage et le préjudice ${ }^{16}$; le dommage constituant le seuil de la lésion, le préjudice établissant les conséquences - patrimoniales ou extrapatrimoniales - de la lésion. En effet, l'article 1247 du

\footnotetext{
16. « Le dommage, désigne, à proprement parler, la lésion subie, qui s'apprécie au siège de la lésion, tandis que le préjudice, qui est la conséquence de la lésion, apparaît comme l'effet ou la suite du dommage : une atteinte à l'intégrité physique, c'est-à-dire un dommage corporel, peut ainsi engendrer des préjudices patrimoniaux (...) et des préjudices extrapatrimoniaux (...) », Le Tourneau Ph. et al, Droit de la responsabilité civile et des contrats, Régimes d'indemnisation, Dalloz-action, 2017-2018, $\mathrm{n}^{\circ} 1304$. Pour une démonstration plus complète, voir les $n^{\circ} 1304$ et ss. du même ouvrage.
} 
Code précité dispose qu' « est réparable (...) le préjudice écologique consistant en une atteinte non négligeable aux éléments ou aux fonctions des écosystèmes ou aux bénéfices collectifs tirés par l'homme de l'environnement ». Il faudrait alors comprendre que l'atteinte aux éléments des écosystèmes tout comme les atteintes aux fonctions des écosystèmes ou encore aux bénéfices collectifs tirés par l'homme de l'environnement constituent des préjudices écologiques. Pourtant, à bien y regarder, le législateur confond le dommage et le préjudice en mettant sur le même plan l'un et l'autre.

Le préjudice écologique serait d'abord constitué par l'atteinte aux éléments des écosystèmes. Pourtant un écosystème est défini comme « un complexe dynamique formé de communautés de plantes, d'animaux et de micro-organismes et de leur environnement non vivant qui, par leur interaction, forment une unité fonctionnelle $\gg^{17}$. Autrement dit, il s'agirait de porter atteinte à un de ces éléments et partant, par exemple, à une plante composant un écosystème considéré. Le préjudice écologique serait ensuite une atteinte aux fonctions des écosystèmes, c'est-à-dire aux manifestations fonctionnelles des écosystèmes. Le préjudice écologique pourrait enfin être établi en cas d'atteinte aux bénéfices collectifs tirés par l'homme de l'environnement, c'est-à-dire des services écosystémiques ou encore des manifestations fonctionnelles des écosystèmes utiles à l'homme. Reprenons alors la distinction entre le dommage et le préjudice soutenue par une partie de la doctrine. Le dommage désigne le seuil de la lésion, c'est-à-dire le bien atteint, tandis que le préjudice vise la détermination des conséquences de la lésion, c'est-à-dire, les utilités perdues du bien du fait de l'atteinte ${ }^{18}$. Partant, il est possible d'affirmer que l'atteinte aux éléments des

17. $C f$. glossaire Ecosystème(s).

18. La théorie moderne de la propriété (théorisée par les professeurs Zénati-Castaing F. et Revet Th., Les biens, PUF, $3^{\mathrm{e}}$ ed., 2008) la définit de deux manières. D'une part, elle est objectivement définie en ce sens qu'il s'agit de la qualité qu'a un bien d'appartenir à une personne. Cette conception invite à revenir sur la notion de bien. Pour la doctrine de la propriété renouvelée, une chose ne devient un bien, sous réserve de la Loi, que parce qu'il est utile de se l'approprier. D'autre part, elle est subjectivement définie comme le rapport exclusif entre un sujet de droit et un bien dont le rapport permet à son titulaire de retirer toutes les utilités que le bien permet. Aussi, si l'on veut bien admettre que la propriété, structure de tous les droits subjectifs, est protégée par la responsabilité civile (Cf. Jean S., La protection des droits subjectifs par la responsabilité civile, Thèse, Toulouse, 2012) en sus de l'exercice d'actions spécifiques, alors on peut retrouver une concordance parfaite entre d'une part, le dommage et le bien, objet du droit subjectif, et d'autre part, le préjudice et l'utilité. Ainsi, le dommage permet de désigner en responsabilité le bien atteint tandis 
écosystèmes ne permet que d'identifier le bien atteint, le dommage, à partir du fait dommageable, l'atteinte aux éléments des écosystèmes. A contrario, l'atteinte aux fonctions ou aux bénéfices collectifs tirés par l'homme des écosystèmes tend à mettre en lumière non plus le dommage mais les préjudices consécutifs à l'atteinte aux éléments des écosystèmes, c'est-à-dire aux fonctions ou utilités perdues, ou du moins dégradées, des écosystèmes. Il ressort de cette analyse que le législateur, à travers l'article 1247 du Code civil, opère une confusion regrettable entre le dommage et le préjudice ; le dommage ne conduisant pas forcément à un préjudice, l'atteinte aux éléments des écosystèmes n'ayant pas pour conséquence inéluctable la dégradation ou la perte d'une fonction des écosystèmes ou d'un service écosystémique. En d'autres termes, s'il est fondamental en responsabilité civile de mettre en exergue le bien atteint, les écosystèmes donc, il est inutile, au titre des conditions de mise en œuvre, de lister les préjudices réparables pour la simple raison que le propre de la responsabilité civile, une fois engagée, a pour objet la réparation de tous les préjudices comme, par exemple, la dégradation ou la perte de fonctions des écosystèmes ou des bénéfices collectifs tirés par l'homme desdits écosystèmes.

En revanche, le législateur, en mettant sur le même plan les atteintes aux fonctions des écosystèmes et les atteintes aux bénéfices collectifs tirés par l'homme, a au moins le mérite de clarifier la distinction entre ces deux préjudices a priori réparables. Madame Fèvre, dans sa thèse, distingue clairement les deux puisque selon elle, « une fonction ne prend la forme d'un service qu'à partir du moment où les pratiques et besoins sociaux reconnaissent ce service comme tel, c'est-à-dire dès lors que la fonction est utile à la satisfaction d'un besoin humain, plus généralement, à l'accomplissement de son bien-être, et non à une entité naturelle $»^{19}$. Autrement dit, il s'agit de dire que les écosystèmes ont à la fois des utilités intrinsèques - les fonctions des écosystèmes - et des utilités extrinsèques - les bénéfices collectifs tirés par l'homme - parce qu'elles profitent à l'homme et qu'elles sont perçues ainsi par l'homme. Pour le dire encore différemment, un écosystème dispose de fonctions propres qui profitent à d'autres écosystèmes (utilités intrinsèques) ou à

que le préjudice autorise à identifier les utilités dégradées ou perdues du bien en raison de l'atteinte.

19. Fèvre M., op. cit., p. 49. 
1'homme (utilités extrinsèques). Cette distinction fait écho à la proposition faite par le professeur Guillaume Beaussonie de voir dans les services écosystèmiques des utilités extrinsèques d'un bien - les écosystèmes - « dont la particularité est de bénéficier à tous, bien au-delà de leur seul propriétaire, qui ne saurait empêcher son déploiement $»^{20}$. Aussi, c'est sans doute en raison de cette particularité - utilité bénéficiant à tous - qu'il est difficile pour la responsabilité civile de se saisir de ce préjudice réparable dans la mesure où cette dernière est construite sur l'idée que le préjudice réparable doit être personnel alors que les services écosystémiques constituent des bénéfices collectifs.

Si la dégradation ou la perte de fonctions des écosystèmes, en tant que préjudices réparables, se heurte assurément à l'exigence du caractère personnel en matière de responsabilité civile sauf à considérer, comme le professeur Mireille Bacache, que l'adaptation de la responsabilité civile à l'évolution des besoins indemnitaires doit conduire à se contenter d'exiger un préjudice objectif ${ }^{21}$, la problématique est la même lorsqu'il s'agit de s'intéresser à la dégradation ou la perte des bénéfices collectifs tirés par l'homme de l'environnement. En effet, comme l'indique le professeur Mustapha Mekki, le préjudice écologique peut être concret ou subjectif qu'il soit collectif ou individuel ${ }^{22}$. Si l'on imagine assez bien qu'une victime puisse, à titre individuel, solliciter, sur le fondement de l'un des faits générateurs de responsabilité civile du Code civil ${ }^{23}$ (faits personnels, faits des choses ou faits d'autrui), l'indemnisation de son préjudice personnel à la condition de démontrer qu'il a perdu un service écosystémique, c'est-à-dire une utilité de son bien qui peut être collective mais qui doit être assurément individuelle, en revanche, les choses se compliquent lorsqu'il est question d'obtenir la réparation de la dégradation ou de la perte des services écosystémiques visés par l'article 1247 du Code civil. En qualifiant les bénéfices de l'environnement comme des bénéfices collectifs, on retombe inexorablement en opposition avec la conception classique

20. Beaussonie G., « La qualification juridique des services écosystémiques ».

21. Bacache M., «L'action de groupe en matière environnementale », EEI n 3, Mars 2017, étude $8, \mathrm{n}^{\circ} 24$ et ss.

22. Mekki M., «Responsabilité civile et droit de l'environnement. - Vers un droit spécial de la responsabilité civile environnementale ? ", Responsabilité civile et assurances, $\mathrm{n}^{\circ} 5$, Mai 2017, dossier 4, n 17.

23. A l'exception des articles 1246 à 1252 du Code civil. 
de la responsabilité civile exigeant le caractère personnel du préjudice. Pourtant, les articles 1246 et suivants du Code civil n'ont aucunement vocation à réparer des préjudices individuels. Il suffit pour s'en convaincre de lister les personnes habilitées à agir - l'Etat ou par exemple l'Agence française pour la biodiversité - ou encore le régime de réparation avec notamment l'affectation possible des dommages et intérêts. Aussi, de deux choses l'une : soit la réparation du préjudice écologique au titre duquel figurent les services écosystémiques conduisent effectivement à la déformation ou, à tout le moins, à l'adaptation de la responsabilité civile des articles 1246 et suivants du Code civil ; soit il ne s'agit pas, à proprement parler, de responsabilité civile. En tout état de cause, les services écosystémiques offrent l'opportunité de repenser non plus seulement les conditions de la responsabilité civile mais la responsabilité civile elle-même.

\section{B. Les SERVICES ÉCOSYSTÉMiQUeS AU DÉTRIMENT DE LA RESPONSABILITÉ CIVILE ELLE-MÊME}

Celles et ceux qui s'intéressent à la responsabilité civile savent qu'elle a toujours su s'adapter aux nécessités indemnitaires de son temps. Il y a finalement deux manières, parfois cumulatives d'ailleurs, de réussir ce tour de force. Soit en jouant sur le régime de responsabilité civile en facilitant, par exemple, l'indemnisation du préjudice corporel ; soit en s'attaquant aux conditions de mise en œuvre de la responsabilité civile. Dans cette dernière hypothèse, il est alors possible, par exemple, de choisir un fait générateur plus facile à rapporter, de reconnaître de nouveaux préjudices réparables ou encore de diminuer l'exigence relative à l'établissement du lien de causalité. Aussi, si l'on veut bien admettre que la responsabilité civile, lorsqu'elle poursuit comme finalité l'indemnisation des préjudices qu'ils soient individuels ou collectifs, demeure de la responsabilité civile alors il importe que ces conditions de mise en œuvre s'adaptent à l'objectif visé, à la condition toutefois que ces conditions demeurent. Dès lors, rien n'interdit le rattachement du préjudice écologique, en l'occurrence la dégradation ou la perte des bénéfices collectifs tirés par l'homme, à la responsabilité civile quand bien même serait-elle une responsabilité civile spéciale comme l'a voulu le législateur en lui dédiant un chapitre spécifique au sein du Code civil. Par conséquent, les services écosystémiques ne sont tant intéressants pour rejeter leur rattachement à la responsabilité civile que pour repenser la responsabilité civile elle-même. 
La responsabilité civile a toujours été pensée à partir du fait générateur. Ainsi, le Code civil recense des régimes de responsabilité civile reposant sur des faits personnels, des faits des choses ou encore des faits d'autrui. Pourtant, une partie de la doctrine milite, depuis des décennies, pour la construction de régimes de responsabilité civile fondés non plus sur un fait générateur mais en fonction de la nature du dommage ${ }^{24}$. Le droit positif le fait en réalité déjà au titre de certains régimes de responsabilité civile puisque, par exemple, la victime d'un accident de la circulation obtiendra assurément l'indemnisation des préjudices consécutifs à un dommage corporel quand bien même elle aurait commis une faute - du moment qu'elle n'est pas inexcusable et cause exclusive de l'accident - alors que la même victime verra son indemnisation limitée ou exclue pour les autres dommages ${ }^{25}$. La détermination d'une ratio légis adaptée à la nature du dommage va dans le sens de l'histoire de la responsabilité civile. D'ailleurs, le projet de réforme de la responsabilité civile de la Chancellerie va dans cette direction dans la mesure où, par exemple, des règles spécifiques sont prévues selon que les préjudices résultent d'un dommage matériel ou corpore ${ }^{26}$. Cela étant, la prise en compte de la nature du dommage n'est pour l'heure envisagée qu'au stade des régimes de certaines responsabilités civiles et non pas à celui des conditions de mise en œuvre. Pourtant, il apparait possible, avec l'insertion de la réparation des services écosystémiques, de concevoir une responsabilité civile fondée exclusivement à partir du dommage.

L'idée n'est pas nouvelle puisque Boris Starck, dans sa thèse consacrée à la théorie de la garantie, proposait déjà une hiérarchisation des intérêts protégés - à l'allemande - par le truchement de la nature des dommages ${ }^{27}$. Depuis, une partie de la doctrine estime que l'atteinte au corps - le dommage corporel donc - est l'intérêt protégé supérieur de telle manière qu'il justifierait un traitement spécifique au profit des victimes. Les autres, en revanche, parce qu'ils constituent des intérêts de moindre importance exigeraient, à la fois ou soit, des

24. Starck B., Essai d'une théorie générale de la responsabilité civile considérée en sa double fonction de garantie et de peine privée, thèse, éd. Rodstein, 1947.

25. Art. 3 et 5 de la loi n ${ }^{\circ} 85-677$ du 5 juillet 1985 tendant à l'amélioration de la situation des victimes d'accidents de la circulation et à l'accélération des procédures d'indemnisation.

26. $C f$. les articles 1267 et suivants du projet de réforme de la Chancellerie présenté le 13 mars 2017.

27. Starck B., op. cit. 
conditions de mise de la responsabilité civile davantage drastiques - une faute par exemple - ou un régime de responsabilité civile moins bienveillant comme, par exemple, l'exigence d'une valeur minimale du dommage lorsque ce dernier est matériel ${ }^{28}$. Ce qui est frappant en matière de services écosystémiques quand il s'agit d'évoquer la responsabilité civile instituée aux articles 1246 et suivants du Code civil, c'est l'absence d'exigence d'un fait générateur spécifique. Autrement dit, la nature du fait générateur n'importe pas puisqu'il suffit qu'un dommage écologique survienne, c'est-à-dire l'atteinte à un écosystème, pour mobilier cette responsabilité civile.

En revanche, la rédaction de l'article 1247 du Code civil renseigne sur la place qu'a voulu lui accorder le législateur au titre de la hiérarchisation des intérêts protégés. En effet, cette disposition exige une atteinte «non négligeable » aux éléments des écosystèmes. En d'autres termes, il ne suffit pas qu'un dommage écologique survienne mais un dommage écologique d'une certaine gravité. Si la gravité du dommage n'est pas inconnue en responsabilité civile puisqu'elle permet de différencier le traitement des préjudices au stade de certains régimes de responsabilité - en matière de produits défectueux par exemple - elle n'était, jusqu'à la consécration de la réparation du préjudice écologique dans le Code civil, aucunement requise au titre des conditions de mise en œuvre des régimes de responsabilité civile. Cette nouveauté conduit à affirmer que l'environnement n'est pas - du moins pour la responsabilité civile - l'intérêt protégé le plus important. L'exigence d'une gravité du dommage au stade des conditions de mise en œuvre de la responsabilité civile est condamnable à au moins deux égards. D'une part, comme le suggère Monsieur Huglo, un «petit » dommage peut conduire à de " grands » préjudices tandis qu'un " grand » dommage peut donner lieu à de «petits » préjudices $^{29}$. D'autre part, la gravité du dommage écologique tend à créer une inégalité de traitement - toujours au stade des conditions de mise en ouvre de la responsabilité civile - puisque la responsabilité civile des articles 1246 et suivants du Code civil ne permet la réparation notamment que de la dégradation ou de la perte des bénéfices collectifs tirés par l'homme de l'environnement lorsque ces préjudices ne sont pas

28. C'est déjà le cas en matière de produits défectueux où le dommage doit avoir une valeur supérieure à 500 euros.

29. Huglo Ch., « La difficile application de la réparation du préjudice écologique devant le juge », EEI, n 6 , Juin 2017, dossier 15, n 3 . 
individuels. Or, on peut parfaitement imaginer qu'une victime, pour ces mêmes préjudices, en sollicite l'indemnisation parce qu'ils lui sont personnels et individuels. Si tel est le cas, certes la victime devra se fonder sur un autre régime de responsabilité civile du Code civil, mais la gravité du dommage écologique ne sera pas exigée au stade des conditions de mise en œuvre de la responsabilité civile choisie. Dès lors, cela ne signifie pas que l'intérêt protégé - l'écosystème ait fait l'objet d'une hiérarchisation tandis que cela conduit à une inégalité de traitement des victimes dans la mesure où les bénéfices collectifs titrés par l'homme peuvent être à la fois des utilités extrinsèques collectives et des utilités extrinsèques individuelles. En définitive, les services écosystémiques ont assurément une incidence sur les conditions de mise en œuvre de la responsabilité civile. Reste à savoir si tel est également le cas lorsqu'on s'intéresse au régime de la responsabilité civile.

\section{L'INCIDENCE DES SERVICES ÉCOSYSTÉMIQUES SUR LE RÉGIME DE RESPONSABILITÉ CIVILE}

Les services écosystémiques, par le truchement du dommage écologique, ont également une incidence quant au régime de responsabilité civile à au moins deux niveaux. D'une part, lorsqu'il s'agit de s'intéresser aux aspects procéduraux de l'action qui certes ne sont pas rédhibitoires pour l'efficacité de l'action mais qui doivent faire l'objet d'éclaircissement afin d'ailleurs de pouvoir disposer d'un socle cohérent pour prendre en charge le dommage écologique (A). D'autre part, le même constat, avec cette fois-ci beaucoup plus d'inconvénients, doit être dressé quant à la réparation elle-même dont le régime est pour l'heure insatisfaisant ou, tout du moins, peu enclin à donner envie d'agir (B).

\section{A. Les services écosystémiques au stade de L'action en Respon- SABILITÉ CIVILE}

L'article 1248 du Code civil prévoit que « l'action en réparation du préjudice écologique est ouverte à toute personne ayant qualité et intérêt à agir, telle que l'Etat, l'Agence française pour la biodiversité, les collectivités territoriales et leurs groupements dont le territoire est concerné, ainsi que les établissements publics et les associations agréées ou créées depuis au moins cinq ans à la date d'introduction de l'instance qui ont pour objet la protection de la nature et la 
défense de l'environnement ». Cette disposition, qui doit notamment permettre l'indemnisation de la dégradation ou de la perte de services écosystémiques, est l'occasion de mettre en évidence les aspects procéduraux de l'action en responsabilité civile ${ }^{30}$. En effet, à travers l'exigence de la qualité et de l'intérêt agir, c'est la question du cumul des actions qui doit retenir notre attention.

Par principe, le cumul de ces deux conditions procédurales n'est pas exigé dans la mesure où, conformément à l'article 31 du Code de procédure civile, l'intérêt à agir une fois acquis, il n'est pas nécessaire de rechercher une qualité à agir. Ce n'est que par exception, lorsque l'action est dite « attitrée », que le législateur offre, à des personnes déterminées dépourvues d'intérêt personnel à agir, la qualité à agir pour défendre un intérêt. Or, l'article 1248 du Code précité ne procède aucunement de la sorte puisque qualité et intérêt agir sont requis. L'on ne peut qu'espérer qu'il s'agisse d'une maladresse dans la rédaction de la disposition car on voit mal pourquoi le législateur aurait dressé une liste ${ }^{31}$ des personnes ayant la qualité à agir pour exiger d'elles qu'elles démontrent en sus un intérêt à agir. Il est donc plus logique de penser que législateur a voulu procéder à une dissociation. D'une part, les personnes visées en disposant de la qualité à agir n'ont pas à démontrer l'intérêt à agir ; d'autre part, toutes les autres personnes doivent quant à elles démontrer un intérêt à agir duquel sera déduite la qualité à agir. Pour autant, le recours régulier à une telle action en réparation de préjudices collectifs, sans y adjoindre une action en réparation des préjudices personnels, semble peu probable sauf à considérer que l'altruisme suffira à combattre l'inertie de celles et ceux qui n'ont rien de personnel à y gagner. Aussi, c'est davantage sur un cumul d'actions qu'il faut compter pour tendre notamment à l'indemnisation de la dégradation ou de la perte d'un service écosystémique.

Si l'on met de côté la responsabilité environnementale, qui fait office d'une police administrative peu performante, plusieurs actions civiles peuvent se cumuler puisqu'elles ne poursuivent pas les mêmes fins quand bien même il s'agirait d'obtenir la réparation des préjudices consécutifs à un dommage environnemental. En effet, plusieurs

30. Il n'est pas question ici de le développer dans cette contribution mais il est à noter également un risque de confusion entre la recevabilité et le bien-fondé de l'action. En ce sens, cf. Mekki M., op. cit., ${ }^{\circ} 11$.

31. A priori non exhaustive par l'utilisation de la locution « tel que ». 
intérêts peuvent être lésés pour un même dommage corporel : ceux des personnes qui subissent personnellement un préjudice et qui devront alors agir, soit à titre individuel en recourant à l'une des responsabilités civiles prévues par le Code civil à l'exception de celle consacrée aux articles 1246 à 1252 du Code civil, soit par le truchement de l'action de groupe de l'article L. 142-3-1 du Code de l'environnement; ceux des personnes qui subissent un préjudice collectif et qui devront alors intenter leur action sur le fondement des articles 1246 et suivants du Code civil. C'est à ce stade qu'il est indispensable de procéder à la qualification des préjudices et pour ce faire, pourquoi pas, recourir à une nomenclature des préjudices environnementaux ${ }^{32}$, afin de bien délimiter les actions possibles. Une fois cette identification réalisée, rien n'interdit le cumul des actions du moins lorsqu'il s'agit de distinguer entre les préjudices collectifs et les préjudices individuels; la réparation des premiers devant être recherchée sur le fondement des articles 1246 à 1252 du Code civil, les seconds exigeant de recourir soit à une action individuelle fondée sur un fait générateur du Code civil - à l'exception des articles 1246 et suivants précités - soit à une action de groupe portée sur le fondement de 1'article 142-3-1 du Code de l'environnement. En revanche, une attention toute particulière doit être portée dans cette dernière hypothèse. Le cumul est possible car parfaitement organisé. En effet, les décisions rendues sur le fondement de l'action de groupe disposent de l'autorité de la chose jugée mais seulement à l'égard des membres du groupe de telle manière qu'elle n'interdit à celles et ceux qui prétendent avoir subi un préjudice personnel d'agir à titre individuel. Plus encore, les membres du groupe ne peuvent se voir opposer l'autorité de la chose jugée de telle sorte qu'ils peuvent encore individuellement agir pour les préjudices personnels qui n'auraient pas été invoqués dans le cadre de la décision fondée sur l'action de groupe. Dès lors, sous réserve d'une délimitation des préjudices réparables et d'une interprétation conforme à la distinction entre l'intérêt et la qualité à agir, le droit s'est doté d'un arsenal juridique opérant pour prendre en charge le dommage environnemental et partant, la dégradation ou la perte des bénéfices collectifs tirés par l'homme de l'environnement. Cela étant, l'étude, au titre du régime de cette responsabilité civile, montre

32. Neyret L. et Martin G.-J., Nomenclature des préjudices environnementaux, LGDJ, 2012. 
que l'enthousiasme doit être relatif lorsqu'il s'agit de s'intéresser à la réparation des préjudices.

\section{B. LES SERVICES ÉCOSYSTÉMIQUES AU STADE DE LA RÉPARATION}

Les atteintes aux bénéfices collectifs tirés par l'homme de l'environnement une fois identifiées et mesurées, le Code civil a fait le choix de hiérarchiser les modes de réparation en privilégiant, au titre de l'article 1249 alinéa 1 du Code civil ${ }^{33}$, la réparation en nature qui est la forme la plus conforme à l'idée de réparation puisqu'il s'agit de supprimer le préjudice. Aussi, ce n'est qu'à défaut, c'est-à-dire en cas d'impossibilité ou d'insuffisance de la réparation en nature, qu'il est possible de recourir à l'allocation de dommages et intérêts. La réparation en nature n'appelle pas d'observations particulières car elle a le mérite d'être en adéquation avec le préjudice subi. Autrement dit, si la réparation en nature consiste à rétablir le service écosystémique perdu, alors cette responsabilité joue pleinement son rôle.

En revanche, lorsque la réparation se réalise par le truchement de dommages et intérêts, il n'est pas certain que la responsabilité civile permette le rétablissement de la situation de la victime quand bien même, en faisant une entorse au principe de non-affectation des dommages et intérêts ${ }^{34}$, elle affecterait ces fonds à la réparation de l'environnement. Au-delà de la violation du principe de nonaffectation, l'affectation des dommages et intérêts, telle que prévue par le législateur risque de ne pas atteindre l'objectif recherché puisqu'il est à craindre une inadéquation entre le préjudice subi et l'affectation retenue. En effet, l'article 1249 alinéa 2 du Code civil dispose qu' « en cas d'impossibilité ou de droit ou de fait ou d'insuffisance des mesures de réparation, le juge condamne

33. Art. 1249, alinéa 1 du Code civil : « La réparation du préjudice écologique s'effectue par priorité en nature ».

34. Cass. crim., 22 fév. 1995, Bull. crim. 1995, n 77. Le juge excéderait ses pouvoirs s'il décidait de l'affectation des dommages et intérêts alloués à la victime. La solution procède non seulement de la fonction des dommages et intérêts car ces derniers ont pour objectif de fournir à la victime les moyens de se procurer des satisfactions de remplacement, mais aussi du principe de libre gestion par chacun de son patrimoine : Cass. $2^{\text {ème }}$ civ., 8 juil. 2004, Bull. civ. 2004, II, $\mathrm{n}^{\circ} 391$ : « le principe de la réparation intégrale n'implique pas de contrôle sur l'utilisation des fonds alloués à la victime qui conserve leur libre utilisation ». Le projet de réforme de la responsabilité civile de la Chancellerie va également dans ce sens puisque l'article 1264 dispose que « la victime est libre de disposer des sommes allouées ». 
le responsable à verser des dommages et intérêts, affectés à la réparation de l'environnement, au demandeur ou, si celui-ci ne peut prendre les mesures utiles à cette fin, à l'Etat». Or, comme le souligne à juste titre la professeure Mathilde Hautereau-Boutonnet, l'affectation à une notion si large qu'est «l'environnement » peut conduire à ce que " les dommages-intérêts soient utilisés pour, non pas réparer le préjudice écologique constaté au litige, mais l'environnement plus généralement, au-delà de ce préjudice ${ }^{35}$. Le risque est d'autant plus probable en cas de pluralité de demandeurs à l'action. Dans cette hypothèse, il est certain que la somme allouée au titre de la réparation du même préjudice écologique, une fois divisée entre les différents demandeurs, pourra être affectée non pas nécessairement à la réparation du préjudice écologique subi mais à la réparation de l'environnement de sorte qu'il n'est pas impossible, au final, de considérer que c'est davantage le préjudice collectif qu'écologique qui est réparé. Le risque d'une inadéquation entre l'affectation des dommages et intérêts et le préjudice subi est-il réel ? Assurément quand le recours aux dommages et intérêts est conditionné par l'impossibilité de procéder à la réparation en nature ou par l'insuffisance de cette dernière ! Les juges peuvent d'abord se heurter à une impossibilité de fait en raison de la complexité des mesures à mettre en œuvre pour tenter de réparer le préjudice subi ; les juges peuvent ensuite retenir une impossibilité de droit tenant, par exemple, à la séparation des autorités judiciaires et administratives lorsque les mesures envisagées contredisent sévèrement une autorisation administrative qui aurait été délivrée ; les juges disposent enfin d'un pouvoir très étendu dans la mesure où le seul constat de l'insuffisance des mesures prises offre la possibilité d'allouer des dommages et intérêts. Dès lors, il est regrettable que le législateur ne se soit pas donné les moyens de s'assurer que l'affectation des dommages et intérêts soit en conformité avec le préjudice écologique subi car à la complexité de l'appréhension de notions, comme celle de services écosystémiques au stade des conditions de mise en œuvre de la responsabilité civile, il a ajouté la complexité d'un régime difficilement praticable ${ }^{36}$. Plus encore, le recours aux dommages et intérêts en cas de pluralité de demandeurs pour une même action en responsabilité civile fondée sur les articles

35. Hautereau-Boutonnet M., «Quelle action en responsabilité civile pour la réparation du préjudice écologique ? ", EEI, $\mathrm{n}^{\circ}$ 6, Juin 2017, dossier 14, n 20.

36. Pour quelques solutions pour faire face à ces difficultés, cf. Hautereau-Boutonnet M., ibid., $\mathrm{n}^{\circ} 23$. 
1246 et suivants du Code civil, couplé avec d'autres actions en responsabilité civile recherchant cette fois-ci l'indemnisation de préjudices personnels - par le truchement de l'action de groupe de l'article L. 142-3 du Code de l'environnement ou trouvant un fondement dans un fait personnel, un fait des choses ou d'autrui du Code civil - peut conduire à un imbroglio juridique. Au-delà de la difficulté à identifier les chefs de préjudices réparables, c'est l'affectation démultipliée des dommages et intérêts aux différentes victimes qui risque de se heurter au principe de la réparation intégrale si les sommes allouées dépassent le(s) préjudice(s) réparable(s). Or, pour l'heure du moins ${ }^{37}$, les dommages et intérêts punitifs ne sont pas admis et quand bien même ils le seraient, il conviendrait, au moins pour les actions fondées sur les articles 1246 et suivants du Code civil, qu'ils se justifient non pas eu égard à la gravité du dommage mais eu égard à la gravité du fait générateur nécessairement fautif.

Il ressort de cette analyse que l'intégration des services écosystémiques, par le truchement du préjudice écologique, au sein de la responsabilité civile influence assurément cette dernière soit parce qu'elle oblige à l'adapter, voire la déformer, soit parce qu'elle invite à la repenser. Si pour l'heure cette responsabilité civile semble insuffisamment précisée tant au niveau de ses conditions de mise en œuvre qu'au niveau de son régime pour être efficiente, il faut reconnaître qu'elle ouvre ou ré(ouvre) des réflexions pour matricer une responsabilité civile adaptée aux défis du XXI ${ }^{\text {ème }}$ siècle au titre desquels figurent prioritairement le maintien du financement de la politique d'indemnisation des préjudices subis et la préservation d'un intérêt bien supérieur encore au préjudice corporel : l'environnement. Pour ce faire, de nombreuses voies sont possibles en dehors mais aussi au-dedans de la responsabilité civile. Il est rassurant de voir que la responsabilité civile s'adapte, créé de nouveaux outils comme ici en consacrant - à l'occasion du préjudice écologique - une véritable action substantielle et autonome en cessation de l'illicite ${ }^{38}$, prospecte en imaginant les outils de demain à l'image de la reconnaissance des dommages et intérêts punitifs.

37. Le projet de réforme de la responsabilité civile de la Chancellerie présenté le 13 mars 2017 le prévoit à l'article 1266-1.

38. L'article 1252 du Code civil dispose qu' "indépendamment de la réparation du préjudice écologique, le juge, saisi d'une demande en ce sens par une personne mentionnée à l'article 1248, peut prescrire les mesures raisonnables propres à prévenir ou faire cesser le dommage ». 
A N N E X E S 



\section{GLOSSAIRE GÉNÉRAL}

Les définitions à suivre correspondent à celles qui sont le plus largement diffusées dans les publications internationales, qu'il s'agisse de rapports institutionnels ou de travaux de recherche. Elles ne font néanmoins pas toujours l'objet d'un consensus et nous avons alors choisi de faire apparaître certains points en discussion sans résoudre le débat.

\section{Agriculture de conservation des sols (ACS)}

La Food and Agriculture Organisation ${ }^{1}$ des Nations Unies pose la définition formelle de l'agriculture de conservation autour de trois principes indissociables, à savoir :

- une rotation culturale allongée et diversifiée

- un couvert organique permanent des sols

- une perturbation minimale du sol

L'allongement et la diversification de la rotation culturale est le premier levier de toute démarche agroécologique. Une rotation complexe et bien gérée permet de prévenir les problèmes de compaction du sol, de maladies et d'adventices pérennes. Elle joue également un rôle important dans le maintien de la fertilité tout en limitant le risque ravageur. A cette rotation diversifiée, l'agriculture de conservation associe l'implantation de couverts végétaux en interculture ainsi qu'un maintien des résidus de culture in situ. Le sol est ainsi couvert en permanence par une couverture organique vivante ou morte (mulch) qui le protège de la destruction mécanique par la pluie, agit comme une barrière contre le vent et tamponne la température à la surface du sol. Ces couverts sont également au cœur de la gestion de la fertilisation notamment grâce à l'inclusion d'espèces de Fabacées (qui fixent par voie symbiotique l'azote atmosphérique). Enfin, l'agriculture de conservation repose sur 
une perturbation minimale du sol. L'enjeu est particulièrement de protéger la zone de surface, entre 0 et $20 \mathrm{~cm}$ de profondeur, qui est la plus biologiquement active mais également la plus vulnérable à 1'érosion. Beaucoup de fonctions biologiques et services écosystémiques essentiels sont dépendants de la vie dans cette zone.

La définition pratique de l'agriculture de conservation est encore sujette à discussion et aucune définition légale ou réglementaire de l'agriculture de conservation n'existe à ce jour.

\section{Agroécologie}

Le terme a été introduit pour la première fois dans une publication scientifique en 1928 par B.M Bensin ${ }^{2}$, un agronome Russe en poste aux USA. Il s'agissait de qualifier l'utilisation de concepts d'écologie pour la recherche agronomique. Les préoccupations à l'origine de son développement sont alors la lutte contre les problèmes d'érosion et de pollutions diffuses.

On distingue communément trois dimensions de l'agroécologie : c'est à la fois une discipline scientifique, un mouvement social et un ensemble de pratiques agricoles ${ }^{3}$.

Seule la dernière dimension est intégrée par le droit, c'est-à-dire l'ensemble des pratiques constituant les systèmes de production dits agroécologiques. La définition de l'agroécologie est donnée par l'article $1^{\text {er }}$ de la loi d'avenir pour l'agriculture, l'alimentation et la forêt du 13 octobre 2014 : les « systèmes de production agroécologiques, dont le mode de production biologique,... combinent performance économique, sociale, notamment à travers un haut niveau de protection sociale, environnementale et sanitaire ». Plus concrètement, « ces systèmes privilégient l'autonomie des exploitations agricoles et l'amélioration de leur compétitivité, en maintenant ou en augmentant la rentabilité économique, en améliorant la valeur ajoutée des productions et en réduisant la consommation d'énergie, d'eau, d'engrais, de produits phytopharmaceutiques et de médicaments vétérinaires, en particulier les antibiotiques. Ils sont

2. Bensin, B.M, 1928, Agroecological characteristics description and classification of the local corn varieties chorotypes, Editeur inconnu.

3. Wezel A, Bellon S, Doré T, et al., 2009, Agroecology as a science, a movement and a practice. A review. Agronomy for Sustainaible Development, 29, 503-515. 
fondés sur les interactions biologiques et l'utilisation des services écosystémiques et des potentiels offerts par les ressources naturelles, en particulier les ressources en eau, la biodiversité, la photosynthèse, les sols et l'air, en maintenant leur capacité de renouvellement du point de vue qualitatif et quantitatif. Ils contribuent à l'atténuation et à l'adaptation aux effets du changement climatique $»$.

Le développement de ces systèmes agroécologiques est soutenu par l'agroécologie en tant que discipline scientifique. Elle a pour objet la modernisation de l'agriculture avec un objectif de performance économique, environnementale et sociale et se doit d'avoir un regard critique envers toutes les agricultures, de la plus intensive à la plus extensive. Au-delà d'une simple « science de l'application des concepts écologiques pour la mise en place de systèmes de production durables $»^{4}$, l'agroécologie est transdisciplinaire, intégrant l'agronomie, l'écologie, la sociologie et l'économie pour l'étude des interactions entre plantes, animaux, humains et l'environnement aux différentes échelles des systèmes de production alimentaire, « de la graine à la table».

L'agroécologie est également reconnue comme étant un mouvement social voire politique. Initiée dans les années 60 , suite à la révolution verte et à la prise de conscience grandissante des impacts négatifs de l'agriculture industrielle sur les écosystèmes, cette approche de l'agroécologie englobe une plus large diversité d'acteurs, politiques, industriels, consommateurs... Cette dimension ne sera pas directement traitée dans cet ouvrage.

\section{Biodiversité}

Aux termes de l'arrêté fixant le vocabulaire officiel de l'environnement (JORF du 12 avril 2009, page 6438) biodiversité et diversité biologique sont synonymes. La biodiversité correspond à la « diversité des organismes vivants, qui s'apprécie en considérant la diversité des espèces, celle des gènes au sein de chaque espèce, ainsi que l'organisation et la répartition des écosystèmes ».

4. Gliessman SR, 2006, Agroecology: The Ecology of Sustainable Food Systems. New York, USA, CRC Press Taylor \& Francis, New York. 
Du point de vue des écologues, la composante fonctionnelle de la biodiversité, c'est-à-dire la diversité des fonctions écologiques, manque à cette définition. En effet, la diversité des organismes vivants n'est, à elle-seule, pas nécessairement gage d'une diversité et surtout d'une résilience des fonctions écologiques ; or ces fonctions sont à la fois indispensables au maintien de l'état de l'écosystème et le support des services écosystémiques que l'Homme retire des écosystèmes.

\section{Compensation écologique}

La compensation peut prendre différentes formes mais dans toutes les situations elle véhicule l'idée d'un rééquilibrage ; un avantage vient contrebalancer un désavantage. Cet objectif de neutralité écologique implique d'être en capacité de mesurer lesdits avantages et inconvénients puis de s'assurer que la compensation restaure en quantité et en qualité à hauteur de la détérioration, et de veiller à ce qu'elle dure aussi longtemps que les actions de dégradation. En d'autres termes, les résultats de la compensation doivent être équivalents écologiquement aux pertes induites par les impacts du projet.

\section{Disservice(s) / Dysservice(s)}

Ce terme n'existe pas dans la langue française, et certains emploient plutôt le terme de " dommage ». Malgré tout, il est de plus en plus employé en (agro)écologie. Dans cet ouvrage nous lui préfèrerons son orthographe francisée à savoir « dysservice ».

Les dysservices correspondent aux divers états ou processus liés au fonctionnement des écosystèmes qui sont, contrairement aux services écosystémiques, considérés comme négatifs pour l'homme. Dans l'exemple donné par Mark Sagoff en 2011, un producteur d'amandiers perçoit la pollinisation comme un service alors que son voisin, producteur de mandariniers auto-féconds, la conçoit comme un dysservice. En effet, le second producteur a investi dans des arbres procurant des mandarines sans pépin qui peuvent être vendues plus chères et la pollinisation favorise le retour de mandariniers produisant des fruits à pépins ${ }^{5}$. 
Cette définition reste floue ce qui la rend à la fois controversée et peu opérationnelle. D'abord, un service pour certains peut être un dysservice pour d'autres comme l'atteste l'exemple ci-dessus. Ensuite, l'avantage pour l'homme est envisagé dans le court ou le moyen terme. Or, un dysservice immédiat peut constituer un service sur le long terme, et inversement, un service immédiat s'avérer constituer un dysservice futur en conséquence d'évolutions globales de l'environnement ou des sociétés humaines. Enfin, un dysservice pour l'humain peut s'avérer être bénéfique au fonctionnement de l'écosystème. Par exemple, un incendie est susceptible de renforcer la résilience d'un écosystème.

\section{Ecosystème(s)}

Un écosystème est un complexe dynamique formé de communautés de plantes, d'animaux et de micro-organismes et de leur environnement non vivant qui, par leur interaction, forment une unité fonctionnelle.

Les limites qui définissent physiquement un écosystème sont souvent arbitraires, l'unité fonctionnelle pouvant être variable selon l'échelle de travail (de très locale à nationale voire mondiale, selon les organismes/processus étudiés) et le niveau de précision considéré.

\section{Processus et fonctions écologiques}

Les écosystèmes peuvent s'appréhender au travers d'une chaîne d'éléments imbriqués : les entités qui les composent (ex. les espèces animales, végétales et leurs races et variétés), les processus dans lesquels ces entités interviennent (ex. la reproduction des végétaux, le butinage par les abeilles) et les fonctions générées par ces processus (ex. la pollinisation).

Ces phénomènes sont fondamentaux dans l'équilibre et le fonctionnement des écosystèmes (cycle des nutriments, formation des sols, production primaire, etc.) et se mettent en place indépendamment d'un éventuel bénéficiaire humain.

La distinction formelle entre processus et fonctions écologiques est encore parfois sujette à débat (voir définitions du MEA 2005, 
du TEEB $2010^{6}$ ou du CGDD 20107), néanmoins, ces termes se réfèrent tous deux à une vision éco-centrée du fonctionnement des écosystèmes et qualifient des phénomènes propres à l'écosystème et nécessaires au maintien de son état écologique, physique et chimique, contrairement à la notion de service écosystémique, qui, anthropocentrée, fait nécessairement référence à un usage ou un bénéfice pour l'Homme.

Il n'y a pas de définition donnée par le droit à la notion de « fonctions écologiques ». A défaut d'énoncés prescriptifs, la doctrine a récemment décrit les fonctions écologiques comme des « interactions entre les éléments et les processus biologiques et physiques qui permettent le maintien et le fonctionnement des écosystèmes ». Dans le même ouvrage, il est précisé que « les fonctions écologiques des sols s'entendent du rôle qu'ils jouent au sein des écosystèmes, tel que, par exemple : servir de vivier à la biodiversité, contribuer au stockage au filtrage et à la transformation d'éléments nutritifs, de substances et d'eau, à la recharge des nappes souterraines, à la séquestration du carbone ou encore à la régulation du carbone ${ }^{8}$.

Toutefois, il faut d'ores et déjà annoncer que la distinction entre fonction écologique et service écosystémique est malaisée. En effet, la fonction écologique (qui est d'ailleurs la résultante de plusieurs processus) ne prend le statut de service que lorsque l'homme peut en tirer potentiellement un avantage. Certains auteurs critiquent l'usage abusif des termes « service écosystémique » pour qualifier des fonctions dont la finalité n'est pas le bénéfice humain. C'est notamment le cas de certains services de régulation et des services liés au fonctionnement des sols pour lesquels l'Homme doit mettre en œuvre du capital pour que la fonction écologique (par ex. la prédation des pucerons) soit valorisée comme service écosystémique (ex. régulation des ravageurs du blé, seulement si le blé est récolté).

6. TEEB, 2010, The Economics of Ecosystems Biodiversity, The Economics of Ecosystems and Biodiversity: Mainstreaming the Economics of Nature: A Synthesis of the Approach Conclusions and Recommendations of TEEB, Progress Press, Malta.

7. Projet de caractérisation des fonctions écologiques des milieux en France. Service de l'économie, de l'évaluation, et de l'intégration du développement durable, Etudes et Documents.

8. Neyret L. et Martin G.-J. (dir.), Nomenclature des prejudices environnementaux, LGDJ, 2012, p. 16. 


\section{Qualité / Santé des sols}

La santé des sols peut se définir comme la capacité d'un sol à fonctionner sur le long terme à l'instar d'un système vivant, à la fois dans les limites de cet écosystème et avec son environnement extérieur (Dictionnaire d'Agroécologie de l'INRA, 20179).

Il s'agit notamment de la capacité de l'écosystème sol à assurer la production végétale nécessaire au maintien à long terme des fonctionnalités écologiques de l'écosystème naturel ou cultivé, tout en participant à la préservation qualitative et quantitative des ressources naturelles que sont l'air, l'eau et la biodiversité.

Une partie de la communauté scientifique distingue cette notion de celle de la qualité des sols qui se focalise principalement sur les propriétés physico-chimiques des sols, pendant que d'autres considèrent les deux notions comme synonymes.

Quoi qu'il en soit, la préservation et l'amélioration de la santé des sols, de par ses composantes tant biotiques qu'abiotiques, sont désormais reconnues comme éléments clés de toute conception de systèmes agronomiques durables.

Pour l'heure, ni la qualité, ni la santé des sols ne sont définies par le droit.

\section{Service(s) écosystémique(s)}

La définition de la notion de service écosystémique fait encore aujourd'hui débat. La définition et la catégorisation proposées par le Millenium Ecosystem Assessment (MEA) en 2005 sont aujourd'hui très contestées bien que de nombreux auteurs y fassent encore référence.

Dans cet ouvrage, nous nous reposerons sur le cadre conceptuel présenté et discuté par le commissariat général au développement durable dans le cadre de l'Evaluation Française des Ecosystèmes et des Services Ecosystémiques (EFESE). Dans son rapport intermédiaire ${ }^{10}$,

\section{9. http://dicoagroecologie.fr/}

10. EFESE : Rapport intermédiaire. Thema Analyses. Décembre 2016. CGDD. Service de l'économie, de l'évaluation et de l'intégration du développement durable. 
« l'EFESE définit les biens et services écosystémiques comme des avantages socioéconomiques retirés par l'homme de son utilisation durable des fonctions écologiques des écosystèmes ». Ce document précise également qu'un service «n'est écosystémique que du fait de sa dépendance étroite au fonctionnement de l'écosystème » et que « la caractérisation d'un avantage est au cœur de la notion de service. [...] Un service écosystémique peut donc être décrit par un avantage ou par une fonction écologique. Ce n'est cependant ni l'avantage ni la fonction écologique qui caractérise à lui seul le service mais bien la mise en relation entre ces deux éléments $»$.

Par ailleurs, nous adopterons autant que possible la classification internationale commune des services écosystémiques (Common International Classification of Ecosystem Services. CICES) établie par l'Agence Européenne de l'Environnement et qui subdivise les services écosystémiques en trois catégories :

- Les services d'approvisionnement - parfois dits « de prélèvement»qui sont l'ensemble des services à l'origine de biens appropriables qui peuvent être mis en marché comme l'eau propre, le bois, les céréales, les fruits et légumes, etc.

- Les services de régulation et de maintenance qui sont des bénéfices indirects du fonctionnement des écosystèmes comme la pollinisation des cultures, le contrôle des ravageurs, la stabilisation du climat ou la protection contre les catastrophes naturelles. Il s'agit en général de services d'intérêt public qui ne génèrent pas de biens appropriables, même si ce propos doit être nuancé par l'apparition des biens meubles incorporels tels que les quotas de gaz à effet de serre.

- Les services culturels et d'innovation qui sont les bienfaits récréatifs, esthétiques, éducatifs, scientifiques et spirituels tirés du fonctionnement des écosystèmes.

Notons tout de même qu'il sera parfois fait référence à d'autres catégories, notamment les « services de support», tels que définis par le MEA (2005), qui correspondent à une catégorie de services nécessaires à la production des autres services écosystémiques et ne sont donc pas directement utilisés par l'Homme. Cette catégorie n'est plus reconnue aujourd'hui par la communauté scientifique dominante comme appartenant aux services du fait qu'ils ne peuvent être directement liés à un avantage, « les services de support [...] sont [donc] considérés comme des fonctions écologiques dans l'EFESE, et non comme des services écosystémiques ». 
De plus, dans le cas des agroécosystèmes, l'expertise scientifique collective "Agriculture et biodiversité » de l'INRA ${ }^{11}$, soutient une autre classification plus opérationnelle de ces mêmes services encore très utilisée par les agroécologues et qui distingue trois catégories de services écosystémiques : «(1) les services intrants, qui contribuent à la fourniture de ressources et au maintien des supports physicochimiques de la production agricole [...] et les services qui assurent la régulation des interactions biotiques positives ou négatives $[\ldots]$; (2) les services de production contribuant au revenu agricole : il s'agit bien sûr essentiellement de la production végétale, en considérant le niveau mais aussi la stabilité temporelle et la qualité des produits, de la production animale incluant là aussi la qualité des produits ; (3) les services produits hors revenu agricole direct, qui incluent le contrôle de la qualité des eaux, la séquestration du carbone ou la valeur esthétique des paysages notamment $»$.

Comme l'ont fait remarquer différents auteurs ${ }^{12}$, trois termes sont fréquemment utilisés tantôt comme synonymes, tantôt avec des distinctions précises, à savoir les notions de services écosystémiques, de services écologiques et de services environnementaux. Dans cet ouvrage, nous suivrons l'usage le plus commun et services écosystémiques et services écologiques seront considérés comme synonymes. En revanche, la notion de service environnemental prêtant à débat selon les disciplines et ayant notamment fait l'objet de discussions juridiques pour la distinguer de la notion de services écosystémiques, nous considérerons ces deux notions comme distinctes.

\section{Service(s) environnemental(aux)}

Selon les auteurs et les disciplines, la terminologie "service environnemental » revêt une signification variable. En agronomie, cette terminologie est régulièrement utilisée comme synonyme de « services produits hors revenu agricole direct » (comme la création d'un paysage esthétique ou l'écrêtage des crues dans des prairies inondables en amont d'une ville) tels que présentés par

11. Le Roux X., Barbault R., Baudry J., Burel F., Doussan I., Garnier E., Herzog F., Lavorel S., Lifran R., Roger Estrade J., Sarthou J.-P., Trommetter M., Agriculture et biodiversité. Valoriser les synergies, 2008, Expertise scientifique collective, rapport, INRA. 12. Lamarque P., Quétier, F. et Lavorel S., 2011, The diversity of the ecosystem services concept and its implication for their assessment and management, Comptes rendus Biologies, pp. 441-449. 
l'ESCo 2008 de l'INRA et sont donc une sous-catégorie de services écosystémiques. Pour les économistes en revanche, ces services correspondent à des externalités positives découlant des actions d'un acteur. C'est par ailleurs cette vision économique des services environnementaux via les externalités qui est reprise par la FAO dans sa définition ${ }^{13}$.

L'EFESE définit les services environnementaux comme " des services que des acteurs se rendent entre eux ou rendent à la société dans son ensemble (il est question le plus souvent d'échanges de services entre fournisseurs et bénéficiaires), et qui visent à réduire la pression exercée sur les écosystèmes ou qui améliorent leur fonctionnement».

Aucune norme juridique ne propose de définition des services environnementaux. Une partie de la doctrine les envisage comme les avantages que l'homme tire du fonctionnement des écosystèmes suite à une intervention humaine ${ }^{14}$. Cette conception du service environnemental explique aussi que, quand l'homme participe au fonctionnement de l'écosystème, il peut être rémunéré par des paiements pour services environnementaux (PSE).

\section{Sol}

Le sol constitue la couche la plus externe de la croûte terrestre, soumise à l'altération et au remaniement par l'action combinée de l'eau, de l'air, de la température et des organismes vivants. Il représente ainsi la principale interface entre les mondes minéral et organique et constitue par ailleurs le plus important habitat de la biosphère. C'est une ressource naturelle majoritaire, au même titre que l'eau et l'air, et qui plus est non renouvelable du fait de sa dynamique de formation et de régénération extrêmement lente.

Il revêt une importance tant socio-économique qu'environnementale de par ses fonctions de ressource (alimentaire, biomasse, matières premières), de support (activités humaines, unité de paysage, patrimoine culturel) ainsi que de stockage, filtration et transformation

13. FAO, 2007, La situation mondiale de l'alimentation et de l'agriculture : payer les agriculteurs pour les services environnementaux, Rome : FAO

14. Cf. Fèvre M., Les services écologiques et le droit. Une approche juridique des systèmes complexes, thèse Université Côte d'Azur, 2016, p. 27. 
de nombreuses substances (eau, carbone dont il est le principal compartiment de stockage du monde). Le sol est soumis à diverses menaces de dégradation telles que l'érosion, la perte de matières organiques, la contamination, l'imperméabilisation, le tassement, la perte de biodiversité, la salinisation, les inondations et les glissements de terrain.

Pourtant, le sol ne bénéficie pas d'une protection complète et cohérente en droit. 



\section{GLOSSAIRE DES TERMES JURIDIQUES}

Les définitions qui suivent n'ont pas de portée scientifique ; elles ont pour vocation de faciliter le dialogue interdisciplinaire et la lecture de l'ouvrage pour les non-juristes.

\section{Bien}

Toute chose susceptible d'appropriation

\section{Contrat (synonyme de convention)}

Le contrat est un accord de volontés entre deux ou plusieurs personnes destiné à créer, modifier, transmettre ou éteindre des obligations.

\section{Droit positif}

Ensemble du droit applicable au moment présent.

\section{Doctrine}

Littérature juridique.

\section{Fruits}

Biens de toutes sortes que fournissent et rapportent périodiquement les biens frugifères sans que la substance de ces biens soit entamée. Ils peuvent être civils (un loyer issus d'un fermage), industriels (la récolte d'un champ) ou naturels (des fraises des bois).

\section{Immeuble}

Bien qui par nature ne peut être déplacé 


\section{Norme}

Terme employé dans une acception générale comme équivalent de règle de droit

\section{Qualification}

Opération intellectuelle consistant à faire rentrer une chose, un acte, un fait, une situation dans une catégorie juridique préexistante d'où résulte, par rattachement, le régime juridique à appliquer.

\section{Réelles}

Qui a pour objet une chose (du latin res). Exemple peut être pris des obligations réelles environnementales qui font peser des obligations environnementales sur une chose.

\section{Régime}

Ensemble des règles applicables à une catégorie juridique donnée.

\section{Servitude :}

En droit privé, il s'agit d'une charge imposée à un immeuble bâti ou non bâti (fond servant) au profit d'un autre immeuble appartenant à un propriétaire distinct (le fond dominant), comme par exemple la servitude de passage. En droit public, la servitude administrative est une obligation grevant des propriétés privées au profit de l'intérêt général, comme par exemple, les servitudes grevant l'usage des biens dans les périmètres de captage de l'eau potable. 


\section{BIBLIOGRAPHIE SÉLECTIVE}

\section{Ouvrages, thèses}

Angel M., La nature a-t-elle un prix ? Critique de l'évaluation des biens environnementaux, Presses des Mines, Paris, 1998

Bispo A., Guellier C., Martin E., Sapijanskas J., Soubelet H. et Chenu C. (coord.), Les sols. Intégrer leur multifonctionnalité pour une gestion durable, Quae, coll. Savoir Faire, Versailles, 2016

Boutonnet M. (dir.), Le contrat et l'environnement, Etude de droit interne, international et européen, PUAM, 2014

Camproux-Duffrène M.-P. et Sohnle J. (sous la dir.), Marché et environnement, Bruylant, Bruxelles, 2014

Cans Ch. (sous la dir.) La responsabilité environnementale. Prévention, imputation, réparation, Dalloz, Coll. Thèmes et commentaires, Paris, 2009

Chabert A., Expression combinée des services écosystémiques en systèmes de production agricole conventionnels et innovants : étude des déterminants agroécologiques de gestion du sol, des intrants et du paysage, Thèse Toulouse, INPT, 2017

Chardeaux M.-A., Les choses communes, L.G.D.J., Bibliothèque de droit privé, Paris, 2006

Coriat B. (sous la dir.), Le retour des communs, La crise de l'idéologie propriétaire, Les liens Qui Libèrent, Paris, 2015.

Cornu M., Orsi F. et Rochfeld J. (sous la dir.), Dictionnaire des biens communs, Quadrige-PUF, Paris, 2017

Deffairi M., La patrimonialisation en droit de l'environnement, IRJS éd., Paris, 2015

Desrousseaux M., La protection juridique de la qualité des sols, LGDJ, bibliothèque de droit de l'urbanisme et de l'environnement, Paris, 2016

Fèvre M., Les services écologiques et le droit. Une approche juridique des systèmes complexes, thèse Université Côte d'Azur, 2016

Hautereau-Boutonnet M., Truilhé-Marengo E. (sous la dir.), Quelle(s) valeur(s) pour la biodiversité ?, mare\&martin, coll. Droit, science et environnement, Paris, 2017 
Kiss A. (dir.), Carbiener R., Doumbé-Billé S., Fromageau J., Guttinger Ph., L'Écologie et la loi : le statutjuridique de l'environnement : réflexions sur le droit de l'environnement, L'Harmattan, coll. Environnement, Paris, 1989

Langlais (sous la dir.), L'agriculture et les paiements pour services environnementaux : quels questionnements juridiques?, PUR, à paraître, 2018

Lucas M., Etude juridique de la compensation écologique, LGDJ, bibliothèque de droit de l'urbanisme et de l'environnement, Paris, 2015

Maris V., Nature à vendre. Les limites des services écosystémiques, Quae, coll. Sciences en questions, Versailles, 2014

Méral P., Pesche D. (coord.), Les services écosystémiques, Repenser les relations nature et société, Quae, coll. Nature et Société, Versailles, 2016

Monteillet V., La contractualisation du droit de l'environnement, Dalloz, nouvelle bibliothèque de thèses, Paris, 2017

Neyret L. et Martin G.-J., Nomenclature des préjudices environnementaux, LGDJ, Paris, 2012

Parance B., de Saint Victor J. (sous la dir.), Repenser les biens communs, CNRS éd., Paris, 2014

Roche P., Geijzendorffer I., Levrel H., Maris V., (coordin.) Valeurs de la biodiversité et services écosystémiques. Perspectives interdisciplinaires, Quae, collection Up date Sciences technologies, Versailles, 2016

\section{Rapports}

Bellec P., Lavarde P., Lefèbvre L. et Madignier M-L, Propositions pour un cadre national de gestion durable des sols, CGEDDCGAAER, 2015

Courtoux A. et Claveirole C., La bonne gestion des sols agricoles : un enjeu de société, Avis du Conseil économique, social et environnemental, 2015

Chevassus-Au-Louis B. et al., Approche économique de la biodiversité et des services lies aux écosystèmes - Contribution à la décision publique, Centre d'Analyse Stratégique, La documentation française, Rapports et documents, Paris, 2009

Duval L., Binet T., Dupraz P., Leplay S., Etrillard C., Pech M., Deniel E., Laustriat M., Paiements pour services environnementaux et méthodes d'évaluation économique. Enseignements pour les mesures agro-environnementales de la politique agricole 
commune. Etude réalisée pour le ministère en charge de l'agriculture. Rapport final, 2016

FAO, The state of food and agriculture. Paying farmers for environmental services, 2007

FAO and ITPS, Status of the World's Soil Resources (SWSR)Technical Summary, Rome, 2015

Fondation Nicolas Hulot pour la nature et l'homme, Droits réels au profit de la biodiversité : comment le droit peut-il contribuer à la mise en cuvre des paiements pour services environnementaux?, Rapport de la mission économie de la biodiversité, 2013

Les services écosystémiques rendus par les écosystèmes agricoles. Une contribution au programme EFESE, résumé de l'étude réalisée par l'INRA, oct. 2017

Les paiements pour Préservation des Services Ecosystémiques comme outil de conservation de la biodiversité. Cadres conceptuels et défis opérationnels pour l'action, CDC Biodiversité, Mission Économie De La Biodiversité, Les Cahiers Biodiv'2050 : comprendre, février $2014, \mathrm{n}^{\circ} 1$

Articles, contributions à des ouvrages collectifs (non cités supra)

Barnaud C., Antona M. et al., Vers une mise en débat des incertitudes associées à la notion de service écosystémique, VertigO-la revue électronique en sciences de l'environnement, vol.11, $\mathrm{n}^{\circ} 1$, mai 2011

Billet P., De la relativité de la neutralité environnementale en matière de compensation écologique, EEI, n ${ }^{\circ}$ 6, Juin 2017, Dossier 10

Billet $\mathrm{Ph}$., La « neutralité environnementale » : esquisses juridiques, in Mélanges en l'honneur de François Collart-Dutilleul, Dalloz, Paris, 2017, p. 103

Boisvert V., Les services écosystémiques : un nouveau concept ?, In : Le pouvoir de la biodiversité. Néolibéralisation de la nature dans les pays émergents, Boisvert V., Thomas F., IRD Editions, coll. Objectifs Suds, QUAE, Marseille, 2015

Camproux-Duffrène M.-P., " Le marché d'unités de biodiversité : questions de principe », RJE 2008, p. 87

Combe M., Le régime juridique de l'obligation de compensation écologique, $E E I, \mathrm{n}^{\circ} 6$, juin 2017, Dossier $\mathrm{n}^{\circ} 8$

Costanza R., d'Arge R., De Groot R., Farber S., Grasso M., Hannon B., Limburg K., Naeem S., R.O'neill, Paruelo J., Raskin R-G., Sutton P., Van den Belt M., The value of the world's ecosystem services and natural capital, Nature, 1997, p. 387 
Denizot A., L'obligation réelle environnementale ou droit réel de conservation environnementale ? Brève comparaison francochilienne de deux lois estivales ", RTD civ. 2016, p. 949

Doussan I., 2015. Compensation écologique : le droit des biens au service de la création de valeurs écologiques et après ? In : Repenser la propriété, un essai de politique écologique (GuibetLafaye C., Vanuxem S., dir.), PUAM, p. 99.

Dupont V., Lucas M., « La loi pour la reconquête de la biodiversité : vers un renforcement du régime juridique de la compensation écologique ? ", Cahiers Droit Sciences \& Technologies, 2017, $\mathrm{n}^{\circ} 7, \mathrm{p} .143$.

Etrillard C., Paiements pour services environnementaux : nouveaux instruments de politique publique environnementale, Développement durable et territoires, vol. $7, \mathrm{n}^{\circ} 1$, p. 6

Etrillard C., La compensation écologique : une opportunité pour les agriculteurs, Dr. rur. mars 2016, n 441, Etude 10

Grimonprez B., «La compensation écologique d'après la loi biodiversité », Dr. Patr., $\mathrm{n}^{\circ} 263,1^{\text {er }}$ nov. 2016

Hautereau-Boutonnet M., « La reconquête de la biodiversité par la conquête du droit civil... », JCP G 2016, 948

Hermon C., L'agroécologie en droit : état et perspective, RJE., 3-2015, p. 420

Hermon C., Plaidoyer pour une simplification du droit relatif à la protection de l'environnement dans le secteur agricole, In : Les futurs du droit de l'environnement : simplification, modernisation, régression?, Doussan I. (sous la dir.), Colloque annuel de la SFDE, 20 et 21 novembre 2014, Bruylant, Bruxelles, 2016, p. 243

Langlais A., Les paiements pour services environnementaux, une nouvelle forme d'équité environnementale pour les agriculteurs? Réflexions juridiques, Dr. rural, mai 2013, $\mathrm{n}^{\circ} 413$, Etude $\mathrm{n}^{\circ} 7$

Langlais A., L'appréhension juridique de la qualité des sols agricoles par le prisme des services écosystémiques, Dr. rural, août 2015, $n^{\circ} 435$, Etude $n^{\circ} 20$

Labous K. et Gruger H., Produire de la biodiversité : un avenir pour les agriculteurs, Dr. Env., sep. 2017, n 259, p. 291

Lucas M., Le contrat au service de la compensation écologique, EEI, 2017, Dossier $n^{\circ} 11$

Lucas M., La compensation écologique des zones humides en France : vers une intégration des services écosystémiques ?, Dr. Env., 2014, n 219, p. 19. 
Martin, G.-J., La compensation écologique : de la clandestinité honteuse à l'affichage mal assuré, RJE, 4-2016, p. 606

Martin G.-J., Les biens-environnements, une approche par les catégories juridiques, RIDE, 2015/2, p. 139

Martin G.- J., Les potentialités de l'obligation réelle environnementale, Dr. Env. oct. 2016, p. 334

Martin G.-J., Les unités de compensation dans la loi n 2016-1087 du 8 août 2016 pour la reconquête de la biodiversité, de la nature et des paysages, Droit et Ville, 2017, $\mathrm{n}^{\circ} 83$

Martin G.-J., « Pour l'introduction en droit français d'une servitude conventionnelle ou d'une obligation propter rem de protection de l'environnement », RJE 2008, p. 123

Martin G.-J., L'obligation réelle environnementale : un objet juridique non identifié ?, Annales des Loyers, avril 2017, p. 123.

Pomade A., Les paiements pour services environnementaux contribuent-ils à l'émergence d'un " gradient de juridicité »?, VertigO - la revue électronique en sciences de l'environnement, Vol. 11, $\mathrm{n}^{\circ} 1$, mai 2016

Quétier F., Quenouille B., Schwoertzig F., Gaucherand S., Lavorel S. et Thiévent P., Les enjeux de l'équivalence écologique pour la conception et le dimensionnement de mesures compensatoires d'impacts sur la biodiversité et les milieux naturels, Sciences, eaux et territoires, IRSTEA, HS 25 mars 2012.

Reboul-Maupin N., Grimonprez B., Les obligations réelles environnementales : chronique d'une naissance annoncée, D. 2016, p. 2074.

Trébulle F.G., « Le marché des unités de biodiversité, quelles perspectives ? », EEI 2017, n 6, dossier 1 .

Van Lang A., La compensation des atteintes à la biodiversité : de l'utilité technique d'un dispositif éthiquement contestable, $R D I$ 2016, p. 586

Vanuxem S. «Les services écologiques ou le renouveau de la catégorie civiliste de fruits ? ", Revue de droit de McGill, 2017, $\mathrm{n}^{\circ} 62$, p. 73. 



\title{
TABLE DES MATIÈRES
}

\author{
« SERVICES ÉCOSYSTEMIQUES ET PROTECTION DES SOLS. ANALYSES \\ JURIDIQUES ET ÉCLAIRAGES AGRONOMIQUES »
}

«Avant-propos »
par Carole HERMON p. 7

\section{PROPOS INTRODUCTIFS}

« Protection des sols et services écosystémiques.

La nécessité d'une reconsidération épistémologique » par Liliane ICHER et Bastien ALIDOR

«La protection du sol en droit» par Carole HERMON.

« Le sol agricole, une ressource indispensable négligée » par Ariane CHABERT et Jean-Pierre SARTHOU

«Propriété et protection des sols.

Réflexions civilistes sur la prise en compte de la qualité des sols » par Lionel BOSC.

\section{PROPOSITIONS POUR UNE PROTECTION DES SOLS EN DROIT}
A. L'INTÉGRATION DE LA NOTION DE SERVICE ÉCOSYSTÉMIQUE EN DROIT
« Brève histoire de l'intégration de la notion
de service écosystémique en droit»
par Isabelle DOUSSAN
«Les "services écosystémiques", une notion fonctionnelle »
par Mélodie FEVRE
« La qualification juridique des services écosystémiques » par Guillaume BEAUSSONIE.
B. LES SERVICES ÉCOSYSTÉMIQUES RENDUS PAR LES SOLS DU POINT DE VUE DE L'AGRONOMIE
«Agriculture de conservation des sols et services écosystémiques » par Ariane CHABERT et Jean-Pierre SARTHOU 
C. LA NOTION DE SERVICE ÉCOSYSTÉMIQUE PEUT-ELLE FONDER UN NOUVEAU RÉGIME DE PROTECTION?

1) Service écosystémique et financements publics

« La dépense publique en matière environnementale :

l'exemple de la protection des sols » par Liliane ICHER

2) Service écosystémique et droit des contrats

« Services écosystémiques et contrat -

Quelle obligation contractuelle environnementale?»

par Matthieu POUMAREDE

« Compensation et services écosystémiques »

par Bastien ALIDOR.

« La commande publique de compensation environnementale :

un impensé de la loi Biodiversité »»

par Hélène HOEPFFNER

« Travail du sol, services écosystémiques, et bail rural» par Didier KRAJESKI.

3) Service écosystémique et droit de la responsabilité

« L'incidence des services écosystémiques

en droit de la responsabilité civile »

par Séverin JEAN

ANNEXES

p. 299

Glossaire

p. 301

Bibliographie sélective

p. 315 

Achevé d'imprimer

GN Impressions - 31340 Villematier

Email : gnimpression@gmail.com

Dépôt légal : mars 2018 
Les auteurs qui souhaitent publier leurs travaux dans la Revue DROIT et VILLE doivent adresser leur article à l'IEJUC accompagné de leurs coordonnées personnelles (adresse postale et électronique).

L'article, précédé d'un résumé d'une dizaine de lignes en français et en langue anglaise, est transmis au moins à deux membres du Comité de lecture ou du Comité scientifique en fonction de son thème. L'auteur est ensuite informé personnellement de la décision du Comité quant à la publication de l'article. En cas d'acceptation, l'article doit être transmis par l'auteur, dans le délai précisé, au secrétariat de rédaction de la Revue (florence.jammes@ut-capitole.fr) sous forme numérique sous format word (.doc ou .docx). La rédaction peut décider des modifications de forme qui sont communiquées à l'auteur lors de la relecture de l'article avant publication définitive.

À compter de son acceptation de publication, l'auteur s'engage à ne pas publier son article dans un autre périodique sans autorisation préalable du Directeur de la Revue DROIT et VILLE.

Les auteurs recevront cinq tirés-à-part de leur article sous format papier.

SITE ET DIFFUSION EN LIGNE

Le sommaire de chaque numéro de la Revue DROIT et VILLE, mentionnant le nom des auteurs, est mis en ligne sur le site Internet de l'IEJUC : http://www.iejuc.com Vous pouvez télécharger sur ce site les bulletins d'abonnement et de commande de la Revue.

Vous trouverez également sur ce site toutes les informations relatives aux activités de l'IEJUC.

Toute correspondance relative à la Revue DROIT et VILLE (publications, abonnement, commande de Revue,...) doit être adressée à :

IEJUC - Mme Florence BAYARD-JAMMES

Manufacture des Tabacs - 21 allée de Brienne - 31000 TOULOUSE

Tél. : 0561128724 - Mail : iejuc@ut-capitole.fr

Prix de la revue au numéro : 40 euros Abonnement annuel (deux numéros) : 75 euros 


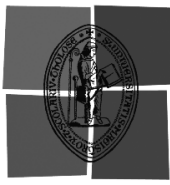
de Toulouse 